\title{
INDEXED DERMAL BIBLIOGRAPHY
}

(1995-2007) 



\section{INDEXED DERMAL BIBLIOGRAPHY (1995-2007)}




\section{This document is in the public domain and may be}

freely copied or reprinted.

\section{DISCLAIMER}

Mention of any company or product does not constitute endorsement by the National Institute for Occupational Safety and Health (NIOSH). In addition, citations to Web sites external to NIOSH do not constitute NIOSH endorsement of the sponsoring organizations or their programs or products. Furthermore, NIOSH is not responsible for the content of these Web sites. All web addresses in this document were accessible as of the publication date.

\section{ORDERING INFORMATION}

To receive NIOSH documents or other information about occupational safety and health topics, contact NIOSH at

Telephone: 1-800-CDC-INFO (1-800-232-4636)

TTY: 1-888-232-6348

E-mail: cdcinfo@cdc.gov

or visit the NIOSH Web site at www.cdc.gov/niosh

For a monthly update on news at $\mathrm{NIOSH}$, subscribe to $\mathrm{NIOSH}$ eNews by visiting www.cdc.gov/niosh/eNews

DHHS (NIOSH) Publication Number 2009-153

September 2009

SAFER ・ HEALTHIER ・PEOPLE ${ }^{T M}$ 


\section{FOREWORD}

Workers from almost every industrial sector and trade routinely experience dermal exposures to chemicals via contact with contaminated surfaces, deposition of aerosols and vapors, and immersion in or splashes from liquids. Such exposures may result in adverse health consequences ranging from direct effects to the skin (e.g., irritant contact dermatitis and corrosion) to systemic effects (e.g., cancers and neurological effects) and to sensitization (e.g., allergic contact dermatitis). Occupational skin diseases have previously been identified as one of the leading causes of occupational illness within the United States workforce with many of the reported skin disorders being associated with chemical exposures.

The National Institute for Occupational Safety and Health (NIOSH) is dedicated to controlling and preventing workplace hazards including dermal exposures to chemicals. This document, Indexed Dermal Bibliography (1995-2007), is intended to serve as a resource guide for information on dermal issues within the workplace. The Indexed Dermal Bibliography has been structured to accommodate varying levels of technical background or formal training in identifying and controlling harmful skin exposures. The primary topics covered within the Indexed Dermal Bibliography include: (1) an overview of dermal exposures, (2) hazard identification, (3) exposure characterization, (4) health effects surveillance, (5) risk assessment, and (6) risk control management. This resource guide is not designed to be an exhaustive compilation of materials from the dermal exposure literature, but rather a representative list of available dermal exposure resources. The Indexed Dermal Bibliography contains, for the most part, review articles and an overview of educational information.

Christine M. Branche, Ph.D. /s

Acting Director, National Institute

for Occupational Safety and Health

Centers for Disease Control and Prevention 



\section{CONTENTS}

Foreword $\ldots \ldots \ldots \ldots \ldots \ldots \ldots \ldots \ldots \ldots \ldots \ldots \ldots \ldots \ldots \ldots$

Acknowledgments........................... ix

Abbreviations and Acronyms $\ldots \ldots \ldots \ldots \ldots \ldots \ldots \ldots \ldots \ldots \ldots \ldots$

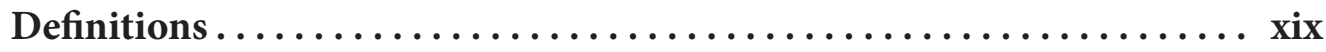

Quick Guide to Using the Indexed Dermal Bibliography......... xxi

CHAPTER 1: Introduction...................... 1

$1.1 \quad$ Background $\ldots \ldots \ldots \ldots \ldots \ldots \ldots \ldots \ldots \ldots \ldots \ldots \ldots \ldots \ldots \ldots \ldots \ldots$

1.2 Purpose of the Indexed Dermal Bibliography........... 1

1.3 Intended Uses and Audiences .................. 2

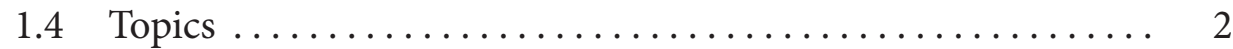

CHAPTER 2: Indexed Dermal Bibliography Development and Format 3

2.1 Criteria for Selection of Resources . . . . . . . . . . . . . . 3

2.2 Resource Review Process .......................... 4

2.3 Indexed Dermal Bibliography Contents............. 4

2.4 Indexed Dermal Bibliography Format ............ 5

2.5 Obtaining Resources.................... 6

CHAPTER 3: Resources for the General Audience ............ 7

3.1 Introduction .............................. 7

3.2 Resources for the General Audience by Topic............ 7

Topic 3A. Overview of Skin Exposures to Chemicals ....... 9

Topic 3B. Characterization of Exposure Condition (Exposure Characterization).......... 13

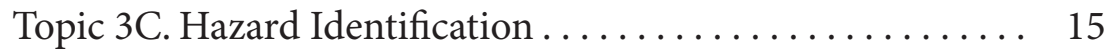

Topic 3D. Risk Assessment-Evaluating the Presence of Harmful Chemicals .................. 18

Topic 3E. Risk Management-Skin Exposure Risk Reductions .................. 19

CHAPTER 4: Resources for the Professional Audience........... 25

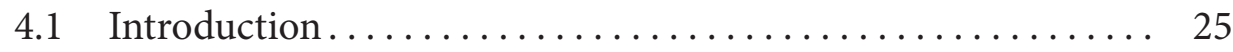

4. 2 Resources for the Professional Audience by Topic ......... 25 
Topic 4A. Overview of the Investigation and Control of Occupational Skin Exposures........... 27

Topic 4B. Surveillance and Clinical Aspects ......... 36

Topic 4C. Exposure Characterization ............ 41

Topic 4D. Hazard Identification from Toxicological Studies or Modeling.................... 53

Topic 4E. Risk Assessment . . . . . . . . . . . . . . . 66

Topic 4F. Risk Management..................... 69

CHAPTER 5: Overall Information Availability .............. 75

Appendix A: $\quad$ Full Resource Citations and Summaries.......... 79 


\section{ACKNOWLEDGMENTS}

The Indexed Dermal Bibliography was developed in the Education and Information Division (EID), by Paul Schulte, Ph.D., Director; Chen-Peng Chen, Ph.D.;Thomas J. Lentz, Ph.D.; Dawn Tharr; and G. Scott Dotson, Ph.D. from material produced under contract \# 200-2000-08017 with Westat. For contributions to the technical content and review of this document, the authors gratefully acknowledge the following NIOSH personnel:

Heinz W. Ahlers, J.D., National Personal Protective Technology Laboratory (NPPTL), NIOSH

Gregory A. Day, Ph.D., Division of Respiratory Disease Studies (DRDS), $\mathrm{NIOSH}$

Chad Dowell, Division of Surveillance, Hazard Evaluations and Field Studies (DSHEFS), NIOSH

Charles L. Geraci, Ph.D., EID, NIOSH

Cynthia Hines, DSHEFS, NIOSH

Larry Reed, DSHEFS, NIOSH

John P. Sestito, J.D., DSHEFS, NIOSH

Sid Soderholm, Ph.D., Office of the Director (OD), NIOSH

Douglas Trout, M.D., DSHEFS, NIOSH

The authors wish to thank Vanessa Becks, Gino Fazio, and Cathy Rotunda for their editorial support and contributions to the design and layout of this document.

Special appreciation is expressed to the following individuals and organizations for serving as independent external reviewers and providing comments that contributed to the development of this document:

Ben Hayes, M.D., Ph.D., Adjunct Clinical Instructor, Division of Dermatology, Vanderbilt School of Medicine

Sanja Kezic, Ph.D., Professor, Coronel Institute of Occupational Health, Academic Medical Center, University of Amsterdam 
Youcheng Liu, M.D., Sc.D., Assistant Professor, Department of Preventive Medicine and Environmental Health, College of Public Health, University of Kentucky

Scott P. Schneider, Division Director, Occupational Safety and Health Division, Laborers' Health \& Safety Fund of North America

James Taylor, M.D., Section Head, Industrial Dermatology, The Cleveland Clinic 


\section{ABBREVIATIONS AND ACRONYMS}

AAFP

ACC

ACGIH

AFL-CIO

AID

AIHA

ANOM

ANSI

API

ASA

ASTM

ATSDR

BAT values

BEELs

BEIs

BLS

CA DIR

CAS

CBD

CCOHS

CCP

CDC

CEB
American Academy of Family Physicians

American Chemistry Council

American Conference of Governmental Industrial Hygienists

American Federation of Labor and Congress of Industrial Organizations

allergic and irritant dermatitis

American Industrial Hygiene Association

analysis of means

American National Standards Institute

Alliance for the Polyurethanes Industry

American Skin Association

American Society for Testing and Materials

Agency for Toxic Substances and Disease Registry

biological tolerance values

biological environmental exposure limits

biological exposure indices

Bureau of Labor Statistics

California Department of Industrial Relations

chemical abstract service

chronic beryllium disease

Canadian Centre for Occupational Health and Safety

carbonless copy paper

Centers for Disease Control and Prevention

Chemical Engineering Branch of the USEPA's OPPTS 


\begin{tabular}{|c|c|}
\hline CEFIC & European Chemical Industry Council \\
\hline CFD & computational fluid dynamics \\
\hline CFR & Code of Federal Regulations \\
\hline ChemIDplus & $\begin{array}{l}\text { Free, web-based search system to access chemical } \\
\text { substances cited in the National Library of Medicine } \\
\text { databases }\end{array}$ \\
\hline CICADs & $\begin{array}{l}\text { Concise International Chemical Assessment } \\
\text { Documents }\end{array}$ \\
\hline CIS & $\begin{array}{l}\text { International Occupational Safety and Health Infor- } \\
\text { mation Centre (Centre international d'informations } \\
\text { de sécurité et santé au travail) }\end{array}$ \\
\hline CMAQ & Community Multiscale Air Quality Model \\
\hline COSHH & Control of Substances Hazardous to Health \\
\hline CPC & chemical protective clothing \\
\hline CPFB & chloropentafluorobenzene \\
\hline CPL & compliance directives \\
\hline CPI & Center for the Polyurethane Industry \\
\hline CPWR & $\begin{array}{l}\text { Center for Construction Research and Training } \\
\text { (Formerly known as the Center to Protect Workers' } \\
\text { Rights) }\end{array}$ \\
\hline CrVI & hexavalent chromium \\
\hline DCB & dichlorobenzene \\
\hline DEET & N,N-diethyl-m-toluamide \\
\hline DERMDAT & dermal exposure measurements \\
\hline DERP & Dermal Exposure Research Program \\
\hline DFP & diisopropylfluorphosphate \\
\hline DHHS & Department of Health and Human Services \\
\hline DLI & Department of Labor and Industries \\
\hline
\end{tabular}




$\begin{array}{ll}\text { DMF } & \text { N,N-dimethylformamide } \\ \text { DMSO } & \text { dimethylsulfoxide } \\ \text { DOEL } & \text { dermal occupational exposure limit } \\ \text { DP-PBPK } & \begin{array}{l}\text { Distributed parameter-physiologically based pharma- } \\ \text { cokinetic models }\end{array} \\ \text { DREAM } & \text { DeRmal Exposure AssessMent } \\ \text { EASE } & \text { Estimation Assessment of Substance Exposure mode } \\ \text { ECETOC } & \begin{array}{l}\text { European Centre for Ecotoxicology and Toxicology of } \\ \text { Chemicals }\end{array} \\ \text { eLCOSH } & \begin{array}{l}\text { Electronic Library of Construction Occupational Safe- } \\ \text { ty and Health }\end{array} \\ \text { EPIDERM } & \text { Experience of the British dermatologists } \\ \text { ERDEM } & \text { Exposure-Related Dose-Estimating Model } \\ \text { ERG } & \text { Emergency Response Guidebook } \\ \text { ERPG } & \text { Emergency Response Planning Guidelines } \\ \text { EVOH } & \text { ethylene vinyl alcohol } \\ \text { EXTOXNET } & \text { EXTension TOXicology NETwork } \\ \text { FAQs } & \text { frequently asked questions } \\ \text { FDA } & \text { Food and Drug Administration } \\ \text { FIFRA } & \text { Federal Insecticide, Fungicide, and Rodenticide Act } \\ \text { FIOH } & \text { Finnish Institute of Occupational Health } \\ \text { FRAMES-3MRA } & \begin{array}{l}\text { Framework for Risk Analysis in Multimedia Environ- } \\ \text { mental Systems-Multimedia, Multipathway, Multire- } \\ \text { ceptor Risk Assessment }\end{array} \\ \text { FTIR } & \text { Fourier transform infrared spectroscopy } \\ \text { Health and Safety Commission }\end{array}$


HSE

IARC

ICCVAM

ICSC

ID

IDLHs

ILO

INRS

IOM

IPCS

IPPSF

IRIS

ISEA

IT

LAS

LLNA

MAK

MDA

MDI

MMA

MMGs

NASD

NERL

NIEHS

NIOSH
Health and Safety Executive

International Agency for Research on Cancer

Interagency Coordinating Committee on the Validation of Alternative Methods

International Chemical Safety Card

identifier

Immediately Dangerous to Life and Health values

International Labour Office

l'Institut National de Recherche et de Sécurité

Institute of Medicine

International Programme on Chemical Safety

Isolated Perfused Porcine Skin Flap Model

Integrated Risk Information System

International Safety Equipment Association

intrinsic toxicity

linear alkibenzene sulfonate

local lymph node assay

maximum allowable concentration

4,4-methylene dianiline

Methylene diphenyl 4,4'-diisocyanate

methyl methacrylate

medical management guidelines

National Ag Safety Database

The USEPA's National Exposure Research Laboratory

National Institute of Environmental Health Sciences

National Institute for Occupational Safety and Health 


$\begin{array}{ll}\text { NIOSHTIC } & \begin{array}{l}\text { National Institute for Occupational Safety \& Health } \\ \text { Technical Information Center (database) }\end{array} \\ \text { NLM } & \text { National Library of Medicine } \\ \text { NORA } & \text { National Occupational Research Agenda } \\ \text { NOSQ } & \text { Nordic Occupational Skin Questionnaire } \\ \text { NPL } & \text { National Priorities List } \\ \text { NRL } & \text { natural rubber latex } \\ \text { NRMCA } & \text { National Ready Mix Concrete Association } \\ \text { NTIS } & \text { National Technical Information Service } \\ \text { OCD } & \text { occupational contact dermatitis } \\ \text { OECD } & \text { Organisation for Economic Co-operation and } \\ \text { OELs } & \text { Development } \\ \text { OH } & \text { occupational exposure limits } \\ \text { OPPTS } & \text { Ohio } \\ \text { OPRA } & \text { USEPA Office of Prevention, Pesticides and Toxic } \\ \text { OR } & \text { Substances } \\ \text { ORDHS } & \text { occupational physicians reporting activity } \\ \text { OR-OSHA } & \text { Oregon } \\ \text { OSH } & \text { Oregon Department of Human Services } \\ \text { OSHA } & \text { Oregon Occupational Safety and Health Division } \\ \text { OWIIPP } & \text { occupational safety and health } \\ \text { PAHs } & \text { Occupational Safety and Health Administration } \\ \text { PAR } & \text { progon Worker Illness and Injury Prevention } \\ & \text { polyaromatic hydrocarbons } \\ \text { PBsional acceptable residues }\end{array}$




$\begin{array}{ll}\text { PCBs } & \text { polychlorinated biphenyls } \\ \text { PDA } & \text { personal digital assistant } \\ \text { PDF } & \text { portable document format } \\ \text { PELs } & \text { permissible exposure limits } \\ \text { PHS } & \text { Public Health Service } \\ \text { PPE } & \text { personal protective equipment } \\ \text { pPm } & \text { part per million } \\ \text { PVA } & \text { polyvinylalcohol } \\ \text { PVC } & \text { polyvinylchloride } \\ \text { QSAR } & \text { quantitative structure-activity relationships } \\ \text { QSPRs } & \text { quantitative structure property relationships } \\ \text { RA } & \text { sulfate ricinolei acid } \\ \text { RAGS } & \text { Risk Assessment Guidance for Superfund } \\ \text { RCRA } & \text { Resource Conservation and Recovery Act } \\ \text { RD } & \text { low-level sulfur mustard } \\ \text { REACH } & \text { registration, evaluation, authorisation and restriction } \\ & \text { of chemical substances } \\ \text { RELs } & \text { recommended exposure limits } \\ \text { RISKofDERM } & \text { Risk assessment of occupational dermal exposure to } \\ \text { chemicals } & \text { Registry of Toxic Effects of Chemical Substances } \\ \text { RTECS } & \text { Secretariat of Communications and Transportation of } \\ \text { SCT } & \text { Mexico } \\ \text { SENSOR } & \text { Risks } \\ \text { SHARP } & \text { sentinel Event Notification System for Occupational } \\ & \end{array}$




$\begin{array}{ll}\text { SHEDS } & \text { Stochastic Human Exposure and Dose Simulation } \\ \text { SIS } & \text { Dodel } \\ \text { SMSEs } & \text { Division of Specialized Information Services } \\ \text { SRC } & \text { small- and medium-sized enterprises } \\ \text { SRP } & \text { Syracuse Research Corporation } \\ \text { STEL } & \text { Scientific Review Panel } \\ \text { TCDD } & \text { short-term exposure limit } \\ \text { TCE } & 2,3,7,8-\text { Tetrachlorodibenzo-p-Dioxin } \\ \text { TDI } & \text { trichloroethylene } \\ \text { TEHIP } & \text { Toluene diisocyanate } \\ \text { TLVs } & \text { Toxicology and Environmental Health Information } \\ \text { TOXLINE } & \text { Program } \\ \text { TOXNET } & \text { threshold limit values } \\ \text { TSCA } & \text { Toxicology Literature Online } \\ \text { TSCATS } & \text { Toxicology Data Network } \\ \text { TWA } & \text { Toxic Substances Control Act } \\ \text { USACHPPM } & \text { Toxic Substances Control Act Test Submission } \\ \text { UNEP } & \text { Database } \\ \text { USDOT } & \text { time weighted average } \\ \text { USEPA } & \text { U.S. Army Center for Health Promotion and Preven- } \\ \text { WHO } & \text { Unive Medicine } \\ \text { WOCs } & \text { United Stated Department of Transportation } \\ \text { United States Environmental Protection Agency } \\ \text { volatile organic compounds } \\ \text { Washington Department of Labor and Industry } \\ \text { Whith Organization }\end{array}$


WISER

WEELs
Wireless Information System for Emergency Responders

Workplace Environmental Exposure Level handbook 


\section{DEFINITIONS}

Article ID:

Audiences:

Chemical:

Citation:

Educational material:

Mixtures:

Number of references:

Resource type:
The unique ID assigned to every resource found in the Indexed Dermal Bibliography.

Whether the reference was written primarily for a general or professional audience. General audience is defined as those who have limited technical background or formal training in identifying and controlling hazardous skin exposures. Professional audiences typically utilize technical information for evaluating, recognizing, and controlling harmful skin exposures.

The broad chemical classes-whether raw, intermediate, or final products - to which the resource pertains or specifically addresses (e.g., abrasives, pesticides, PCBs, etc.).

The information (e.g., author, title, journal, volume) needed to obtain the reference.

Whether the material seem to have been developed with the primary focus of educating the workforce or general audiences.

Industries/Occupations: The broad categories of occupations and industries to which the resource states that it pertains or specifically addresses (e.g., agriculture, construction, mining).

Whether the references addresses the topic of chemical mixtures and dermal exposure.

The number of references cited. NOTE: The total number for books was determined by summing the number of references in each chapter.

Type of resource (e.g., journal, book, magazine, Web page). NOTE: Web sites may contain multiple types of resources not listed. These will be summarized in the summary text. 
Specific chemicals:

Specific process:

Summary:

Topics addressed:
Specific chemicals to which the resource pertains or specifically addresses.

Specific occupations, jobs, or tasks, if any, addressed by the reference that are not listed or detailed in the industries/occupations drop-down list.

A summary of the document written with a focus on occupational dermal exposures. The summary is not identical to the reference's abstract. Web site summaries may summarize links to resources within the Web site.

The list of broad topics addressed by the references (e.g., health effects, exposure characterization). Most resources contain information about multiple topics and subtopics. This list provides an overview of the kinds of information that can be found within the resource. Specific topics for general audiences are explained in full in Chapter 3 and for professional audiences in Chapter 4. 


\section{QUICK GUIDE TO USING THE INDEXED DERMAL BIBLIOGRAPHY}

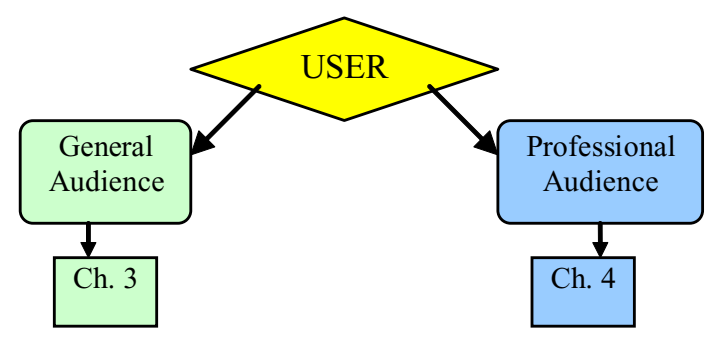

\section{STEP 1. AUDIENCE}

Select General or Professional Audience. (See Section 1.3 for definition of each audience.)

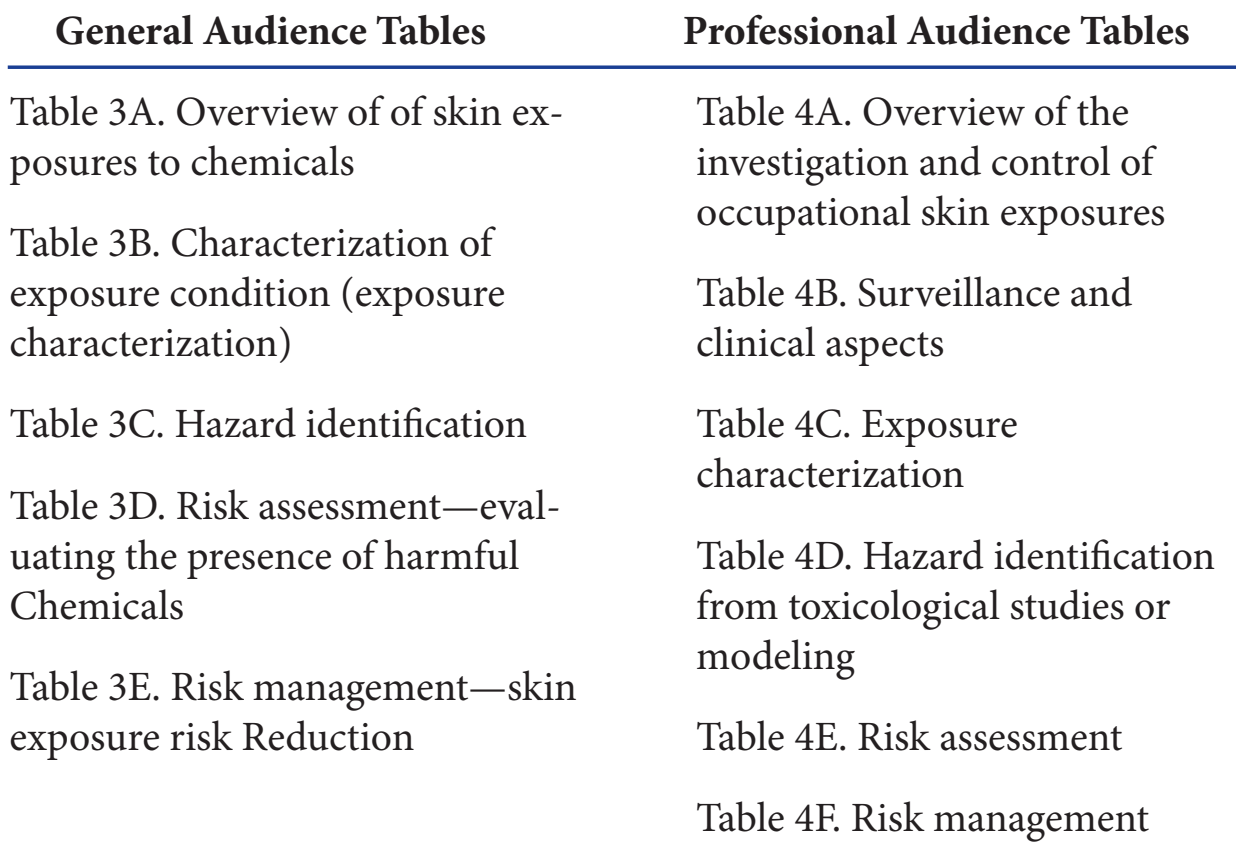

\section{STEP 2. TOPIC OF INTEREST}

Select table with main topic of interest, as defined in Chapters 3 and 4. 


\section{STEP 3. RESOURCE TABLES}

Go to Table and select resource(s) of interest based on:

1. Resource type

2. Title, Author

3. Year of publication
4. Sub-topic of interest, as defined in Chapters 3 and 4.

5. Article ID number for resource(s) of interest.

Proceed to Appendix A.

\begin{tabular}{|c|c|c|c|c|c|c|}
\hline & & & & \multicolumn{3}{|c|}{ Subtopics } \\
\hline Resource type & ID & Title, author & Yr & A.1 & A. 2 & A.3 \\
\hline Book/monograph, whole & 478 & $\begin{array}{l}\text { Essentials of Occupational Skin } \\
\text { Management, PackhamCL }\end{array}$ & 1999 & $\checkmark$ & $\checkmark$ & $\checkmark$ \\
\hline \multirow[t]{2}{*}{ Brochure, pamphlet } & 107 & $\begin{array}{l}\text { Do you know about... the health } \\
\text { hazards of benzene?, }\end{array}$ & 2000 & $\checkmark$ & & \\
\hline & 108 & $\begin{array}{l}\text { Do you know... the hazards of } \\
\text { solvents? }\end{array}$ & 2000 & $\checkmark$ & $\checkmark$ & \\
\hline Guideline & 402 & $\begin{array}{l}\text { What You Need to Know About } \\
\text { Occupational Exposure to Metal- } \\
\text { working Fluids, }\end{array}$ & 1998 & & $\checkmark$ & \\
\hline \multirow[t]{2}{*}{ Magazine article } & 2 & $\begin{array}{l}\text { Skin Care: Starting from Scratch, } \\
\text { Nash,James L. }\end{array}$ & 2000 & $\checkmark$ & & \\
\hline & 3 & $\begin{array}{l}\text { Dealing With Dermal Allergies and } \\
\text { Skin Reactions, Groce,Don }\end{array}$ & 2000 & $\checkmark$ & $\checkmark$ & \\
\hline
\end{tabular}

\section{STEP 4. APPENDIX A-FULL RESOURCE CITATION AND SUMMARY}

In Appendix A, look up the ID numbers for resource(s) of interest. Resources are sorted numerically by ID number.

Evaluate the resource(s) based on the citation and summary information provided. 


\section{CHAPTER 1 Introduction}

The Indexed Dermal Bibliography (1995-2007) is a tool that directs workers, employers, industrial hygienists, researchers, and policy makers to information resources on occupational skin exposures to chemicals, including health effects surveillance, exposure characterization, hazard identification, risk assessment, and risk control and management.

\subsection{Background}

More than 13 million workers in the United States are potentially exposed to chemicals at work via the skin. A worker's skin may be exposed to harmful chemicals through direct contact with contaminated surfaces, deposition of aerosols, and immersion in or splashes from liquids. Some chemicals cause contact dermatitis via direct skin contact. Contact dermatitis is one of the more frequently reported occupational illnesses, accounting for $10 \%-15 \%$ of all occupational diseases, at an estimated annual cost in the United States (U.S.) of at least $\$ 1$ billion.

Many chemicals readily pass through the skin (called dermal penetration). Some of these chemicals are then taken up into the blood stream or by skin or immune cells (this is called dermal absorption). Dermal absorption can cause systemic health effects or can contribute to the effects of chemicals absorbed in the lungs by inhalation. Chemicals are often absorbed through the skin without being noticed by the worker. In some cases, the skin is a more significant route of exposure than the respiratory tract. This is particularly true for nonvolatile chemicals that are relatively toxic and that remain on work surfaces for long periods of time.

\subsection{Purpose of the Dermal Resource Guide}

The Indexed Dermal Bibliography is designed to serve as a resource for information on dermal exposure for those who work in (1) at-risk occupations, (2) positions to investigate or control worker skin exposure to harmful chemicals, and (3) research settings or positions to set policy on dermal exposures. The Indexed Dermal Bibliography provides lists and descriptions of resources by topic for people looking for specific information on dermal exposure anticipation, recognition, evaluation, and control.

The Indexed Dermal Bibliography is not designed to be an exhaustive listing of materials from the dermal exposure literature, but rather a representative list of available dermal exposure resources. The Indexed Dermal Bibliography contains 
review articles and summaries of educational information. Individual research studies are not included here. In addition, the accuracy of information presented in the references has not been evaluated.

\subsection{Intended Uses and Audiences}

The Indexed Dermal Bibliography is designed to provide descriptions of resources available for two different audiences: the general audience and the professional audience.

Resources for the general audience are for those who have limited technical background or formal training in identifying and controlling harmful skin exposures. The general audience may include workers, small business employers, supervisors, worksite owners, insurers, and manufacturers of industrial chemicals.

Resources for the professional audience are for those who typically use technical information for evaluating, recognizing, and controlling harmful skin exposures. The professional audience may include industrial hygienists, occupational epidemiologists, dermatologists, occupational physicians and nurses, academic researchers, toxicologists, and policy makers.

In some cases resources for these two audiences are not mutually exclusive. General audience members are encouraged to look at the professional resources when they are interested in more detailed or technical information. Conversely, professionals looking for background information for training, education, or communication purposes may find relevant information in the resources for the general audience.

\subsection{Topics}

The Indexed Dermal Bibliography lists resources that address a number of broad topics. The topics differ somewhat between general and professional audiences, but typically address the following:

- Overview of dermal exposure.

- Surveillance and clinical aspects of dermal exposures.

- Dermal exposure characterization.

- Dermal hazard identification.

- Dermal exposure risk assessment.

- Dermal exposure risk management.

These topics were specified during a workshop held at the International Conference on Occupational and Environmental Exposure of Skin to Chemicals: Science and Policy, held September 11, 2002 in Crystal City, VA. The topics are defined and discussed in more detail in Chapters 3 and 4. 


\section{CHAPTER 2 Indexed Dermal Bibliography Development and Format}

\subsection{Criteria for Selection of Resources}

Resources listed in the Indexed Dermal Bibliography were identified by first conducting an electronic search of review articles on dermal exposure topics published in English between 1995 and 2007. Key words were identified, grouped, and used to search nine different bibliographic databases (Combined Health Information Database, Cumulative Index to Nursing and Allied Health Literature, Enviroline, Gale Group Health \& Wellness Database ${ }^{\mathrm{SM}}$, Health Source-Consumer Edition, National Technical Information Service (NTIS), PubMed/Medline, Wilson Applied Science \& Technology Abstracts, and WorldCat) and three search engines for government documents (FirstGov.gov, Google UncleSam. com, and SearchGov.com). Additional resources were identified from government guidelines, significant Web sites, and suggestions from dermal exposure experts. These efforts resulted in identification of over 600 resources potentially suitable for inclusion in this guide. Of these, 229 resources were retained in the final version of this guide.

In order to select the resources for inclusion in the Indexed Dermal Bibliography, reviewers first screened the abstracts and other available bibliographic information for each of the identified resources for relevance to the Indexed Dermal Bibliography. Based on the screening, each resource was either recommended, not recommended, or potentially recommended for inclusion in the guide. For a resource to be considered for inclusion, it needed to meet the following criteria:

- Be published in English between 1995 and 2007. (In rare instances, review articles or resources published before 1995 were selected for review when they were considered key resources.)

- Cover occupational exposures that are primarily via dermal pathways.

- Cover dermal exposures to chemical hazards, rather than physical or biological hazards.

- Deal primarily with human exposures or animal studies directly related to human exposures.

- Be nonclinical and designed for the nonphysician audience.

- Be a review article or a meta-analysis of primary research studies. 


\subsection{Resource Review Process}

Following screening, copies of recommended resources were obtained and reviewed for inclusion in the Indexed Dermal Bibliography. All resources that were included in the Guide were reviewed more fully to summarize the content of the resource. For each of the references, the following information was gathered:

- Industry covered by the resources, including specific industries or occupations when they were discussed in-depth.

- Chemical classes covered in the resource, including any specific chemicals that were covered in-depth.

- Discussion of any issues surrounding dermal exposure to chemical mixtures.

- Audience-professional or general (see Section 1.3. above).

- Major topics associated with dermal exposure covered in the resources.

In addition, a brief summary of each resource was written, or, in the case of Web sites and Web pages, key information found within the resource was highlighted and as appropriate, summarized.

The draft Indexed Dermal Bibliography underwent an external review process which included review by the general public. Minor edits and additions were made in response to reviewer comments. Several newer articles were also added during the review process. Of the original 600+ resources, 229 resources met the previously described criteria and are included in the guide.

\subsection{Indexed Dermal Bibliography Contents}

The 229 resources in the Indexed Dermal Bibliography include review articles published in peer reviewed journals, occasional primary journal articles, books, book chapters, brochures/pamphlets, databases, government policies and regulations, guidelines, magazine articles, technical reports, and Web sites and Web pages. Individual research studies are not included here.

Web sites and Web pages were treated somewhat differently with respect to citations and summaries, given the unique nature of their content. First to clarify, Web sites contain a variety resources, including Web pages and downloadable documents, data files, and databases. Web sites are somewhat analogous to a book, with the Web site being the book and the Web pages being chapters within the book. However, Web sites are more complicated than books because Web pages can be much more varied in content and format than chapters. In 
addition, Web site and Web page content is not necessarily static content, as is the case with printed material. Information available on a Web site can change or be updated over time, can be removed, or can be moved to a different location within the Web site. It is not always clear on examination whether or not this has been done. Finally, the Web sites reviewed and included in the Indexed Dermal Bibliography contain a variety of independent information located throughout the Web sites as well as links to other Web sites. The advantage of resources available on Web sites is that they are instantaneously available and, for the most part, free, making Web sites an invaluable source of information for those addressing skin exposures in the field.

Web site summaries include general descriptions of Web pages, data files, and databases found within the Web site. The information cited in the Indexed Dermal Bibliography is based on the version of the Web site at the time it was reviewed.

\subsection{Indexed Dermal Bibliography Format}

The Indexed Dermal Bibliography is divided into six chapters and two appendices. The content of each is described below.

- Chapter 1: Background, purpose, target audiences, and topic areas.

- Chapter 2: $\quad$ Indexed Dermal Bibliography contents, format, and criteria for selection of resources.

- Chapter 3: General audience resources. The major topics covered are overview, exposure characterization, hazard identification, risk assessment, and risk management. Under each topic is a table listing those resources that include information on that topic, listed by resource type, ID number, author, date, and title.

- Chapter 4: $\quad$ Professional audience resources. The major topics covered are overview, surveillance and clinical aspects, exposure characterization, hazard identification, risk assessment, and risk management. Under each topic is a table listing those resources that include information on that topic, listed by resource type, ID number, author, date, and title.

- Chapter 5: Discussion of overall information availability.

- Appendix A: Full Resource Citations and Summaries. The appendix provides the citations and summaries of all resources in the Indexed Dermal Bibliography, sorted by resource 
ID number. The Web site summaries list Web pages, databases, and data files found at the site which contain useful information on dermal exposures.

\subsection{Obtaining Resources}

Resources can be obtained through a variety of means. Books, journals, and magazine articles can be obtained through subscribing libraries or through interlibrary loan. As needed, books may also be purchased through a variety of suppliers.

More and more review articles are either available for purchase online or are free. One convenient source for finding articles available online is PubMed/ Medline (http://www.ncbi.nlm.nih.gov/entrez/query.fcgi).

Some of the governmental and nongovernmental agency publications can also be found either for purchase or download online. Generally these can be located by typing in the report title using a search engine such as Google (www.google. com).

Web sites and Web pages can typically be accessed by the URL provided in Appendix A. If the URL link is no longer in use, Web pages or files may be searched by typing in key words on the Web site search engine. Alternatively, the table of contents links under the home page can be used. For Web resources described that do not have listed URLs, the resource might be found using the Web site search engine, typing in the name of the desired resource or keywords associated with the resource, and accessing the information. If the resource is still not found on the Web site or Web page, help can be solicited from the Web site masters, usually through a "help" or "contact us" Web page. 


\section{CHAPTER 3 \\ Resources for the General Audience}

\subsection{Introduction}

The resources identified below are appropriate for a general audience who wants background information on dermal exposures to chemicals. General audiences are those who desire to maintain a safe and healthful worksite, but have limited technical background or formal training in identifying and controlling hazardous skin exposures. The general audience may include workers, small business employers, supervisors, worksite owners, insurers, and manufacturers of industrial chemicals.

The resources presented in the tables are review articles published in peer-reviewed journals, as well as books, magazines, Web sites, regulatory guidelines, databases, brochures and other types of resources. These are not meant to be a comprehensive list of information available for the general audience, but rather a representative list of what is available. In addition, the accuracy of the information contained in any resource has not been evaluated.

General audience members who would like more detailed information on some of the topic areas are encouraged to also look at the resources identified for professional audiences in Chapter 4. For example, if a user would like more information on factors that influence exposure conditions (Table 3B, Subtopic B2), they could look in Chapter 4 at Table 4C under Subtopic C2, Description of Factors Influencing Exposure Conditions.

\subsection{Resources for the General Audience by Topic}

The following five tables list, for a general audience, resources covering each major topic related to occupational skin exposure to chemicals. The major topics are further divided into varying numbers of subtopics, each of which is represented in the columns on the right-hand side of the tables.

Descriptions of each topic and subtopics are provided before each table to assist users in deciding what kind of information they are interested in learning more about.

For the general audience, the five major topics and associated subtopics are:

Topic 3A. Overview of Skin Exposures to Chemicals

A.1. Occurrence of Skin Exposures in the Workplace 
A.2. Health Effects from Skin Exposures to Chemicals

A.3. Dermal Regulations and Skin Notations

Topic 3B. Characterization of Exposure Condition (Exposure Characterization)

B.1. Job/Tasks, Industries/Processes, or Chemicals with Skin Exposures

B.2. Factors that Influence Exposure Conditions

B.3. Protocols/Checklists to Characterize Exposure to Skin Hazards

Topic 3C. Hazard identification

C.1. Risk Phrases, Hazard Symbols, Skin Designations

C.2. Tables/Charts/Lists of Hazards for Specific Chemicals

C.3. Protocols/Checklists to Identify Skin Hazards in the Workplace

Topic 3D. Risk Assessment-Evaluating the Presence of Harmful Chemicals

D.1. Protocols/Checklists to Identify Exposure Risk

Topic 3E. Risk management-Skin Exposure Risk Reduction

E.1. Overview of Skin Exposure Control Options

E.2. Protocols/Checklists to Monitor Potential Exposures

E.3. "Best practices"/Guidelines/Recommendations

E.4. Guidelines/Recommendations for Post-exposure Skin Decontamination

Each of the five tables presented below include the following columns:

- Resource Type-book, brochure, journal article, Web site, etc.

- ID-unique number assigned to each resource and can be used to locate each resource in Appendix A. All resources in Appendix A are sorted alphabetically by resource type and then numerically by ID.

- Title, Author - title and author, if available, for each resource.

- Yr-the year of publication of the resource; for Web sites and Web pages, the year the Web site or Web page was reviewed for inclusion in the Indexed Dermal Bibliography.

- Subtopics - each subtopic addressed by a resource is checked in the appropriate column; subtopics are defined at the bottom of each table. 
A given resource may be repeated in multiple tables. This will happen when a resource provides information covering a variety of topics.

\section{Topic 3A. Overview of Skin Exposures to Chemicals}

Table 3A identifies resources that provide general background information on how skin exposure to chemicals in the workplace might cause health-related problems, as well as general information about health effects that can result from such exposures. Each checked box under subtopics indicates that that subject area is covered in the resource.

The following subtopics associated with exposure overview information are presented and defined below.

\section{Subtopic A.1. Occurrence of Skin Exposures in the Workplace}

These resources provide an overview of issues associated with the occurrence of skin exposures in the workplace.

\section{Subtopic A.2. Health Effects from Skin Exposures to Chemicals}

These resources contain general information on the health hazards associated with skin exposure to chemicals. Health effects from skin exposures to chemicals can vary ranging from local effects (e.g., irritation, burns, or skin breakdown) to allergic reactions, including both localized responses (e.g., hives) as well as more remote location responses (e.g., respiratory or lung effects).

\section{Subtopic A.3. Dermal Regulations and Skin Notations}

These resources contain information about regulations covering occupational skin exposure to chemicals. Unlike chemical inhalation hazards, there are currently no occupational exposure limits for skin exposures to chemicals. Instead, regulatory agencies assign a skin notation to a chemical to indicate that the chemical has the potential to contribute to the overall chemical exposure by absorption through the skin. Skin notations do not indicate whether a chemical can cause a localized response, only that skin exposure can contribute to overall exposure. The governmental Web sites listed contain information on applicable standards. In addition to regulatory information, some of these resources also contain lists of chemicals that have assigned skin notations. 


\section{GENERAL AUDIENCE \\ Table 3A. Overview of skin exposures to chemicals}

\begin{tabular}{|c|c|c|c|c|c|c|}
\hline \multirow[b]{2}{*}{ Resource type } & \multirow[b]{2}{*}{ ID } & \multirow[b]{2}{*}{ Title, author } & \multirow[b]{2}{*}{ Yr } & \multicolumn{3}{|c|}{ Subtopics } \\
\hline & & & & A. $1^{\star}$ & A.2 & A.3 \\
\hline $\begin{array}{l}\text { Book/monograph, } \\
\text { whole }\end{array}$ & 169 & $\begin{array}{l}\text { Essentials of occupational skin management, Pack- } \\
\text { ham CL }\end{array}$ & 1999 & $\checkmark$ & $\checkmark$ & $\checkmark$ \\
\hline \multirow[t]{8}{*}{$\begin{array}{l}\text { Brochure, } \\
\text { pamphlet }\end{array}$} & 7 & $\begin{array}{l}\text { Do you know about... the health hazards of benzene? } \\
\text { Occupational Health Department }\end{array}$ & 2000 & $\checkmark$ & $\checkmark$ & \\
\hline & 8 & $\begin{array}{l}\text { Did you know the hazards of solvents? Occupational } \\
\text { Health Department }\end{array}$ & 2000 & $\checkmark$ & $\checkmark$ & \\
\hline & 9 & $\begin{array}{l}\text { Assessing and managing risks at work from skin ex- } \\
\text { posure to chemical agents: Guidance for employers } \\
\text { and health and safety specialists, Health and Safety } \\
\text { Executive (HSE) }\end{array}$ & 2001 & $\checkmark$ & $\checkmark$ & $\checkmark$ \\
\hline & 12 & Choice of skin care products for the workplace, HSE & 2001 & & $\checkmark$ & \\
\hline & 24 & $\begin{array}{l}2005 \text { Emergency response planning guidelines } \\
\text { (ERPG) and workplace environmental exposure } \\
\text { level (WEEL) handbook, American Industrial Hy- } \\
\text { giene Association (AIHA) }\end{array}$ & 2005 & & & $\checkmark$ \\
\hline & 60 & $\begin{array}{l}\text { A safety and health practitioner's guide to skin pro- } \\
\text { tection, Center to Protect Workers' Rights (CPWR) }\end{array}$ & 2000 & $\checkmark$ & $\checkmark$ & $\checkmark$ \\
\hline & 62 & An employer's guide to skin protection, CPWR & 2005 & $\checkmark$ & $\checkmark$ & \\
\hline & 95 & $\begin{array}{l}\text { Quick selection guide to chemical protective clothing } \\
\text { (CPC), Forsberg K }\end{array}$ & 2002 & & & $\checkmark$ \\
\hline Guideline & 3 & $\begin{array}{l}\text { What you need to know about occupational exposure } \\
\text { to metalworking fluids, National Institute for Oc- } \\
\text { cupational Safety and Health (NIOSH) }\end{array}$ & 1998 & & $\checkmark$ & \\
\hline \multirow{2}{*}{$\begin{array}{l}\text { Journal article- } \\
\text { review, } \\
\text { meta-analysis }\end{array}$} & 43 & $\begin{array}{l}\text { Federal government regulation of occupational skin } \\
\text { exposure in the USA, Boeniger MF }\end{array}$ & 2003 & & & $\checkmark$ \\
\hline & 147 & $\begin{array}{l}\text { The value and limitations of protective gloves in } \\
\text { medical health service: Part III, Mellstrom GA }\end{array}$ & 1996 & & $\checkmark$ & \\
\hline
\end{tabular}

${ }^{\star}$ See footnotes at end of table.

(Continued) 


\section{GENERAL AUDIENCE \\ Table 3A (Continued). Overview of skin exposures to chemicals}

\begin{tabular}{|c|c|c|c|c|c|c|}
\hline \multirow[b]{2}{*}{ Resource type } & \multirow[b]{2}{*}{ ID } & \multirow[b]{2}{*}{ Title, author } & \multirow[b]{2}{*}{ Yr } & \multicolumn{3}{|c|}{ Subtopics } \\
\hline & & & & A. $1^{*}$ & A.2 & A.3 \\
\hline \multirow[t]{3}{*}{ Magazine article } & 75 & $\begin{array}{l}\text { Protecting the hand-skin barrier in the workplace, } \\
\text { Del Rosso J }\end{array}$ & 2000 & & $\checkmark$ & \\
\hline & 149 & Skin care: starting from scratch, Nash JL & 2000 & $\checkmark$ & & \\
\hline & 181 & Safe use of glutaraldehyde, Romano-Woodward D & 2000 & & $\checkmark$ & \\
\hline \multirow[t]{4}{*}{ Web page } & 19 & $\begin{array}{l}\text { Toxicological profile information sheet, Agency for } \\
\text { Toxic Substance and Disease Registry (ATSDR) }\end{array}$ & 2005 & $\checkmark$ & $\checkmark$ & $\checkmark$ \\
\hline & 21 & $\begin{array}{l}\text { Skin problems: How to protect yourself from job-re- } \\
\text { lated skin problems, American Academy of Family } \\
\text { Physicians (AAFP) }\end{array}$ & 2004 & $\checkmark$ & $\checkmark$ & \\
\hline & 205 & Health and safety zone, Unison & 2005 & $\checkmark$ & $\checkmark$ & \\
\hline & 220 & $\begin{array}{l}\text { Dermatitis: safety and health assessment and research } \\
\text { for prevention (SHARP), Washington Department } \\
\text { of Labour Industry (WADLI) }\end{array}$ & 2005 & $\checkmark$ & $\checkmark$ & \\
\hline \multirow[t]{6}{*}{ Web site } & 13 & $\begin{array}{l}\text { The American Skin Association, (ASA) [Home page], } \\
\text { ASA }\end{array}$ & 2005 & $\checkmark$ & $\checkmark$ & \\
\hline & 14 & $\begin{array}{l}\text { Montana Department of Labor and Industries- } \\
\text { Employment Relations, (MTDLI) [Home page], } \\
\text { MTDLI }\end{array}$ & 2005 & $\checkmark$ & $\checkmark$ & \\
\hline & 15 & $\begin{array}{l}\text { Occupational health and safety, [Web site] } 1105 \\
\text { Media, Inc. }\end{array}$ & 2006 & $\checkmark$ & & $\checkmark$ \\
\hline & 16 & $\begin{array}{l}\text { Center for the Polyurethanes Industry (CPI) [Home } \\
\text { page], American Chemistry Council (ACC) }\end{array}$ & 2006 & $\checkmark$ & $\checkmark$ & \\
\hline & 22 & AAFP [Home page], AAFP & 2005 & $\checkmark$ & $\checkmark$ & \\
\hline & 29 & Ansell Chemsafe [Home page], Ansell Chemsafe & 2005 & $\checkmark$ & & \\
\hline
\end{tabular}

${ }^{\star}$ See footnotes at end of table.

(Continued) 
GENERAL AUDIENCE

Table 3A (Continued). Overview of skin exposures to chemicals

\begin{tabular}{|c|c|c|c|c|c|c|}
\hline \multirow[b]{2}{*}{ Resource type } & \multirow[b]{2}{*}{ ID } & \multirow[b]{2}{*}{ Title, author } & \multirow[b]{2}{*}{ Yr } & \multicolumn{3}{|c|}{ Subtopics } \\
\hline & & & & A. $1^{\star}$ & A.2 & A.3 \\
\hline \multirow[t]{11}{*}{$\begin{array}{l}\text { Web site } \\
\text { (Continued) }\end{array}$} & 58 & $\begin{array}{l}\text { Canadian Centre for Occupational Health and Safety } \\
\text { (CCOHS) [Home page], CCOHS }\end{array}$ & 2005 & $\checkmark$ & $\checkmark$ & \\
\hline & 59 & CPWR [Web page], CPWR & 2006 & $\checkmark$ & & \\
\hline & 63 & $\begin{array}{l}\text { Electronic Library of Construction Occupational } \\
\text { Safety and Health (eLCOSH) [Web site], CPWR }\end{array}$ & 2005 & $\checkmark$ & $\checkmark$ & \\
\hline & 88 & $\begin{array}{l}\text { European Agency for Safety and Health and Work } \\
\text { [Home page], European Agency for Safety and } \\
\text { Health and Work }\end{array}$ & 2005 & $\checkmark$ & $\checkmark$ & $\checkmark$ \\
\hline & 111 & $\begin{array}{l}\text { International Brotherhood of Teamsters [Home } \\
\text { page], International Brotherhood of Teamsters }\end{array}$ & 2006 & $\checkmark$ & $\checkmark$ & \\
\hline & 112 & $\begin{array}{l}\text { International Labor Organization (ILO) [Home } \\
\text { page], ILO }\end{array}$ & 2005 & $\checkmark$ & $\checkmark$ & $\checkmark$ \\
\hline & 156 & $\begin{array}{l}\text { National Ready Mixed Concrete Association } \\
\text { (NRMCA) [Home page], NRMCA }\end{array}$ & 2006 & $\checkmark$ & & \\
\hline & 162 & $\begin{array}{l}\text { Occupational Safety and Health Administration } \\
\text { (OSHA) [Home page], OSHA }\end{array}$ & 2005 & & $\checkmark$ & $\checkmark$ \\
\hline & 165 & Oregon OSHA (OR-OSHA) [Home page], OR-OSHA & 2006 & & $\checkmark$ & $\checkmark$ \\
\hline & 174 & $\begin{array}{l}\text { Portland Cement Association (PCA) [Home page], } \\
\text { PCA }\end{array}$ & 2006 & $\checkmark$ & $\checkmark$ & \\
\hline & 207 & $\begin{array}{l}\text { United States Army Center for Health Promotion \& } \\
\text { Preventive Medicine (USACHPPM) [Home page], } \\
\text { USACHPPM }\end{array}$ & 2006 & $\checkmark$ & & \\
\hline
\end{tabular}

${ }^{\star}$ A.1=Occurrence of Skin Exposures in the Workplace; A.2=Health Effects from Skin Exposures to Chemicals; A.3 $=$ Dermal Regulations and Skin Notations 


\section{Topic 3B. Characterization of Exposure Condition (Exposure Characterization)}

Exposure characterization is the process of describing the qualities of a given environment that may influence exposure. These conditions may include the following:

- Source of the chemical.

- Amount of chemical a worker is exposed to, the amount of time a worker is exposed in a work day, and how often a worker is exposed in a given week.

- Routes of the exposure to the chemical, such as exposure through the skin, lungs, or through the mouth if food or drink is contaminated.

- Chemical and physical properties of the chemical.

- Work practices (i.e., or how work using the chemical is performed).

\section{Subtopic B.1. Job/Tasks, Industries/Processes, or Chemicals with Skin Exposures}

These resources may contain information on industries, processes, or jobs that are prone to expose workers to chemicals that are harmful to the skin. If available, the specific chemicals or classes of chemicals involved in the skin exposures are listed in the resource summary in Appendix A.

\section{Subtopic B.2. Factors that Influence Exposure Conditions}

These resources contain descriptions of factors that influence exposure conditions. Exposure conditions not only include the working conditions for a job being performed, but also the specific factors that influence exposure. Factors that can influence exposure conditions include the following: (1) intensity or amount of the exposure and (2) duration of exposure and frequency in a given day, week, month, or year. Other factors that influence exposure conditions include what control measures are in place to help reduce exposure, including engineering controls, work practices that either increase or decrease exposures, and the use of personal protective equipment (PPE) such as gloves. For example, two different workplaces with workers performing the same task can have different exposure conditions based on what kind of control measures are in use at each workplace.

\section{Subtopic B.3. Protocols/Checklists to Characterize Exposure to Skin Hazards}

The resources in this subtopic provide protocols and checklists that can be followed to characterize exposures to skin hazards. Only those resources with protocols or checklists specific to dermal exposure characterization are checked here. 
GENERAL AUDIENCE Table 3B. Characterization of exposure condition (exposure characterization)

\begin{tabular}{|c|c|c|c|c|c|c|}
\hline \multirow[b]{2}{*}{ Resource type } & \multirow[b]{2}{*}{ ID } & \multirow[b]{2}{*}{ Title, author } & \multirow[b]{2}{*}{ Yr } & \multicolumn{3}{|c|}{ Subtopics } \\
\hline & & & & B. $1^{\star}$ & B. 2 & B.3 \\
\hline \multirow[t]{2}{*}{$\begin{array}{l}\text { Book/monograph, } \\
\text { whole }\end{array}$} & 169 & $\begin{array}{l}\text { Essentials of occupational skin management, Pack- } \\
\text { ham CL }\end{array}$ & 1999 & $\checkmark$ & $\checkmark$ & \\
\hline & 185 & Dermal exposure assessment, Sahmel J & 2006 & $\checkmark$ & $\checkmark$ & \\
\hline \multirow[t]{3}{*}{ Brochure, pamphlet } & 9 & $\begin{array}{l}\text { Assessing and managing risks at work from skin ex- } \\
\text { posure to chemical agents: Guidance for employers } \\
\text { and health and safety specialists, HSE }\end{array}$ & 2001 & $\checkmark$ & & \\
\hline & 60 & $\begin{array}{l}\text { A safety and health practitioner's guide to skin protec- } \\
\text { tion, CPWR }\end{array}$ & 2000 & $\checkmark$ & $\checkmark$ & \\
\hline & 62 & An employer's guide to skin protection, CPWR & 2005 & $\checkmark$ & $\checkmark$ & \\
\hline Guideline & 3 & $\begin{array}{l}\text { What you need to know about occupational exposure } \\
\text { to metalworking fluids, NIOSH }\end{array}$ & 1998 & & $\checkmark$ & \\
\hline \multirow[t]{2}{*}{ Web page } & 205 & Health and safety zone [Home page], Unison & 2005 & $\checkmark$ & & \\
\hline & 220 & $\begin{array}{l}\text { Dermatitis: Safety and health assessment and research } \\
\text { for prevention (SHARP) [Home page], WADLI }\end{array}$ & 2005 & $\checkmark$ & $\checkmark$ & \\
\hline \multirow[t]{6}{*}{ Web site } & 14 & $\begin{array}{l}\text { MT DLI Employment Relations [Home page], MT } \\
\text { DLI }\end{array}$ & 2005 & $\checkmark$ & & \\
\hline & 15 & $\begin{array}{l}\text { Occupational health and safety [Web site], } 1105 \\
\text { Media, Inc. }\end{array}$ & 2006 & $\checkmark$ & & $\checkmark$ \\
\hline & 16 & CPI [Web site], ACC & 2006 & $\checkmark$ & $\checkmark$ & \\
\hline & 22 & AAFP [Home page], AAFP & 2005 & $\checkmark$ & & \\
\hline & 58 & CCOHS [Home page], CCOHS & 2005 & $\checkmark$ & $\checkmark$ & \\
\hline & 63 & eLCOSH [Web site], CPWR & 2005 & $\checkmark$ & $\checkmark$ & $\checkmark$ \\
\hline
\end{tabular}

*See footnotes at end of table.

(Continued) 
GENERAL AUDIENCE

Table 3B (Continued). Characterization of exposure condition (exposure characterization)

\begin{tabular}{|c|c|c|c|c|c|c|}
\hline \multirow[b]{2}{*}{ Resource type } & \multirow[b]{2}{*}{ ID } & \multirow[b]{2}{*}{ Title, author } & \multirow[b]{2}{*}{ Yr } & \multicolumn{3}{|c|}{ Subtopics } \\
\hline & & & & B. $1^{\star}$ & B.2 & B. 3 \\
\hline \multirow[t]{9}{*}{$\begin{array}{l}\text { Web site } \\
\text { (Continued) }\end{array}$} & 88 & $\begin{array}{l}\text { European Agency for Safety and Health and Work } \\
\text { [Home page], European Agency for Safety and } \\
\text { Health and Work }\end{array}$ & 2005 & $\checkmark$ & & \\
\hline & 105 & Skin at work, HSE & 2005 & $\checkmark$ & $\checkmark$ & $\checkmark$ \\
\hline & 111 & $\begin{array}{l}\text { International Brotherhood of Teamsters [Home page], } \\
\text { International Brotherhood of Teamsters }\end{array}$ & 2006 & $\checkmark$ & & \\
\hline & 112 & ILO [Home page], ILO & 2005 & $\checkmark$ & $\checkmark$ & \\
\hline & 154 & $\begin{array}{l}\text { Toxicology Data Network(TOXNET)_Databases on } \\
\text { toxicology, hazardous chemicals, environmental } \\
\text { health, and toxic releases [Home page], National } \\
\text { Library of Medicine (NLM) }\end{array}$ & 2005 & $\checkmark$ & & \\
\hline & 162 & OSHA [Home page], OSHA & 2005 & $\checkmark$ & $\checkmark$ & $\checkmark$ \\
\hline & 165 & OR-OSHA [Home page], OR-OSHA & 2006 & $\checkmark$ & $\checkmark$ & \\
\hline & 174 & PCA [Home page], PCA & 2006 & & $\checkmark$ & \\
\hline & 207 & USACHPPM [Home page], USACHPPM & 2006 & $\checkmark$ & & $\checkmark$ \\
\hline
\end{tabular}

${ }^{\star}$ B.1=Job/Tasks, Industries/Processes, or Chemicals with Skin Exposures; B.2=Factors that Influence Exposure Conditions; B.3=Protocols/Checklists to Characterize Exposure to Skin Hazards

\section{Topic 3C. Hazard Identification}

Hazard identification is the process of establishing the existence of a hazard through the existence of hazards through field observations and laboratory analysis of the exposures and adverse health effects. This includes the identification of chemical substances that are harmful to the skin or harmful to the body if absorbed through the skin. As part of this process, the nature of the hazard is determined, such as causes to skin irritation, skin corrosion, skin sensitization, or some type of effect elsewhere in the body from absorption through the skin. The resources listed in this table will help in determining the kind of skin hazards from different chemicals. 


\section{Subtopic C.1. Risk Phrases, Hazard Symbols, Skin Designations}

These resources contain information on classifications of skin hazards associated with specific chemicals. Phrases, symbols, or other designations are used to describe the potential skin hazards. Skin hazards from chemicals can include (1) skin irritation and corrosion, (2) irritant contact dermatitis, (3) sensitization of skin and respiratory tract, (4) allergic contact dermatitis, (5) and contribution to overall body exposure.

\section{Subtopic C.2. Tables/Charts/Lists of Hazards for Specific Chemicals}

These resources include tables, charts, or lists of chemicals with the potential for significant skin exposures. These may include fact sheets that describe exposure conditions that may lead to harmful effects.

\section{Subtopic C.3. Protocols/Checklists to Identify Skin Hazards in the Workplace}

These resources provide protocols or checklists that can be used in the workplace to aid in the identification of skin hazards.

GENERAL AUDIENCE

Table 3C. Hazard identification

\begin{tabular}{|c|c|c|c|c|c|c|}
\hline \multirow[b]{2}{*}{ Resource type } & \multirow[b]{2}{*}{ ID } & \multirow[b]{2}{*}{ Title, author } & \multirow[b]{2}{*}{ Yr } & \multicolumn{3}{|c|}{ Subtopics } \\
\hline & & & & C. $1^{\star}$ & C.2 & C.3 \\
\hline $\begin{array}{l}\text { Book/monograph, } \\
\text { whole }\end{array}$ & 169 & $\begin{array}{l}\text { Essentials of occupational skin management, Pack- } \\
\text { ham CL }\end{array}$ & 1999 & & & $\checkmark$ \\
\hline Brochure, pamphlet & 7 & $\begin{array}{l}\text { Do you know about the health hazards of benzene? } \\
\text { Occupational Health Department }\end{array}$ & 2000 & $\checkmark$ & & \\
\hline \multirow[t]{2}{*}{$\begin{array}{l}\text { Brochure, pamphlet } \\
\text { (Continued) }\end{array}$} & 9 & $\begin{array}{l}\text { Assessing and managing risks at work from skin ex- } \\
\text { posure to chemical agents: Guidance for employers } \\
\text { and health and safety specialists, HSE }\end{array}$ & 2001 & & & $\checkmark$ \\
\hline & 95 & Quick selection guide to CPC, Forsberg K & 2002 & $\checkmark$ & $\checkmark$ & \\
\hline $\begin{array}{l}\text { Technical publica- } \\
\text { tion/report }\end{array}$ & 152 & NIOSH Pocket Guide to Chemical Hazards, NIOSH & 2004 & & $\checkmark$ & \\
\hline
\end{tabular}

(Continued) 


\section{GENERAL AUDIENCE Table 3C (Continued). Hazard identification}

\begin{tabular}{|c|c|c|c|c|c|c|}
\hline \multirow[b]{2}{*}{ Resource Type } & \multirow[b]{2}{*}{ ID } & \multirow[b]{2}{*}{ Title, Author } & \multirow[b]{2}{*}{ Yr } & \multicolumn{3}{|c|}{ Subtopics } \\
\hline & & & & C. $1^{*}$ & C.2 & C.3 \\
\hline \multirow[t]{2}{*}{ Web page } & 151 & $\begin{array}{l}\text { International Chemical Safety Cards (ISCS): U.S. } \\
\text { national version [Web site], NIOSH }\end{array}$ & 2005 & $\checkmark$ & $\checkmark$ & \\
\hline & 220 & $\begin{array}{l}\text { Dermatitis: Safety and health assessment and research } \\
\text { for prevention (SHARP) [Home page], WADLI }\end{array}$ & 2005 & & $\checkmark$ & \\
\hline \multirow[t]{10}{*}{ Web site } & 15 & $\begin{array}{l}\text { Occupational health and safety [Web site], } 1105 \\
\text { Media, Inc. }\end{array}$ & 2006 & $\checkmark$ & & $\checkmark$ \\
\hline & 22 & AAFP [Home page], AAFP & 2005 & & $\checkmark$ & \\
\hline & 58 & CCOHS [Home page], CCOHS & 2005 & & & $\checkmark$ \\
\hline & 59 & CPWR [Home page], CPWR & 2006 & & $\checkmark$ & \\
\hline & 63 & eLCOSH [Web site], CPWR & 2005 & & & $\checkmark$ \\
\hline & 88 & $\begin{array}{l}\text { European Agency for Safety and Health and Work } \\
\text { [Home page], European Agency for Safety and } \\
\text { Health and Work }\end{array}$ & 2005 & & $\checkmark$ & \\
\hline & 105 & Skin at work [Web site], HSE & 2005 & & & $\checkmark$ \\
\hline & 112 & ILO [Home page], ILO & 2005 & $\checkmark$ & $\checkmark$ & $\checkmark$ \\
\hline & 154 & $\begin{array}{l}\text { TOXNET-Databases on toxicology, hazardous } \\
\text { chemicals, environmental health, and toxic releases } \\
\text { [Home page], NLM }\end{array}$ & 2005 & $\checkmark$ & $\checkmark$ & \\
\hline & 162 & OSHA [Home page], OSHA & 2005 & $\checkmark$ & $\checkmark$ & $\checkmark$ \\
\hline
\end{tabular}

${ }^{\star}$ C.1 =Risk Phrases, Hazard Symbols, Skin Designations; C.2=Tables/Charts/Lists of Hazards for Specific Chemicals; C.3=Protocols/Checklists to Identify Skin Hazards in the Workplace 


\section{Topic 3D. Risk Assessment-Evaluating the Presence of Harmful Chemicals}

Risk Assessment is a measurement or an estimate of the chances of a given exposure to cause harm. With respect to skin exposures, risk assessments are performed by workplace health and safety representatives to provide the employer with some kind of estimate of the likelihood that an illness or injury will result from exposure of the skin to a particular chemical hazard. These resources provide guidance to identify whether a chemical skin exposure hazard is present in the workplace.

\section{Subtopic D.1. Protocols/Checklists to Identify Exposure Risk}

These resources provide protocols and checklists to be used to help individuals determine if an identified skin exposure hazard exists.

GENERAL AUDIENCE

Table 3D. Risk Assessment-Evaluating the presence of harmful chemicals

\begin{tabular}{|c|c|c|c|c|}
\hline & & & & Subtopics \\
\hline Resource type & ID & Title, author & Yr & D. $1^{\star}$ \\
\hline \multirow[t]{2}{*}{$\begin{array}{l}\text { Book/monograph, } \\
\text { whole }\end{array}$} & 169 & $\begin{array}{l}\text { Essentials of occupational skin management, Pack- } \\
\text { ham CL }\end{array}$ & 1999 & $\checkmark$ \\
\hline & 185 & Dermal exposure assessments, Sahmel J & 2006 & $\checkmark$ \\
\hline Brochure, pamphlet & 60 & $\begin{array}{l}\text { A safety and health practitioner's guide to skin protec- } \\
\text { tion, CPWR }\end{array}$ & 2000 & $\checkmark$ \\
\hline \multirow[t]{3}{*}{ Web site } & 63 & eLCOSH [Web site], CPWR & 2005 & $\checkmark$ \\
\hline & 105 & Skin at work [Web site], HSE & 2005 & $\checkmark$ \\
\hline & 162 & OSHA [Home page], OSHA & 2005 & $\checkmark$ \\
\hline
\end{tabular}

${ }^{\star}$ D. $1=$ Protocols/Checklists to Identify Exposure Risk 


\section{Topic 3E. Risk Management-Skin Exposure Risk Reduction}

Risk management is the process of controlling risks to workplace hazards. These resources provide basic information on how to (1) monitor for potential exposures and (2) determine what control options are available, and (3) how to decontaminate skin once exposure has occurred.

\section{Subtopic E.1. Overview of Skin Exposure Control Options}

These resources provide an overview on how to control skin exposures to chemicals. There are a number of different kinds of controls available to minimize exposures of the skin to harmful chemicals.

- The most effective control approach is to eliminate the use of the harmful chemical or substitute a less harmful chemical in its place. An example would be replacing a solvent-based cleaner that causes skin drying and irritation with a water-based cleaner.

- If elimination or substitution is not possible, the next favored method of control is an engineering control. An example of an engineering control is enclosing a process that releases vapors or dusts that are irritating to the skin. The enclosure would remove the potential for contact with the skin. Another common engineering control is the use of ventilation, where an exhaust fan draws the chemical vapor or dusts from the work area.

- If engineering controls cannot be put in place, then work practices should be changed. This means changing the way a worker performs a job or task in order to lower exposures. For example, if skin contact is occurring through contaminated work surfaces, then work surfaces should be cleaned regularly or covered with a disposable material that can be replaced regularly.

- Administrative controls can also be used. These include training programs (e.g., programs that show workers how to avoid skin contact), hazard monitoring, and medical surveillance programs to determine if workers are being exposed to harmful chemicals.

- When all else fails, the use of personal protective equipment (PPE), such as gloves and coveralls, can be used to lower exposures to workers. It is important to remember that PPE only works if it is properly selected, properly put on and worn, and properly taken off. This means that workers must be trained regularly, have an adequate size selection and supply of PPE, and be monitored regularly to ensure proper fit and use.

- Finally, employers can also implement skin management programs that promote good skin care. This can include creams that provide a protective 
barrier between the skin and the chemicals, as well as creams and lotions that remove chemicals from the skin and help maintain healthy skin.

\section{Subtopic E.2. Protocols/Checklists to Monitor Potential Exposures}

These resources contain protocols or checklists to use in routine qualitative monitoring of potential exposures to skin hazards. These will help identify whether chemical skin hazards are present in the workplace.

\section{Subtopic E.3. "Best practices"/Guidelines/Recommendations}

These resources include information on best practices, guidelines, or recommendations for chemical substitution, engineering controls, work practices, administrative controls, use of personal protective equipment, and implementation of a skin management program.

\section{Subtopic E.4. Guidelines/Recommendations for Post-exposure Skin Decontamination}

These resources contain information on how to decontaminate skin once skin exposures have occurred.

\section{GENERAL AUDIENCE}

Table 3E. Risk management-skin exposure risk reduction

\begin{tabular}{|c|c|c|c|c|c|c|c|}
\hline \multirow[b]{2}{*}{ Resource type } & \multirow[b]{2}{*}{ ID } & \multirow[b]{2}{*}{ Title, author } & \multirow[b]{2}{*}{ Yr } & \multicolumn{4}{|c|}{ Subtopics } \\
\hline & & & & E. $1^{\star}$ & E.2 & E.3 & E. 4 \\
\hline $\begin{array}{l}\text { Book/monograph, } \\
\text { whole }\end{array}$ & 169 & $\begin{array}{l}\text { Essentials of occupational skin management, } \\
\text { Packham CL }\end{array}$ & 1999 & $\checkmark$ & $\checkmark$ & $\checkmark$ & $\checkmark$ \\
\hline \multirow[t]{3}{*}{ Brochure, pamphlet } & 6 & $\begin{array}{l}\text { Selecting protective gloves for work with } \\
\text { chemicals, HSE }\end{array}$ & 2000 & & & $\checkmark$ & \\
\hline & 7 & $\begin{array}{l}\text { Do you know about the health hazards of } \\
\text { benzene?, Occupational Health Department }\end{array}$ & 2000 & & & $\checkmark$ & \\
\hline & 8 & $\begin{array}{l}\text { Did you know the hazards of solvents?, } \\
\text { Occupational Health Department }\end{array}$ & 2000 & & & $\checkmark$ & \\
\hline
\end{tabular}

\footnotetext{
*See footnotes at end of table.
} 
GENERAL AUDIENCE

Table 3E (Continued). Risk management—Skin Exposure Risk Reduction

\begin{tabular}{|c|c|c|c|c|c|c|c|}
\hline \multirow[b]{2}{*}{ Resource type } & \multirow[b]{2}{*}{ ID } & \multirow[b]{2}{*}{ Title, author } & \multirow[b]{2}{*}{ Yr } & \multicolumn{4}{|c|}{ Subtopics } \\
\hline & & & & E. $1^{\star}$ & E. 2 & E. 3 & E.4 \\
\hline \multirow[t]{6}{*}{$\begin{array}{l}\text { Brochure, pamphlet } \\
\text { (Continued) }\end{array}$} & 9 & $\begin{array}{l}\text { Assessing and managing risks at work from } \\
\text { skin exposure to chemical agents: Guidance } \\
\text { for employers and health and safety special- } \\
\text { ists, HSE }\end{array}$ & 2001 & & & $\checkmark$ & \\
\hline & 11 & $\begin{array}{l}\text { Cost and effectiveness of chemical protective } \\
\text { gloves for the workplace, HSE }\end{array}$ & 2001 & & & $\checkmark$ & \\
\hline & 12 & $\begin{array}{l}\text { Choice of skin care products for the work- } \\
\text { place, HSE }\end{array}$ & 2001 & & & $\checkmark$ & \\
\hline & 60 & $\begin{array}{l}\text { A safety and health practitioner's guide to } \\
\text { skin protection, CPWR }\end{array}$ & 2000 & $\checkmark$ & $\checkmark$ & $\checkmark$ & $\checkmark$ \\
\hline & 62 & $\begin{array}{l}\text { An employer's guide to skin protection, } \\
\text { CPWR }\end{array}$ & 2005 & $\checkmark$ & & $\checkmark$ & $\checkmark$ \\
\hline & 95 & Quick selection guide to CPC, Forsberg K & 2002 & & & $\checkmark$ & \\
\hline Guideline & 3 & $\begin{array}{c}\text { What you need to know about occupational } \\
\text { exposure to metalworking fluids, NIOSH }\end{array}$ & 1998 & & & $\checkmark$ & \\
\hline \multirow[t]{2}{*}{$\begin{array}{l}\text { Journal article- } \\
\text { review, meta- } \\
\text { analysis }\end{array}$} & 145 & $\begin{array}{l}\text { Systemic toxicity from skin exposures (or } \\
\text { what happens when you do not decontami- } \\
\text { nate), McDougal JN }\end{array}$ & 2007 & & & & $\checkmark$ \\
\hline & 147 & $\begin{array}{l}\text { The value and limitations of protective gloves } \\
\text { in medical health service: Part III, Mell- } \\
\text { strom GA }\end{array}$ & 1996 & & & $\checkmark$ & \\
\hline \multirow[t]{3}{*}{ Magazine article } & 52 & Chemical hand protection, Brown JW & 2002 & & & $\checkmark$ & \\
\hline & 72 & $\begin{array}{l}\text { Helping hands. Skin care for the hands, } \\
\text { Crassweller I }\end{array}$ & 1999 & & & $\checkmark$ & $\checkmark$ \\
\hline & 75 & $\begin{array}{l}\text { Protecting the hand-skin barrier in the work- } \\
\text { place, Del Rosso, J }\end{array}$ & 2001 & & & $\checkmark$ & \\
\hline
\end{tabular}

*See footnotes at end of table. 
GENERAL AUDIENCE

Table 3E (Continued). Risk management-Skin Exposure Risk Reduction

\begin{tabular}{|c|c|c|c|c|c|c|c|}
\hline \multirow[b]{2}{*}{ Resource type } & \multirow[b]{2}{*}{ ID } & \multirow[b]{2}{*}{ Title, author } & \multirow[b]{2}{*}{ Yr } & \multicolumn{4}{|c|}{ Subtopics } \\
\hline & & & & E. $1^{\star}$ & E.2 & E.3 & E.4 \\
\hline \multirow{3}{*}{$\begin{array}{l}\text { Magazine article } \\
\text { (Continued) }\end{array}$} & 149 & Skin care: Starting from scratch, Nash JL & 2000 & $\checkmark$ & & $\checkmark$ & \\
\hline & 181 & $\begin{array}{l}\text { Safe use of glutaraldehyde, Romano- } \\
\text { Woodward D }\end{array}$ & 2000 & & $\checkmark$ & $\checkmark$ & $\checkmark$ \\
\hline & 186 & $\begin{array}{l}\text { Protecting hands against chemical exposures, } \\
\text { Sarkis K }\end{array}$ & 2000 & & & $\checkmark$ & \\
\hline $\begin{array}{l}\text { Other-Guideline } \\
\text { from private lab }\end{array}$ & 70 & $\begin{array}{l}\text { A guide to dermal exposure reduction, Color- } \\
\text { metric Laboratories Inc. }\end{array}$ & 1999 & $\checkmark$ & & & \\
\hline $\begin{array}{l}\text { Technical publica- } \\
\text { tion/report }\end{array}$ & 152 & $\begin{array}{l}\text { NIOSH pocket guide to chemical hazards, } \\
\text { NIOSH }\end{array}$ & 2004 & & & $\checkmark$ & $\checkmark$ \\
\hline \multirow[t]{5}{*}{ Web page } & 21 & $\begin{array}{l}\text { Skin problems: How to protect yourself from } \\
\text { job-related skin problems [Web site], AAFP }\end{array}$ & 2004 & $\checkmark$ & & & \\
\hline & 150 & $\begin{array}{l}\text { Recommendations for CPC: A companion } \\
\text { to the NIOSH pocket guide to chemical } \\
\text { hazards [Web site], NIOSH }\end{array}$ & 2005 & & & $\checkmark$ & \\
\hline & 151 & ISCS: U.S. national version [Web site], NIOSH & 2005 & $\checkmark$ & & $\checkmark$ & $\checkmark$ \\
\hline & 205 & Health and safety zone [Home page], Unison & 2005 & $\checkmark$ & & & \\
\hline & 220 & $\begin{array}{l}\text { Dermatitis: safety and health assessment and } \\
\text { research for prevention (SHARP) [Home } \\
\text { page] WADLI }\end{array}$ & 2005 & $\checkmark$ & & $\checkmark$ & \\
\hline \multirow[t]{4}{*}{ Web site } & 14 & $\begin{array}{l}\text { MT DLI-Employment Relations [Home } \\
\text { page], MT DLI }\end{array}$ & 2005 & $\sqrt{ }$ & & $\sqrt{ }$ & $\checkmark$ \\
\hline & 15 & $\begin{array}{l}\text { Occupational safety and health [Web site], } \\
1105 \text { Media, Inc. }\end{array}$ & 2006 & & $\checkmark$ & $\checkmark$ & \\
\hline & 16 & CPI, [Web site], ACC & 2006 & $\checkmark$ & & $\checkmark$ & $\checkmark$ \\
\hline & 22 & AAFP [Home page], AAFP & 2005 & $\checkmark$ & & $\checkmark$ & \\
\hline
\end{tabular}

*See footnotes at end of table.

(Continued) 
GENERAL AUDIENCE

Table 3E (Continued). Risk management—Skin Exposure Risk Reduction

\begin{tabular}{|c|c|c|c|c|c|c|c|}
\hline \multirow[b]{2}{*}{ Resource type } & \multirow[b]{2}{*}{ ID } & \multirow[b]{2}{*}{ Title, author } & \multirow[b]{2}{*}{ Yr } & \multicolumn{4}{|c|}{ Subtopics } \\
\hline & & & & E. $1^{\star}$ & E. 2 & E.3 & E.4 \\
\hline \multirow[t]{14}{*}{$\begin{array}{l}\text { Web site } \\
\text { (Continued) }\end{array}$} & 29 & $\begin{array}{l}\text { Ansell Chemsafe [Home page], Ansell Chem- } \\
\text { safe }\end{array}$ & 2005 & $\sqrt{ }$ & & $\checkmark$ & \\
\hline & 58 & CCOHS [Home page], CCOHS & 2005 & & & $\checkmark$ & \\
\hline & 59 & CPWR [Home page], CPWR & 2006 & $\checkmark$ & & $\checkmark$ & $\checkmark$ \\
\hline & 63 & eLCOSH [Web site], CPWR & 2005 & & & $\checkmark$ & $\checkmark$ \\
\hline & 64 & $\begin{array}{l}\text { National Ag Safety Database (NASD) [Web } \\
\text { site], Centers for Disease Control and Pre- } \\
\text { vention }[\mathrm{CDC}]\end{array}$ & 2006 & $\sqrt{ }$ & & $\checkmark$ & $\checkmark$ \\
\hline & 88 & $\begin{array}{l}\text { European Agency for Safety and Health and } \\
\text { Work [Home page], European Agency for } \\
\text { Safety and Health and Work }\end{array}$ & 2005 & & & $\checkmark$ & \\
\hline & 105 & Skin at work [Web site], HSE & 2005 & $\checkmark$ & $\checkmark$ & $\checkmark$ & \\
\hline & 111 & $\begin{array}{l}\text { International Brotherhood of Teamsters } \\
\text { [Home page], International Brotherhood of } \\
\text { Teamsters }\end{array}$ & 2006 & $\checkmark$ & & & \\
\hline & 112 & ILO [Home page], ILO & 2005 & $\checkmark$ & & $\checkmark$ & $\checkmark$ \\
\hline & 154 & $\begin{array}{l}\text { TOXNET_Databases on toxicology, hazard- } \\
\text { ous chemicals, environmental health, and } \\
\text { toxic releases [Home page], NLM }\end{array}$ & 2005 & $\checkmark$ & & & $\checkmark$ \\
\hline & 156 & $\begin{array}{l}\text { National Ready Mixed Concrete Association } \\
\text { [Home page], NRMCA }\end{array}$ & 2006 & $\checkmark$ & & $\checkmark$ & \\
\hline & 162 & OSHA [Home page], OSHA & 2005 & $\checkmark$ & $\checkmark$ & $\checkmark$ & $\checkmark$ \\
\hline & 165 & OR-OSHA [Home page], OR-OSHA & 2006 & $\checkmark$ & & $\checkmark$ & \\
\hline & 174 & PCA [Home page], PCA & 2006 & $\checkmark$ & & $\checkmark$ & \\
\hline
\end{tabular}

${ }^{\star}$ See footnotes at end of table.

(Continued) 
GENERAL AUDIENCE

Table 3E (Continued). Risk management—Skin Exposure Risk Reduction

\begin{tabular}{|c|c|c|c|c|c|c|c|}
\hline \multirow[b]{2}{*}{ Resource type } & \multirow[b]{2}{*}{ ID } & \multirow[b]{2}{*}{ Title, author } & \multirow[b]{2}{*}{ Yr } & \multicolumn{4}{|c|}{ Subtopics } \\
\hline & & & & E. $1^{\star}$ & E.2 & E.3 & E.4 \\
\hline \multirow{2}{*}{$\begin{array}{l}\text { Web site } \\
\quad \text { (Continued) }\end{array}$} & 207 & USACHPPM [Home page], USACHPPM & 2006 & & & $\checkmark$ & $\checkmark$ \\
\hline & 225 & $\begin{array}{l}\text { International Programme on Chemical Safety } \\
\text { (IPCS) [Home page], WHO }\end{array}$ & 2005 & & & & $\checkmark$ \\
\hline
\end{tabular}

${ }^{\star}$ E.1=Overview of Skin Exposure Control Options; E.2=Protocols/Checklists to Monitor Potential Exposures;

E.3="Best Practices"/Guidelines/Recommendations; E.4=Guidelines/Recommendations for Postexposure Skin Decontamination 


\section{CHAPTER 4 \\ Resources for the Professional Audience}

\subsection{Introduction}

The resources identified below are appropriate for a professional audience to use in investigating and controlling harmful skin exposures in the workplace. Included in the professional audience are those who may be involved in conducting scientific risk assessments and preparing technical recommendations. In general, they have adequate knowledge to use technical information for evaluating, recognizing, and controlling harmful skin exposures. The professional audience may include industrial hygienists, occupational epidemiologists, dermatologists, occupational physicians and nurses, academic researchers and policy makers.

Professional audience members who are looking for more general treatments of some of the topics provided below, including material appropriate for worker training activities, are encouraged to also look at the resources identified for the general audience in Chapter 3.

As a reminder, the resources are review articles published in peer reviewed journals, as well as books, magazines, Web sites, regulatory guidelines, databases, brochures and a number of other types of resources. Also, they are not meant to be a comprehensive list of review information available for the professional audience, but rather a representative list of what is available. In addition, the accuracy of the information contained in any resource has not been evaluated.

\subsection{Resources for the Professional Audience by Topic}

The following tables provide a review of resources covering each of six major topics, as listed below, related to occupational skin exposure to chemicals for a professional audience. The major topics are further divided into subtopics, each of which is represented in the columns on the right-hand side of the tables. Descriptions of each topic and its related subtopics are given before each table to assist users in deciding what kind of information they are interested in obtaining.

For the professional audience, the six major topics and related subtopics are:

Topic 4A. Overview of the Investigation and Control of Occupational Skin Exposures 
A.1. Occurrence of Skin Exposures in the Workplace

A.2. Health Hazards Resulting from Skin Exposure to Chemicals

A.3. Investigation, Intervention, and Control of Occupational Skin Exposures

A.4. Skin Physiology and Function as Barriers to Chemical Insults

A.5. Dermal Regulations and Skin Notations

Topic 4B. Surveillance and Clinical Aspects

B.1. Surveillance Studies Reporting Incidences of Occupational Skin Exposures

B.2. Loss of Workdays and Impact on Productivity

B.3. Surveillance Study Protocols/Procedures for Gathering Data

B.4. Clinical Protocols for Recognition of Skin Exposure Health Effects

Topic 4C. Exposure Characterization

C.1. Workplace Factors Associated with Harmful Skin Exposures

C.2. Description of Factors Influencing Exposure Conditions

C.3. Checklists/Questionnaires to Quantify Skin Exposure Incidents

C4. Methods to Measure Exposures

C.5. Exposure Modeling

Topic 4D. Hazard Identification from Toxicological Studies or Modeling

D.1. Potential Health Effects Resulting from Specific Chemicals

D.2. Summaries of Health Effects and Dose-Response Relationships

D.3. Characterization Protocols

Topic 4E. Risk Assessment

E.1. Guidelines for Risk Assessment or Analysis

E.2. Example of Risk Assessment

Topic 4F. Risk Management

F. 1. Exposure Control Strategies

F.2. Risk Assessment Protocols 
As in Chapter 3, each of the tables presented here include the following columns:

- Resource Type-whether the resource is a book, brochure, journal article, Web site, and so forth.

- ID-unique ID number assigned to each resource, can be used to locate each resource in Appendix A, organized in alphabetical/numerical order by resource type.

- Title, Author - the resource title and author, if listed, for each resource.

- $\mathrm{Yr}$ - the publication year of the resource and, in the case of Web sites and Web pages, the year the Web site or Web page was reviewed for inclusion in the Indexed Dermal Bibliography.

- Subtopics-Subtopics addressed under the given table topic. The definition of each subtopic is listed at the bottom of each table. Each subtopic addressed by a resource is checked.

A given resource may be repeated in multiple tables. This will happen when a resource provides information covering a variety topic areas.

\section{Topic 4A. Overview of the Investigation and Control of Occupational Skin Exposures}

These resources provide an overview of the occurrence of skin exposures to chemicals in the workplace; health hazards that can result from skin exposure to chemicals; the process of investigating, intervening and controlling occupational skin exposures; and background information on basic skin physiology and how skin functions as a barrier to chemical absorption into the body.

\section{Subtopic A.1. Occurrence of Skin Exposures in the Workplace}

These resources provide a general overview of the occurrence of skin exposures to harmful chemicals in the workplace. This overview may not be the primary focus of the resource, but rather introductory material that leads into the main focus of the resource.

\section{Subtopic A.2. Health Hazards Resulting from Skin Exposure to Chemicals}

These resources provide an overview description of the different kinds of adverse health effects that can result from skin exposure to chemicals. 


\section{Subtopic A.3. Investigation, Intervention, and Control of Occupa- tional Skin Exposures}

These resources provide an introduction to the recognition, evaluation and control of skin hazards in the workplace. Background information can be found on skin hazards, including an overview of occupational skin exposure investigations, intervention approaches that can be adopted and control strategies that can be put into place.

\section{Subtopic A.4. Skin Physiology and Function as a Barrier to Chemi- cal Insults}

These resources provide an introduction to skin physiology, including the function of the different layers of skin, how they act as barries to chemical absorption, and how they can alter the skin's natural barrier properties when compromised or damaged.

\section{Subtopic A.5. Dermal Regulations and Skin Notations}

These resources either contain information about regulations covering occupational skin exposure to chemicals or information about the designation of chemicals based on their skin hazard or their potential to be absorbed by the skin. The governmental Web sites listed contain information on applicable standards. In addition to regulatory information, some of these resources also contain information on chemicals with skin notations.

PROFESSIONAL AUDIENCE

Table 4A. Overview of the investigation and control of occupational skin exposures

Subtopics

\begin{tabular}{|c|c|c|c|c|c|c|c|c|}
\hline \multirow[b]{2}{*}{ Resource type } & \multirow[b]{2}{*}{ ID } & \multirow[b]{2}{*}{ Title, author } & \multirow[b]{2}{*}{ Yr } & \\
\hline & & & & A. $1^{\star}$ & A. 2 & A. 3 & A. 4 & A. 5 \\
\hline \multirow[t]{2}{*}{$\begin{array}{l}\text { Book/monograph, } \\
\text { chapter }\end{array}$} & 4 & $\begin{array}{l}\text { Occupational skin exposure-absorption } \\
\text { of chemical agents and assessment of } \\
\text { exposures, Harris R }\end{array}$ & 2000 & & & & $\checkmark$ & \\
\hline & 28 & $\begin{array}{l}\text { Systemic toxicity from percutaneous } \\
\text { absorption, Andersen KE }\end{array}$ & 1999 & & & & $\checkmark$ & \\
\hline
\end{tabular}

\footnotetext{
*See footnotes at end of table.
} 
PROFESSIONAL AUDIENCE

Table 4A (Continued). Overview of the investigation and control of occupational skin exposures

\begin{tabular}{|c|c|c|c|c|c|c|c|c|}
\hline \multirow[b]{2}{*}{ Resource type } & \multirow[b]{2}{*}{ ID } & \multirow[b]{2}{*}{ Title, author } & \multirow[b]{2}{*}{ Yr } & \multicolumn{5}{|c|}{ Subtopics } \\
\hline & & & & A. $1^{\star}$ & A.2 & A.3 & A.4 & A.5 \\
\hline \multirow[t]{7}{*}{$\begin{array}{l}\text { Book/monograph, } \\
\text { chapter }\end{array}$} & 40 & $\begin{array}{l}\mathrm{CPC} \text { and the skin: Practical consider- } \\
\text { ations, Boeniger M }\end{array}$ & 2002 & & & & $\checkmark$ & \\
\hline & 45 & $\begin{array}{l}\text { Protective gloves for occupational use, } \\
\text { Boman A }\end{array}$ & 2005 & & & & & $\checkmark$ \\
\hline & 157 & $\begin{array}{l}\text { Surface and dermal monitoring for } \\
\text { toxic exposures, Ness SA }\end{array}$ & 1994 & & $\checkmark$ & $\checkmark$ & & \\
\hline & 185 & Dermal exposure assessments, Sahmel J & 2006 & & & & $\checkmark$ & \\
\hline & 218 & $\begin{array}{l}\text { Health risk assessment: Dermal and } \\
\text { inhalation exposure and absorption of } \\
\text { toxicants (dermatology), Wang RGM }\end{array}$ & 1993 & & & & $\checkmark$ & \\
\hline & 221 & $\begin{array}{l}\text { Development of occupational skin dis- } \\
\text { ease, Weber LW }\end{array}$ & 2003 & & & & $\checkmark$ & \\
\hline & 229 & Dermatotoxicology, Zhai $\mathrm{H}$ & 2004 & & & & $\checkmark$ & \\
\hline \multirow[t]{2}{*}{$\begin{array}{l}\text { Brochure, pam- } \\
\text { phlet }\end{array}$} & 60 & $\begin{array}{l}\text { A safety and health practitioner's guide } \\
\text { to skin protection, CPWR }\end{array}$ & 2000 & $\checkmark$ & $\checkmark$ & & $\checkmark$ & \\
\hline & 61 & $\begin{array}{l}\text { Physician's alert for occupational con- } \\
\text { tact dermatitis among construction } \\
\text { workers, CPWR }\end{array}$ & 2001 & $\checkmark$ & $\checkmark$ & & & \\
\hline \multirow[t]{2}{*}{ Guideline } & 47 & $\begin{array}{l}\text { Guideline for hand hygiene in health- } \\
\text { care settings, CDC }\end{array}$ & 2002 & & & & $\checkmark$ & \\
\hline & 161 & $\begin{array}{l}\text { Sampling for surface contamination, } \\
\text { OSHA }\end{array}$ & 2005 & & & & & $\checkmark$ \\
\hline $\begin{array}{l}\text { Journal article- } \\
\text { primary }\end{array}$ & 37 & $\begin{array}{l}\text { Slow curing of aliphatic polyisocyanate } \\
\text { paints in automotive refinishing: A po- } \\
\text { tential source for skin exposure, Bello D }\end{array}$ & 2007 & $\checkmark$ & & & & \\
\hline
\end{tabular}

${ }^{\star}$ See footnotes at end of table.

(Continued) 
PROFESSIONAL AUDIENCE

\section{Table 4A (Continued). Overview of the investigation and control of occupational skin exposures}

\begin{tabular}{|c|c|c|c|c|c|c|c|c|}
\hline \multirow[b]{2}{*}{ Resource type } & \multirow[b]{2}{*}{ ID } & \multirow[b]{2}{*}{ Title, author } & \multirow[b]{2}{*}{ Yr } & \multicolumn{5}{|c|}{ Subtopics } \\
\hline & & & & A. $1^{*}$ & A. 2 & A. 3 & A.4 & A. 5 \\
\hline \multirow[t]{4}{*}{$\begin{array}{l}\text { Journal article- } \\
\text { primary }\end{array}$} & 68 & $\begin{array}{l}\text { Surveillance of occupational skin dis- } \\
\text { ease: EPIDERM and OPRA, Cherry N }\end{array}$ & 2000 & $\checkmark$ & & & & \\
\hline & 123 & $\begin{array}{l}\text { Skin cleansers for occupational use: } \\
\text { testing the skin compatibility of differ- } \\
\text { ent formulations, Klotz A }\end{array}$ & 2003 & & & & $\checkmark$ & \\
\hline & 131 & $\begin{array}{l}\text { Skin exposure to aliphatic polyiso- } \\
\text { cyanates in the auto body repair and } \\
\text { refinishing industry: A qualitative } \\
\text { assessment, Liu Y }\end{array}$ & 2007 & $\checkmark$ & & & & \\
\hline & 142 & $\begin{array}{l}\text { Dermal exposure and urinary } \\
\text { 1-hydroxypyrene among asphalt roof- } \\
\text { ing workers, McClean MD }\end{array}$ & 2007 & $\checkmark$ & & & & \\
\hline \multirow[t]{5}{*}{$\begin{array}{l}\text { Journal article- } \\
\text { review, meta- } \\
\text { analysis }\end{array}$} & 1 & $\begin{array}{l}\text { Skin lesions and environmental expo- } \\
\text { sures. An overview for the occupa- } \\
\text { tional health nurse, ATSDR }\end{array}$ & 1996 & & $\checkmark$ & & $\checkmark$ & \\
\hline & 30 & $\begin{array}{l}\text { Occupational contact dermatitis, } \\
\text { Antezana, } \mathrm{M}\end{array}$ & 2003 & & $\checkmark$ & & & \\
\hline & 34 & $\begin{array}{l}\text { Classification criteria for skin-sensitizing } \\
\text { chemicals: A commentary, Basketter } \\
\text { DA }\end{array}$ & 1999 & & & $\checkmark$ & & $\checkmark$ \\
\hline & 35 & $\begin{array}{l}\text { Factors affecting thresholds in allergic } \\
\text { contact dermatitis: Safety and regula- } \\
\text { tory considerations, Basketter DA }\end{array}$ & 2002 & & & & $\checkmark$ & \\
\hline & 38 & $\begin{array}{l}\text { Skin exposure to isocyanates: Reasons } \\
\text { for concern, Bello D }\end{array}$ & 2007 & $\checkmark$ & & & & \\
\hline
\end{tabular}

*See footnotes at end of table.

(Continued) 
PROFESSIONAL AUDIENCE

Table 4A (Continued). Overview of the investigation and control of occupational skin exposures

\begin{tabular}{|c|c|c|c|c|c|c|c|c|}
\hline \multirow[b]{2}{*}{ Resource type } & \multirow[b]{2}{*}{ ID } & \multirow[b]{2}{*}{ Title, author } & \multirow[b]{2}{*}{ Yr } & \multicolumn{5}{|c|}{ Subtopics } \\
\hline & & & & A. $1^{*}$ & A. 2 & A. 3 & A.4 & A. 5 \\
\hline \multirow[t]{8}{*}{$\begin{array}{l}\text { Journal article- } \\
\text { review, meta- } \\
\text { analysis } \\
\text { (Continued) }\end{array}$} & 46 & $\begin{array}{l}\text { Proposal for the assessment of quan- } \\
\text { titative dermal exposure limits in } \\
\text { occupational environments: Part } 1 . \\
\text { Development of a concept to derive } \\
\text { a quantitative dermal occupational } \\
\text { exposure limit, Bos PM }\end{array}$ & 1998 & & & & & $\checkmark$ \\
\hline & 51 & $\begin{array}{l}\text { Concepts of skin protection: Consider- } \\
\text { ations for the evaluation and termi- } \\
\text { nology of the performance of skin } \\
\text { protective equipment, Brouwer DH }\end{array}$ & 2005 & & & $\checkmark$ & $\checkmark$ & \\
\hline & 69 & $\begin{array}{l}\text { Occupational issues of irritant contact } \\
\text { dermatitis, Chew AL }\end{array}$ & 2003 & & $\checkmark$ & & & \\
\hline & 73 & $\begin{array}{l}\text { Pesticide-related illness among migrant } \\
\text { farm workers in the United States, } \\
\text { Das R }\end{array}$ & 2001 & & $\checkmark$ & & & \\
\hline & 78 & $\begin{array}{l}\text { The epidemiology of occupational con- } \\
\text { tact dermatitis, Diepgen TL }\end{array}$ & 1999 & & $\checkmark$ & & & \\
\hline & 79 & $\begin{array}{l}\text { Skin-conditioning products in occupa- } \\
\text { tional dermatology, Elsner P }\end{array}$ & 2003 & & & & $\checkmark$ & \\
\hline & 81 & $\begin{array}{l}\text { Occupational contact dermatitis II: Risk } \\
\text { assessment and prognosis, Emmett } \\
\text { EA }\end{array}$ & 2003 & & & $\checkmark$ & $\checkmark$ & \\
\hline & 83 & $\begin{array}{l}\text { Detailed review document on classifica- } \\
\text { tion systems for skin irritation/cor- } \\
\text { rosion in OECD member countries, } \\
\text { OECD }\end{array}$ & 1999 & & & & & $\checkmark$ \\
\hline
\end{tabular}

*See footnotes at end of table.

(Continued) 
PROFESSIONAL AUDIENCE

\section{Table 4A (Continued). Overview of the investigation and control of} occupational skin exposures

\begin{tabular}{|c|c|c|c|c|c|c|c|c|}
\hline \multirow[b]{2}{*}{ Resource type } & \multirow[b]{2}{*}{ ID } & \multirow[b]{2}{*}{ Title, author } & \multirow[b]{2}{*}{ Yr } & \multicolumn{5}{|c|}{ Subtopics } \\
\hline & & & & A. $1^{*}$ & A. 2 & A.3 & A. 4 & A. 5 \\
\hline \multirow[t]{10}{*}{$\begin{array}{l}\text { Journal article- } \\
\text { review, meta- } \\
\text { analysis } \\
\text { (Continued) }\end{array}$} & 97 & $\begin{array}{l}\text { Developing control of substances haz- } \\
\text { ardous to health (COSHH) regula- } \\
\text { tions essentials: Dermal exposure, } \\
\text { personal protective equipment and } \\
\text { first aid, Garrod AN }\end{array}$ & 2003 & & & & & $\checkmark$ \\
\hline & 101 & $\begin{array}{l}\text { CCP and workplace safety: A review, } \\
\text { Graves CG }\end{array}$ & 2000 & $\checkmark$ & $\checkmark$ & & & \\
\hline & 103 & $\begin{array}{l}\text { Occupation-related allergies in den- } \\
\text { tistry, Hamann CP }\end{array}$ & 2005 & $\checkmark$ & $\checkmark$ & & & \\
\hline & 106 & $\begin{array}{l}\text { Misinterpretation and misuse of expo- } \\
\text { sure limits, Hewett } \mathrm{P}\end{array}$ & 2001 & & & & & $\checkmark$ \\
\hline & 115 & $\begin{array}{l}\text { Dermal absorption of benzene: Implica- } \\
\text { tions for work practices and regula- } \\
\text { tions, Kalnas J }\end{array}$ & 2000 & $\checkmark$ & & & & $\checkmark$ \\
\hline & 118 & $\begin{array}{l}\text { The role of the skin in the development } \\
\text { of chemical respiratory hypersensitiv- } \\
\text { ity, Kimber I }\end{array}$ & 1996 & & $\checkmark$ & & & \\
\hline & 122 & $\begin{array}{l}\text { A critique of assumptions about select- } \\
\text { ing chemical-resistant gloves: A case } \\
\text { for workplace evaluation of glove } \\
\text { efficacy, Klingner TD }\end{array}$ & 2002 & & & & & $\checkmark$ \\
\hline & 124 & $\begin{array}{l}\text { Occupational contact dermatitis. Rec- } \\
\text { ognition and management, Koch P }\end{array}$ & 2001 & & $\checkmark$ & & & \\
\hline & 134 & $\begin{array}{l}\text { The importance of occupational skin } \\
\text { diseases in the United States, Lushniak } \\
\text { BD }\end{array}$ & 2003 & $\checkmark$ & & & & \\
\hline & 135 & $\begin{array}{l}\text { Occupational contact dermatitis, Lush- } \\
\text { niak BD }\end{array}$ & 2004 & $\checkmark$ & $\checkmark$ & $\checkmark$ & & \\
\hline
\end{tabular}

*See footnotes at end of table.

(Continued) 
PROFESSIONAL AUDIENCE

Table 4A (Continued). Overview of the investigation and control of occupational skin exposures

\begin{tabular}{|c|c|c|c|c|c|c|c|c|}
\hline \multirow[b]{2}{*}{ Resource type } & \multirow[b]{2}{*}{ ID } & \multirow[b]{2}{*}{ Title, author } & \multirow[b]{2}{*}{ Yr } & \multicolumn{5}{|c|}{ Subtopics } \\
\hline & & & & A. $1^{\star}$ & A. 2 & A.3 & A.4 & A.5 \\
\hline \multirow{11}{*}{$\begin{array}{l}\text { Journal article- } \\
\text { review, meta- } \\
\text { analysis } \\
\text { (Continued) }\end{array}$} & 138 & $\begin{array}{l}\text { Harmonization of future needs for der- } \\
\text { mal exposure assessment and model- } \\
\text { ing: a workshop report, Marquart } \mathrm{H}\end{array}$ & 2001 & & & & & $\checkmark$ \\
\hline & 140 & $\begin{array}{l}\text { Dermal toxicity due to industrial } \\
\text { chemicals, Mathur AK }\end{array}$ & 2002 & & & & $\checkmark$ & \\
\hline & 144 & $\begin{array}{l}\text { Methods for assessing risks of dermal ex- } \\
\text { posures in the workplace, McDougal JN }\end{array}$ & 2002 & & & $\checkmark$ & $\checkmark$ & \\
\hline & 159 & $\begin{array}{l}\text { Criteria for skin notation in different } \\
\text { countries, Nielsen JB }\end{array}$ & 2004 & & & & & $\checkmark$ \\
\hline & 183 & Solvents and the skin, Rowse DH & 2004 & & $\checkmark$ & & $\checkmark$ & $\checkmark$ \\
\hline & 187 & $\begin{array}{l}\text { Percutaneous penetration studies for } \\
\text { risk assessment, Sartorelli P }\end{array}$ & 2000 & & & $\checkmark$ & & \\
\hline & 188 & $\begin{array}{l}\text { Dermal exposure assessment in occupa- } \\
\text { tional medicine, Sartorelli P }\end{array}$ & 2002 & $\checkmark$ & & & & \\
\hline & 192 & $\begin{array}{l}\text { When should a substance be designated } \\
\text { as sensitizing for the skin ('Sh') or for } \\
\text { the airways ('Sa')? Schnuch A }\end{array}$ & 2002 & & & & & $\checkmark$ \\
\hline & 194 & $\begin{array}{l}\text { Dermal exposure to chemicals in the } \\
\text { workplace: Just how important is skin } \\
\text { absorption? Semple S }\end{array}$ & 2004 & $\checkmark$ & $\checkmark$ & & $\checkmark$ & \\
\hline & 197 & $\begin{array}{l}\text { From xenobiotic chemistry and me- } \\
\text { tabolism to better prediction and } \\
\text { risk assessment of skin allergy, Smith } \\
\text { Pease CK }\end{array}$ & 2003 & & & & $\checkmark$ & \\
\hline & 203 & $\begin{array}{l}\text { Management of dermatitis in the rubber } \\
\text { manufacturing industry, Toeppen- } \\
\text { Sprigg B }\end{array}$ & 1999 & $\checkmark$ & $\checkmark$ & $\checkmark$ & & \\
\hline
\end{tabular}

*See footnotes at end of table.

(Continued) 
PROFESSIONAL AUDIENCE

Table 4A (Continued). Overview of the investigation and control of occupational skin exposures

\begin{tabular}{|c|c|c|c|c|c|c|c|c|}
\hline \multirow[b]{2}{*}{ Resource type } & \multirow[b]{2}{*}{ ID } & \multirow[b]{2}{*}{ Title, author } & \multirow[b]{2}{*}{ Yr } & \multicolumn{5}{|c|}{ Subtopics } \\
\hline & & & & A. $1^{\star}$ & A. 2 & A.3 & A. 4 & A. 5 \\
\hline \multirow{3}{*}{$\begin{array}{l}\text { Journal article- } \\
\text { review, meta- } \\
\text { analysis } \\
\text { (Continued) }\end{array}$} & 204 & $\begin{array}{l}\text { Prediction of irritancy in the human } \\
\text { skin irritancy model and occupational } \\
\text { setting, Tupker RA }\end{array}$ & 2003 & & & & $\checkmark$ & \\
\hline & 210 & $\begin{array}{l}\text { From dermal exposure to internal dose, } \\
\text { van de Sandt J }\end{array}$ & 2008 & & & & $\checkmark$ & \\
\hline & 213 & $\begin{array}{l}\text { Review of skin permeation hazard of } \\
\text { bitumen fumes, van Rooij JG }\end{array}$ & 2008 & $\checkmark$ & $\checkmark$ & $\checkmark$ & $\checkmark$ & \\
\hline $\begin{array}{l}\text { Other- } \\
\text { commentary }\end{array}$ & 41 & $\begin{array}{l}\text { Exposure and absorption of hazardous } \\
\text { materials through the skin, Boeniger } \\
\text { MF }\end{array}$ & 2000 & & & & & $\checkmark$ \\
\hline Other-editorial & 92 & $\begin{array}{l}\text { Dermal exposure: A decade of real } \\
\text { progress, Fenske RA }\end{array}$ & 2000 & & & $\checkmark$ & & \\
\hline \multirow[t]{4}{*}{$\begin{array}{l}\text { Technical publica- } \\
\text { tion/report }\end{array}$} & 114 & $\begin{array}{l}\text { European Chemical Industry Council } \\
\text { (CEFIC) Workshop on methods to } \\
\text { determine dermal permeation for } \\
\text { human risk assessment, Institute of } \\
\text { Medicine (IOM) }\end{array}$ & 2004 & & & & $\checkmark$ & \\
\hline & 129 & $\begin{array}{l}\text { Epidemiology of skin and respiratory } \\
\text { diseases among hairdressers, FIOH }\end{array}$ & 2001 & $\checkmark$ & $\checkmark$ & & & \\
\hline & 152 & $\begin{array}{l}\text { NIOSH pocket guide to chemical haz- } \\
\text { ards, NIOSH }\end{array}$ & 2004 & & & & & $\checkmark$ \\
\hline & 226 & $\begin{array}{l}\text { IPCS: Environmental health criteria doc- } \\
\text { ument on dermal absorption, WHO }\end{array}$ & 2005 & & & & $\checkmark$ & \\
\hline \multirow[t]{2}{*}{ Web page } & 19 & $\begin{array}{l}\text { Toxicological profile information sheet, } \\
\text { ATSDR }\end{array}$ & 2005 & & $\checkmark$ & $\checkmark$ & & \\
\hline & 155 & $\begin{array}{l}\text { Hazardous Substances Data Bank } \\
\text { (HSDB) [Web site], NLM }\end{array}$ & 2005 & & & & & $\checkmark$ \\
\hline
\end{tabular}

*See footnotes at end of table.

(Continued) 
PROFESSIONAL AUDIENCE

Table 4A (Continued). Overview of the investigation and control of occupational skin exposures

\begin{tabular}{|c|c|c|c|c|c|c|c|c|}
\hline \multirow[b]{2}{*}{ Resource type } & \multirow[b]{2}{*}{ ID } & \multirow[b]{2}{*}{ Title, author } & \multirow[b]{2}{*}{ Yr } & \multicolumn{5}{|c|}{ Subtopics } \\
\hline & & & & A. $1^{*}$ & A. 2 & A.3 & A.4 & A.5 \\
\hline $\begin{array}{l}\text { Web page } \\
\text { (Continued) }\end{array}$ & 220 & $\begin{array}{l}\text { Dermatitis: Safety and health assess- } \\
\text { ment and research for prevention } \\
\text { (SHARP) [Home page], WADLI }\end{array}$ & 2005 & $\checkmark$ & & $\checkmark$ & $\checkmark$ & \\
\hline \multirow[t]{11}{*}{ Web site } & 16 & CPI [Web site], ACC & 2006 & & $\checkmark$ & & & \\
\hline & 18 & ATSDR [Home page], ATSDR & 2005 & $\checkmark$ & & & & \\
\hline & 22 & AAFP [Home page], AAFP & 2005 & $\checkmark$ & $\checkmark$ & & & \\
\hline & 25 & AIHA [Home page], AIHA & 2006 & & & & & $\checkmark$ \\
\hline & 82 & $\begin{array}{l}\text { Dermatological engineering [Web site], } \\
\text { Enviroderm Services }\end{array}$ & 2005 & $\checkmark$ & $\checkmark$ & & $\checkmark$ & \\
\hline & 105 & Skin at work [Web site], HSE & 2005 & $\checkmark$ & $\checkmark$ & $\checkmark$ & $\checkmark$ & \\
\hline & 112 & ILO [Home page], ILO & 2005 & $\checkmark$ & $\checkmark$ & $\checkmark$ & $\checkmark$ & $\checkmark$ \\
\hline & 153 & NIOSH [Home page], NIOSH & 2005 & $\checkmark$ & $\checkmark$ & $\checkmark$ & & $\checkmark$ \\
\hline & 154 & $\begin{array}{l}\text { TOXNET-Databases on toxicology, } \\
\text { hazardous chemicals, environmen- } \\
\text { tal health, and toxic releases [Home } \\
\text { page], NLM }\end{array}$ & 2005 & $\checkmark$ & $\checkmark$ & $\checkmark$ & & $\checkmark$ \\
\hline & 162 & OSHA [Home page], OSHA & 2005 & $\checkmark$ & $\checkmark$ & & & $\checkmark$ \\
\hline & 206 & $\begin{array}{l}\text { The Extension Toxicology Network } \\
\text { (EXTOXNET) [Web site], Ohio State } \\
\text { University }\end{array}$ & 2006 & & $\checkmark$ & & & $\checkmark$ \\
\hline
\end{tabular}

${ }^{\star}$ A.1 $=$ Occurrence of Skin Exposures in Workplace; A.2=Health Hazards Resulting from Skin Exposure to Chemicals; A.3=Investigation, Intervention, and Control of Occupational Skin Exposures; A.4=Skin Physiology and Function as a Barrier to Chemical Insults; A.5=Dermal Regulations and Skin Notations 


\section{Topic 4B. Surveillance and Clinical Aspects}

Public health surveillance is the ongoing systematic collection, analysis, and interpretation of health data for purposes of improving health and safety. $O c$ cupational surveillance studies involve the tracking of illnesses, injuries, exposures and hazards in the workplace. The resources provided in the following table review information on dermal exposure surveillance work as well as provide clinical guidance on collecting data for surveillance studies, including information on assessing health effects from skin exposure to chemicals in the workplace.

\section{Subtopic B.1. Surveillance Studies Reporting Incidences of Occupa- tional Skin Exposures}

These resources summarize or refer to surveillance studies that report the incidence or prevalence of occupational skin exposures to chemicals. These may be studies where skin exposure to chemicals was a major focus of the study, or a minor focus of the surveillance study.

\section{Subtopic B.2. Loss of Workdays and Impact on Productivity}

These resources review information on lost workdays due to skin exposure health effects or review information on the impact of skin exposure health effects on worker productivity. This may include information on workers' compensation claims associated with skin exposure health effects.

\section{Subtopic B.3. Surveillance Study Protocols/Procedures for Gather- ing Data}

These resources contain guidance for collecting representative data for surveillance studies, including standard protocols or prevailing procedures used in surveillance studies.

\section{Subtopic B.4. Clinical Protocols for Recognition of Skin Exposure Health Effects}

These resources contain guidance for standard protocols or prevailing procedures used in clinical examinations that facilitate the recognition and identification of harmful health effects that result from skin exposures to chemicals. 


\section{PROFESSIONAL AUDIENCE \\ Table 4B. Surveillance and clinical aspects}

\begin{tabular}{|c|c|c|c|c|c|c|c|}
\hline \multirow[b]{2}{*}{ Resource type } & \multirow[b]{2}{*}{ ID } & \multirow[b]{2}{*}{ Title, author } & \multirow[b]{2}{*}{ Yr } & \multicolumn{4}{|c|}{ Subtopics } \\
\hline & & & & B. $1^{\star}$ & B. 2 & B.3 & B.4 \\
\hline \multirow[t]{3}{*}{$\begin{array}{l}\text { Book/monograph, } \\
\text { chapter }\end{array}$} & 28 & $\begin{array}{l}\text { Systemic toxicity from percutaneous absorp- } \\
\text { tion, Andersen KE }\end{array}$ & 1999 & $\checkmark$ & & & \\
\hline & 40 & $\begin{array}{l}\text { Chemical protective clothing and the skin: } \\
\text { Practical considerations, Boeniger M }\end{array}$ & 2002 & $\checkmark$ & & & \\
\hline & 221 & $\begin{array}{l}\text { Development of occupational skin disease, } \\
\text { Weber LW }\end{array}$ & 2003 & & & & $\checkmark$ \\
\hline $\begin{array}{l}\text { Book/monograph, } \\
\text { whole }\end{array}$ & 137 & $\begin{array}{l}\text { Contact and occupational dermatology, } \\
\text { Marks JG }\end{array}$ & 2002 & & & & $\checkmark$ \\
\hline \multirow[t]{2}{*}{ Brochure, pamphlet } & 60 & $\begin{array}{l}\text { A safety and health practitioner's guide to } \\
\text { skin protection, CPWR }\end{array}$ & 2000 & $\checkmark$ & $\checkmark$ & $\checkmark$ & $\checkmark$ \\
\hline & 61 & $\begin{array}{l}\text { Physician's alert for occupational contact der- } \\
\text { matitis among construction workers, CPWR }\end{array}$ & 2001 & & & & $\checkmark$ \\
\hline \multirow[t]{4}{*}{$\begin{array}{l}\text { Journal article- } \\
\text { primary }\end{array}$} & 55 & $\begin{array}{l}\text { Occupational dermatitis causing days away } \\
\text { from work in U.S. private industry, 1993, } \\
\text { Burnett CA }\end{array}$ & 1998 & $\checkmark$ & $\checkmark$ & $\checkmark$ & \\
\hline & 68 & $\begin{array}{l}\text { Surveillance of occupational skin disease: } \\
\text { EPIDERM and OPRA, Cherry N }\end{array}$ & 2000 & $\checkmark$ & & & \\
\hline & 131 & $\begin{array}{l}\text { Skin exposure to aliphatic polyisocyanates in } \\
\text { the auto body repair and refinishing indus- } \\
\text { try: A qualitative assessment, Liu Y }\end{array}$ & 2007 & $\checkmark$ & & & \\
\hline & 196 & $\begin{array}{l}\text { Occupational contact dermatitis to nickel: } \\
\text { experience of the British dermatologists } \\
\text { (EPIDERM) and occupational physicians } \\
\text { (OPRA) surveillance schemes, Shum KW }\end{array}$ & 2003 & $\checkmark$ & & & \\
\hline $\begin{array}{l}\text { Journal article- } \\
\text { review, } \\
\text { meta-analysis }\end{array}$ & 1 & $\begin{array}{l}\text { Skin lesions and environmental exposures. } \\
\text { An overview for the occupational health } \\
\text { nurse, ATSDR }\end{array}$ & 1996 & & & & $\checkmark$ \\
\hline
\end{tabular}

${ }^{\star}$ See footnotes at end of table.

(Continued) 


\section{PROFESSIONAL AUDIENCE Table 4B (Continued). Surveillance and clinical aspects}

\begin{tabular}{|c|c|c|c|c|c|c|c|}
\hline \multirow[b]{2}{*}{ Resource type } & \multirow[b]{2}{*}{ ID } & \multirow[b]{2}{*}{ Title, author } & \multirow[b]{2}{*}{ Yr } & \multicolumn{4}{|c|}{ Subtopics } \\
\hline & & & & B. $1^{\star}$ & B. 2 & B.3 & B.4 \\
\hline \multirow{13}{*}{$\begin{array}{l}\text { Journal article- } \\
\text { review, } \\
\text { meta-analysis } \\
\text { (Continued) }\end{array}$} & 27 & $\begin{array}{l}\text { Occupational issues of allergic contact der- } \\
\text { matitis, Andersen KE }\end{array}$ & 2003 & $\checkmark$ & & & $\checkmark$ \\
\hline & 30 & Occupational contact dermatitis, Antezana M & 2003 & & & & $\checkmark$ \\
\hline & 38 & $\begin{array}{l}\text { Skin exposure to isocyanates: Reasons for } \\
\text { concern, Bello D }\end{array}$ & 2007 & $\checkmark$ & & & \\
\hline & 42 & $\begin{array}{l}\text { In-use testing and interpretation of chemical- } \\
\text { resistant glove performance, Boeniger MF }\end{array}$ & 2002 & & $\checkmark$ & & \\
\hline & 53 & $\begin{array}{l}\text { Strategies for prevention: Occupational } \\
\text { contact dermatitis, Brown T }\end{array}$ & 2004 & $\checkmark$ & $\checkmark$ & $\checkmark$ & \\
\hline & 69 & $\begin{array}{l}\text { Occupational issues of irritant contact der- } \\
\text { matitis, Chew AL }\end{array}$ & 2003 & $\checkmark$ & $\checkmark$ & & $\checkmark$ \\
\hline & 73 & $\begin{array}{l}\text { Pesticide-related illness among migrant farm } \\
\text { workers in the United States, Das R }\end{array}$ & 2001 & $\checkmark$ & & & \\
\hline & 74 & $\begin{array}{l}\text { Beryllium exposure: Dermal and immuno- } \\
\text { logical considerations, Day GA }\end{array}$ & 2006 & $\checkmark$ & & & \\
\hline & 76 & $\begin{array}{l}\text { What can we learn from epidemiological } \\
\text { studies on irritant contact dermatitis? } \\
\text { Diepgen TL }\end{array}$ & 1995 & $\checkmark$ & & $\checkmark$ & \\
\hline & 77 & $\begin{array}{l}\text { Epidemiological studies on the prevention of } \\
\text { occupational contact dermatitis, Diepgen TL }\end{array}$ & 1996 & $\checkmark$ & & & \\
\hline & 78 & $\begin{array}{l}\text { The epidemiology of occupational contact } \\
\text { dermatitis, Diepgen TL }\end{array}$ & 1999 & $\checkmark$ & & & \\
\hline & 80 & $\begin{array}{l}\text { Occupational contact dermatitis I: Incidence } \\
\text { and return to work pressures, Emmett EA }\end{array}$ & 2002 & $\checkmark$ & $\checkmark$ & & \\
\hline & 81 & $\begin{array}{l}\text { Occupational contact dermatitis II: Risk as- } \\
\text { sessment and prognosis, Emmett EA }\end{array}$ & 2003 & $\checkmark$ & & & $\checkmark$ \\
\hline
\end{tabular}

*See footnotes at end of table.

(Continued) 


\section{PROFESSIONAL AUDIENCE \\ Table 4B (Continued). Surveillance and clinical aspects}

\begin{tabular}{|c|c|c|c|c|c|c|c|}
\hline \multirow[b]{2}{*}{ Resource type } & \multirow[b]{2}{*}{ ID } & \multirow[b]{2}{*}{ Title, author } & \multirow[b]{2}{*}{ Yr } & \multicolumn{4}{|c|}{ Subtopics } \\
\hline & & & & B. $1^{\star}$ & B.2 & B.3 & B.4 \\
\hline \multirow{12}{*}{$\begin{array}{l}\text { Journal article- } \\
\text { review, } \\
\text { meta-analysis } \\
\text { (Continued) }\end{array}$} & 101 & $\begin{array}{l}\text { Carbonless copy paper and workplace safety: } \\
\text { A review, Graves CG }\end{array}$ & 2000 & $\checkmark$ & & & \\
\hline & 103 & $\begin{array}{l}\text { Occupation-related allergies in dentistry, } \\
\text { Hamann CP }\end{array}$ & 2005 & $\checkmark$ & & & \\
\hline & 104 & $\begin{array}{l}\text { Textile dye allergic contact dermatitis preva- } \\
\text { lence, Hatch KL }\end{array}$ & 2000 & $\checkmark$ & & & \\
\hline & 122 & $\begin{array}{l}\text { A critique of assumptions about selecting } \\
\text { chemical-resistant gloves: A case for work- } \\
\text { place evaluation of glove efficacy, Klingner } \\
\text { TD }\end{array}$ & 2002 & $\checkmark$ & & & \\
\hline & 128 & $\begin{array}{l}\text { Toxicity of methyl methacrylate in dentistry, } \\
\text { Leggat PA }\end{array}$ & 2003 & $\checkmark$ & & & \\
\hline & 133 & $\begin{array}{l}\text { The epidemiology of occupational contact } \\
\text { dermatitis, Lushniak BD }\end{array}$ & 1995 & $\checkmark$ & $\checkmark$ & $\checkmark$ & \\
\hline & 134 & $\begin{array}{l}\text { The importance of occupational skin dis- } \\
\text { eases in the United States, Lushniak BD }\end{array}$ & 2003 & $\checkmark$ & $\checkmark$ & & \\
\hline & 135 & Occupational contact dermatitis, Lushniak BD & 2004 & $\checkmark$ & $\checkmark$ & & \\
\hline & 146 & $\begin{array}{l}\text { Differences between the sexes with regard to } \\
\text { work-related skin disease, Meding B }\end{array}$ & 2000 & $\checkmark$ & & & \\
\hline & 175 & $\begin{array}{l}\text { Clues to an accurate diagnosis of contact } \\
\text { dermatitis, Rietschel RL }\end{array}$ & 2004 & $\checkmark$ & & & \\
\hline & 199 & $\begin{array}{l}\text { Nordic Occupational Skin Questionnaire-2002 } \\
\text { (NOSQ-2002): A new tool for surveying } \\
\text { occupational skin diseases and exposure, } \\
\text { Susitaival P }\end{array}$ & 2003 & & & $\checkmark$ & \\
\hline & 203 & $\begin{array}{l}\text { Management of dermatitis in the rubber } \\
\text { manufacturing industry, Toeppen-Sprigg B }\end{array}$ & 1999 & $\checkmark$ & & & \\
\hline
\end{tabular}

*See footnotes at end of table.

(Continued) 


\section{PROFESSIONAL AUDIENCE \\ Table 4B (Continued). Surveillance and clinical aspects}

\begin{tabular}{|c|c|c|c|c|c|c|c|}
\hline \multirow[b]{2}{*}{ Resource type } & \multirow[b]{2}{*}{ ID } & \multirow[b]{2}{*}{ Title, author } & \multirow[b]{2}{*}{ Yr } & \multicolumn{4}{|c|}{ Subtopics } \\
\hline & & & & B. $1^{\star}$ & B.2 & B.3 & B.4 \\
\hline \multirow{2}{*}{$\begin{array}{l}\text { Journal article- } \\
\text { review, } \\
\text { meta-analysis } \\
\text { (Continued) }\end{array}$} & 223 & $\begin{array}{l}\text { Occupational contact dermatitis in the tex- } \\
\text { tile industry, Wigger-Alberti W }\end{array}$ & 2003 & $\checkmark$ & & & \\
\hline & 224 & The dermal toxicity of cement, Winder C & 2002 & $\checkmark$ & $\checkmark$ & & \\
\hline $\begin{array}{l}\text { Technical publica- } \\
\text { tion/report }\end{array}$ & 129 & $\begin{array}{l}\text { Epidemiology of skin and respiratory dis- } \\
\text { eases among hairdressers, FIOH }\end{array}$ & 2001 & $\checkmark$ & & $\checkmark$ & \\
\hline \multirow[t]{3}{*}{ Web page } & 54 & $\begin{array}{l}\text { Bureau of Labor Statistics (BLS) industry ill- } \\
\text { ness and injury data [Web site], BLS }\end{array}$ & 2005 & $\checkmark$ & $\checkmark$ & $\checkmark$ & \\
\hline & 155 & HSDB [Web site], NLM & 2005 & $\checkmark$ & & & \\
\hline & 220 & $\begin{array}{l}\text { Dermatitis: safety and health assessment and } \\
\text { research for prevention (SHARP) } \\
\text { [Home page] WADLI }\end{array}$ & 2005 & $\checkmark$ & $\checkmark$ & $\checkmark$ & \\
\hline \multirow[t]{8}{*}{ Web site } & 18 & ATSDR [Home page], ATSDR & 2005 & & & & $\checkmark$ \\
\hline & 57 & $\begin{array}{l}\text { California Division of Labor Statistics and } \\
\text { Research [Home page], California Depart- } \\
\text { ment of Industrial Relations [CA DIR] }\end{array}$ & 2003 & $\checkmark$ & $\checkmark$ & & \\
\hline & 59 & CPWR [Home page], CPWR & 2006 & $\checkmark$ & $\checkmark$ & & \\
\hline & 82 & $\begin{array}{l}\text { Dermatological engineering [Web site], } \\
\text { Enviroderm Services }\end{array}$ & 2005 & & & $\checkmark$ & $\checkmark$ \\
\hline & 87 & $\begin{array}{l}\text { United States Environmental Protection } \\
\text { Agency [Home page], USEPA }\end{array}$ & 2005 & $\checkmark$ & $\checkmark$ & $\checkmark$ & \\
\hline & 105 & Skin at work [Web site], HSE & 2005 & $\checkmark$ & $\checkmark$ & & $\checkmark$ \\
\hline & 112 & IW [Home page], ILO & 2005 & $\checkmark$ & $\checkmark$ & & \\
\hline & 153 & NIOSH [Home page], NIOSH & 2005 & $\checkmark$ & $\checkmark$ & & \\
\hline
\end{tabular}

*See footnotes at end of table.

(Continued) 


\section{PROFESSIONAL AUDIENCE \\ Table 4B (Continued). Surveillance and clinical aspects}

\begin{tabular}{|c|c|c|c|c|c|c|c|}
\hline \multirow[b]{2}{*}{ Resource type } & \multirow[b]{2}{*}{ ID } & \multirow[b]{2}{*}{ Title, author } & \multirow[b]{2}{*}{ Yr } & \multicolumn{4}{|c|}{ Subtopics } \\
\hline & & & & B. $1^{\star}$ & B.2 2 & B.3 & B.4 \\
\hline \multirow[t]{4}{*}{$\begin{array}{l}\text { Web site } \\
\text { (Continued) }\end{array}$} & 154 & $\begin{array}{l}\text { TOXNET-Databases on toxicology, hazard- } \\
\text { ous chemicals, environmental health, and } \\
\text { toxic releases [Home page], NLM }\end{array}$ & 2005 & & & & $\checkmark$ \\
\hline & 164 & $\begin{array}{l}\text { Oregon Worker Illness and Injury Preven- } \\
\text { tion Program (OWIIPP), Oregon Depart- } \\
\text { ment of Human Services (ORDHS) }\end{array}$ & 2005 & $\checkmark$ & $\checkmark$ & $\checkmark$ & \\
\hline & 165 & OR-OSHA [Home page], OR-OSHA & 2006 & $\checkmark$ & & & \\
\hline & 225 & IPCS [Home page], WHO & 2005 & & & & $\checkmark$ \\
\hline
\end{tabular}

${ }^{\star}$ B.1 $=$ Surveillance Studies Reporting Incidences of Occupational Skin Exposures; B.2=Loss of Workdays and Impact on Productivity; B.3=Surveillance Study Protocols/Procedures for Gathering Data; B.4=Clinical Protocols for Recognition of Skin Exposure Health Effects

\section{Topic 4C. Exposure Characterization}

Exposure characterization, a component of exposure assessment, is the process of describing the conditions of a given occupational environment that may influence exposure. These conditions may include the source of the chemical; the magnitude, frequency, duration, and routes of the exposure to the chemical; the chemical and physical properties of the chemical; and work practices, or how work using the chemical is performed in a given working environment. The resources found in this table provide information associated with dermal exposure characterization to chemicals.

\section{Subtopic C.1. Workplace Factors Associated with Harmful Skin Exposures}

These resources contain information on workplace factors that influence the potential for skin exposure to chemicals in the workplace, including the tasks performed, industrial processes in which workers are engaged, chemicals used or produced, and occupations or job titles of individuals involved in that work. 


\section{Subtopic C.2. Description of Factors Influencing Exposure Conditions}

These resources provide quantitative descriptions of environmental factors that influence the potential for skin exposure, including exposure duration and frequency; exposure to chemical mixtures; the concentration of the chemical(s) to which the worker is exposed; the affected skin surface area that is exposed; and factors associated with chemical uptake through the skin.

\section{Subtopic C.3. Checklists/Questionnaires to Quantify Skin Exposure Incidents}

These resources contain either questionnaires, checklists or other tools or descriptions of tools that can be used to aid in the collection of quantitative exposure data by professionals, as well as in the reporting and characterization of skin exposures using quantitative data.

\section{Subtopic C4. Direct Methods to Measure Exposures}

These resources contain descriptions of methods that can be used to measure exposure. Exposure can be evaluated by measuring chemical contamination of the workplace environment surfaces on which skin contact occurs; measuring exposure to the skin by sampling the skin or skin surrogates, such as body suits, patches, tapes and strips; or by visualization techniques and performing biomonitoring.

\section{Subtopic C.5. Exposure Modeling}

These resources review exposure characterization methods using modeling based on predictive algorithms developed experimentally or using exposure estimates developed from exposure modeling.

\section{PROFESSIONAL AUDIENCE}

Table 4C. Exposure characterization

\begin{tabular}{|c|c|c|c|c|c|c|c|c|}
\hline \multirow[b]{2}{*}{ Resource type } & \multirow[b]{2}{*}{ ID } & \multirow[b]{2}{*}{ Title, author } & \multirow[b]{2}{*}{ Yr } & \multicolumn{5}{|c|}{ Subtopics } \\
\hline & & & & C. $1^{\star}$ & C. 2 & C.3 & C.4 & C.5 \\
\hline $\begin{array}{l}\text { Book/monograph, } \\
\text { chapter }\end{array}$ & 4 & $\begin{array}{l}\text { Occupational skin exposure-absorption } \\
\text { of chemical agents and assessment of } \\
\text { exposures, Harris R }\end{array}$ & 2000 & & $\checkmark$ & & & \\
\hline & 5 & Dermal exposure modeling, Keil CB & 2000 & & $\checkmark$ & & & $\checkmark$ \\
\hline
\end{tabular}

\footnotetext{
${ }^{\star}$ See footnotes at end of table.
} 


\section{PROFESSIONAL AUDIENCE \\ Table 4C (Continued). Exposure characterization}

\begin{tabular}{|c|c|c|c|c|c|c|c|c|}
\hline \multirow[b]{2}{*}{ Resource type } & \multirow[b]{2}{*}{ ID } & \multirow[b]{2}{*}{ Title, author } & \multirow[b]{2}{*}{ Yr } & \multicolumn{5}{|c|}{ Subtopics } \\
\hline & & & & C. $1^{*}$ & C.2 & C.3 & C. 4 & C.5 \\
\hline \multirow{6}{*}{$\begin{array}{l}\text { Book/monograph, } \\
\text { chapter } \\
\text { (Continued) }\end{array}$} & 28 & $\begin{array}{l}\text { Systemic toxicity from percutaneous } \\
\text { absorption, Andersen KE }\end{array}$ & 1999 & $\checkmark$ & $\checkmark$ & & & \\
\hline & 90 & $\begin{array}{l}\text { Approaches for occupational dermal ex- } \\
\text { posure assessment and management, } \\
\text { Fehrenbacher MC }\end{array}$ & 2003 & & $\checkmark$ & & $\checkmark$ & $\checkmark$ \\
\hline & 158 & Surface and dermal monitoring, Ness SA & 2000 & & $\checkmark$ & $\checkmark$ & $\checkmark$ & $\checkmark$ \\
\hline & 171 & Health risk assessment, Paustenbach D & 1999 & & $\checkmark$ & & $\checkmark$ & $\checkmark$ \\
\hline & 185 & Dermal exposure assessments, Sahmel J & 2006 & & $\checkmark$ & & $\checkmark$ & $\checkmark$ \\
\hline & 221 & $\begin{array}{l}\text { Development of occupational skin dis- } \\
\text { ease, Weber LW }\end{array}$ & 2003 & & $\checkmark$ & & & \\
\hline \multirow{5}{*}{$\begin{array}{l}\text { Book/monograph, } \\
\text { whole }\end{array}$} & 17 & Occupational skin disease, Adams RM & 1999 & & $\checkmark$ & & $\checkmark$ & $\checkmark$ \\
\hline & 137 & $\begin{array}{l}\text { Contact and occupational dermatology, } \\
\text { Marks JG }\end{array}$ & 2002 & & & & $\checkmark$ & \\
\hline & 157 & $\begin{array}{l}\text { Surface and dermal monitoring for } \\
\text { toxic exposures, Ness SA }\end{array}$ & 1994 & & $\checkmark$ & $\checkmark$ & $\checkmark$ & \\
\hline & 218 & $\begin{array}{l}\text { Health risk assessment: Dermal and } \\
\text { inhalation exposure and absorption of } \\
\text { toxicants (dermatology), Wang RGM }\end{array}$ & 1993 & & $\checkmark$ & & & $\checkmark$ \\
\hline & 229 & Dermatotoxicology, Zhai H & 2004 & & $\checkmark$ & & & $\checkmark$ \\
\hline Brochure, pamphlet & 60 & $\begin{array}{l}\text { A safety and health practitioner's guide } \\
\text { to skin protection, CPWR }\end{array}$ & 2000 & $\checkmark$ & $\checkmark$ & $\checkmark$ & $\checkmark$ & \\
\hline Data file & 200 & $\begin{array}{l}\text { Syracuse Research Corporation (SRC)- } \\
\text { business areas: Environmental science } \\
\text { [Home page], SRC }\end{array}$ & 2006 & & $\checkmark$ & & $\checkmark$ & \\
\hline Guideline & 47 & $\begin{array}{l}\text { Guideline for hand hygiene in healthcare } \\
\text { Settings, CDC }\end{array}$ & 2002 & & & & & $\checkmark$ \\
\hline
\end{tabular}

${ }^{\star}$ See footnotes at end of table.

(Continued) 


\section{PROFESSIONAL AUDIENCE Table 4C (Continued). Exposure characterization}

\begin{tabular}{|c|c|c|c|c|c|c|c|c|}
\hline \multirow[b]{2}{*}{ Resource type } & \multirow[b]{2}{*}{ ID } & \multirow[b]{2}{*}{ Title, author } & \multirow[b]{2}{*}{ Yr } & \multicolumn{5}{|c|}{ Subtopics } \\
\hline & & & & C. $1^{*}$ & C. 2 & C.3 & C. 4 & C.5 \\
\hline \multirow[t]{2}{*}{$\begin{array}{l}\text { Guideline } \\
\text { (Continued) }\end{array}$} & 84 & $\begin{array}{l}\text { Organisation of Economic Co-opera- } \\
\text { tion and Development (OECD) series } \\
\text { on testing and assessment, number 28: } \\
\text { Guidance document for the conduct } \\
\text { of skin absorption studies, OECD }\end{array}$ & 2004 & & $\checkmark$ & & & \\
\hline & 161 & $\begin{array}{l}\text { Sampling for surface contamination, } \\
\text { OSHA }\end{array}$ & 2005 & & & & $\checkmark$ & \\
\hline \multirow[t]{7}{*}{$\begin{array}{l}\text { Journal article- } \\
\text { primary }\end{array}$} & 31 & $\begin{array}{l}\text { Effect of personal hygiene on blood lead } \\
\text { levels of workers at a lead processing } \\
\text { facility, Askin DP }\end{array}$ & 1997 & $\checkmark$ & & & $\checkmark$ & \\
\hline & 37 & $\begin{array}{l}\text { Slow curing of aliphatic polyisocyanate } \\
\text { paints in automotive refinishing: A po- } \\
\text { tential source for skin exposure, Bello D }\end{array}$ & 2007 & & $\checkmark$ & & $\checkmark$ & \\
\hline & 50 & $\begin{array}{l}\text { A dermal model for spray painters. Part } \\
\text { I: Subjective exposure modeling of } \\
\text { spray paint deposition, Brouwer DH }\end{array}$ & 2001 & $\checkmark$ & $\checkmark$ & & & $\checkmark$ \\
\hline & 65 & $\begin{array}{l}\text { Total body burden arising from a week's } \\
\text { repeated dermal exposure to N,N- } \\
\text { dimethylformamide (DMF) }\end{array}$ & 2005 & & $\checkmark$ & & $\checkmark$ & \\
\hline & 94 & $\begin{array}{l}\text { Modeling dermal exposure-an illustra- } \\
\text { tion for spray painting applications, } \\
\text { Flynn MR }\end{array}$ & 2006 & & & & & $\checkmark$ \\
\hline & 96 & $\begin{array}{l}\text { An overview of human exposure } \\
\text { modeling activities at the USEPA Na- } \\
\text { tional Exposure Research Laboratory } \\
\text { (NERL), Furtaw EJ Jr }\end{array}$ & 2001 & & & & & $\checkmark$ \\
\hline & 131 & $\begin{array}{l}\text { Skin exposure to aliphatic polyisocyanates } \\
\text { in the auto body repair and refinishing } \\
\text { industry: A qualitative assessment, Liu Y }\end{array}$ & 2007 & & $\checkmark$ & & $\checkmark$ & \\
\hline
\end{tabular}

${ }^{\star}$ See footnotes at end of table. 


\section{PROFESSIONAL AUDIENCE Table 4C (Continued). Exposure characterization}

\begin{tabular}{|c|c|c|c|c|c|c|c|c|}
\hline \multirow[b]{2}{*}{ Resource type } & \multirow[b]{2}{*}{ ID } & \multirow[b]{2}{*}{ Title, author } & \multirow[b]{2}{*}{ Yr } & \multicolumn{5}{|c|}{ Subtopics } \\
\hline & & & & C. $1^{*}$ & C. 2 & C.3 & C. 4 & C. 5 \\
\hline \multirow[t]{6}{*}{$\begin{array}{l}\text { Journal article- } \\
\text { primary } \\
\text { (Continued) }\end{array}$} & 142 & $\begin{array}{l}\text { Dermal exposure and urinary } \\
\text { 1-hydroxypyrene among asphalt roof- } \\
\text { ing workers, McClean MD }\end{array}$ & 2007 & $\checkmark$ & $\checkmark$ & & $\checkmark$ & \\
\hline & 195 & $\begin{array}{l}\text { A dermal model for spray painters. } \\
\text { Part II: Estimating the deposition and } \\
\text { uptake of solvents, Semple S }\end{array}$ & 2001 & $\checkmark$ & $\checkmark$ & & & \\
\hline & 202 & $\begin{array}{l}\text { A real-time in-vivo method for study- } \\
\text { ing the percutaneous absorption of } \\
\text { volatile chemicals, Thrall KD }\end{array}$ & 2000 & & $\checkmark$ & & $\checkmark$ & $\checkmark$ \\
\hline & 214 & $\begin{array}{l}\text { DREAM: A method for semi-quantitative } \\
\text { dermal exposure assessment, } \\
\text { van-Wendel-de-Joode B }\end{array}$ & 2003 & & & & & $\checkmark$ \\
\hline & 215 & $\begin{array}{l}\text { Reliability of a semi-quantitative meth- } \\
\text { od for dermal exposure assessment } \\
\text { (DREAM), van-Wendel-de-Joode B }\end{array}$ & 2005 & & & & $\checkmark$ & $\checkmark$ \\
\hline & 227 & $\begin{array}{l}\text { Rapid method for determining dermal } \\
\text { exposures to pesticides by use of tape } \\
\text { stripping and FTIR spectroscopy: A } \\
\text { pilot study, Wu CF }\end{array}$ & 2007 & & & & $\checkmark$ & \\
\hline \multirow[t]{4}{*}{$\begin{array}{l}\text { Journal article- } \\
\text { review, meta- } \\
\text { analysis }\end{array}$} & 1 & $\begin{array}{l}\text { Skin lesions and environmental expo- } \\
\text { sures. An overview for the occupa- } \\
\text { tional health nurse, ATSDR }\end{array}$ & 1996 & & & & $\checkmark$ & \\
\hline & 27 & $\begin{array}{l}\text { Occupational issues of allergic contact } \\
\text { dermatitis, Andersen KE }\end{array}$ & 2003 & & & & $\checkmark$ & \\
\hline & 30 & $\begin{array}{l}\text { Occupational contact dermatitis, Ante- } \\
\text { zana M }\end{array}$ & 2003 & & & & $\checkmark$ & \\
\hline & 38 & $\begin{array}{l}\text { Skin exposure to isocyanates: reasons } \\
\text { for concern, Bello D }\end{array}$ & 2007 & $\checkmark$ & $\checkmark$ & & $\checkmark$ & \\
\hline
\end{tabular}

${ }^{\star}$ See footnotes at end of table. 
PROFESSIONAL AUDIENCE Table 4C (Continued). Exposure characterization

\begin{tabular}{|c|c|c|c|c|c|c|c|c|}
\hline \multirow[b]{2}{*}{ Resource type } & \multirow[b]{2}{*}{ ID } & \multirow[b]{2}{*}{ Title, author } & \multirow[b]{2}{*}{ Yr } & \multicolumn{5}{|c|}{ Subtopics } \\
\hline & & & & C. $1^{*}$ & C.2 & C.3 & C. 4 & C.5 \\
\hline \multirow{10}{*}{$\begin{array}{l}\text { Journal article- } \\
\text { review, meta- } \\
\text { analysis } \\
\text { (Continued) }\end{array}$} & 39 & $\begin{array}{l}\text { Dermal route in systemic exposure, } \\
\text { Benford DJ }\end{array}$ & 1999 & & $\checkmark$ & & $\checkmark$ & $\checkmark$ \\
\hline & 42 & $\begin{array}{l}\text { In-use testing and interpretation of } \\
\text { chemical-resistant glove performance, } \\
\text { Boeniger MF }\end{array}$ & 2002 & & & & $\checkmark$ & $\checkmark$ \\
\hline & 44 & $\begin{array}{l}\text { Percutaneous absorption of organic } \\
\text { solvents, Boman A }\end{array}$ & 2000 & & $\checkmark$ & & & $\checkmark$ \\
\hline & 46 & $\begin{array}{l}\text { Proposal for the assessment of quan- } \\
\text { titative dermal exposure limits in } \\
\text { occupational environments: Part } 1 . \\
\text { Development of a concept to derive } \\
\text { a quantitative dermal occupational } \\
\text { exposure limit, Bos PM }\end{array}$ & 1998 & & $\checkmark$ & & & \\
\hline & 48 & $\begin{array}{l}\text { To what extent are biomonitoring data } \\
\text { available in chemical risk assessment? } \\
\text { Brondeau MT }\end{array}$ & 1999 & & $\sqrt{ }$ & & $\checkmark$ & \\
\hline & 49 & $\begin{array}{l}\text { Hand wash and manual skin wipes, } \\
\text { Brouwer DH }\end{array}$ & 2000 & & & & $\checkmark$ & \\
\hline & 51 & $\begin{array}{l}\text { Concepts of skin protection: Consider- } \\
\text { ations for the evaluation and termi- } \\
\text { nology of the performance of skin } \\
\text { protective equipment, Brouwer DH }\end{array}$ & 2005 & & $\checkmark$ & & & $\checkmark$ \\
\hline & 56 & $\begin{array}{l}\text { Suction methods for assessing contami- } \\
\text { nation on surfaces, Byrne MA }\end{array}$ & 2000 & & & & $\checkmark$ & $\checkmark$ \\
\hline & 67 & $\begin{array}{l}\text { Use of qualitative and quantitative fluo- } \\
\text { rescence techniques to assess dermal } \\
\text { exposure, Cherrie JW }\end{array}$ & 2000 & & & & $\checkmark$ & \\
\hline & 69 & $\begin{array}{l}\text { Occupational issues of irritant contact } \\
\text { dermatitis, Chew AL }\end{array}$ & 2003 & & & & $\checkmark$ & \\
\hline
\end{tabular}

*See footnotes at end of table.

(Continued) 
PROFESSIONAL AUDIENCE Table 4C (Continued). Exposure characterization

\begin{tabular}{|c|c|c|c|c|c|c|c|c|}
\hline \multirow[b]{2}{*}{ Resource type } & \multirow[b]{2}{*}{ ID } & \multirow[b]{2}{*}{ Title, author } & \multirow[b]{2}{*}{ Yr } & \multicolumn{5}{|c|}{ Subtopics } \\
\hline & & & & C. $1^{*}$ & C. 2 & C.3 & C. 4 & C. 5 \\
\hline \multirow{11}{*}{$\begin{array}{l}\text { Journal article- } \\
\text { review, meta- } \\
\text { analysis } \\
\text { (Continued) }\end{array}$} & 76 & $\begin{array}{l}\text { What can we learn from epidemiologi- } \\
\text { cal studies on irritant contact dermati- } \\
\text { tis? Diepgen TL }\end{array}$ & 1995 & $\checkmark$ & & & & \\
\hline & 81 & $\begin{array}{l}\text { Occupational contact dermatitis II: risk } \\
\text { assessment and prognosis, Emmett EA }\end{array}$ & 2003 & & $\checkmark$ & & $\checkmark$ & \\
\hline & 91 & $\begin{array}{l}\text { Dermal exposure assessment tech- } \\
\text { niques, Fenske RA }\end{array}$ & 1993 & & $\checkmark$ & & $\checkmark$ & $\checkmark$ \\
\hline & 93 & $\begin{array}{l}\text { Modelling skin permeability in risk } \\
\text { assessment-the future, Fitzpatrick D }\end{array}$ & 2004 & & $\checkmark$ & & & $\checkmark$ \\
\hline & 97 & $\begin{array}{l}\text { Developing COSHH Essentials: dermal } \\
\text { exposure, personal protective equip- } \\
\text { ment and first aid, Garrod AN }\end{array}$ & 2003 & & $\checkmark$ & & & $\checkmark$ \\
\hline & 104 & $\begin{array}{l}\text { Textile dye allergic contact dermatitis } \\
\text { prevalence, Hatch KL }\end{array}$ & 2000 & & & & $\checkmark$ & \\
\hline & 107 & $\begin{array}{l}\text { Factors determining percutaneous } \\
\text { metal absorption, Hostynek JJ }\end{array}$ & 2003 & & $\checkmark$ & & & \\
\hline & 115 & $\begin{array}{l}\text { Dermal absorption of benzene: implica- } \\
\text { tions for work practices and regula- } \\
\text { tions, Kalnas J }\end{array}$ & 2000 & & $\checkmark$ & & & $\checkmark$ \\
\hline & 120 & $\begin{array}{l}\text { Improved estimation of dermal pesti- } \\
\text { cide dose to agricultural workers upon } \\
\text { reentry, Kissel J }\end{array}$ & 2000 & & $\checkmark$ & & & $\checkmark$ \\
\hline & 121 & $\begin{array}{l}\text { Factors affecting soil adherence to skin } \\
\text { in hand-press trials, Kissel JC }\end{array}$ & 1996 & & $\checkmark$ & & & \\
\hline & 124 & $\begin{array}{l}\text { Occupational contact dermatitis. Rec- } \\
\text { ognition and management, Koch P }\end{array}$ & 2001 & & $\checkmark$ & & & \\
\hline
\end{tabular}

*See footnotes at end of table.

(Continued) 
PROFESSIONAL AUDIENCE Table 4C (Continued). Exposure characterization

\begin{tabular}{|c|c|c|c|c|c|c|c|c|}
\hline \multirow[b]{2}{*}{ Resource type } & \multirow[b]{2}{*}{ ID } & \multirow[b]{2}{*}{ Title, author } & \multirow[b]{2}{*}{ Yr } & \multicolumn{5}{|c|}{ Subtopics } \\
\hline & & & & C. $1^{*}$ & C. 2 & C. 3 & C. 4 & C. 5 \\
\hline \multirow{11}{*}{$\begin{array}{l}\text { Journal article- } \\
\text { review, meta- } \\
\text { analysis } \\
\text { (Continued) }\end{array}$} & 126 & $\begin{array}{l}\text { Temporal, personal and spatial variabil- } \\
\text { ity in dermal exposure, Kromhout H }\end{array}$ & 2001 & $\checkmark$ & $\checkmark$ & & & $\checkmark$ \\
\hline & 130 & $\begin{array}{l}\text { Techniques for estimating the percu- } \\
\text { taneous absorption of chemicals due } \\
\text { to occupational and environmental } \\
\text { exposure, Leung H-W }\end{array}$ & 1994 & & $\checkmark$ & & $\checkmark$ & $\checkmark$ \\
\hline & 132 & $\begin{array}{l}\text { Percutaneous absorption of arsenic from } \\
\text { environmental media, Lowney YW }\end{array}$ & 2005 & & $\checkmark$ & & & \\
\hline & 135 & $\begin{array}{l}\text { Occupational contact dermatitis, Lush- } \\
\text { niak BD }\end{array}$ & 2004 & $\checkmark$ & & & & \\
\hline & 138 & $\begin{array}{l}\text { Harmonization of future needs for der- } \\
\text { mal exposure assessment and model- } \\
\text { ing: A workshop report, Marquart H }\end{array}$ & 2001 & & $\checkmark$ & & $\checkmark$ & $\checkmark$ \\
\hline & 139 & $\begin{array}{l}\text { Determinants of dermal exposure } \\
\text { relevant for exposure modeling in } \\
\text { regulatory risk assessment, Marquart J }\end{array}$ & 2003 & $\checkmark$ & $\checkmark$ & & & $\checkmark$ \\
\hline & 141 & $\begin{array}{l}\text { Dermal Measurement and Wipe Sam- } \\
\text { pling Methods: A Review, McArthur B }\end{array}$ & 1992 & & & & $\checkmark$ & \\
\hline & 143 & $\begin{array}{l}\text { Assessment of dermal absorption and } \\
\text { penetration of components of a fuel } \\
\text { mixture (JP-8), McDougal JN }\end{array}$ & 2002 & & $\checkmark$ & & & \\
\hline & 144 & $\begin{array}{l}\text { Methods for assessing risks of der- } \\
\text { mal exposures in the workplace, } \\
\text { McDougal JN }\end{array}$ & 2002 & & $\checkmark$ & & & $\checkmark$ \\
\hline & 148 & $\begin{array}{l}\text { Quantitative structure-permeability } \\
\text { relationships (QSPRs) for percutane- } \\
\text { ous absorption, Moss GP }\end{array}$ & 2002 & & $\checkmark$ & & & \\
\hline & 160 & $\begin{array}{l}\text { New approaches for assessment of oc- } \\
\text { cupational exposure to metals using } \\
\text { on-site measurements, Nygren O }\end{array}$ & 2002 & & & & $\checkmark$ & \\
\hline
\end{tabular}

*See footnotes at end of table. 
PROFESSIONAL AUDIENCE Table 4C (Continued). Exposure characterization

\begin{tabular}{|c|c|c|c|c|c|c|c|c|}
\hline \multirow[b]{2}{*}{ Resource type } & \multirow[b]{2}{*}{ ID } & \multirow[b]{2}{*}{ Title, author } & \multirow[b]{2}{*}{ Yr } & \multicolumn{5}{|c|}{ Subtopics } \\
\hline & & & & C. $1^{*}$ & C.2 & C.3 & C.4 & C.5 \\
\hline \multirow{13}{*}{$\begin{array}{l}\text { Journal article- } \\
\text { review, meta- } \\
\text { analysis } \\
\text { (Continued) }\end{array}$} & 163 & $\begin{array}{l}\text { A toolkit for dermal risk assessment and } \\
\text { management: an overview, Oppl R }\end{array}$ & 2003 & & & & & $\checkmark$ \\
\hline & 172 & $\begin{array}{l}\text { Assessment of dermal exposure-em- } \\
\text { pirical models and indicative distribu- } \\
\text { tions, Phillips AM }\end{array}$ & 2001 & & & & & $\checkmark$ \\
\hline & 173 & Assessing dermal absorption, Poet TS & 2000 & & $\checkmark$ & & & \\
\hline & 175 & $\begin{array}{l}\text { Clues to an accurate diagnosis of con- } \\
\text { tact dermatitis, Rietschel RL }\end{array}$ & 2004 & & & & $\checkmark$ & \\
\hline & 177 & $\begin{array}{l}\text { Quantitating absorption of complex } \\
\text { chemical mixtures, Riviere JE }\end{array}$ & 2004 & & $\checkmark$ & & & \\
\hline & 182 & $\begin{array}{l}\text { Conservatism in pesticide exposure as- } \\
\text { sessment, Ross JH }\end{array}$ & 2000 & & $\checkmark$ & & & \\
\hline & 183 & Solvents and the skin, Rowse DH & 2004 & & $\checkmark$ & & & \\
\hline & 184 & $\begin{array}{l}\text { A distributed parameter physiological- } \\
\text { ly-based pharmacokinetic model for } \\
\text { dermal and inhalation exposure to } \\
\text { volatile organic compounds, Roy A }\end{array}$ & 1996 & & $\checkmark$ & & & $\checkmark$ \\
\hline & 187 & $\begin{array}{l}\text { Percutaneous penetration studies for } \\
\text { risk assessment, Sartorelli P }\end{array}$ & 2000 & & $\checkmark$ & & & \\
\hline & 188 & $\begin{array}{l}\text { Dermal exposure assessment in occupa- } \\
\text { tional medicine, Sartorelli P }\end{array}$ & 2002 & & $\checkmark$ & & & \\
\hline & 190 & $\begin{array}{l}\text { Conceptual model for assessment of } \\
\text { dermal exposure, Schneider T }\end{array}$ & 1999 & & $\checkmark$ & & $\checkmark$ & $\checkmark$ \\
\hline & 191 & Dermal exposure assessment, Schneider T & 2000 & & & & $\checkmark$ & $\checkmark$ \\
\hline & 194 & $\begin{array}{l}\text { Dermal exposure to chemicals in the } \\
\text { workplace: Just how important is skin } \\
\text { absorption? Semple S }\end{array}$ & 2004 & & $\checkmark$ & & $\checkmark$ & $\checkmark$ \\
\hline
\end{tabular}

*See footnotes at end of table.

(Continued) 
PROFESSIONAL AUDIENCE Table 4C (Continued). Exposure characterization

\begin{tabular}{|c|c|c|c|c|c|c|c|c|}
\hline \multirow[b]{2}{*}{ Resource type } & \multirow[b]{2}{*}{ ID } & \multirow[b]{2}{*}{ Title, author } & \multirow[b]{2}{*}{ Yr } & \multicolumn{5}{|c|}{ Subtopics } \\
\hline & & & & C. $1^{*}$ & C. 2 & C. 3 & C. 4 & C. 5 \\
\hline \multirow{10}{*}{$\begin{array}{l}\text { Journal article- } \\
\text { review, meta- } \\
\text { analysis } \\
\text { (Continued) }\end{array}$} & 197 & $\begin{array}{l}\text { From xenobiotic chemistry and metabo- } \\
\text { lism to better prediction and risk assess- } \\
\text { ment of skin allergy, Smith Pease CK }\end{array}$ & 2003 & & $\checkmark$ & & & \\
\hline & 198 & $\begin{array}{l}\text { Use of patches and whole body sam- } \\
\text { pling for the assessment of dermal } \\
\text { exposure, Soutar A }\end{array}$ & 2000 & & & & $\checkmark$ & $\checkmark$ \\
\hline & 204 & $\begin{array}{l}\text { Prediction of irritancy in the human } \\
\text { skin irritancy model and occupational } \\
\text { setting, Tupker RA }\end{array}$ & 2003 & & $\checkmark$ & & $\checkmark$ & \\
\hline & 210 & $\begin{array}{l}\text { From dermal exposure to internal dose, } \\
\text { van de Sandt J }\end{array}$ & 2008 & & & & $\checkmark$ & $\checkmark$ \\
\hline & 211 & $\begin{array}{l}\text { Assessment of dermal exposure to } \\
\text { chemicals, van Hemmen JJ }\end{array}$ & 1995 & & $\checkmark$ & & $\checkmark$ & \\
\hline & 213 & $\begin{array}{l}\text { Review of skin permeation hazard of } \\
\text { bitumen fumes, van Rooij JG }\end{array}$ & 2008 & & $\checkmark$ & & & \\
\hline & 216 & $\begin{array}{l}\text { Dermal exposure assessment in oc- } \\
\text { cupational epidemiologic research, } \\
\text { Vermeulen R }\end{array}$ & 2002 & & $\checkmark$ & & & $\checkmark$ \\
\hline & 219 & $\begin{array}{l}\text { Deriving default dermal exposure val- } \\
\text { ues for use in a risk assessment toolkit } \\
\text { for small and medium-sized enter- } \\
\text { prises, Warren N }\end{array}$ & 2003 & & & & & $\checkmark$ \\
\hline & 222 & $\begin{array}{l}\text { Understanding percutaneous absorp- } \\
\text { tion for occupational health and } \\
\text { safety, Wester RC }\end{array}$ & 2000 & & $\checkmark$ & & & $\checkmark$ \\
\hline & 228 & $\begin{array}{l}\text { Toxic effects of chemical mixtures, } \\
\text { Zeliger HI }\end{array}$ & 2003 & & $\checkmark$ & & & \\
\hline
\end{tabular}

${ }^{\star}$ See footnotes at end of table.

(Continued) 


\section{PROFESSIONAL AUDIENCE Table 4C (Continued). Exposure characterization}

\begin{tabular}{|c|c|c|c|c|c|c|c|c|}
\hline \multirow[b]{2}{*}{ Resource type } & \multirow[b]{2}{*}{ ID } & \multirow[b]{2}{*}{ Title, author } & \multirow[b]{2}{*}{ Yr } & \multicolumn{5}{|c|}{ Subtopics } \\
\hline & & & & C. $1^{*}$ & C.2 & C. 3 & C.4 & C. 5 \\
\hline $\begin{array}{l}\text { Other- } \\
\text { commentary }\end{array}$ & 41 & $\begin{array}{l}\text { Exposure and absorption of hazardous } \\
\text { materials through the skin, Boeniger MF }\end{array}$ & 2000 & & $\checkmark$ & & & \\
\hline \multirow[t]{9}{*}{$\begin{array}{l}\text { Technical publica- } \\
\text { tion/report }\end{array}$} & 10 & $\begin{array}{l}\text { Risk Assessment Guidance for Super- } \\
\text { fund (RAGS), Volume I: Human } \\
\text { Health Evaluation Manual (Part E, } \\
\text { Supplemental Guidance for Dermal } \\
\text { Risk Assessment) }\end{array}$ & 2001 & & $\checkmark$ & & & $\checkmark$ \\
\hline & 36 & $\begin{array}{l}\text { Dermal absorption of cutting fluid mix- } \\
\text { tures, Baynes RE }\end{array}$ & 2003 & & $\checkmark$ & & & \\
\hline & 66 & $\begin{array}{l}\text { Occupational dermal exposure assess- } \\
\text { ment: A review of methodologies and } \\
\text { field data-final report, Chen CK }\end{array}$ & 1996 & & & & $\checkmark$ & $\checkmark$ \\
\hline & 86 & $\begin{array}{l}\text { Summary report for the workshop on } \\
\text { issues associated with dermal exposure } \\
\text { and uptake, USEPA, Bethesda, MD, } \\
\text { December 10-11, 1998, United States }\end{array}$ & 2000 & & $\checkmark$ & & $\checkmark$ & $\checkmark$ \\
\hline & 89 & $\begin{array}{l}\text { Skin and respiratory sensitizers: refer- } \\
\text { ence chemicals data bank, European } \\
\text { Centre for Ecotoxicology and Toxicol- } \\
\text { ogy of Chemicals (ECETOC) }\end{array}$ & 1999 & & & & & $\checkmark$ \\
\hline & 102 & $\begin{array}{l}\text { Prediction and assessment of dermal } \\
\text { exposure, Guy R }\end{array}$ & 1998 & & $\checkmark$ & & & $\checkmark$ \\
\hline & 108 & $\begin{array}{l}\text { Dermal and non-dietary ingestion } \\
\text { exposure workshop: NERL Human } \\
\text { Exposure Research Program, Hubal EC }\end{array}$ & 1998 & & $\checkmark$ & & $\checkmark$ & $\checkmark$ \\
\hline & 114 & $\begin{array}{l}\text { CEFIC workshop on methods to deter- } \\
\text { mine dermal permeation for human } \\
\text { risk assessment, IOM }\end{array}$ & 2004 & & $\checkmark$ & & & $\checkmark$ \\
\hline & 129 & $\begin{array}{l}\text { Epidemiology of skin and respiratory } \\
\text { diseases among hairdressers, FIOH }\end{array}$ & 2001 & $\checkmark$ & & & $\checkmark$ & \\
\hline
\end{tabular}

${ }^{\star}$ See footnotes at end of table.

(Continued) 


\section{PROFESSIONAL AUDIENCE Table 4C (Continued). Exposure characterization}

\begin{tabular}{|c|c|c|c|c|c|c|c|c|}
\hline \multirow[b]{2}{*}{ Resource type } & \multirow[b]{2}{*}{ ID } & \multirow[b]{2}{*}{ Title, author } & \multirow[b]{2}{*}{ Yr } & \multicolumn{5}{|c|}{ Subtopics } \\
\hline & & & & C. $1^{\star}$ & C.2 & C.3 & C. 4 & C.5 \\
\hline \multirow[t]{4}{*}{$\begin{array}{l}\text { Technical publi- } \\
\text { cation/report } \\
\text { (Continued) }\end{array}$} & 176 & $\begin{array}{l}\text { Percutaneous absorption of chemical } \\
\text { mixtures relevant to the Gulf War, } \\
\text { Riviere JE }\end{array}$ & 2002 & & $\checkmark$ & & & \\
\hline & 178 & $\begin{array}{l}\text { Quantitating the percutaneous ab- } \\
\text { sorption of mechanistically defined } \\
\text { chemical mixtures final report } 15 \text { Nov } \\
\text { 1997-14 Nov 2000, Riviere JE }\end{array}$ & 2001 & & $\checkmark$ & & & \\
\hline & 179 & $\begin{array}{l}\text { Quantitating the percutaneous ab- } \\
\text { sorption of mechanistically defined } \\
\text { chemical mixtures final report } 15 \mathrm{Dec} \\
\text { 2000-14 Dec 2003, Riviere JE }\end{array}$ & 2004 & $\checkmark$ & $\checkmark$ & & & \\
\hline & 226 & $\begin{array}{l}\text { IPCS: Environmental health criteria } \\
\text { document on dermal absorption } \\
\text { [Draft], WHO }\end{array}$ & 2005 & & $\checkmark$ & & & \\
\hline \multirow[t]{6}{*}{ Web page } & 19 & $\begin{array}{l}\text { Toxicological profile information sheet } \\
\text { [Web site], ATSDR }\end{array}$ & 2005 & & & & $\checkmark$ & \\
\hline & 85 & $\begin{array}{l}\text { Harmonized test guidelines [Web site], } \\
\text { USEPA }\end{array}$ & 1998 & & & & $\checkmark$ & \\
\hline & 155 & HSDB [Web site], NLM & 2005 & $\checkmark$ & & & & \\
\hline & 167 & $\begin{array}{l}\text { OECD's database on chemical risk as- } \\
\text { sessment models, OECD }\end{array}$ & 2006 & & & & & $\checkmark$ \\
\hline & 209 & $\begin{array}{l}\text { USEPA, Office of Pollution Prevention } \\
\text { and Toxics (OPPT): Exposure assess- } \\
\text { ment tools and models [Home page], } \\
\text { USEPA }\end{array}$ & 2005 & & & & & $\checkmark$ \\
\hline & 220 & $\begin{array}{l}\text { Dermatitis: Safety and health assess- } \\
\text { ment and research for prevention } \\
\text { (SHARP) [Home page] WADLI }\end{array}$ & 2005 & $\sqrt{ }$ & & & & \\
\hline Web site & 16 & CPI [Web site], ACC & 2006 & $\checkmark$ & & & & \\
\hline
\end{tabular}

${ }^{\star}$ See footnotes at end of table.

(Continued) 
PROFESSIONAL AUDIENCE

Table 4C (Continued). Exposure characterization

\begin{tabular}{|c|c|c|c|c|c|c|c|c|}
\hline \multirow[b]{2}{*}{ Resource type } & \multirow[b]{2}{*}{ ID } & \multirow[b]{2}{*}{ Title, author } & \multirow[b]{2}{*}{ Yr } & \multicolumn{5}{|c|}{ Subtopics } \\
\hline & & & & C. $1^{\star}$ & C.2 & C.3 & C. 4 & C.5 \\
\hline \multirow[t]{10}{*}{$\begin{array}{l}\text { Web site } \\
\quad \text { (Continued) }\end{array}$} & 32 & $\begin{array}{l}\text { American Society for Testing and Mate- } \\
\text { rials (ASTM) International } \\
\text { [Home page], ASTM International }\end{array}$ & 2006 & $\checkmark$ & & & $\checkmark$ & \\
\hline & 71 & $\begin{array}{l}\text { The pioneer in the reduction of dermal } \\
\text { exposure [Colormetric Laboratories, } \\
\text { Inc. Home page], Colormetric Labo- } \\
\text { ratories, Inc. }\end{array}$ & 2005 & & & & $\checkmark$ & \\
\hline & 82 & $\begin{array}{l}\text { Dermatological engineering [Web site], } \\
\text { Enviroderm Services }\end{array}$ & 2005 & $\checkmark$ & $\checkmark$ & & $\checkmark$ & \\
\hline & 87 & USEPA [Home page], USEPA & 2005 & & $\checkmark$ & & $\checkmark$ & $\checkmark$ \\
\hline & 105 & Skin at work [Web site], HSE & 2005 & $\checkmark$ & $\checkmark$ & & $\checkmark$ & \\
\hline & 112 & ILO [Home page], ILO & 2005 & $\checkmark$ & $\checkmark$ & & & \\
\hline & 153 & NIOSH [Home page], NIOSH & 2005 & $\checkmark$ & $\checkmark$ & & $\checkmark$ & \\
\hline & 154 & $\begin{array}{l}\text { TOXNET_-Databases on toxicology, } \\
\text { hazardous chemicals, environmen- } \\
\text { tal health, and toxic releases [Home } \\
\text { page], NLM }\end{array}$ & 2005 & $\checkmark$ & & & & \\
\hline & 162 & OSHA [Home page], OSHA & 2005 & $\checkmark$ & $\checkmark$ & & $\checkmark$ & \\
\hline & 201 & $\begin{array}{l}\text { Wil ten Berge model for dermal absorp- } \\
\text { tion [Home page], ten Berge W }\end{array}$ & 2004 & & & & & $\checkmark$ \\
\hline
\end{tabular}

\section{Topic 4D. Hazard Identification from Toxicological Studies or Modeling}

Hazard identification, another component of exposure risk assessment, is the process of establishing the existence of a hazard through field observations and laboratory analysis of the exposures and adverse health effects. As part of this process, 
the nature of the hazard is determined, such as whether the chemicals causes skin irritation, skin corrosion, sensitization or some systemic toxic effect, and the doseresponse relationship under the conditions of exposure is determined. Resources found in the following tables review information associated with hazard identification based on toxicological studies or modeling efforts.

\section{Subtopic D.1. Potential Health Effects Resulting from Specific Chemicals}

These resources review or summarize information on potential health effects resulting from skin exposure to chemicals. Health effects can include localized skin irritation and corrosion, including irritant contact dermatitis; sensitization of the skin, including allergic contact dermatitis, or sensitization of the respiratory tract as a result of skin exposure; the potential contribution of skin exposure and resulting dermal absorption to systemic toxicity; or the contribution of skin exposure to overall or total exposure to a chemical(s).

\section{Subtopic D.1.a. Irritant Contact Dermatitis}

These resources address chemically induced irritant contact dermatitis. This form of dermatitis is caused by direct exposure of the skin to a chemical or other irritating substance. Irritation in the form of inflammation usually occurs either immediately or within a short period of time.

\section{Subtopic D.1.b. Allergic Contact Dermatitis/Sensitization}

Resources found here include information on allergic contact dermatitis, which is an immunologically mediated reaction of the skin caused by direct contact of the skin of a sensitized individual to a chemical that is an allergen. Inflammation also occurs, but only in sensitized individuals.

\section{Subtopic D.1.c. Systemic Toxicity}

Resources listed under this subtopic include information on dermal exposures that can cause systemic toxicity. Unlike irritant and allergic contact dermatitis, which cause localized toxic effects, systemic toxicity refers to chemicals absorbed through the skin which are then transported to other sites in the body where their toxic effects occur. It is possible for a chemical to produce both a local and systemic adverse health effect.

\section{Subtopic D.1.d. Other Health Effects}

These resources contain information on other health effects that can be caused by dermal exposure and include conditions such as urticaria, which is immediately 
hypersensitivity; foreign body dermatitis, caused by foreign compounds such as fiber glass, silica, and asbestos penetrating the skin; pigmentation changes of the skin; cancer; and other health effects.

\section{Subtopic D.2. Summaries of Health Effects and Dose-Response Relationships}

These resources contain sources of data or actual databases that provide summaries and discuss the significance of health effects that result from skin exposures to chemicals in the workplace.

\section{Subtopic D.3. Characterization Protocols}

These resources contain protocols or guidelines for use in chemical hazard characterizations. Protocols and guidelines cited include those for various kinds of toxicological studies. The responses characterized in these protocols and guidelines include corrosivity, irritation potential, sensitization potential, and potential to cause systemic effects. Also included are resources that provide protocols and guidelines for measuring skin permeation rates and reservoir effects, as well as protocols for developing and validating qualitative and quantitative structure activity relationships QSAR for application in hazard identification and for use in validating QSAR as a screening tool to identify skin hazards in high priority chemicals.

\section{PROFESSIONAL AUDIENCE}

Table 4D(I). Hazard identification from toxicological studies or modeling

\begin{tabular}{|c|c|c|c|c|c|c|c|}
\hline \multirow[b]{2}{*}{ Resource type } & \multirow[b]{2}{*}{ ID } & \multirow[b]{2}{*}{ Title, author } & \multirow[b]{2}{*}{ Yr } & \multicolumn{4}{|c|}{ D Subtopics } \\
\hline & & & & D. $1 a^{*}$ & D.1b & D.1c & D.1d \\
\hline \multirow[t]{4}{*}{$\begin{array}{l}\text { Book/monograph, } \\
\text { chapter }\end{array}$} & 4 & $\begin{array}{l}\text { Occupational skin exposure-absorption } \\
\text { of chemical agents and assessment of } \\
\text { exposures, Harris R }\end{array}$ & 2000 & $\checkmark$ & $\checkmark$ & & \\
\hline & 28 & $\begin{array}{l}\text { Systemic toxicity from percutaneous ab- } \\
\text { sorption, Andersen KE }\end{array}$ & 1999 & & $\checkmark$ & $\checkmark$ & \\
\hline & 40 & $\begin{array}{l}\mathrm{CPC} \text { and the skin: Practical consider- } \\
\text { ations, Boeniger M }\end{array}$ & 2002 & $\checkmark$ & & & \\
\hline & 221 & $\begin{array}{l}\text { Development of occupational skin disease, } \\
\text { Weber LW }\end{array}$ & 2003 & $\checkmark$ & $\checkmark$ & & \\
\hline
\end{tabular}

\footnotetext{
${ }^{\star}$ See footnotes at end of table.
} 
PROFESSIONAL AUDIENCE Table 4D(I) (Continued). Hazard identification from toxicological studies or modeling

\begin{tabular}{|c|c|c|c|c|c|c|c|}
\hline \multirow[b]{2}{*}{ Resource type } & \multirow[b]{2}{*}{ ID } & \multirow[b]{2}{*}{ Title, author } & \multirow[b]{2}{*}{ Yr } & \multicolumn{4}{|c|}{ D Subtopics } \\
\hline & & & & D. $\mathrm{a}^{*}$ & D.1b & D.1c & D.1d \\
\hline \multirow{5}{*}{$\begin{array}{l}\text { Book/monograph, } \\
\text { whole }\end{array}$} & 17 & Occupational skin disease, Adams RM & 1999 & $\checkmark$ & $\checkmark$ & $\checkmark$ & $\checkmark$ \\
\hline & 117 & $\begin{array}{l}\text { Handbook of occupational dermatology, } \\
\text { Kanerva L }\end{array}$ & 2000 & $\checkmark$ & $\checkmark$ & $\sqrt{ }$ & \\
\hline & 137 & $\begin{array}{l}\text { Contact and occupational dermatology, } \\
\text { Marks JG }\end{array}$ & 2002 & $\checkmark$ & $\checkmark$ & & \\
\hline & 218 & $\begin{array}{l}\text { Health risk assessment: Dermal and } \\
\text { inhalation exposure and absorption of } \\
\text { toxicants (dermatology), Wang RGM }\end{array}$ & 1993 & & & & $\checkmark$ \\
\hline & 229 & Dermatotoxicology, Zhai H & 2004 & $\checkmark$ & $\checkmark$ & $\checkmark$ & $\checkmark$ \\
\hline $\begin{array}{l}\text { Brochure, } \\
\text { pamphlet }\end{array}$ & 60 & $\begin{array}{l}\text { A safety and health practitioner's guide to } \\
\text { skin protection, CPWR }\end{array}$ & 2000 & $\checkmark$ & $\checkmark$ & & $\checkmark$ \\
\hline Data file & 200 & $\begin{array}{l}\text { SRC_-business areas: Environmental sci- } \\
\text { ence [Home page], SRC }\end{array}$ & 2006 & $\checkmark$ & $\checkmark$ & & \\
\hline Guideline & 23 & $\begin{array}{l}\text { Documentation of the threshold limit val- } \\
\text { ues for chemical substances, ACGIH }\end{array}$ & 2001 & $\checkmark$ & $\checkmark$ & $\checkmark$ & $\checkmark$ \\
\hline \multirow[t]{4}{*}{$\begin{array}{l}\text { Journal article- } \\
\text { review, meta- } \\
\text { analysis }\end{array}$} & 1 & $\begin{array}{l}\text { Skin lesions and environmental exposures. } \\
\text { An overview for the occupational health } \\
\text { nurse, ATSDR }\end{array}$ & 1996 & $\checkmark$ & $\checkmark$ & $\checkmark$ & $\checkmark$ \\
\hline & 26 & $\begin{array}{l}\text { Cleansing without compromise: The } \\
\text { impact of cleansers on the skin barrier } \\
\text { and the technology of mild cleansing, } \\
\text { Ananthapadmanabhan KP }\end{array}$ & 2004 & $\checkmark$ & & & \\
\hline & 30 & $\begin{array}{l}\text { Occupational contact dermatitis, Antezana } \\
\text { M }\end{array}$ & 2003 & $\checkmark$ & $\checkmark$ & & \\
\hline & 34 & $\begin{array}{l}\text { Classification criteria for skin-sensitizing } \\
\text { chemicals: a commentary, Basketter DA }\end{array}$ & 1999 & $\checkmark$ & $\checkmark$ & $\checkmark$ & \\
\hline
\end{tabular}

${ }^{\star}$ See footnotes at end of table. 
PROFESSIONAL AUDIENCE Table 4D(I) (Continued). Hazard identification from toxicological studies or modeling

\begin{tabular}{|c|c|c|c|c|c|c|c|}
\hline \multirow[b]{2}{*}{ Resource type } & \multirow[b]{2}{*}{ ID } & \multirow[b]{2}{*}{ Title, author } & \multirow[b]{2}{*}{ Yr } & \multicolumn{4}{|c|}{ D Subtopics } \\
\hline & & & & $D .1 a^{*}$ & D.1b & D.1c & D.1d \\
\hline \multirow{11}{*}{$\begin{array}{l}\text { Journal article- } \\
\text { review, meta- } \\
\text { analysis } \\
\text { (Continued) }\end{array}$} & 38 & $\begin{array}{l}\text { Skin exposure to isocyanates: Reasons for } \\
\text { concern, Bello D }\end{array}$ & 2007 & & $\checkmark$ & & $\checkmark$ \\
\hline & 46 & $\begin{array}{l}\text { Proposal for the assessment of quantitative } \\
\text { dermal exposure limits in occupational } \\
\text { environments: Part 1. Development of a } \\
\text { concept to derive a quantitative dermal } \\
\text { occupational exposure limit, Bos PM }\end{array}$ & 1998 & $\checkmark$ & $\checkmark$ & & \\
\hline & 69 & $\begin{array}{l}\text { Occupational issues of irritant contact } \\
\text { dermatitis, Chew AL }\end{array}$ & 2003 & $\checkmark$ & & & \\
\hline & 74 & $\begin{array}{l}\text { Beryllium exposure: Dermal and immu- } \\
\text { nological considerations, Day GA }\end{array}$ & 2006 & & $\checkmark$ & $\checkmark$ & \\
\hline & 77 & $\begin{array}{l}\text { Epidemiological studies on the preven- } \\
\text { tion of occupational contact dermatitis, } \\
\text { Diepgen TL }\end{array}$ & 1996 & & $\checkmark$ & & \\
\hline & 78 & $\begin{array}{l}\text { The epidemiology of occupational contact } \\
\text { dermatitis, Diepgen TL }\end{array}$ & 1999 & $\checkmark$ & $\checkmark$ & & \\
\hline & 79 & $\begin{array}{l}\text { Skin-conditioning products in occupa- } \\
\text { tional dermatology, Elsner P }\end{array}$ & 2003 & $\checkmark$ & & & \\
\hline & 81 & $\begin{array}{l}\text { Occupational contact dermatitis II: Risk } \\
\text { assessment and prognosis, Emmett EA }\end{array}$ & 2003 & $\checkmark$ & $\checkmark$ & & \\
\hline & 99 & $\begin{array}{l}\text { A chemical dataset for evaluation of alter- } \\
\text { native approaches to skin-sensitization } \\
\text { testing, Gerberick GF }\end{array}$ & 2004 & & $\checkmark$ & & \\
\hline & 101 & $\begin{array}{l}\text { CCP and workplace safety: A review, } \\
\text { Graves CG }\end{array}$ & 2000 & $\checkmark$ & & & \\
\hline & 103 & $\begin{array}{l}\text { Occupation-related allergies in dentistry, } \\
\text { Hamann CP }\end{array}$ & 2005 & $\checkmark$ & $\checkmark$ & & \\
\hline
\end{tabular}

*See footnotes at end of table. 
PROFESSIONAL AUDIENCE Table 4D(I) (Continued). Hazard identification from toxicological studies or modeling

\begin{tabular}{|c|c|c|c|c|c|c|c|}
\hline \multirow[b]{2}{*}{ Resource type } & \multirow[b]{2}{*}{ ID } & \multirow[b]{2}{*}{ Title, author } & \multirow[b]{2}{*}{ Yr } & \multicolumn{4}{|c|}{ D Subtopics } \\
\hline & & & & $D^{1} 1 a^{*}$ & D.1b & D.1c & D.1d \\
\hline \multirow[t]{10}{*}{$\begin{array}{l}\text { Journal article- } \\
\text { review, meta- } \\
\text { analysis } \\
\text { (Continued) }\end{array}$} & 116 & $\begin{array}{l}\text { Dermatological aspects of a success- } \\
\text { ful introduction and continuation of } \\
\text { alcohol-based hand rubs for hygienic } \\
\text { hand disinfection, Kampf G }\end{array}$ & 2003 & $\checkmark$ & & & \\
\hline & 118 & $\begin{array}{l}\text { The role of the skin in the development } \\
\text { of chemical respiratory hypersensitivity, } \\
\text { Kimber I }\end{array}$ & 1996 & & $\checkmark$ & $\checkmark$ & $\checkmark$ \\
\hline & 119 & $\begin{array}{l}\text { Alternative approaches to the identifica- } \\
\text { tion and characterization of chemical } \\
\text { allergens, Kimber I }\end{array}$ & 2001 & & $\checkmark$ & & \\
\hline & 122 & $\begin{array}{l}\text { A critique of assumptions about select- } \\
\text { ing chemical-resistant gloves: A case for } \\
\text { workplace evaluation of glove efficacy, } \\
\text { Klingner TD }\end{array}$ & 2002 & $\checkmark$ & $\checkmark$ & $\checkmark$ & \\
\hline & 124 & $\begin{array}{l}\text { Occupational contact dermatitis. Recogni- } \\
\text { tion and management, Koch P }\end{array}$ & 2001 & $\checkmark$ & & & \\
\hline & 125 & $\begin{array}{l}\text { Occupational skin-protection products-a } \\
\text { review, Kresken J }\end{array}$ & 2003 & $\checkmark$ & $\checkmark$ & & \\
\hline & 127 & $\begin{array}{l}\text { Effectiveness of skin protection creams as a } \\
\text { preventive measure in occupational derma- } \\
\text { titis: A critical update according to criteria } \\
\text { of evidence-based medicine, Kutting B }\end{array}$ & 2003 & $\checkmark$ & & & \\
\hline & 128 & $\begin{array}{l}\text { Toxicity of methyl methacrylate in den- } \\
\text { tistry, Leggat PA }\end{array}$ & 2003 & & & $\checkmark$ & \\
\hline & 133 & $\begin{array}{l}\text { The epidemiology of occupational contact } \\
\text { dermatitis, Lushniak BD }\end{array}$ & 1995 & $\checkmark$ & & & \\
\hline & 135 & $\begin{array}{l}\text { Occupational contact dermatitis, Lushniak } \\
\text { BD }\end{array}$ & 2004 & $\checkmark$ & $\checkmark$ & & $\checkmark$ \\
\hline
\end{tabular}

${ }^{\star}$ See footnotes at end of table. 
PROFESSIONAL AUDIENCE Table 4D(I) (Continued). Hazard identification from toxicological studies or modeling

\begin{tabular}{|c|c|c|c|c|c|c|c|}
\hline \multirow[b]{2}{*}{ Resource type } & \multirow[b]{2}{*}{ ID } & \multirow[b]{2}{*}{ Title, author } & \multirow[b]{2}{*}{ Yr } & \multicolumn{4}{|c|}{ D Subtopics } \\
\hline & & & & D.1 $a^{*}$ & D.1b & D.1c & D.1d \\
\hline \multirow{11}{*}{$\begin{array}{l}\text { Journal article- } \\
\text { review, meta- } \\
\text { analysis } \\
\text { (Continued) }\end{array}$} & 140 & $\begin{array}{l}\text { Dermal toxicity due to industrial chemi- } \\
\text { cals, Mathur AK }\end{array}$ & 2002 & $\checkmark$ & $\checkmark$ & $\checkmark$ & $\checkmark$ \\
\hline & 146 & $\begin{array}{l}\text { Differences between the sexes with regard } \\
\text { to work-related skin disease, Meding B }\end{array}$ & 2000 & $\checkmark$ & $\checkmark$ & & \\
\hline & 175 & $\begin{array}{l}\text { Clues to an accurate diagnosis of contact } \\
\text { dermatitis, Rietschel RL }\end{array}$ & 2004 & $\checkmark$ & $\checkmark$ & & \\
\hline & 180 & $\begin{array}{l}\text { Quantitative structure-activity relation- } \\
\text { ships for predicting skin and respiratory } \\
\text { sensitization, Rodford R }\end{array}$ & 2003 & & $\checkmark$ & & \\
\hline & 183 & Solvents and the skin, Rowse DH & 2004 & $\checkmark$ & $\checkmark$ & $\checkmark$ & $\checkmark$ \\
\hline & 189 & $\begin{array}{l}\text { Chemical substances and contact } \\
\text { allergy- } 244 \text { substances ranked accord- } \\
\text { ing to allergenic potency, Schlede E }\end{array}$ & 2003 & & $\checkmark$ & & \\
\hline & 192 & $\begin{array}{l}\text { When should a substance be designated as } \\
\text { sensitizing for the skin ('Sh') or for the } \\
\text { airways ('Sa')?, Schnuch A }\end{array}$ & 2002 & & $\checkmark$ & & \\
\hline & 197 & $\begin{array}{l}\text { From xenobiotic chemistry and metabo- } \\
\text { lism to better prediction and risk assess- } \\
\text { ment of skin allergy, Smith Pease CK }\end{array}$ & 2003 & & $\checkmark$ & & \\
\hline & 213 & $\begin{array}{l}\text { Review of skin permeation hazard of bitu- } \\
\text { men fumes, van Rooij JG }\end{array}$ & 2008 & & & & $\checkmark$ \\
\hline & 223 & $\begin{array}{l}\text { Occupational contact dermatitis in the } \\
\text { textile industry, Wigger-Alberti W }\end{array}$ & 2003 & $\checkmark$ & $\checkmark$ & & \\
\hline & 224 & The dermal toxicity of cement, Winder C & 2002 & $\checkmark$ & $\checkmark$ & & $\checkmark$ \\
\hline $\begin{array}{l}\text { Technical publica- } \\
\text { tion/report }\end{array}$ & 36 & $\begin{array}{l}\text { Dermal absorption of cutting fluid mix- } \\
\text { tures, Baynes RE }\end{array}$ & 2003 & $\checkmark$ & & & \\
\hline
\end{tabular}

*See footnotes at end of table. 
PROFESSIONAL AUDIENCE Table 4D(I) (Continued). Hazard identification from toxicological studies or modeling

\begin{tabular}{|c|c|c|c|c|c|c|c|}
\hline \multirow[b]{2}{*}{ Resource type } & \multirow[b]{2}{*}{ ID } & \multirow[b]{2}{*}{ Title, author } & \multirow[b]{2}{*}{ Yr } & \multicolumn{4}{|c|}{ D Subtopics } \\
\hline & & & & D. $1 a^{*}$ & D.1b & D.1c & D.1d \\
\hline \multirow[t]{3}{*}{$\begin{array}{l}\text { Technical publica- } \\
\text { tion/report } \\
\text { (Continued) }\end{array}$} & 89 & $\begin{array}{l}\text { Skin and respiratory sensitizers: Reference } \\
\text { chemicals data bank, European Centre for } \\
\text { Ecotoxicology and Toxicology of Chemicals }\end{array}$ & 1999 & $\checkmark$ & $\checkmark$ & & \\
\hline & 129 & $\begin{array}{l}\text { Epidemiology of skin and respiratory dis- } \\
\text { eases among hairdressers, FIOH }\end{array}$ & 2001 & $\checkmark$ & $\checkmark$ & & $\checkmark$ \\
\hline & 179 & $\begin{array}{l}\text { Quantitating the percutaneous absorption of } \\
\text { mechanistically defined chemical mixtures, } \\
15 \text { Dec 2000-14 Dec 2003, Riviere JE }\end{array}$ & 2004 & & & $\checkmark$ & \\
\hline \multirow[t]{2}{*}{ Web page } & 19 & $\begin{array}{l}\text { Toxicological profile information sheet } \\
\text { [Web page], ATSDR }\end{array}$ & 2005 & $\checkmark$ & $\checkmark$ & $\checkmark$ & $\checkmark$ \\
\hline & 155 & HSDB [Web page], NLM & 2005 & $\checkmark$ & $\checkmark$ & $\sqrt{ }$ & $\sqrt{ }$ \\
\hline \multirow[t]{9}{*}{ Web site } & 16 & CPI [Web site], ACC & 2006 & & $\checkmark$ & & \\
\hline & 18 & ATSDR [Home page], ATSDR & 2005 & $\checkmark$ & $\checkmark$ & $\checkmark$ & $\checkmark$ \\
\hline & 22 & AAFP [Home page], AAFP & 2005 & $\checkmark$ & $\checkmark$ & & \\
\hline & 32 & $\begin{array}{l}\text { ASTM International [Home page], ASTM } \\
\text { International }\end{array}$ & 2006 & & $\checkmark$ & & \\
\hline & 82 & $\begin{array}{l}\text { Dermatological engineering [Web page], } \\
\text { Enviroderm Services }\end{array}$ & 2005 & $\checkmark$ & $\checkmark$ & $\checkmark$ & \\
\hline & 105 & Skin at work [Web page], HSE & 2005 & $\checkmark$ & $\checkmark$ & & \\
\hline & 110 & $\begin{array}{l}\text { Interagency Coordinating Committee on } \\
\text { the Validation of Alternative Methods } \\
\text { (ICCVAM) [Home page], ICCVAM }\end{array}$ & 2005 & & $\checkmark$ & & \\
\hline & 112 & ILO [Home page], ILO & 2005 & $\checkmark$ & $\checkmark$ & $\checkmark$ & \\
\hline & 153 & NIOSH [Home page], NIOSH & 2005 & $\checkmark$ & $\checkmark$ & $\checkmark$ & \\
\hline
\end{tabular}

${ }^{\star}$ See footnotes at end of table.

(Continued) 
PROFESSIONAL AUDIENCE Table 4D(I) (Continued). Hazard identification from toxicological studies or modeling

\begin{tabular}{|c|c|c|c|c|c|c|c|}
\hline \multirow[b]{2}{*}{ Resource type } & \multirow[b]{2}{*}{ ID } & \multirow[b]{2}{*}{ Title, author } & \multirow[b]{2}{*}{ Yr } & \multicolumn{4}{|c|}{ D Subtopics } \\
\hline & & & & D.1 $\mathrm{a}^{\star}$ & D.1b & D.1c & D.1d \\
\hline \multirow[t]{3}{*}{$\begin{array}{l}\text { Web site } \\
\text { (Continued) }\end{array}$} & 154 & $\begin{array}{l}\text { TOXNET_Databases on toxicology, haz- } \\
\text { ardous chemicals, environmental health, } \\
\text { and toxic releases [Home page], NLM }\end{array}$ & 2005 & $\checkmark$ & $\checkmark$ & $\checkmark$ & $\checkmark$ \\
\hline & 206 & $\begin{array}{l}\text { EXTOXNET [Web site], Ohio State Uni- } \\
\text { versity }\end{array}$ & 2006 & $\checkmark$ & $\checkmark$ & $\checkmark$ & \\
\hline & 225 & IPCS [Home page], WHO & 2005 & $\checkmark$ & $\checkmark$ & $\sqrt{ }$ & $\sqrt{ }$ \\
\hline
\end{tabular}

${ }^{*}$ D1=Potential Health Effects Resulting from Specific Chemicals; D1a=Irritant Contact Dermatitis; D1b=Allergic Contact Dermatitis/Sensitization; D1c=Systemic Toxicity; D1d=Other Health Effects

PROFESSIONAL AUDIENCE Table 4D(II). Hazard identification from toxicological studies or modeling

\begin{tabular}{|c|c|c|c|c|c|}
\hline \multirow[b]{2}{*}{ Resource type } & \multirow[b]{2}{*}{ ID } & \multirow[b]{2}{*}{ Title, author } & \multirow[b]{2}{*}{ Yr } & \multicolumn{2}{|c|}{ Subtopics } \\
\hline & & & & D. $2^{*}$ & D.3 \\
\hline $\begin{array}{l}\text { Book/monograph, } \\
\text { chapter }\end{array}$ & 171 & Health risk assessment, Paustenbach D & 1999 & $\checkmark$ & $\checkmark$ \\
\hline \multirow{3}{*}{$\begin{array}{l}\text { Book/monograph, } \\
\text { whole }\end{array}$} & 17 & Occupational skin disease, Adams RM & 1999 & & $\checkmark$ \\
\hline & 137 & Contact and occupational dermatology, Marks JG & 2002 & & $\checkmark$ \\
\hline & 218 & $\begin{array}{l}\text { Health risk assessment: dermal and inhalation ex- } \\
\text { posure and absorption of toxicants (dermatology), } \\
\text { Wang RGM }\end{array}$ & 1993 & $\checkmark$ & \\
\hline Data file & 200 & $\begin{array}{l}\text { SRC-business areas: environmental science [Home } \\
\text { page], SRC }\end{array}$ & 2006 & & $\checkmark$ \\
\hline Guideline & 2 & $\begin{array}{l}\text { Health effects test guidelines: OPPTS 870.2500, acute } \\
\text { dermal irritation, OPPTS }\end{array}$ & 1998 & & $\checkmark$ \\
\hline
\end{tabular}

${ }^{*}$ See footnotes at end of table.

(Continued) 


\section{PROFESSIONAL AUDIENCE Table 4D(II) (Continued). Hazard identification from toxicological studies or modeling}

\begin{tabular}{|c|c|c|c|c|c|}
\hline \multirow[b]{2}{*}{ Resource type } & \multirow[b]{2}{*}{ ID } & \multirow[b]{2}{*}{ Title, author } & \multirow[b]{2}{*}{ Yr } & \multicolumn{2}{|c|}{ Subtopics } \\
\hline & & & & D. $2^{*}$ & D.3 \\
\hline \multirow[t]{2}{*}{$\begin{array}{l}\text { Guideline } \\
\text { (Continued) }\end{array}$} & 23 & $\begin{array}{l}\text { Documentation of the threshold limit values for } \\
\text { chemical substances, ACGIH }\end{array}$ & 2001 & $\checkmark$ & \\
\hline & 84 & $\begin{array}{l}\text { OECD series on testing and assessment, number } 28 \text { : } \\
\text { guidance document for the conduct of skin absorp- } \\
\text { tion studies, OECD }\end{array}$ & 2004 & & $\checkmark$ \\
\hline \multirow[t]{4}{*}{$\begin{array}{l}\text { Journal article- } \\
\text { primary }\end{array}$} & 65 & $\begin{array}{l}\text { Total body burden arising from a week's repeated } \\
\text { dermal exposure to N,N-dimethylformamide } \\
\text { (DMF), Chang H }\end{array}$ & 2005 & $\checkmark$ & \\
\hline & 131 & $\begin{array}{l}\text { Skin exposure to aliphatic polyisocyanates in the } \\
\text { auto body repair and refinishing industry: A quali- } \\
\text { tative assessment, Liu Y }\end{array}$ & 2007 & & $\checkmark$ \\
\hline & 142 & $\begin{array}{l}\text { Dermal exposure and urinary 1-hydroxypyrene } \\
\text { among asphalt roofing workers, McClean MD }\end{array}$ & 2007 & & $\checkmark$ \\
\hline & 202 & $\begin{array}{l}\text { A real-time in-vivo method for studying the percuta- } \\
\text { neous absorption of volatile chemicals, Thrall KD }\end{array}$ & 2000 & $\checkmark$ & $\checkmark$ \\
\hline \multirow{6}{*}{$\begin{array}{l}\text { Journal article- } \\
\text { review, } \\
\text { meta-analysis }\end{array}$} & 27 & $\begin{array}{l}\text { Occupational issues of allergic contact dermatitis, } \\
\text { Andersen KE }\end{array}$ & 2003 & $\checkmark$ & \\
\hline & 35 & $\begin{array}{l}\text { Factors affecting thresholds in allergic contact } \\
\text { dermatitis: safety and regulatory considerations, } \\
\text { Basketter DA }\end{array}$ & 2002 & $\checkmark$ & \\
\hline & 39 & Dermal route in systemic exposure, Benford DJ & 1999 & & $\checkmark$ \\
\hline & 44 & Percutaneous absorption of organic solvents, Boman A & 2000 & $\checkmark$ & \\
\hline & 46 & $\begin{array}{l}\text { Proposal for the assessment of quantitative dermal } \\
\text { exposure limits in occupational environments: Part } \\
\text { 1. Development of a concept to derive a quantita- } \\
\text { tive dermal occupational exposure limit, Bos PM }\end{array}$ & 1998 & & $\checkmark$ \\
\hline & 77 & $\begin{array}{l}\text { Epidemiological studies on the prevention of occu- } \\
\text { pational contact dermatitis, Diepgen TL }\end{array}$ & 1996 & $\checkmark$ & \\
\hline
\end{tabular}

${ }^{\star}$ See footnotes at end of table.

(Continued) 


\section{PROFESSIONAL AUDIENCE Table 4D(II) (Continued). Hazard identification from toxicological studies or modeling}

\begin{tabular}{|c|c|c|c|c|c|}
\hline \multirow[b]{2}{*}{ Resource type } & \multirow[b]{2}{*}{ ID } & \multirow[b]{2}{*}{ Title, author } & \multirow[b]{2}{*}{ Yr } & \multicolumn{2}{|c|}{ Subtopics } \\
\hline & & & & D. $2^{*}$ & D.3 \\
\hline \multirow{13}{*}{$\begin{array}{l}\text { Journal article- } \\
\text { review, } \\
\text { meta-analysis } \\
\text { (Continued) }\end{array}$} & 78 & $\begin{array}{l}\text { The epidemiology of occupational contact dermati- } \\
\text { tis, Diepgen TL }\end{array}$ & 1999 & & $\checkmark$ \\
\hline & 81 & $\begin{array}{l}\text { Occupational contact dermatitis II: Risk assessment } \\
\text { and prognosis, Emmett EA }\end{array}$ & 2003 & $\checkmark$ & \\
\hline & 93 & $\begin{array}{l}\text { Modeling skin permeability in risk assessment-the } \\
\text { future, Fitzpatrick D }\end{array}$ & 2004 & & $\checkmark$ \\
\hline & 99 & $\begin{array}{l}\text { A chemical dataset for evaluation of alternative ap- } \\
\text { proaches to skin-sensitization testing, Gerberick GF }\end{array}$ & 2004 & & $\checkmark$ \\
\hline & 101 & CCP and workplace safety: A review, Graves CG & 2000 & & $\checkmark$ \\
\hline & 119 & $\begin{array}{l}\text { Alternative approaches to the identification and char- } \\
\text { acterization of chemical allergens, Kimber I }\end{array}$ & 2001 & & $\checkmark$ \\
\hline & 130 & $\begin{array}{l}\text { Techniques for estimating the percutaneous absorp- } \\
\text { tion of chemicals due to occupational and environ- } \\
\text { mental exposure, Leung H-W }\end{array}$ & 1994 & & $\checkmark$ \\
\hline & 148 & $\begin{array}{l}\text { Quantitative structure-permeability relationships } \\
\text { (QSPRs) for percutaneous absorption, Moss GP }\end{array}$ & 2002 & & $\checkmark$ \\
\hline & 170 & $\begin{array}{l}\text { Quantitative structure-activity relationships for pre- } \\
\text { dicting skin and eye irritation, Patlewicz G }\end{array}$ & 2003 & & $\checkmark$ \\
\hline & 175 & $\begin{array}{l}\text { Clues to an accurate diagnosis of contact dermatitis, } \\
\text { Rietschel RL }\end{array}$ & 2004 & $\checkmark$ & \\
\hline & 177 & $\begin{array}{l}\text { Quantitating absorption of complex chemical mix- } \\
\text { tures, Riviere JE }\end{array}$ & 2004 & & $\checkmark$ \\
\hline & 180 & $\begin{array}{l}\text { Quantitative structure-activity relationships for pre- } \\
\text { dicting skin and respiratory sensitization, Rodford R }\end{array}$ & 2003 & & $\checkmark$ \\
\hline & 183 & Solvents and the skin, Rowse DH & 2004 & $\checkmark$ & $\checkmark$ \\
\hline
\end{tabular}

${ }^{\star}$ See footnotes at end of table.

(Continued) 
PROFESSIONAL AUDIENCE Table 4D(II) (Continued). Hazard identification from toxicological studies or modeling

\begin{tabular}{|c|c|c|c|c|c|}
\hline \multirow[b]{2}{*}{ Resource type } & \multirow[b]{2}{*}{ ID } & \multirow[b]{2}{*}{ Title, author } & \multirow[b]{2}{*}{ Yr } & \multicolumn{2}{|c|}{ Subtopics } \\
\hline & & & & D. $2^{*}$ & D.3 \\
\hline \multirow{6}{*}{$\begin{array}{l}\text { Journal article- } \\
\text { review, } \\
\text { meta-analysis } \\
\text { (Continued) }\end{array}$} & 187 & $\begin{array}{l}\text { Percutaneous penetration studies for risk assessment, } \\
\text { Sartorelli P }\end{array}$ & 2000 & & $\checkmark$ \\
\hline & 189 & $\begin{array}{l}\text { Chemical substances and contact allergy-244 } \\
\text { substances ranked according to allergenic potency, } \\
\text { Schlede E }\end{array}$ & 2003 & & $\checkmark$ \\
\hline & 197 & $\begin{array}{l}\text { From xenobiotic chemistry and metabolism to bet- } \\
\text { ter prediction and risk assessment of skin allergy, } \\
\text { Smith Pease CK }\end{array}$ & 2003 & $\sqrt{ }$ & $\checkmark$ \\
\hline & 210 & $\begin{array}{l}\text { From dermal exposure to internal dose, van de Sandt } \\
\text { J, Dellarco M }\end{array}$ & 2008 & & $\checkmark$ \\
\hline & 217 & $\begin{array}{l}\text { Quantitative structure-activity relationships for pre- } \\
\text { dicting percutaneous absorption rates, Walker JD }\end{array}$ & 2003 & & $\checkmark$ \\
\hline & 228 & Toxic effects of chemical mixtures, Zeliger HI & 2003 & & $\checkmark$ \\
\hline \multirow{6}{*}{$\begin{array}{l}\text { Technical publication/ } \\
\text { report }\end{array}$} & 36 & Dermal absorption of cutting fluid mixtures, Baynes RE & 2003 & & $\checkmark$ \\
\hline & 66 & $\begin{array}{l}\text { Occupational dermal exposure assessment: a review of } \\
\text { methodologies and field data: Final report, Chen CK }\end{array}$ & 1996 & $\checkmark$ & \\
\hline & 114 & $\begin{array}{l}\text { CEFIC Workshop on methods to determine dermal } \\
\text { permeation for human risk assessment, IOM }\end{array}$ & 2004 & & $\checkmark$ \\
\hline & 176 & $\begin{array}{l}\text { Percutaneous absorption of chemical mixtures rel- } \\
\text { evant to the Gulf War, Riviere JE }\end{array}$ & 2002 & & $\checkmark$ \\
\hline & 178 & $\begin{array}{l}\text { Quantitating the percutaneous absorption of } \\
\text { mechanistically defined chemical mixtures: } 15 \text { Nov } \\
\text { 1997-14 Nov 2000, Riviere JE }\end{array}$ & 2001 & & $\checkmark$ \\
\hline & 179 & $\begin{array}{l}\text { Quantitating the percutaneous absorption of } \\
\text { mechanistically defined chemical mixtures: } 15 \text { Dec } \\
\text { 2000-14 Dec 2003, Riviere JE, Monteiro-Riviere NA }\end{array}$ & 2004 & $\checkmark$ & \\
\hline
\end{tabular}

*See footnotes at end of table. 
PROFESSIONAL AUDIENCE Table 4D(II) (Continued). Hazard identification from toxicological studies or modeling

\begin{tabular}{|c|c|c|c|c|c|}
\hline \multirow[b]{2}{*}{ Resource type } & \multirow[b]{2}{*}{ ID } & \multirow[b]{2}{*}{ Title, author } & \multirow[b]{2}{*}{ Yr } & \multicolumn{2}{|c|}{ Subtopics } \\
\hline & & & & D. $2^{\star}$ & D.3 \\
\hline $\begin{array}{l}\text { Technical publication/ } \\
\text { report } \\
\text { (Continued) }\end{array}$ & 226 & $\begin{array}{l}\text { The International Programme on Chemical Safety: } \\
\text { Environmental health criteria document on dermal } \\
\text { absorption [Draft], WHO }\end{array}$ & 2005 & & $\checkmark$ \\
\hline \multirow[t]{4}{*}{ Web page } & 19 & $\begin{array}{l}\text { Toxicological profile information sheet [Home page], } \\
\text { ATSDR }\end{array}$ & 2005 & $\checkmark$ & \\
\hline & 85 & Harmonized test guidelines [Web page], USEPA & 1998 & & $\checkmark$ \\
\hline & 166 & $\begin{array}{l}\text { Guidelines for the testing of chemicals [Web page], } \\
\text { OECD }\end{array}$ & 2005 & & $\checkmark$ \\
\hline & 167 & $\begin{array}{l}\text { OECD's database on chemical risk assessment mod- } \\
\text { els [Web page], OECD }\end{array}$ & 2006 & & $\checkmark$ \\
\hline \multirow[t]{8}{*}{ Web site } & 18 & ATSDR [Home page], ATSDR & 2005 & $\checkmark$ & \\
\hline & 87 & USEPA [Home page], USEPA & 2005 & & $\checkmark$ \\
\hline & 105 & Skin at work [Web site], HSE & 2005 & $\checkmark$ & $\checkmark$ \\
\hline & 110 & ICCVAM [Home page], ICCVAM & 2005 & & $\checkmark$ \\
\hline & 154 & $\begin{array}{l}\text { TOXNET-Databases on toxicology, hazardous } \\
\text { chemicals, environmental health, and toxic releases } \\
\text { [Home page], NLM }\end{array}$ & 2005 & $\checkmark$ & \\
\hline & 201 & $\begin{array}{l}\text { Wil ten Berge model for dermal absorption [Home } \\
\text { page], ten Berge W }\end{array}$ & 2004 & & $\checkmark$ \\
\hline & 206 & $\begin{array}{l}\text { EXTOXNET-The Extension Toxicology Network } \\
\text { [Web site], Ohio State University }\end{array}$ & 2006 & $\checkmark$ & \\
\hline & 225 & IPCS [Home page], WHO & 2005 & $\checkmark$ & $\checkmark$ \\
\hline
\end{tabular}

${ }^{*}$ D2=Summaries of Health Effects and Dose-Response Relationships; D3=Characterization Protocols 


\section{Topic 4E. Risk Assessment}

Risk assessment is the measurement or estimate of the chances of a given level of exposure to a chemical to cause harm. With respect to skin exposures, risk assessments are performed by workplace health and safety professionals to provide the employer with an estimate of the likelihood of an illness or injury to result from skin exposure to a chemical hazard. Risk assessment is essential for setting occupational safety and health priorities and for demonstrating health impairment when promulgating occupational standards. These resources contain information associated with skin exposure risk assessments.

\section{Subtopic E.1. Guidelines for Risk Assessment or Analysis}

The resources indicated in this column provide descriptions of risk assessments and guildlines for analyses to determine if skin exposure to a chemical likely to cause a given effect, either a localized health effect or a systemic health effect.

\section{Subtopic E.2. Example of Risk Assessments}

The resources identified in this column provide examples of risk assessments that have been conducted to evaluate hazards associated with a specific chemical or chemical mixture in addition to models used to evaluate risk of dermal exposures.

\section{PROFESSIONAL AUDIENCE}

Table 4E. Risk assessment

\begin{tabular}{|c|c|c|c|c|c|}
\hline \multirow[b]{2}{*}{ Resource type } & \multirow[b]{2}{*}{ ID } & \multirow[b]{2}{*}{ Title, author } & \multirow[b]{2}{*}{ Yr } & \multicolumn{2}{|c|}{ Subtopics } \\
\hline & & & & E. $1^{*}$ & E.2 \\
\hline \multirow[t]{3}{*}{$\begin{array}{l}\text { Book/monograph, } \\
\text { chapter }\end{array}$} & 90 & $\begin{array}{l}\text { Approaches for occupational dermal exposure assessment } \\
\text { and management, Fehrenbacher MC }\end{array}$ & 2003 & $\checkmark$ & \\
\hline & 171 & Health risk assessment, Paustenbach D & 1999 & $\checkmark$ & $\checkmark$ \\
\hline & 185 & Dermal exposure assessments, Sahmel J & 2006 & $\checkmark$ & $\checkmark$ \\
\hline $\begin{array}{l}\text { Book/monograph, } \\
\text { whole }\end{array}$ & 229 & Dermatotoxicology, Zhai H & 2004 & $\checkmark$ & \\
\hline Brochure, pamphlet & 60 & $\begin{array}{l}\text { A safety and health practitioner's guide to skin protection, } \\
\text { CPWR }\end{array}$ & 2000 & $\checkmark$ & \\
\hline
\end{tabular}

\footnotetext{
${ }^{\star}$ See footnotes at end of table.
} 


\section{PROFESSIONAL AUDIENCE Table 4E (Continued). Risk assessment}

\begin{tabular}{|c|c|c|c|c|c|}
\hline \multirow[b]{2}{*}{ Resource type } & \multirow[b]{2}{*}{ ID } & \multirow[b]{2}{*}{ Title, author } & \multirow[b]{2}{*}{ Yr } & \multicolumn{2}{|c|}{ Subtopics } \\
\hline & & & & E. $1^{*}$ & E.2 \\
\hline \multirow[t]{4}{*}{$\begin{array}{l}\text { Journal article- } \\
\text { primary }\end{array}$} & 33 & $\begin{array}{l}\text { A structured strategy for assessing chemical risks, suitable for } \\
\text { small and medium-sized enterprises, Balsat A }\end{array}$ & 2003 & $\checkmark$ & $\checkmark$ \\
\hline & 96 & $\begin{array}{l}\text { An overview of human exposure modeling activities at the } \\
\text { USEPA National Exposure Research Laboratory, Furtaw EJ Jr }\end{array}$ & 2001 & & $\checkmark$ \\
\hline & 131 & $\begin{array}{l}\text { Skin exposure to aliphatic polyisocyanates in the auto body re- } \\
\text { pair and refinishing industry: A qualitative assessment, Liu Y }\end{array}$ & 2007 & & \\
\hline & 168 & $\begin{array}{l}\text { Risk assessment and exposure control in an occupational } \\
\text { setting, Packham CL }\end{array}$ & 1996 & $\checkmark$ & $\checkmark$ \\
\hline \multirow{9}{*}{$\begin{array}{l}\text { Journal article- } \\
\text { review, meta- } \\
\text { analysis }\end{array}$} & 81 & $\begin{array}{l}\text { Occupational contact dermatitis II: Risk assessment and } \\
\text { prognosis, Emmett EA }\end{array}$ & 2003 & $\checkmark$ & \\
\hline & 100 & $\begin{array}{l}\text { Classification of dermal exposure modifiers and assignment of } \\
\text { values for a risk assessment toolkit, Goede HA }\end{array}$ & 2003 & $\checkmark$ & \\
\hline & 106 & Misinterpretation and misuse of exposure limits, Hewett $\mathrm{P}$ & 2001 & $\checkmark$ & \\
\hline & 115 & $\begin{array}{l}\text { Dermal absorption of benzene: Implications for work prac- } \\
\text { tices and regulations, Kalnas J }\end{array}$ & 2000 & $\checkmark$ & $\checkmark$ \\
\hline & 126 & $\begin{array}{l}\text { Temporal, personal and spatial variability in dermal expo- } \\
\text { sure, Kromhout H }\end{array}$ & 2001 & & $\checkmark$ \\
\hline & 139 & $\begin{array}{l}\text { Determinants of dermal exposure relevant for exposure } \\
\text { modelling in regulatory risk assessment, Marquart J }\end{array}$ & 2003 & $\checkmark$ & \\
\hline & 143 & $\begin{array}{l}\text { Assessment of dermal absorption and penetration of compo- } \\
\text { nents of a fuel mixture (JP-8), McDougal JN }\end{array}$ & 2002 & & $\checkmark$ \\
\hline & 163 & $\begin{array}{l}\text { A toolkit for dermal risk assessment and management: an } \\
\text { overview, Oppl R }\end{array}$ & 2003 & $\checkmark$ & \\
\hline & 172 & $\begin{array}{l}\text { Assessment of dermal exposure-empirical models and } \\
\text { indicative distributions, Phillips AM }\end{array}$ & 2001 & & $\checkmark$ \\
\hline
\end{tabular}

\footnotetext{
*See footnotes at end of table.
} 


\section{PROFESSIONAL AUDIENCE Table 4E (Continued). Risk assessment}

\begin{tabular}{|c|c|c|c|c|c|}
\hline \multirow[b]{2}{*}{ Resource type } & \multirow[b]{2}{*}{ ID } & \multirow[b]{2}{*}{ Title, author } & \multirow[b]{2}{*}{ Yr } & \multicolumn{2}{|c|}{ Subtopics } \\
\hline & & & & E. $1^{\star}$ & E.2 \\
\hline \multirow{7}{*}{$\begin{array}{l}\text { Journal article- } \\
\text { review, } \\
\text { meta-analysis } \\
\text { (Continued) }\end{array}$} & 177 & $\begin{array}{l}\text { Quantitating absorption of complex chemical mixtures, } \\
\text { Riviere JE }\end{array}$ & 2004 & & $\checkmark$ \\
\hline & 182 & Conservatism in pesticide exposure assessment, Ross JH & 2000 & $\checkmark$ & \\
\hline & 187 & $\begin{array}{l}\text { Percutaneous penetration studies for risk assessment, } \\
\text { Sartorelli P }\end{array}$ & 2000 & $\sqrt{ }$ & \\
\hline & 193 & $\begin{array}{l}\text { A toolkit for dermal risk assessment: Toxicological approach } \\
\text { for hazard characterization, Schuhmacher-Wolz U }\end{array}$ & 2003 & $\checkmark$ & \\
\hline & 210 & From dermal exposure to internal dose, van de Sandt J & 2008 & & $\checkmark$ \\
\hline & 212 & $\begin{array}{l}\text { RISKOFDERM: Risk assessment of occupational dermal ex- } \\
\text { posure to chemicals. An introduction to a series of papers on } \\
\text { the development of a toolkit, van Hemmen JJ }\end{array}$ & 2003 & $\sqrt{ }$ & \\
\hline & 219 & $\begin{array}{l}\text { Deriving default dermal exposure values for use in a risk } \\
\text { assessment toolkit for small and medium-sized enterprises, } \\
\text { Warren } \mathrm{N}\end{array}$ & 2003 & $\sqrt{ }$ & \\
\hline $\begin{array}{l}\text { Technical publica- } \\
\text { tion/report }\end{array}$ & 114 & $\begin{array}{l}\text { CEFIC Workshop on methods to determine dermal perme- } \\
\text { ation for human risk assessment, IOM }\end{array}$ & 2004 & & $\checkmark$ \\
\hline Web page & 209 & $\begin{array}{l}\text { USEPA, OPPT: Exposure assessment tools and models, } \\
\text { USEPA }\end{array}$ & 2005 & $\checkmark$ & $\checkmark$ \\
\hline \multirow[t]{4}{*}{ Web site } & 71 & $\begin{array}{l}\text { The pioneer in the reduction of dermal exposure [Colormetric } \\
\text { Laboratories, Inc. Home page], Colormetric Laboratories, Inc. }\end{array}$ & 2005 & $\checkmark$ & \\
\hline & 82 & $\begin{array}{l}\text { Dermatological engineering [Web page], Enviroderm Ser- } \\
\text { vices }\end{array}$ & 2005 & $\checkmark$ & $\checkmark$ \\
\hline & 87 & USEPA [Home page], USEPA & 2005 & $\checkmark$ & $\checkmark$ \\
\hline & 105 & Skin at work [Web page], HSE & 2005 & $\checkmark$ & $\checkmark$ \\
\hline
\end{tabular}

${ }^{\star}$ See footnotes at end of table.

(Continued) 
PROFESSIONAL AUDIENCE Table 4E (Continued). Risk assessment

\begin{tabular}{|c|c|c|c|c|}
\hline \multirow[b]{2}{*}{ Resource type } & \multirow[b]{2}{*}{ ID } & \multirow[b]{2}{*}{ Title, author } & \multirow[b]{2}{*}{ Yr } & Subtopics \\
\hline & & & & E. $1^{*} \quad$ E. 2 \\
\hline \multirow[t]{2}{*}{$\begin{array}{l}\text { Web site } \\
\text { (Continued) }\end{array}$} & 109 & $\begin{array}{l}\text { Human Exposure Research Organizations Exchange } \\
\text { [HEROX] [Home page], HEROX }\end{array}$ & 2005 & $\checkmark$ \\
\hline & 162 & OSHA [Home page], OSHA & 2005 & $\checkmark$ \\
\hline
\end{tabular}

${ }^{\star}$ E. $1=$ Guidelines for Risk Assessment or Analysis; E.2=Example of Risk Assessments

\section{Topic 4F. Risk Management}

Risk management completes the process of addressing potential hazards in the workplace, using information from the process of hazard identification and characterization and then taking steps to eliminate or control the hazard by various techniques. Control techniques can include changes in the process to (1)reduce or eliminate the hazard, (2) replace a more harmful chemical with a less harmful one, (3) isolate a process to minimize worker contact with the hazards, (4) modify the source to achieve less hazardous conditions/exposures, (5) change work practices to make the task less hazardous, (6) put in place administrative controls such as worker rotation, (7) train and monitor to lower each workers exposure to the hazards, or (8) use personal protective equipment to lower the exposure to the hazard. Risk management also involves evaluating the effectiveness of controls taken. The resources contain information associated with risk management.

\section{Subtopic F. 1. Exposure Control Strategies}

These resources review strategies that can be used to control skin exposures to chemicals. The following categories of control strategies are discussed: substitution; engineering controls; work practices and administrative controls; personal protective equipment; and the effectiveness of skin management programs using barrier creams, moisturizers, cleansers, and rubs.

\section{Subtopic F.2. Protocols for Risk Management}

These resources contain protocols for risk management programs. They may include protocols for the development of exposure reduction goals, protocols for the development of approaches to achieve exposure reduction goals, and evaluation tools to demonstrate program or intervention effectiveness. 
PROFESSIONAL AUDIENCE

Table 4F. Risk management

\begin{tabular}{|c|c|c|c|c|c|}
\hline \multirow[b]{2}{*}{ Resource type } & \multirow[b]{2}{*}{ ID } & \multirow[b]{2}{*}{ Title, author } & \multirow[b]{2}{*}{ Yr } & \multicolumn{2}{|c|}{ Subtopics } \\
\hline & & & & F. $1^{\star}$ & F.2 \\
\hline \multirow[t]{2}{*}{$\begin{array}{l}\text { Book/monograph, } \\
\text { chapter }\end{array}$} & 4 & $\begin{array}{l}\text { Occupational skin exposure-absorption of chemical } \\
\text { agents and assessment of exposures, Harris R }\end{array}$ & 2000 & $\checkmark$ & \\
\hline & 40 & $\begin{array}{l}\text { CPC and the skin: Practical considerations, } \\
\text { Boeniger } \mathrm{M}\end{array}$ & 2002 & $\checkmark$ & \\
\hline \multirow[t]{2}{*}{$\begin{array}{l}\text { Book/monograph, } \\
\text { chapter }\end{array}$} & 90 & $\begin{array}{l}\text { Approaches for occupational dermal exposure assessment } \\
\text { and management, Fehrenbacher MC }\end{array}$ & 2003 & $\checkmark$ & \\
\hline & 136 & Personal protective clothing, Mansdorf SZ & 2003 & $\checkmark$ & \\
\hline \multirow{5}{*}{$\begin{array}{l}\text { Book/monograph, } \\
\text { whole }\end{array}$} & 17 & Occupational skin disease, Adams RM & 1999 & $\checkmark$ & \\
\hline & 45 & Protective gloves for occupational use, Boman A & 2005 & $\checkmark$ & \\
\hline & 117 & Handbook of occupational dermatology, Kanerva L & 2000 & $\checkmark$ & \\
\hline & 157 & $\begin{array}{l}\text { Surface and dermal monitoring for toxic exposures, Ness } \\
\text { SA }\end{array}$ & 1994 & $\checkmark$ & \\
\hline & 229 & Dermatotoxicology, Zhai H & 2004 & $\checkmark$ & \\
\hline \multirow[t]{2}{*}{ Brochure, pamphlet } & 60 & $\begin{array}{l}\text { A safety and health practitioner's guide to skin protection, } \\
\text { CPWR }\end{array}$ & 2000 & $\checkmark$ & $\checkmark$ \\
\hline & 61 & $\begin{array}{l}\text { Physicians' alert for occupational contact dermatitis } \\
\text { among construction workers, CPWR }\end{array}$ & 2001 & $\checkmark$ & \\
\hline \multirow[t]{2}{*}{ Guideline } & 47 & Guideline for hand hygiene in health-care settings, CDC & 2002 & $\checkmark$ & \\
\hline & 113 & $\begin{array}{l}\text { American national standard for hand protection selection } \\
\text { criteria, International Safety Equipment Association } \\
\text { (ISEA) }\end{array}$ & 2005 & $\checkmark$ & \\
\hline $\begin{array}{l}\text { Journal article- } \\
\text { primary }\end{array}$ & 31 & $\begin{array}{l}\text { Effect of personal hygiene on blood lead levels of workers } \\
\text { at a lead processing facility, Askin DP }\end{array}$ & 1997 & $\checkmark$ & \\
\hline
\end{tabular}

\footnotetext{
*See footnotes at end of table.
} 


\section{PROFESSIONAL AUDIENCE Table 4F (Continued). Risk management}

\begin{tabular}{|c|c|c|c|c|c|}
\hline \multirow[b]{2}{*}{ Resource type } & \multirow[b]{2}{*}{ ID } & \multirow[b]{2}{*}{ Title, author } & \multirow[b]{2}{*}{ Yr } & \multicolumn{2}{|c|}{ Subtopics } \\
\hline & & & & F. $1^{\star}$ & F.2 \\
\hline \multirow{4}{*}{$\begin{array}{l}\text { Journal article- } \\
\text { primary } \\
\text { (Continued) }\end{array}$} & 98 & $\begin{array}{l}\text { Survey assessment of worker dermal exposure and under- } \\
\text { lying behavioral determinants, Geer LA }\end{array}$ & 2007 & $\checkmark$ & \\
\hline & 123 & $\begin{array}{l}\text { Skin cleansers for occupational use: testing the skin com- } \\
\text { patibility of different formulations, Klotz A }\end{array}$ & 2003 & $\checkmark$ & \\
\hline & 131 & $\begin{array}{l}\text { Skin exposure to aliphatic polyisocyanates in the auto } \\
\text { body repair and refinishing industry: a qualitative as- } \\
\text { sessment, Liu Y }\end{array}$ & 2007 & $\checkmark$ & \\
\hline & 168 & $\begin{array}{l}\text { Risk assessment and exposure control in an occupational } \\
\text { setting, Packham CL }\end{array}$ & 1996 & $\checkmark$ & $\checkmark$ \\
\hline \multirow{9}{*}{$\begin{array}{l}\text { Journal article- } \\
\text { review, meta- } \\
\text { analysis }\end{array}$} & 20 & Skin protection programmes, Agner T & 2002 & $\checkmark$ & $\checkmark$ \\
\hline & 26 & $\begin{array}{l}\text { Cleansing without compromise: The impact of cleansers } \\
\text { on the skin barrier and the technology of mild cleans- } \\
\text { ing, Ananthapadmanabhan KP }\end{array}$ & 2004 & $\checkmark$ & \\
\hline & 30 & Occupational contact dermatitis, Antezana M & 2003 & $\checkmark$ & \\
\hline & 38 & Skin exposure to isocyanates: Reasons for concern, Bello D & 2007 & $\sqrt{ }$ & \\
\hline & 42 & $\begin{array}{l}\text { In-use testing and interpretation of chemical-resistant } \\
\text { glove performance, Boeniger MF }\end{array}$ & 2002 & $\checkmark$ & \\
\hline & 51 & $\begin{array}{l}\text { Concepts of skin protection: Considerations for the } \\
\text { evaluation and terminology of the performance of skin } \\
\text { protective equipment, Brouwer DH }\end{array}$ & 2005 & $\checkmark$ & \\
\hline & 53 & $\begin{array}{l}\text { Strategies for prevention: Occupational contact dermati- } \\
\text { tis, Brown T }\end{array}$ & 2004 & $\checkmark$ & $\checkmark$ \\
\hline & 69 & Occupational issues of irritant contact dermatitis, Chew AL & 2003 & $\checkmark$ & \\
\hline & 73 & $\begin{array}{l}\text { Pesticide-related illness among migrant farm workers in } \\
\text { the United States, Das R }\end{array}$ & 2001 & $\checkmark$ & \\
\hline
\end{tabular}

${ }^{\star}$ See footnotes at end of table. 


\section{PROFESSIONAL AUDIENCE Table 4F (Continued). Risk management}

\begin{tabular}{|c|c|c|c|c|c|}
\hline \multirow[b]{2}{*}{ Resource type } & \multirow[b]{2}{*}{ ID } & \multirow[b]{2}{*}{ Title, author } & \multirow[b]{2}{*}{ Yr } & \multicolumn{2}{|c|}{ Subtopics } \\
\hline & & & & F. $1^{\star}$ & F.2 \\
\hline $\begin{array}{l}\text { Journal article- } \\
\text { review, meta- }\end{array}$ & 78 & $\begin{array}{l}\text { The epidemiology of occupational contact dermatitis, } \\
\text { Diepgen TL }\end{array}$ & 1999 & $\checkmark$ & \\
\hline \multirow[t]{12}{*}{ (Continued) } & 79 & $\begin{array}{l}\text { Skin-conditioning products in occupational dermatology, } \\
\text { Elsner P }\end{array}$ & 2003 & $\checkmark$ & \\
\hline & 103 & Occupation-related allergies in dentistry, Hamann CP & 2005 & $\sqrt{ }$ & \\
\hline & 106 & Misinterpretation and misuse of exposure limits, Hewett $\mathrm{P}$ & 2001 & & $\checkmark$ \\
\hline & 116 & $\begin{array}{l}\text { Dermatological aspects of a successful introduction and } \\
\text { continuation of alcohol-based hand rubs for hygienic } \\
\text { hand disinfection, Kampf G }\end{array}$ & 2003 & $\checkmark$ & \\
\hline & 122 & $\begin{array}{l}\text { A critique of assumptions about selecting chemical-re- } \\
\text { sistant gloves: A case for workplace evaluation of glove } \\
\text { efficacy, Klingner TD }\end{array}$ & 2002 & $\checkmark$ & \\
\hline & 125 & $\begin{array}{l}\text { Occupational skin-protection products-a review, } \\
\text { Kresken J }\end{array}$ & 2003 & $\checkmark$ & \\
\hline & 127 & $\begin{array}{l}\text { Effectiveness of skin protection creams as a preventive } \\
\text { measure in occupational dermatitis: A critical update ac- } \\
\text { cording to criteria of evidence-based medicine, Kutting B }\end{array}$ & 2003 & $\checkmark$ & \\
\hline & 128 & Toxicity of methyl methacrylate in dentistry, Leggat PA & 2003 & $\checkmark$ & \\
\hline & 135 & Occupational contact dermatitis, Lushniak BD & 2004 & $\checkmark$ & \\
\hline & 140 & Dermal toxicity due to industrial chemicals, Mathur AK & 2002 & $\checkmark$ & \\
\hline & 146 & $\begin{array}{l}\text { Differences between the sexes with regard to work-related } \\
\text { skin disease, Meding B }\end{array}$ & 2000 & $\checkmark$ & \\
\hline & 163 & $\begin{array}{l}\text { A toolkit for dermal risk assessment and management: an } \\
\text { overview, Oppl R }\end{array}$ & 2003 & & $\checkmark$ \\
\hline
\end{tabular}

*See footnotes at end of table.

(Continued) 


\section{PROFESSIONAL AUDIENCE Table 4F (Continued). Risk management}

\begin{tabular}{|c|c|c|c|c|c|}
\hline \multirow[b]{2}{*}{ Resource type } & \multirow[b]{2}{*}{ ID } & \multirow[b]{2}{*}{ Title, author } & \multirow[b]{2}{*}{ Yr } & \multicolumn{2}{|c|}{ Subtopics } \\
\hline & & & & F. $1^{*}$ & F.2 \\
\hline $\begin{array}{l}\text { Journal article- } \\
\text { review, meta- } \\
\text { analysis }\end{array}$ & 203 & $\begin{array}{l}\text { Management of dermatitis in the rubber manufacturing } \\
\text { industry, Toeppen-Sprigg B }\end{array}$ & 1999 & $\checkmark$ & $\checkmark$ \\
\hline (Continued) & 224 & The dermal toxicity of cement, Winder C & 2002 & $\checkmark$ & \\
\hline $\begin{array}{l}\text { Other-Guideline } \\
\text { from private lab }\end{array}$ & 70 & $\begin{array}{l}\text { A guide to dermal exposure reduction, Colormetric } \\
\text { Laboratories Inc. }\end{array}$ & 1999 & $\checkmark$ & $\checkmark$ \\
\hline \multirow[t]{2}{*}{$\begin{array}{l}\text { Technical publica- } \\
\text { tion/report }\end{array}$} & 129 & $\begin{array}{l}\text { Epidemiology of skin and respiratory diseases among } \\
\text { hairdressers, Leino T }\end{array}$ & 2001 & $\checkmark$ & \\
\hline & 152 & NIOSH pocket guide to chemical hazards, NIOSH & 2004 & $\checkmark$ & \\
\hline \multirow[t]{3}{*}{ Web page } & 155 & HSDB [Web page], NLM & 2005 & $\checkmark$ & \\
\hline & 208 & $\begin{array}{l}\text { Emergency response guidebook [Web page], United } \\
\text { States Department of Transportation (USDOT) }\end{array}$ & 2004 & $\checkmark$ & \\
\hline & 220 & $\begin{array}{l}\text { Dermatitis: safety and health assessment and research for } \\
\text { prevention (SHARP) [Home page] WADLI }\end{array}$ & 2005 & $\checkmark$ & \\
\hline \multirow[t]{7}{*}{ Web site } & 16 & CPI [Web site], ACC & 2006 & $\checkmark$ & \\
\hline & 18 & ATSDR [Home page], ATSDR & 2005 & $\checkmark$ & \\
\hline & 22 & AAFP [Home page], AAFP & 2005 & $\checkmark$ & \\
\hline & 32 & ASTM International [Home page], ASTM International & 2006 & $\checkmark$ & \\
\hline & 58 & CCOHS [Home page], CCOHS & 2005 & $\checkmark$ & \\
\hline & 71 & $\begin{array}{l}\text { The pioneer in the reduction of dermal exposure [Color- } \\
\text { metric Laboratories, Inc. Home page], Colormetric } \\
\text { Laboratories, Inc. }\end{array}$ & 2005 & $\sqrt{ }$ & \\
\hline & 82 & $\begin{array}{l}\text { Dermatological engineering [Web site], Enviroderm } \\
\text { Services }\end{array}$ & 2005 & $\checkmark$ & $\checkmark$ \\
\hline
\end{tabular}

*See footnotes at end of table.

(Continued) 
PROFESSIONAL AUDIENCE Table 4F (Continued). Risk management

\begin{tabular}{|c|c|c|c|c|c|}
\hline \multirow[b]{2}{*}{ Resource type } & \multirow[b]{2}{*}{ ID } & \multirow[b]{2}{*}{ Title, author } & \multirow[b]{2}{*}{ Yr } & \multicolumn{2}{|c|}{ Subtopics } \\
\hline & & & & $\mathrm{F} .1^{*}$ & F. 2 \\
\hline \multirow{7}{*}{$\begin{array}{l}\text { Web site } \\
\text { (Continued) }\end{array}$} & 87 & USEPA [Home page], USEPA & 2005 & $\checkmark$ & \\
\hline & 105 & Skin at work [Web site], HSE & 2005 & $\checkmark$ & \\
\hline & 112 & ILO [Home page], ILO & 2005 & $\checkmark$ & \\
\hline & 153 & NIOSH [Home page], NIOSH & 2005 & $\checkmark$ & \\
\hline & 154 & $\begin{array}{l}\text { TOXNET_Databases on toxicology, hazardous chemi- } \\
\text { cals, environmental health, and toxic releases [Home } \\
\text { page], NLM }\end{array}$ & 2005 & $\checkmark$ & \\
\hline & 162 & OSHA [Home page], OSHA & 2005 & $\checkmark$ & \\
\hline & 164 & OWIIPP [Web site], ORDHS & 2005 & $\checkmark$ & \\
\hline
\end{tabular}

${ }^{\star}$ F.1=Exposure Control Strategies; F.2=Risk Assessment Protocols 


\section{CHAPTER 5 \\ Overall Information Availability}

\subsection{Evaluation of Information Gaps}

The Indexed Dermal Bibliography is a collection of information resources for the anticipation, recognition, evaluation, and control of occupational skin exposures to chemicals. It is not intended to provide an exhaustive list of all publications. Rather, it includes books, review papers, regulations, and databases available to the public and credible information available on the internet. The search for was limited to resources produced from 1995 and beyond and to those dealing specifically with occupational exposures to chemicals. None of the information sources was evaluated for the accuracy of the information presented.

Within the limits of this effort, some trends were seen in the availability of resources for each topic and subtopic. Additional primary research articles produced in 1995 or later and review articles or resources produced before 1995 may be available that were not considered in this evaluation.

Overall, several topic areas appeared to have limited information. These include:

- Information on conducting risk assessments for dermal exposure.

- Guidance on the interpretation of quantitative exposure assessments and on DOELs.

- Useable information on the effectiveness of dermal exposure control measures.

- Biological monitoring performance to assess the contribution of dermal exposure to overall exposures.

- Reviews of chemical mixtures and how different combinations of chemicals can affect exposures and health effects, as well as how to assess and manage exposures to chemical mixtures.

- Protocols for evaluating biological responses to mixtures.

- Reviews and guidance documents on assessing, both qualitatively and quantitatively, intervention effectiveness and intervention design.

- Reviews and guidance documents on how to implement control measures and on how to evaluate the effectiveness of control measures for dermal exposures.

- Brochures or other educational materials that address specific chemicals that are known skin hazards. 
- Risk management strategies for particular chemicals or occupations and tasks. Construction and working with cement is an exception to this (see the eLCOSH Web site) and serves as occupations and particular chemical exposure types.

- Protocols and checklists for risk management, risk characterization, and surveillance.

- Exposure control plans designed specifically to prevent dermal hazards.

Below is a list of topics and subtopics for each audience for which few resources were identified.

\section{General Audience}

- "Specific chemicals." Information on specific chemicals was limited for the general audience, and this type of information is usually found in the professional audience references.

- There were few resources with quantitative descriptions on how exposure intensity and frequency influence exposure conditions.

- There were few guidance documents written for non-experts specific to skin hazards for qualitatively assessing skin exposure (e.g., protocols or checklists to characterize exposure when exposure data are not available, checklists and other tools for identifying exposures to hazardous chemicals, and checklists and other tools for identifying workplace conditions that contribute to skin exposure).

- There were few protocols and checklists specific to skin hazard exposure to use in identifying skin hazards at the workplace.

- There were few protocols and checklists for qualitatively identifying risks from skin exposure.

- There were few protocols and checklists for qualitatively monitoring potential skin exposures.

\section{Professional Audience}

- There are few checklists and questionnaires for quantifying skin exposure incidences.

- There is little available on how to assess the contribution of dermal exposures to overall exposures.

- More examples are needed of risk assessment for chemical mixtures. 
- Resources are needed on how to develop a dermal exposure reduction goal based on the findings, both qualitative and quantitative, of a risk assessment.

- More guidance is needed on how to develop risk management and control measures in order to achieve dermal exposure reduction goals that consider the industrial process or tasks, regulatory requirements or guidance, or experiences in similar exposure situations.

- More resources are needed for the design, selection, and implementation of evaluation methods in order to demonstrate the effectiveness of intervention approaches for exposure control.

\subsection{Future Research}

Future research should consider the gaps presented above, and include (but not limited to):

\section{General Audience}

- Materials describing the concepts of exposure intensity and frequency with respect to skin hazards.

- Protocols or checklists for identifying skin hazards and qualitatively identifying risks and monitoring for skin exposures.

\section{Professional Audience}

- Development of educational materials on skin exposures and hazards for use in instruction and hazard communication.

- Development of guidance documents and examples of risk assessment of dermal hazards.

- Development of risk management approaches and controls for dermal exposure.

- Development of methods to evaluate the effectiveness of dermal hazard intervention approaches. 



\section{APPENDIX A Full Resource Citations and Summaries}

This appendix contains the full citations and summary information for all the resources referenced in the Indexed Dermal Bibliography, listed numerically by the assigned ID number. 
Article ID:

Citation:

Resource type:

Educational materials:

Number of references:

Industries/occupations:

Specific process:

Chemical:

Specific chemicals:

Mixtures:

Audience:

Topics addressed:
1

Agency for Toxic Substance and Disease Registry (ATSDR)

[1996]. Skin lesions and environmental exposures: an overview for the occupational health nurse. ATSDR.

AAOHN J 44(11): 529-540.

Journal article—review, meta-analysis

No

0
No

Professional

A Overview

A.2 Health hazards resulting from skin exposure to chemicals

A.4 Skin physiology and function as barriers to chemical insults

B Surveillance and clinical aspects

B.4 Clinical protocols for recognition of skin exposure health effects

C Exposure Characterization

C.4 Direct methods to measure exposure

C.4.B Skin

D Hazard identification

D.1 Potential health effects resulting from specific chemicals

D.1.A Irritant contact dermatitis

D.1.B Allergic contact dermatitis/sensitization

D.1.C Systemic toxicity

D.1.D Other health effects

This paper presents a detailed discussion of pathophysiology, etiologies, diagnosis, and treatment for seven skin conditions associated with environmental exposures: irritant contact dermatitis, allergic contact dermatitis, photosensitivity contact dermatitis, chloracne, pigment alterations, contact urticaria, and malignant neoplasms. There is also a discussion of a few diagnostic procedures including patch testing, photopatch testing, and skin biopsy. 
Article ID:

Citation:

Resource type:

Educational materials:

Number of references:

Industries/occupations:

Specific process:

Chemical:

Specific chemicals:

Mixtures:

Audience:

Topics addressed:

Summary:

\section{2}

United States Environmental Protection Agency (USEPA), Office of Prevention, Pesticides and Toxic Substances (OPPTS) [1998]. Health effects test guidelines: OPPTS 870.2500 acute dermal irritation. Washington, DC: USEPA, OPPTS.

Guideline

No

14
No

Professional

D Hazard identification

D.3 Characterization protocols

D.3.A Corrosivity

D.3.B Irritation potential

This guideline specifies a procedure for testing acute dermal irritation of pesticides on animals. It is intended to meet testing requirements of both the Federal Insecticide, Fungicide, and Rodenticide Act (FIFRA) (7 U.S.C. 136, et seq.) and the Toxic Substances Control Act (TSCA) (15 U.S.C. 2601). The source materials used in developing this harmonized OPPTS test guideline are 40 CFR 798.4470 Primary Dermal Irritation; OPP 81-5 Primary Dermal Irritation (Pesticide Assessment Guidelines, Subdivision F-Hazard Evaluation; Human and Domestic Animals) USEPA report 540/09-82-025, 1982; and OECD 404 Acute Dermal Irritation/ Corrosion.
Article ID:

Citation:

Resource type:

Educational materials:
3

National Institute for Occupational Safety and Health (NIOSH) [1998]. What you need to know about occupational exposure to metalworking fluids. Cincinnati, OH: U.S. Department of Health and Human Services (DHHS), Public Health Service (PHS), Centers for Disease Control and Prevention (CDC), NIOSH, DHHS (NIOSH) Publication No. 98-116.

Guideline

Yes 
Number of references:

Industries/occupations:

Specific process:

Chemical:

Specific chemicals:

Mixtures:

Audience:

Topics addressed:

Summary:

Article ID:

Citation:

Resource type:

Educational materials:

Number of references:

Industries/occupations:
96

Heavy metals/inorganic compounds, petroleum products \& lubricants

No

General

A Overview

A.2 Health hazards resulting from skin exposure to chemicals

B Exposure characterization

B.2 Factors that influence exposure conditions

B.2.B Exposure controls

E Risk management

E.3 "Best practices"/guidelines/recommendations

E.3.B Engineering controls

E.3.C Work practice/administration controls

E.3.D PPE and PPE rules

E.3.E Skin management, barrier creams, moisturizers, cleansers, and rubs

This document summarizes the findings of the NIOSH Criteria Document: NIOSH Criteria for a Recommended Standard: Occupational Exposure to Metalworking Fluids (NIOSH Publication Number 98-102). It also provides a critical review of the scientific and technical information available on the subject as well as a scientific basis for the recommendations. It is an educational document intended to communicate basic information.

\section{4}

Harris R, ed. [2000]. Occupational skin exposureabsorption of chemical agents and assessment of exposures. In: Patty's Industrial Hygiene, 5th ed. Vol. I, Recognition and evaluation of chemical agents. Indianapolis, IN: John Wiley \& Sons.

Book/monograph, chapter

No

142 


\section{Specific process:}

Chemical:

Specific chemicals:

Mixtures:

Audience:

Topics addressed:

Summary:
No

Professional

A Overview

A.4 Skin physiology and function as barriers to chemical insults

C Exposure characterization

C.2 Description of factors influencing exposure conditions

C.2.E Uptake

D Hazard identification

D.1 Potential health effects resulting from specific chemicals

D.1.A Irritant contact dermatitis

D.1.B Allergic contact dermatitis/sensitization

F Risk management

F.1 Exposure control strategies

F.1.A Substitution

F.1.B Engineering controls

F.1.C Work practice/Administrative controls

F.1.D PPE and PPE rules

F.1.E Skin management, barrier creams, moisturizers, cleansers, and rubs

This chapter from Patty's Industrial Hygiene Volume 1 includes a discussion of factors that cause and contribute to occupational dermatoses, covering chemicals, primary irritants, allergic contact dermatitis, plants and wood, photosensitivity, mechanical, physical, and biological factors. The chapter also discusses the physiology of the skin, patch tests, prevention, and control.
Article ID:

\section{Citation:}

Resource type:

Educational materials:
5

Keil CB, ed. [2000]. Dermal exposure modeling. In:

Mathematical models for estimating occupational exposure to chemicals. Fairfax, VA: American Industrial Hygiene Association (AIHA).

Book/monograph, chapter

No 
Number of references:

Industries/occupations:

Specific process:

Chemical:

Specific chemicals:

Mixtures:

Audience:

Topics addressed:
30

No

Professional

C Exposure characterization

C.2 Description of factors influencing exposure conditions

C.2.B Exposure concentration

C.2.C Skin area affected

C.2.E Uptake

C.5 Exposure modeling

This chapter of the book focuses on estimating dermal exposures. There is a discussion of absorption mechanics, absorption factors, modeling, and data gaps and suggestions for additional research.
Article ID:

Citation:

Resource type:

Educational materials:

Number of references:

Industries/occupations:

Specific process:

Chemical:

Specific chemicals:

Mixtures:

Audience:

Topics addressed:

Summary:

\section{6}

Health and Safety Executive (HSE) [2000]. Selecting protective gloves for work with chemicals. Sudbury, UK: HSE Books.

Brochure, pamphlet

Yes

3

General-overview

No

General

E Risk management

E.3 "Best practices"/guidelines/recommendations

E.3.D PPE and PPE rules

This leaflet, for employers and the self-employed, provides basic advice on selecting gloves to protect the wearer from chemical agents. It discusses UK law, chemical resistance of protective gloves, and selection of gloves. A PDF version of this leaflet is available on their Web site [www.hse.gov.uk/]. 
Article ID: $\quad 7$

Citation:

Resource type:

Educational materials:

Number of references:

Industries/occupations:

Specific process:

Chemical:

Specific chemicals:

Mixtures:

Audience:

Topics addressed:

Summary:
Occupational Health Department [2000]. Did you know

about the health hazards of benzene? Singapore: Republic of Singapore, Ministry of Manpower, Occupational Health Department.

Brochure, pamphlet

Yes

0

Solvents

Benzene

No

General

A Overview

A.1 Occurrence of skin exposures in the workplace

A.2 Health hazards resulting from skin exposure to chemicals

C Hazard identification

C.1 Risk phrases, hazard symbols, skin designations

E Risk management

E.3 "Best practices"/guidelines/recommendations

E.3.A Substitution

E.3.B Engineering controls

E.3.C Work practice/administration controls

E.3.D PPE and PPE rules

This brochure from the Singapore Department of Industrial Health presents the hazards of benzene. It describes properties, main uses, exposure hazards, acute effects (narcotic effect, drying effect on skin and mucous membranes), chronic effects (anemia, leukemia), technical control measures (substitution, engineering controls, personal protection), and medical control measures (preemployment examinations, periodic medical examinations).

\section{8}

Occupational Health Department [2000]. Did you know the hazards of solvents? Singapore: Republic of Singapore, Ministry of Manpower, Occupational Health Department. 
Resource type:

Educational materials:

Number of references:

Industries/occupations:

Specific process:

Chemical:

Specific chemicals:

Mixtures:

Audience:

Topics addressed:

Summary:
Brochure, pamphlet

Yes

0

Solvents

No

General

A Overview

A.1 Occurrence of skin exposures in the workplace

A.2 Health hazards resulting from skin exposure to chemicals

E Risk management

E.3 "Best practices"/guidelines/recommendations

E.3.B Engineering controls

E.3.C Work practice/administration controls

This leaflet from the Singapore Department of Industrial Health presents the hazards of solvents. It describes where solvents are used, why solvents are hazardous (volatility, flammability, explosivity, reactivity), their acute health effects (irritation of eyes, nose and throat, headache, nausea, poor coordination, arrhythmia), and their chronic health effects (skin dryness, allergic reactions, neurobehavioural changes, liver damage, paralysis, leukaemia). Other topics include storage and handling, limitation of exposure, safe working methods, medical supervision, and first-aid measures.

\section{9}

HSE [2001]. Assessing and managing risks at work from skin exposure to chemical agents: guidance for employers and health and safety specialists. Sudbury, UK: HSE Books. Brochure, pamphlet

Yes

10

General-overview

Lists occupational groups of concern including hairdressers.

General-overview 
Specific chemicals:

Mixtures:

Audience:

Topics addressed:

Summary: methylene bis (discussed briefly)

No

General

A Overview

A.1 Occurrence of skin exposures in the workplace

A.2 Health hazards resulting from skin exposure to chemicals

A.3 Dermal regulations and skin notations

B Exposure characterization

B.1 Job/tasks, industries/processes, or chemicals associated with skin exposures

C Hazard identification

C.3 Protocols/checklists to identify skin hazards in the workplace

E Risk management

E.3 "Best practices"/guidelines/recommendations

E.3.A Substitution

E.3.B Engineering controls

E.3.C Work practice/administration controls

E.3.D PPE and PPE rules

This guidance from the UK provides practical advice for employers and the self-employed to reduce the risk to skin exposures from chemicals. The guidance explains how health effects can be caused by skin exposure to chemicals, provides examples of chemicals known to cause health effects, and offers advice for assessing and reducing the risk of skin exposures.
Article ID:

Citation:

Resource type:

Educational materials:

Number of references:

Industries/occupations:

Specific process:

Chemical:

\section{0}

USEPA [2001]. Risk assessment guidance for superfund

(RAGS), Vol. I: Human health evaluation manual (Part

$\mathrm{E}$, supplemental guidance for dermal risk assessment).

Washington, DC: USEPA.

Technical publication/report

No

93

Hazardous waste management 
Specific chemicals:

Mixtures:

Audience:

Topics addressed:

Summary:
Contains permeability coefficients for 28 inorganic compounds including 12 chromium compounds

No

Professional

C Exposure characterization

C.2 Description of factors influencing exposure conditions

C.2.A Exposure intensity/frequency/duration

C.2.B Exposure concentration

C.2.C Skin area affected

C.2.E Uptake

C.5 Exposure modeling

The supplemental guidance section (Part E) to the Risk Assessment Guidance for Superfund(RAGS), Volume I: Human Health Evaluation Manual incorporates and updates the principles of the USEPA interim report, Dermal Exposure Assessment: Principles and Applications, released in 1992. Part E contains methods for conducting dermal risk assessments. Chapters include introduction and flowchart, hazard identification, exposure assessment, toxicity assessment, risk characterization, and conclusion/ recommendations.
Article ID:

Citation:

Resource type:

Educational materials:

Number of references:

Industries/occupations:

Specific process:

Chemical:

Specific chemicals:

Mixtures:

Audience:

Topics addressed:

Summary:

\section{1}

HSE [2001]. Cost and effectiveness of chemical protective gloves for the workplace. Sudbury, UK: HSE Books.

Brochure, pamphlet

Yes

8

No

General

E Risk management

E.3 "Best practices"/guidelines/recommendations

E.3.D PPE and PPE rules

This UK publication provides employers with advice on the cost and effectiveness of gloves and personal protective 
equipment (PPE) for those industries where employees incur risk from dermal exposure to chemicals. It is available for purchase on the HSE Web site [www.hse.gov. $\mathrm{uk} /]$.

Article ID:

\section{Citation:}

Resource type:

Educational materials:

Number of references:

Industries/occupations:

Specific process:

Chemical:

Specific chemicals:

Mixtures:

Audience:

Topics addressed:

Summary:

\section{2}

HSE [2001]. Choice of skin care products for the workplace. Sudbury, UK: HSE Books.

Brochure, pamphlet

Yes

0
No

General

A Overview

A.2 Health hazards resulting from skin exposure to chemicals

E Risk management

E.3 "Best practices"/guidelines/recommendations

E.3.E Skin management, barrier creams, moisturizers, cleansers, and rubs

This UK publication provides employers with information on skin care products, their selection, and how they fit into an overall skin care program for those industries where employees incur risk from dermal exposure to chemicals. This is available for purchase on the HSE Web site [www.hse.gov.uk/].
Article ID:

Citation:

Resource type:

Educational materials:

Number of references:

Industries/occupations:

Specific process:

\section{3}

American Skin Association (ASA) [2005]. [www.americanskin.org/frameset.htm].

Web site

Yes

General-overview 
Chemical:

Specific chemicals:

Mixtures:

Audience:

Topics addressed:

Summary:
General—overview, latex

No

General

A Overview

A.1 Occurrence of skin exposures in the workplace

A.2 Health hazards resulting from skin exposure to chemicals

The ASA is a volunteer-led health organization that works on issues associated with skin disorders. One of the primary missions of the ASA is to raise public awareness of the wide range of skin disorders through ongoing public education. They produce a consumer-oriented quarterly newsletter called Skin Facts. Past issues can be accessed through the Web site's archives. Past articles have included skin disease in the workplace and latex sensitivity.
Article ID:

Citation:

Resource type:

Educational materials:

Number of references:

Industries/occupations:

Specific process:

Chemical:

Specific chemicals:

Mixtures:

Audience:

Topics addressed:

\section{4}

Montana Department of Labor and Industries (MTDLI) Employment Relations [2005]. [http://erd.dli.mt.gov/].

Web site

No

General-overview

General-overview, latex

No

General

A Overview

A.1 Occurrence of skin exposures in the workplace

A.2 Health hazards resulting from skin exposure to chemicals

B Exposure characterization

B.1 Job/tasks, industries/processes, or chemicals associated with skin exposures

E Risk management

E.1 Overview of skin exposure control options

E.3 "Best practices"/guidelines/recommendations 


\section{E.3.A Substitution \\ E.3.B Engineering controls \\ E.3.C Work practice/administration controls \\ E.3.D PPE and PPE rules \\ E.3.E Skin management, barrier creams, moisturizers, cleansers, and rubs \\ E.4 Guidelines/recommendations for postexposure skin decontamination}

Summary:

The MT DLI Safety and Health Bureau is responsible for workplace safety and health through inspection, consultation, technical assistance, and training. Over 100 different occupational safety and health documents and brochures are available either electronically through their Web site or hard copies can be ordered through their Web site. Documents are accessed through the program samples topic on the Health and Safety Bureau's drop-down box. Dermal exposure-related documents and brochures found at this site include:

- Dermatitis Prevention: Occupational Skin Disorders

- Latex Allergy

- Job Safety Analysis Packet (though generic, can be used to evaluate dermal hazards)

Article ID:

15

Citation:

Resource type:

Educational materials:

Number of references:

Industries/occupations:

Specific process:

Chemical:

Specific chemicals:

Mixtures:

Audience:

Topics addressed:
1105 Media, Inc. [2006]. Occupational health and safety. [www.ohsonline.com].

Web site

No

General—overview, Medical Services

General—overview, heavy metals/inorganic compounds, latex, plastics, resins

Hexavalent chromium (CrVI)

No

General

A Overview

A.1 Occurrence of skin exposures in the workplace

A.3 Dermal regulations and skin notations

B Exposure characterization 
Summary:

Article ID:

\section{Citation:}

Resource type:

Educational materials:

Number of references:

Industries/occupations:

Specific process:

Chemical:

Specific chemicals:

Mixtures:

Audience:

Topics addressed:
B.1 Job/tasks, industries/processes, or chemicals associated with skin exposures

B.3 Protocols/checklists to characterize exposure to skin hazards

C Hazard identification

C.1 Risk phrases, hazard symbols, skin designations

C.3 Protocols/checklists to identify skin hazards in the workplace

E Risk management

E.2 Protocols/checklists to monitor potential exposures

E.3 "Best practices"/guidelines/recommendations

E.3.C Work practice/administration controls

E.3.D PPE and PPE rules

E.3.E Skin management, barrier creams, moisturizers, cleansers, and rubs

The Occupational Health and Safety online magazine periodically features articles on dermal exposure and control. They have archived past issues that can be searched by subject. Dermal exposure articles that can be found online include:

- "Effective Dermal Protection"

- "Butyl \& Viton Hand Protection"

- "Latex Allergy \& Dermatitis"

- "CrVI: New Regulations and Detection Methods"

16

American Chemistry Council (ACC) [2006]. Center for the Polyurethanes Industry (CPI). [www.polyurethane.org/].

Web site

Yes

Manufacturing-Chemical

Plastics and resins

Diisocyanates

No

General

A Overview 


\begin{tabular}{|c|c|c|}
\hline & A.1 & Occurrence of skin exposures in the workplace \\
\hline & A. 2 & $\begin{array}{l}\text { Health hazards resulting from skin exposure to } \\
\text { chemicals }\end{array}$ \\
\hline & $\mathrm{B}$ & Exposure characterization \\
\hline & B.1 & $\begin{array}{l}\text { Job/tasks, industries/processes, or chemicals } \\
\text { associated with skin exposures }\end{array}$ \\
\hline & B.2 & Factors that influence exposure conditions \\
\hline & B.2.A & Exposure intensity/frequency \\
\hline & B.2.B & Exposure controls \\
\hline & $\mathrm{E}$ & Risk management \\
\hline & E.1 & Overview of skin exposure control options \\
\hline & E.3 & "Best practices"/guidelines/recommendations \\
\hline & E.3.A & Substitution \\
\hline & E.3.B & Engineering controls \\
\hline & E.3.C & Work practice/administration controls \\
\hline & E.3.D & PPE and PPE rules \\
\hline & E.4 & $\begin{array}{l}\text { Guidelines/recommendations for postexposure } \\
\text { skin decontamination }\end{array}$ \\
\hline Summary: & $\begin{array}{l}\text { The C } \\
\text { CPI's } \\
\text { chemi } \\
\text { polyur } \\
\text { inforn } \\
\text { interes }\end{array}$ & $\begin{array}{l}\text { is a business unit of the American Plastics Council. } \\
\text { members include U.S. producers or distributors of } \\
\text { s and equipment used to make polyurethane and } \\
\text { ane product manufacturers. Their Web site contains } \\
\text { ion on polyurethane health and safety. Resources of } \\
\text { ssociated with dermal exposure issues include: }\end{array}$ \\
\hline & $\cdot 1$ & $\begin{array}{l}\text { per-reactivity and Other Health Effects of } \\
\text { socyanates: Guidelines for Medical Personnel }\end{array}$ \\
\hline & - & rking with TDI: What you should know \\
\hline & - 1 & yol Resin Blends Safety and Handling Guidelines \\
\hline & - & rking with MDI: What you should know \\
\hline Article ID: & 17 & \\
\hline Citation: & $\begin{array}{l}\text { Adam } \\
\text { Philad }\end{array}$ & $\begin{array}{l}\text { M [1999]. Occupational skin disease, 3rd ed. } \\
\text { hia: Saunders. }\end{array}$ \\
\hline Resource type: & Book/ & nograph, whole \\
\hline Educational materials: & No & \\
\hline Number of references: & 5112 & \\
\hline Industries/occupations: & Gener & -overview, Agricultural, Beauty/Cosmetology, \\
\hline & $\begin{array}{l}\text { Clean } \\
\text { Manu }\end{array}$ & $\begin{array}{l}\text { /Janitorial/Maid, Construction, Forestry/Fisheries, } \\
\text { turing_Chemical, Manufacturing-Other, }\end{array}$ \\
\hline
\end{tabular}




\section{Specific process:}

\section{Chemical:}

Specific chemicals:

Mixtures:

Audience:

Topics addressed:
Medical Services, Service-Food, Service-Medical, Service-Other, Transportation/Communications/Utility

Describes the occupation and risks, lists irritants, standard allergens and additional allergens for the following occupations:

Air hammer operators, abattoir workers, aircraft workers, anodizers, artists, asphalt workers, athletes, automobile mechanics, bakers, barbers, bartenders, bath attendants, battery makers, beekeepers, blueprint makers, bookbinders, brake lining workers, butchers, poultry processors, cabinet makers, candle makers, cannery workers, carpenters, cashiers, caulkers, cement workers, ceramic workers, chemists, cigarette and cigar makers, construction workers, cosmetologists, dairy workers, dentists and dental personnel, dry cleaners, electricians, electron microscopy workers, electroplaters, embalmers, engravers, firefighters, floor layers, florists, food service workers, forest workers and loggers and foresters, foundry workers, fur processors, glaziers, healthcare workers, highway construction workers, histology technicians, house workers, insulation workers, jewelers, laundry workers, locksmiths, machinists, medical personnel, metal polishers, musicians, office workers, optical technicians, painters and paperhangers, papermakers, performing artists, pest control workers, pharmacists, photographers, plastics assemblers and fabricators, plumbers and pipe fitters, police officers and detectives, postal workers, pottery and porcelain makers, printers, radio and television repairers, railroad shop workers, roofers, semiconductor and electronics workers, sheet-metal workers, shoe repairers, silk-screening workers, solderers and brazers, stonemasons, swimming pool personnel, tannery workers, tattoo artists, taxidermists, textile workers, theatrical artists, tile setters, tobacco workers, veterinarians, welders, wine makers, and wire drawing operators.

General-overview, heavy metals/inorganic compounds, pesticides, petroleum products \& lubricants, plastics and resins, rubber additives, soaps and detergents, solvents, other: semiconductors, plants, steroids, paints

Yes

Professional

C Exposure characterization

C.2 Description of factors influencing exposure conditions

C.2.A Exposure intensity/frequency/duration

C.2.B Exposure concentration 


\begin{tabular}{|c|c|}
\hline C.2.C & Skin area affected \\
\hline C.2.E & Uptake \\
\hline C. 4 & Direct methods to measure exposure \\
\hline C.4.A & Surfaces \\
\hline C.4.B & Skin \\
\hline C.4.C & Biomonitoring \\
\hline C. 5 & Exposure modeling \\
\hline $\mathrm{D}$ & Hazard identification \\
\hline D.1 & $\begin{array}{l}\text { Potential health effects resulting from specific } \\
\text { chemicals }\end{array}$ \\
\hline D.1.A & Irritant contact dermatitis \\
\hline D.1.B & Allergic contact dermatitis/sensitization \\
\hline D.1.C & Systemic toxicity \\
\hline D.1.D & Other health effects \\
\hline D.3 & Characterization protocols \\
\hline D.3.E & $\begin{array}{l}\text { Measurement of skin permeation rates and } \\
\text { reservoir effects }\end{array}$ \\
\hline $\mathrm{F}$ & Risk management \\
\hline F.1 & Exposure control strategies \\
\hline F.1.E & $\begin{array}{l}\text { Skin management, barrier creams, moisturizers, } \\
\text { cleansers, and rubs }\end{array}$ \\
\hline
\end{tabular}

Summary:

This comprehensive book is a standard reference on occupational skin diseases. It provides an overview as well as an in-depth discussion of skin diseases associated with dozens of specific occupations, their causes, and health effects. Each chapter was written by national and international researchers and is individually referenced. It includes a step-by-step guide for making precise diagnoses, considerations for differential diagnoses, and practical solutions for skin disease problems.

CHAPTER HEADINGS: Irritants/Allergic Contact Dermatitis:

General Principles and Causes/Physical Causes of Occupational Skin Disease/Systemic Toxicity from Percutaneous Absorption/Biological Causes/Contact Urticaria Due to Occupational Exposure/Acne and Folliculitis Caused By Mechanical Factors "Chloracne"/ Occupational Skin Cancer/Occupational Nail Disorders/ Phototoxicity and Photosensitivity Reactions / Occupational Nail Disorders/Diagnosis and Differential Diagnosis/Atopy, Atopic Dermatitis and Occupational Skin Disease/Diagnostic Patch Testing/The Computer in Occupational Skin Disease/Multiple Chemical Sensitivities/ 
Prevention, Rehabilitation, Treatment/Health Risk Assessment and Occupational Dermatology/Workers Compensation/Plant Inspection/Industrial Processes Commonly Associated with Skin Disease/Soaps and Detergents/Cosmetics/Corticosteroids/Metals/Plastics and Platicizers/Semiconductor Industry/Paints, Varnishes and Lacquers/Solvents/Occupational Skin Problems from Natural and Synthetic Rubber/Petroleum and Petroleum Derivatives/Occupational Dermatitis from Plants and Woods/Pesticides and Other Agriculture Chemicals/Job Descriptions with Their Irritants and Allergens.

Article ID: 18

Citation:

Resource type:

Educational materials:

Number of references:

Industries/occupations:

Specific process:

Chemical:

Specific chemicals:

Mixtures:

Audience:

Topics addressed:

\section{ATSDR [2008]. [www.atsdr.cdc.gov/].}

Web site

No

General-overview

Abrasives, cleaning agents, coolants, corrosives, fiberglass and other fibers, heavy metals/inorganic compounds, nanoparticles, organic dyes, particulates, pesticides, petroleum products \& lubricants, PAHs, PCBs, rubber additives, solvents

Information on hundreds of chemicals

No

Professional

A Overview

A.1 Occurrence of skin exposures in workplace

B Surveillance and clinical aspects

B.4 Clinical protocols for recognition of skin exposure health effects

D Hazard identification

D.1 Potential health effects resulting from specific chemicals

D.1.A Irritant contact dermatitis

D.1.B Allergic contact dermatitis/sensitization

D.1.C Systemic toxicity

D.1.D Other health effects

D.1.E Contribution to overall exposure 
D.2 Summaries of health effects, dose-response relationships

F Risk management

F.1 Exposure control strategies

F.1.C Work practice/Administrative controls

F.1.D PPE and PPE rules

Summary:

The ATSDR is a federal public health agency of the U.S. DHHS. ATSDR is directed by a congressional mandate to perform specific functions concerning the effect on public health of hazardous substances in the environment. These functions include response to emergency releases of hazardous substances, information development and dissemination, and education and training concerning hazardous substances. The Web site contains a number of resources applicable to occupational dermal exposure to chemicals, including:

- Medical management guidelines (MMGs): Guidelines for acute chemical exposures were developed by ATSDR to aid emergency department physicians and other emergency healthcare professionals who manage acute exposures resulting from chemical incidents. Information provided in the guidelines includes potential routes of exposure, applicable exposure standards and guidelines, health effects, and protective measures to be taken by rescue workers, decontamination procedures and printable followup documents for patients who have been exposed to specified chemicals. Guidelines are available for approximately 50 chemicals. [http://www.atsdr.cdc. gov/MHMI/mmg.html]

- Toxicological profile information sheets: Toxicological profiles for hazardous substances found at National Priorities List (NPL) sites. Profiles are available on over 250 chemicals (see ID 19 for more details).

- ToxFAQs: A series of summaries taken from toxicological profiles and public health statements. Each fact sheet serves as a quick and easy-tounderstand guide. Answers are provided to the most frequently asked questions (FAQs) about exposure to hazardous substances found around hazardous waste sites and the effects of exposure on human health.

- Interaction profiles for toxic substances: A series of documents being developed for certain priority mixtures that are of special concern to ATSDR. The purpose of the interaction profiles is to evaluate data on the toxicology of the mixture and on the joint toxic action of the chemicals in the mixture in 
order to recommend approaches for exposure-based assessment of the potential hazard to public health.

Article ID:

Citation:

Resource type:

Educational materials:

Number of references:

Industries/occupations:

Specific process:

Chemical:

Specific chemicals:

Mixtures:

Audience:

Topics addressed:
19

ATSDR [2005]. Toxicological profile information sheet. [www.atsdr.cdc.gov/toxpro2.html].

Web page

No

General—overview

Abrasives, cleaning agents, coolants, corrosives, hand cleansers, heavy metals/inorganic compounds, organic dyes, particulates, pesticides, petroleum products \& lubricants, plastics and resins, PAHs, PCBs, rubber additives, solvents

250 chemicals listed

No

Professional

A Overview

A.2 Health hazards resulting from skin exposure to chemicals

A.3 Investigation, intervention, and control of occupational skin exposures

C Exposure characterization

C.4 Direct methods to measure exposure

C.4.C Biomonitoring

D Hazard identification

D.1 Potential health effects resulting from specific chemicals

D.1.A Irritant contact dermatitis

D.1.B Allergic contact dermatitis/sensitization

D.1.C Systemic toxicity

D.1.D Other health effects

D.1.E Contribution to overall exposure

D.2 Summaries of health effects, dose-response relationships

The ATSDR produces toxicological profile information sheets for hazardous substances found at National 
Priorities List (NPL) hazardous waste sites. Although geared toward environmental rather than occupational exposures, the sheets contain useful information for occupational settings as well. So far, 282 toxicological profiles have been published or are under development and cover more than 250 substances. Each chemical profile contains information on health effects, chemical and physical information, potential for human exposure, analytical methods, and regulations and advisories.

Each profile is written for a general audience and contains a public health statement that includes information written in nontechnical terms on what the chemical is, how one might be exposed to it, how the chemical enters and leaves the body, the effects of exposure, and medical tests to determine if a worker has been exposed.

Article ID:

\section{0}

\section{Citation:}

Resource type:

Educational materials:

Number of references:

Industries/occupations:

Specific process:

Chemical:

Specific chemicals:

Mixtures:

Audience:

Topics addressed:

Summary:
Agner T, Held E [2002]. Skin protection programmes. Contact Dermatitis 47(5):253-56.

Journal article—review, meta-analysis

No

46

No

Professional

F Risk management

F.1 Exposure control strategies

F.1.C Work practice/Administrative controls

F.1.D PPE and PPE rules

F.1.E Skin management, barrier creams, moisturizers, cleansers, and rubs

F.2 Protocols for risk management

F.2.B Development of approach to achieve exposure reduction goal

The article discusses 10 recommendations for skin protection involving washing, gloves, moisturizers, and behavioral changes. 
Article ID:

Citation:

Resource type:

Educational materials:

Number of references:

Industries/occupations:

Specific process:

Chemical:

Specific chemicals:

Mixtures:

Audience:

Topics addressed:

\section{1}

American Academy of Family Physicions (AAFP) [2004]. Skin problems: how to protect yourself from job-related skin problems. [http://familydoctor.org/750.xml].

Web page

No

General—overview

General—overview

No

General

A Overview

A.1 Occurrence of skin exposures in the workplace

A.2 Health hazards resulting from skin exposure to chemicals

E Risk management

E.1 Overview of skin exposure control options

This very general review of job-related skin problems includes a discussion on how workers can protect themselves against workplace skin hazards.
Article ID:

Citation:

Resource type:

Educational materials:

Number of references:

Industries/occupations:

Specific process:

Chemical:

Specific chemicals:

Mixtures:

Audience:

Topics addressed:

\section{2}

AAFP [2005]. [www.aafp.org]

Web site

No

General—overview

General—overview

No

General

A Overview

A.1 Occurrence of skin exposures in the workplace

A.2 Health hazards resulting from skin exposure to chemicals 
Summary:

\section{Article ID:}

\section{Citation:}

Resource type:

Educational materials:

Number of references:

Industries/occupations:

Specific process:

Chemical:
Exposure characterization

B.1 Job/tasks, industries/processes, or chemicals associated with skin exposures

C Hazard identification

C.2 Tables/charts/lists of hazards for specific chemicals

E Risk management

E.1 Overview of skin exposure control options

E.3 "Best practices"/guidelines/recommendations

E.3.D PPE and PPE rules

E.3.E Skin management, barrier creams, moisturizers, cleansers, and rubs

The AAFP is one of the largest national medical organizations, representing more than 94,000 family physicians, family medicine residents, and medical students nationwide. The Web site contains information related to dermal exposure and occupational skin disease. Key information includes:

- Skin problems on the job-patient information handsheet—This handsheet, written for a more general audience, provides a brief overview of skin hazards and what workers can do to protect themselves.

- Occupational Skin Disease-This article, written for medical professionals, provides an overview of cause, diagnosis, and control of occupational skin disease, including a more detailed description of irritant contact dermatitis and allergic contact dermatitis and occupational groups at risk.

\section{3}

American Conference of Governmental Industrial

Hygienists (ACGIH) [2001]. Documentation of the threshold limit values for chemical substances, 7 th ed. Cincinnati: ACGIH.

Guideline

No

General—overview

Abrasives, cleaning agents, coolants, corrosives, fiberglass and other fibers, heavy metals/inorganic compounds, latex, nanoparticles, organic dyes, particulates, pesticides, 
Specific chemicals:

Mixtures:

Audience:

Topics addressed:

petroleum products \& lubricants, plastics and resins, PAHs, PCBs, rubber additives, solvents

Includes over 500 chemicals

No

Professional

D Hazard identification

D.1 Potential health effects resulting from specific chemicals

D.1.A Irritant contact dermatitis

D.1.B Allergic contact dermatitis/sensitization

D.1.C Systemic toxicity

D.1.D Other health effects

D.1.E Contribution to overall exposure

D.2 Summaries of health effects, dose-response relationships

ACGIH is a scientific association with a number of technical committees that develop professional practice guidelines, such as threshold limit values (TLVs) for chemical substances and physical agents and the biological exposure indices (BEIs) for selected chemicals. The TLVs and BEIs are developed as guidelines to assist in the control of health hazards. The documentation of the TLVs and BEIs provides the basic rationale for the development of TLVs and of BEIs. The publication consists of documentation for over 750 chemical and physical agents. These recommendations or guidelines are intended for use in the practice of industrial hygiene, to be interpreted and applied only by a person trained in this discipline. They are not developed for use as legal standards and ACGIH does not advocate their use as such. The documentation is available in hard copy, on CD, or downloadable from the ACGIH Web site.

\section{4}

Citation:

Resource type:

Educational materials:

Number of references:

Industries/occupations:

Specific process:

Chemical:
AIHA [2005]. 2005 Emergency response planning guidelines ERPG and workplace environmental exposure level WEELs handbook. Fairfax, VA: AIHA.

Brochure, pamphlet

Yes

15 
Specific chemicals:

Mixtures: $\quad$ No

Audience: $\quad$ General

Topics addressed: $\quad$ A $\quad$ Overview

A.3 Dermal regulations and skin notations

Summary:

This pocket-sized emergency reference guide presents an overview of two sets of exposure limits: the AIHA ERPG (114) and WEELs (108). It contains recommended values for each series. In addition to a glossary, both ERPG and WEELs sections include background information, user guidance, value rationale, sample documents, and values. There is also an explanation on biological environmental exposure limits (BEELs).

Article ID:

25

Citation:

Resource type:

Educational materials:

Number of references:

Industries/occupations:

Specific process:

Chemical:

Specific chemicals:

Mixtures:

Audience:

Topics addressed:

Summary:
AIHA [2006]. [www.aiha.org].

Web site

No
General—overview

No

Professional

A Overview

A.5 Dermal regulations and skin notations

The AIHA is a nonprofit, international association of occupational and environmental health professionals. Among other things, the AIHA Web site is a source of information on occupational and environmental health and safety topics and publications, including dermal exposure. AIHA members can serve on a number of committees that support AIHA's mission to promote healthy and safe environments by advancing the science, principles, practice, and value of industrial hygiene and occupational and environmental health and safety. The Dermal Project Team of the Exposure Assessment Strategies Committee focuses on issues associated with dermal exposure assessment. On the Dermal Project Team Web page are resources related to dermal exposure, including a list of general sources of dermal information. 
The AIHA Workplace Environmental Exposure Levels (WEELs) Committee works on establishing and updating AIHA's WEELs. These include a skin designation for chemicals in which significant amounts may be absorbed through the skin, and therefore contribute to overall exposures.

Article ID:

Citation:

Resource type:

Educational materials:

Number of references:

Industries/occupations:

Specific process:

Chemical:

Specific chemicals:

Mixtures:

Audience:

Topics addressed:
26

Ananthapadmanabhan KP, Moore DJ, Subramanyan K, Misra M, Meyer F [2004]. Cleansing without compromise: The impact of cleansers on the skin barrier and the technology of mild cleansing. Dermatol Ther 17(1):16-25. Journal article-review, meta-analysis

No

40

Hand cleansers

No

Professional

D Hazard identification

D.1 Potential health effects resulting from specific chemicals

D.1.A Irritant contact dermatitis

F Risk management

F.1 Exposure control strategies

F.1.E Skin management, barrier creams, moisturizers, cleansers, and rubs

Summary:

This review discusses the benefits and health impacts of skin cleansers and compares different kinds of skin cleansing products.
Article ID:

Citation:

Resource type:

Educational materials:

Number of references:

\section{7}

Andersen KE [2003]. Occupational issues of allergic contact dermatitis. Int Arch Occup Environ Health 76(5):347-50.

Journal article—review, meta-analysis

No

28 
Industries/occupations:

Specific process:

Chemical:

Specific chemicals:

Mixtures:

Audience:

Topics addressed:

Summary:
No

Professional

B Surveillance and clinical aspects

B.1 Surveillance study reporting incidences of occupational skin exposures

B.1.A Skin exposure major focus

B.4 Clinical protocols for recognition of skin exposure health effects

C Exposure characterization

C.4 Direct methods to measure exposure

C.4.B Skin

C.4.C Biomonitoring

D Hazard identification

D.2 Summaries of health effects, dose-response relationships

This review addresses occupational allergic contact dermatitis. The article discusses epidemiological data, diagnosis, exposure assessment, and dose-response relationship. Preventive measures are also discussed, though in general terms.

Article ID:

\section{8}

Citation:

Resource type:

Educational materials:

Number of references:

Industries/occupations:

Specific process:

Chemical:

Specific chemicals:

Mixtures:
Andersen, KE [1999]. Systemic toxicity from percutaneous absorption. In: Adams RM, ed. Occupational skin disease. Philadelphia: Saunders, 69-85.

Book/monograph, chapter

No

147

Heavy metals/inorganic compounds, pesticides, rubber additives, solvents, phosphate esters, chlorinated hydrocarbons, topical drugs and toiletries, pharmaceuticals 
Audience:

Topics addressed:

Summary:
Professional

A Overview

A.4 Skin physiology and function as barriers to chemical insults

B Surveillance and clinical aspects

B.1 Surveillance study reporting incidences of occupational skin exposures

B.1.A Skin exposure major focus

B.1.B Skin exposure minor focus

C Exposure characterization

C.1 Workplace factors associated with harmful skin exposures

C.2 Description of factors influencing exposure conditions

C.2.A Exposure intensity/frequency/duration

C.2.B Exposure concentration

C.2.C Skin area affected

C.2.E Uptake

D Hazard identification

D.1 Potential health effects resulting from specific chemicals

D.1.B Allergic contact dermatitis/sensitization

D.1.C Systemic toxicity

This comprehensive reference by over 40 clinician contributors discusses diagnosis, treatment, and prevention of occupational skin disease. This chapter addresses uptake, biotransformation, exposure/reaction patterns, and effects of specific chemicals.

29

Article ID:

Ansell Chemsafe [2005]. Ansell Chemsafe.

[www.ansellchemsafe.com/Default.aspx].

Resource type:

Web site

Educational materials:

No

Number of references:

Industries/occupations:

General—overview

Specific process:

Chemical:

General—overview

Specific chemicals: 
Mixtures:

Audience:

Topics addressed:

Summary:
No

General

A Overview

A.1 Occurrence of skin exposures in the workplace

E Risk management

E.1 Overview of skin exposure control options

E.3 "Best practices"/guidelines/recommendations

E.3.D PPE and PPE rules

Ansell is an Australian chemical glove manufacturer. Their Web site includes general information on dermal exposure to chemicals and protecting the skin as well as a software program, SpecwareTM, that can be used to assist in the glove selection process. Specware provides the user with glove use recommendations for a variety of commonly used chemicals. The information can be accessed through the software program on their Web site or with their hardcopy Specware guide which is available on request.
Article ID:

Citation:

Resource type:

Educational materials:

Number of references:

Industries/occupations:

Specific process:

Chemical:

Specific chemicals:

Mixtures:

Audience:

Topics addressed:

\section{0}

Antezana M, Parker F [2003]. Occupational contact

dermatitis. Immunol Allergy Clin North Am 23(2):269-90.

Journal article—review, meta-analysis

No

43

Agricultural, Beauty/Cosmetology, Construction, Forestry/ Fisheries, Manufacturing-Other, Service-Medical

Painting

Printing

Forestry

Electronics

heavy metals/inorganic compounds, organic dyes, pesticides, rubber additives, other: adhesives, paints

paraphenylenediamine, nickel, chromium, ethylenediamine, thimerosal

No

Professional

A Overview

A.2 Health hazards resulting from skin exposure to chemicals

B Surveillance and clinical aspects 
Summary:

Article ID:

\section{Citation:}

Resource type:

Educational materials:

Number of references:

Industries/occupations:

Specific process:

Chemical:

Specific chemicals:

Mixtures:

Audience:

Topics addressed:
B.4 Clinical protocols for recognition of skin exposure health effects

C Exposure characterization

C.4 Direct methods to measure exposure

C.4.B Skin

D Hazard identification

D.1 Potential health effects resulting from specific chemicals

D.1.A Irritant contact dermatitis

D.1.B Allergic contact dermatitis/sensitization

F Risk management

F.1 Exposure control strategies

F.1.D PPE and PPE rules

F.1.E Skin management, barrier creams, moisturizers, cleansers, and rubs

This paper presents the epidemiology, pathophysiology, and symptomology of occupational dermatitis, as well as diagnostic tests for dermatitis. There is some discussion of at-risk occupations, common allergens and irritants, and preventive management.

\section{1}

Askin DP, Volkmann M [1997]. Effect of personal hygiene on blood lead levels of workers at a lead processing facility. AIHA J 58(10):752-53.

Journal article_-primary

No

3

Waste management

Heavy metals/inorganic compounds

lead

No

Professional

C Exposure characterization

C.1 Workplace factors associated with harmful skin exposures

C.4 Direct methods to measure exposure

C.4.B Skin 
Summary:

F Risk management

F.1 Exposure control strategies

F.1.C Work practice/Administrative controls

At a lead processing plant, lead was measured for 24 workers who were confident that their hands were clean. Samples were obtained by cleaning one hand with a wipe. Workers with more than one year's experience had a significantly positive correlation between lead on their hand and blood lead level, suggesting that lead on the skin enters the bloodstream. The route of entry was not investigated.

Article ID:

32

Citation:

ASTM International [2006]. [www.astm.org/].

Resource type:

Web site

Educational materials:

No

Number of references:

Industries/occupations:

General-overview

Specific process:

Chemical:

Coolants, plastics and resins

Specific chemicals:

Isocyanates, metalworking fluids

Mixtures:

No

Audience:

Professional

Topics addressed:

C Exposure characterization

C.1 Workplace factors associated with harmful skin exposures

C.4 Direct methods to measure exposure

C.4.B Skin

D Hazard identification

D.1 Potential health effects resulting from specific chemicals

D.1.B Allergic contact dermatitis/sensitization

F Risk management

F.1 Exposure control strategies

F.1.A Substitution

F.1.B Engineering controls

F.1.C Work practice/Administrative controls

F.1.D PPE and PPE rules 
Summary:

Article ID:

33
ASTM International is a voluntary standards development organization. Standards developed at ASTM are the work of ASTM members. These technical experts represent producers, users, consumers, government, and academia from over 100 countries. Standards and guides are available for sale on their Web site.

Some of the ASTM standards and guides relevant to dermal exposure include:

- E1497-05 Standard Practice for Safe Use of WaterMiscible Metal Removal Fluids-This practice provides guidelines for the selection and safe use of metal removal fluids, additives, and antimicrobials. This includes product selection, storage, dispensing, and maintenance.

- Standard E 1302, Guide for Acute Animal Toxicity Testing of Water-Miscible Metalworking Fluids-This guide defines acute animal toxicity tests and presents references for procedures that assess the acute toxicity of water-miscible metalworking fluid concentrates as manufactured.

- WK8210 Standard Guide to Test Methods for Personal Protective Equipment Intended for Homeland Security Applications - This guide provides a listing of test methods for personal protective equipment (PPE) intended to protect first and second responders, casualty receivers, and remediation personnel involved in homeland security incidents.

- STP 1408 ISOCYANATES: Sampling, Analysis, and Health Effects - 11 peer-reviewed papers on topics such as, isocyanate determination in atmospheres; sampling strategy and control; and personal protective equipment.

- F1296-03 Standard Guide for Evaluating Chemical Protective Clothing - This guide is intended to promote the proper selection, use, maintenance, and understanding of the limitations of chemical protective clothing by users, employers, employees, and other persons involved in programs requiring CPC. 
Citation:

Resource type:

Educational materials:

Number of references:

Industries/occupations:

Specific process:

Chemical:

Specific chemicals:

Mixtures:

Audience:

Topics addressed:

Summary:
Balsat A, De Graeve J, Mairiaux P [2003]. A structured strategy for assessing chemical risks, suitable for small and medium-sized enterprises. Ann Occup Hyg 47(7): 549-56.

Journal article-primary

No

26

No

Professional

E Risk assessment

E.1 Guidelines for risk assessment or analysis

E.1.B Systemic health effects

E.2 Example of risk assessments

The authors present Regetox, a two-step approach for assessing chemical health risks. The first step uses the method developed in France by the l'Institut National de Recherche et de Sécurité (INRS) to rank potential risk. The second step uses the COSHH method and EASE model established by the UK Health \& Safety Executive to assess chemical risk using occupational exposure limits. The authors call Regetox a useful tool for chemical risk assessment in small- and medium-sized enterprises (MSEs).
Article ID:

Citation:

Resource type:

Educational materials:

Number of references:

Industries/occupations:

Specific process:

Chemical:

Specific chemicals:

Mixtures:

Audience:

\section{4}

Basketter DA, Flyvholm MA, Menne T [1999].

Classification criteria for skin-sensitizing chemicals: A commentary. Contact Dermatitis 40(4):175-82.

Journal article_-review, meta-analysis

No

35

No

Professional 
Topics addressed:

Summary:

Article ID:

Citation:

Resource type:

Educational materials:

Number of references:

Industries/occupations:

Specific process:

Chemical:

Specific chemicals:

Mixtures:

Audience:

Topics addressed:
A Overview

A.3 Investigation, intervention, and control of occupational skin exposures

A.5 Dermal regulations and skin notations

D Hazard identification

D.1 Potential health effects resulting from specific chemicals

D.1.A Irritant contact dermatitis

D.1.B Allergic contact dermatitis/sensitization

D.1.C Systemic toxicity

This article reviews the benefits and limitations of systems to classify substances with high dermal potency (either significant skin sensitizers or important contact allergens). Included are discussions of strategies used by the European Union, the World Health Organization, and the United States. Such information is necessary for proper risk assessment and management of skin sensitizers.

35

Basketter DA, Evans P, Gerberick GF, Kimber IA [2002]. Factors affecting thresholds in allergic contact dermatitis: Safety and regulatory considerations. Contact Dermatitis 47(1):1-6.

Journal article—review, meta-analysis

No

39

\section{No}

Professional

A Overview

A.4 Skin physiology and function as barriers to chemical insults

D Hazard identification

D.2 Summaries of health effects, dose-response relationships

D.4 Other 
Summary:

Article ID:

Citation:

Resource type:

Educational materials:

Number of references:

Industries/occupations:

Specific process:

Chemical:

Specific chemicals:

Mixtures:

Audience:

Topics addressed:

Summary:
This article examines the nature of thresholds in allergic contact dermatitis. These thresholds vary according to whether skin exposure is transient or prolonged, open or occluded, and single or repeated, as well as the condition of the skin, the presence of inflammation, and the vehicle in which a chemical sensitizer comes into contact with the skin. Recommendations are provided for safety evaluation and dermal regulations. Allergic potencies also are provided for 38 chemicals using both guinea pig and lymph node assay data.

\section{6}

Baynes RE [2005]. Dermal absorption of cutting fluid mixtures. Raleigh, NC: Center for Chemical Toxicology Research and Pharmacokinetics, College of Veterinary Medicine, North Carolina State University.

Technical publication/report

No

38

Manufacturing-Machining industry

Coolants, heavy metals/inorganic compounds, petroleum products \& lubricants, solvents, cutting fluid additives

Linear alkibenzene sulfonate (LAS), sulfate ricinolei acid (RA), tiazine, nickel, trichloroethylene (TCE), triethanolamine $\mathrm{N}$-nitrosodiethanolamine

Yes

Professional

C Exposure characterization

C.2 Description of factors influencing exposure conditions

C.2.E Uptake

D Hazard identification

D.1 Potential health effects resulting from specific chemicals

D.1.A Irritant contact dermatitis

D.3 Characterization protocols

D.3.E Measurement of skin permeation rates and reservoir effects

This document reports results from the testing of several cutting fluid additives and contaminants to ascertain the influence of chemical mixtures on dermal disposition and 
cutaneous toxicity. The research examined three specific additives: linear alkibenzene sulfonate (LAS), sulfate ricinolei acid (RA), and tiazine with regard to dermal absorption, physiochemical interactions, and the effect on solvent (TCE) permeability.

Article ID:

37

Citation:

Resource type:

Educational materials:

Number of references:

Industries/occupations:

Specific process:

Chemical:

Specific chemicals:

Mixtures:

Audience:

Topics addressed:
Bello D, Sparer J, Redlich CA, Ibrahim K, Stowe MH, Liu Y [2007]. Slow curing of aliphatic polyisocyanate paints in automotive refinishing: A potential source for skin exposure." J Occup Environ Hyg 4(6):406-11.

Journal article—primary

No

26

Manufacturing, Automotive refinishing painting

Autobody workers

Paints

Aliphatic isocyanates, hexamethylene diisocyanate (pHDI) isophorone diisocyanate (pIPDI), HDI, MDI, TDI

No

Professional

A Overview

A.1 Occurrence of skin exposures in workplace

C Exposure characterization

C.2 Description of factors influencing exposure conditions

C.2.D Other

C.4 Direct methods to measure exposure

C.4.A Surfaces

The paint of recently painted automobiles contains unbound isocyanate species which pose less risk after drying. This study investigated how long such paint takes to cure. From study results, the authors conclude that unbound isocyanates remain present up to 120 hours for typical paint formulations and for one month for others, presenting a risk to autobody workers.
Article ID:

Citation:

\section{8}

Bello D, Herrick CA, Smith TJ, Woskie SR, Streicher RP,

Cullen MR, Liu Y, Redlich CA [2007]. Skin exposure to 
Resource type:

Educational materials:

Number of references:

Industries/occupations:

Specific process:

Chemical:

Specific chemicals:

Mixtures:

Audience:

Topics addressed:

Summary: isocyanates: Reasons for concern. Environ Health Perspect 115(3):328-35.

Journal article—review, meta-analysis

No

93

Manufacturing-Chemical

Polyurethane production

Plastics and resins

Isocyanates, methylene diisocyanate (MDI), toluene diisocyanate (TDI) polymeric hexamethylene diisocyanate (pHDI), isophorone diisocyanate (pIPDI), glues, foam insulation

Yes

Professional

A Overview

A.1 Occurrence of skin exposures in workplace

B Surveillance and clinical aspects

B.1 Surveillance study reporting incidences of occupational skin exposures

B.1.B Skin exposure minor focus

C Exposure characterization

C.1 Workplace factors associated with harmful skin exposures

C.2 Description of factors influencing exposure conditions

C.2.E Uptake

C.4 Direct methods to measure exposure

C.4.B Skin

D Hazard identification

D.1 Potential health effects resulting from specific chemicals

D.1.B Allergic contact dermatitis/sensitization

D.1.D Other health effects

D.4 Other

F Risk management

F.1 Exposure control strategies

F.1.D PPE and PPE rules

This resource discusses the results of a literature review of 800 animal and human studies on isocyanate skinexposure methods, workplace skin exposure, skin 
absorption, and the role of skin exposure in isocyanate sensitization and asthma.

Article ID:

Citation:

Resource type:

Educational materials:

Number of references:

Industries/occupations:

Specific process:

\section{Chemical:}

Specific chemicals:

Mixtures:

Audience:

Topics addressed:
39

Benford DJ, Cocker J, Sartorelli P, Schneider T, van Hemmen J, Firth JG [1999]. Dermal route in systemic exposure. Scand J Work Environ Health 25(6):511-20.

Journal article-review, meta-analysis

No

31
No

Professional

C Exposure characterization

C.2 Description of factors influencing exposure conditions

C.2.E Uptake

C.4 Direct methods to measure exposure

C.4.A Surfaces

C.4.B Skin

C.4.C Biomonitoring

C.5 Exposure modeling

D Hazard identification

D.3 Characterization protocols

D.3.E Measurement of skin permeation rates and reservoir effects

D.3.F QSARs—-development, validation, and application

This article discusses methods for measuring skin and surface contamination, biological monitoring, and estimating dermal uptake via in vitro and in vivo methods. The article also discusses how standardized components of exposure characterization can be developed, and how they can be used to support a generic approach to dermal risk assessment and allow for the development of workplaceappropriate assessment strategies. 
Article ID:

Citation:

Resource type:

Educational materials:

Number of references:

Industries/occupations:

Specific process:

Chemical:

Specific chemicals:

Mixtures:

Audience:

Topics addressed:

Summary:
40

Boeniger M [2002]. Chemical protective clothing and the skin: Practical considerations. In: Anna DH, ed. Chemical Protective Clothing Series. Fairfax, VA: American Industrial Hygiene Association. 549-PC-02:1-48.

Book/monograph, chapter

No

170
No

Professional

A Overview

A.4 Skin physiology and function as barriers to chemical insults

B Surveillance and clinical aspects

B.1 Surveillance study reporting incidences of occupational skin exposures

B.1.A Skin exposure major focus

D Hazard identification

D.1 Potential health effects resulting from specific chemicals

D.1.A Irritant contact dermatitis

F Risk management

F.1 Exposure control strategies

F.1.D PPE and PPE rules

This chapter describes environmental and nonenvironmental factors affecting dermal absorption and how PPE reduces absorption. Factors affecting dermal absorption that are covered include: anatomical differences, interindividual differences, physical damage to skin and temperature, humidity, the chemical vehicle, and skin occlusion.
Article ID:

Citation:

\section{1}

Boeniger MF [2000]. Exposure and absorption of hazardous materials through the skin. Int J Occup Environ Health 6(2):148-50. 
Resource type:

Educational materials:

Number of references:

Industries/occupations:

Specific process:

Chemical:

Specific chemicals:

Mixtures:

Audience:

Topics addressed:

Summary:
Other-commentary

No

19
No

Professional

A Overview

A.5 Dermal regulations and skin notations

C Exposure characterization

C.2 Description of factors influencing exposure conditions

\section{C.2.E Uptake}

This letter to the editor from NIOSH summarizes the history of attempts to quantify dermal permeation rates. Rates, based upon LD50 or permeation coefficients from saturated aqueous solutions, form the bases for OSHA skin notations, ACGIH threshold limit values (TLVs), OSHA permissible exposure limits (PELs), and NIOSH recommended exposure limits (RELs). As a result of differing laboratory methodologies, these limits may vary by several orders of magnitude. The letter offers recommendations for those offering additional skin exposure guidance and permeation criteria.

Article ID:

42

Citation:

Resource type:

Educational materials:

Number of references:

Industries/occupations:

Specific process:

Chemical:

Specific chemicals:

Mixtures:

Audience:

Topics addressed:
Boeniger MF, Klingner TD [2002]. In-use testing and interpretation of chemical-resistant glove performance. Appl Occup Environ Hyg 17(5):368-78.

Journal article—review, meta-analysis

No

49

No

Professional

B Surveillance and clinical aspects 
Summary:

$\begin{array}{ll}\text { B.2 } & \text { Loss of workdays and impact on productivity } \\ \text { C } & \text { Exposure characterization } \\ \text { C.4 } & \text { Direct methods to measure exposure } \\ \text { C.4.B } & \text { Skin } \\ \text { C.5 } & \text { Exposure modeling } \\ \text { F } & \text { Risk management } \\ \text { F.1 } & \text { Exposure control strategies } \\ \text { F.1.D } & \text { PPE and PPE rules }\end{array}$

This article reviews methods for testing glove performance during actual use and offers a method for estimating acceptable exposure guidance criteria for evaluation of chemicals that are systemically absorbed.

Article ID:

43

Citation:

Resource type:

Educational materials:

Number of references:

Industries/occupations:

Specific process:

Chemical:

Specific chemicals:

Mixtures:

Audience:

Topics addressed:

Summary:
Boeniger MF, Ahlers HW [2003]. Federal government regulation of occupational skin exposure in the USA. Int Arch Occup Environ Health 76(5):387-99.

Journal article-review, meta-analysis

No

40
No

General

A Overview

A.3 Dermal regulations and skin notations

This paper provides an overview of federal regulations of dermal exposure. An analysis of 14 federal regulations and three agencies that regulate occupational skin exposure in the United States is presented. USEPA requires reporting of chemical health effects information which it uses to assess exposure risk. The Food and Drug Administration (FDA) regulates the labeling of cosmetics and requires safety data on new health products. OSHA regulates workplace safety and assesses compliance through field inspections. This paper evaluates how well the regulations prevent exposure and recommends measures to further protect workers from occupational skin hazards. 
Article ID:

Citation:

Resource type:

Educational materials:

Number of references:

Industries/occupations:

Specific process:

Specific chemicals:

Mixtures:

Audience:

Topics addressed:

Summary:
Chemical:

\section{4}

Boman A, Maibach HI [2000]. Percutaneous absorption of organic solvents. Int J Occup Environ Health 6(2):93-95.

Journal article—review, meta-analysis

No

37

Solvents

n-Butanol, toluene, 1,1,1-trichloroethane

No

Professional

C Exposure characterization

C.2 Description of factors influencing exposure conditions

C.2.E Uptake

C.5 Exposure modeling

D Hazard identification

D.2 Summaries of health effects, dose-response relationships

This paper discusses factors affecting percutaneous absorption of organic solvents including the pathway, toxicity, and environmental factors. Absorption rates vary considerably. Amphiphilic solvents have higher absorption rates. Nonoccluded repeated exposure results in less absorption than continuous contact. Ventilation reduces absorption.

\section{5}

Article ID:

Boman A [2005]. Protective gloves for occupational use. Boca Raton, FL: CRC Press.

Resource type:

Book/monograph, whole

Educational materials:

No

Number of references:

1014

Industries/occupations:

Service-Medical

Specific process:

Chemical:

Latex, pesticides

Specific chemicals:

Mixtures:

No 
Audience:

Topics addressed:

Summary:

Article ID:

Citation:

Resource type:

Educational materials:

Number of references:

Industries/occupations:

Specific process:

Chemical:

Specific chemicals:

Mixtures:

Audience:

Topics addressed:
Professional

$\begin{array}{ll}\text { A } & \text { Overview } \\ \text { A.5 } & \text { Dermal regulations and skin notations } \\ \text { F } & \text { Risk management } \\ \text { F.1 } & \text { Exposure control strategies } \\ \text { F.1.D } & \text { PPE and PPE rules }\end{array}$

This book covers quality standards and requirements related to the side effects of glove use, advanced technical standard test method results, permeation and penetration test data, medical reports on side effects, and applications in the glove selection process. It discusses protective glove use safety and use directives, regulations, and requirements in Europe and the United States, standard quality control test methods, in vivo testing with animals, and clinical diagnostic testing in patients.

\section{6}

Bos PM, Brouwer DH, Stevenson H, Boogaard PJ, de Kort WL, van Hemmen JJ [1998]. Proposal for the assessment of quantitative dermal exposure limits in occupational environments, part 1. Development of a concept to derive a quantitative dermal occupational exposure limit. Occup Environ Med 55(12):795-804.

Journal article—review, meta-analysis

No

48

cyclophosphamide, 4,4-methylene dianiline (MDA)

No

Professional

A Overview

A.5 Dermal regulations and skin notations

C Exposure characterization

C.2 Description of factors influencing exposure conditions

C.2.A Exposure intensity/frequency/duration

C.2.B Exposure concentration

C.2.C Skin area affected 
Summary:
C.2.D Other
C.2.E Uptake
D Hazard identification
D.1 Potential health effects resulting from specific chemicals
D.1.A Irritant contact dermatitis
D.1.B Allergic contact dermatitis/sensitization
D.3 Characterization protocols
D.3.E Measurement of skin permeation rates and reservoir effects

The authors argue that quantitative dermal occupational exposure limits (DOEL) should be developed, similar to respiratory occupational exposure limits (OELs), to replace today's qualitative "skin notation" warnings. The authors present a procedure for developing DOELs for the total dose deposited on the skin during a working shift and use their procedure to develop a DOEL for cyclophosphamide and 4,4-methylene dianiline (MDA). They conclude that the DOEL that they developed is relevant and useful, but further research is needed to show whether the procedure is applicable to other chemicals.

Article ID:

47

Citation:

Resource type:

Educational materials:

Number of references:

Industries/occupations:

Specific process:

Chemical:

Specific chemicals:

Mixtures:

Audience:

Topics addressed:
CDC [2002]. Guideline for hand hygiene in health-care settings. Recommendations of the Healthcare Infection Control Practices Advisory Committee and the HICPAC/ SHEA/APIC/IDSA Hand Hygiene Task Force. MMWR Recomm Rep 51(RR16):1-45.

Guideline

No

423

No

Professional

A Overview

A.4 Skin physiology and function as barriers to chemical insults

C Exposure characterization 
Summary:

$$
\begin{array}{ll}
\text { C.5 } & \text { Exposure modeling } \\
\text { F } & \text { Risk management } \\
\text { F.1 } & \text { Exposure control strategies } \\
\text { F.1.C } & \text { Work practice/Administrative controls } \\
\text { F.1.E } & \begin{array}{l}
\text { Skin management, barrier creams, moisturizers, } \\
\text { cleansers, and rubs }
\end{array}
\end{array}
$$

The Guideline for Hand Hygiene in Health-Care Settings provides healthcare workers with a review of data regarding handwashing, hand antisepsis, and barrier creams as well as recommendations to improve handhygiene practices and to reduce transmission of pathogenic microorganisms.

Article ID:

\section{Citation:}

Resource type:

Educational materials:

Number of references:

Industries/occupations:

Specific process:

Chemical:

Specific chemicals:

Mixtures:

Audience:

Topics addressed:

Summary:

\section{8}

Brondeau MT, Hesbert A, Beausoleil C, Schneider O

[1999]. To what extent are biomonitoring data available in chemical risk assessment? Hum Exp Toxicol 18(5):322-26.

Journal article_-review, meta-analysis

No

58

Solvents

Styrene; TCE; acrylonitrile; buta-1,3-diene;

cyclohexane; 1,4-dichlorobenzene; hydrogen fluoride;

2-(2-methoxyethoxy)ethanol; alkanes-C10-13-chloro; benzene-C10-13-alkyl derivatives; bis(pentabromophenyl) ether; diphenyl ether, octabromo derivative

No

Professional

C Exposure characterization

C.2 Description of factors influencing exposure conditions

C.2.E Uptake

C.4 Direct methods to measure exposure

C.4.C Biomonitoring

Biomonitoring information is helpful in assessing chemical exposure and would result in more accurate risk assessments. The availability of biomonitoring and metabolism animal data, skin penetration ability, and atmospheric threshold limits were examined for 12 substances: 
styrene; TCE; acrylonitrile; buta-1,3-diene; cyclohexane; 1,4-dichlorobenzene; hydrogen fluoride; 2-(2-methoxyethoxy) ethanol; alkanes-C10-13-chloro; benzene-C10-13-alkyl derivatives; bis(pentabromophenyl)ether; and diphenylether, octabromo derivative. The availability of biomonitoring data varied from widely available (for styrene and TCE) to lacking or scarce.

Article ID:

\section{Citation:}

Resource type:

Educational materials:

Number of references:

Industries/occupations:

Specific process:

Chemical:

Specific chemicals:

Mixtures:

Audience:

Topics addressed:

Summary:

\section{9}

Brouwer DH, Boeniger MF, van Hemmen J [2000]. Hand wash and manual skin wipes. Ann Occup Hyg 44(7):501-10.

Journal article-review, meta-analysis

No

39
No

Professional

C Exposure characterization

C.4 Direct methods to measure exposure

C.4.B Skin

This paper reviews both hand wash and skin wipe techniques of dermal exposure sampling for sampling efficiency. Sampling protocols hamper comparisons of study results. The authors conclude harmonization of sampling protocols will be a first step in creating a database for better understanding the influence of sampling parameters on the performance of removal techniques to assess dermal exposure.

Article ID:

50

Citation:

Resource type:

Educational materials:
Brouwer DH, Semple S, Marquart J, Cherrie JW [2001]. A dermal model for spray painters. Part I: Subjective exposure modelling of spray paint deposition. Ann Occup Hyg 45(1):15-23.

Journal article_-primary

No 
Number of references:

Industries/occupations:

Specific process:

Chemical:

Specific chemicals:

Mixtures:

Audience:

Topics addressed:

Summary:
21

Construction

Spray painting

No

Professional

C Exposure characterization

C.1 Workplace factors associated with harmful skin exposures

C.2 Description of factors influencing exposure conditions

C.2.A Exposure intensity/frequency/duration

C.2.B Exposure concentration

C.2.C Skin area affected

C.2.E Uptake

C.5 Exposure modeling

Part 1 of 2. This paper presents a model based upon "a process-based, structured approach" that estimates both occupational dermal exposure and uptake of solvents, using airless spray painters as an example. Estimates are based upon spray technique, object shape, workers' individual work practices, droplet formation, and deposition. Predicted exposure showed reasonable correlation with the actual measured exposure, and the authors conclude that a structured, process-based approach has the potential to produce reliable estimates of dermal exposure, and they call for additional field studies.

Part 1 presents this "structured, subjective assessment" of dermal exposure estimation and evaluates its reliability.
Article ID:

Citation:

Resource type:

Educational materials:

Number of references:

Industries/occupations:

\section{1}

Brouwer DH, Aitken RJ, Oppl R, Cherrie JW [2005].

Concepts of skin protection: Considerations for the evaluation and terminology of the performance of skin protective equipment. J Occup Environ Hyg 2(9):425-34.

Journal article—review, meta-analysis

No

24 
Specific process:

Chemical:

Specific chemicals:

Mixtures:

Audience:

Topics addressed:
No

Professional

A Overview

A.3 Investigation, intervention, and control of occupational skin exposures

A.4 Skin physiology and function as barriers to chemical insults

C Exposure characterization

C.2 Description of factors influencing exposure conditions

C.2.A Exposure intensity/frequency/duration

C.2.B Exposure concentration

C.2.C Skin area affected

C.2.E Uptake

C.5 Exposure modeling

F Risk management

F.1 Exposure control strategies

F.1.D PPE and PPE rules

This article proposes a common dermal exposure glossary, including processes involved in transport, loading, uptake, and personal protective equipment. It presents both exposure loading and skin protective equipment models and presents performance data for skin protective equipment.
Article ID:

\section{Citation:}

Resource type:

Educational materials:

Number of references:

Industries/occupations:

Specific process:

Chemical:

Specific chemicals:

Mixtures:

\section{2}

Brown JW III [2002]. Chemical hand protection. Occup Health Saf 71(2):56-68.

Magazine article

Yes

0

Solvents, ketones, acids, esters

Benzene

Yes 
Audience:

Topics addressed:

Summary:

Article ID:

Citation:

Resource type:

Educational materials:

Number of references:

Industries/occupations:

Specific process:

Chemical:

Specific chemicals:

Mixtures:

Audience:

Topics addressed:
General

E Risk management

E.3 "Best practices"/guidelines/recommendations

E.3.D PPE and PPE rules

E.3.E Skin management, barrier creams, moisturizers, cleansers, and rubs

This article summarizes the merits of various work gloves including natural rubber, nitrile, neoprene, hypalon, butyl, viton, and ethylene vinyl alcohol (EVOH). This article also discusses selection criteria including chemical resistance, finish and lining, and glove comfort and dexterity. This article also briefly discusses skin conditions and PPE training.

53

Brown T [2004]. Strategies for prevention: Occupational contact dermatitis. Occup Med (Lond) 54(7):450-57.

Journal article—review, meta-analysis

No

109

No

Professional

B Surveillance and clinical aspects

B.1 Surveillance study reporting incidences of occupational skin exposures

B.1.A Skin exposure major focus

B.2 Loss of workdays and impact on productivity

B.3 Surveillance study protocols/procedures for gathering data

F Risk management

F.1 Exposure control strategies

F.1.A Substitution

F.1.B Engineering controls

F.1.D PPE and PPE rules 
Summary:

Article ID:

Citation:

Resource type:

Educational materials:

Number of references:

Industries/occupations:

Specific process:

Chemical:

Specific chemicals:

Mixtures:

Audience:

Topics addressed:

Summary:
F.1.E Skin management, barrier creams, moisturizers, cleansers, and rubs

F.2 Protocols for risk management

F.2.B Development of approach to achieve exposure reduction goal

This paper presents strategies for preventing occupational contact dermatitis (OCD) including elimination/ substitution, engineered/technical controls, personal protective equipment (PPE), identifying susceptible individuals, education, training and surveillance.

\section{4}

Bureau of Labor Statistics (BLS) [2005]. BLS industry

illness and injury data. [www.bls.gov/iif/oshsum.htm].

Web page

No

Agricultural, Beauty/Cosmetology, Cleaning/Janitorial/ Maid, Construction, Forestry/Fisheries, ManufacturingChemical, Manufacturing, Medical Services, Mining, Service-Food, Service-Medical, ServiceTransportation/Communications/Utility, Other

No

Professional

B Surveillance and clinical aspects

B.1 Surveillance study reporting incidences of occupational skin exposures

B.1.A Skin exposure major focus

B.1.B Skin exposure minor focus

B.2 Loss of workdays and impact on productivity

B.3 Surveillance study protocols/procedures for gathering data

The BLS is an independent national statistical agency that collects, processes, analyzes, and disseminates essential statistical data to the American public, the U.S. Congress, other Federal agencies, state and local governments, business, and labor. The BLS also serves as a statistical resource to the Department of Labor. The Injuries, 
Illnesses, and Fatalities Program provides data on illnesses and injuries on the job and data on worker fatalities, summarized by year. The data are presented in different forms, including illness rates for different industries by type of illness. Skin diseases and skin disorders are one of the types of illnesses reported.

Article ID:

\section{Citation:}

Resource type:

Educational materials:

Number of references:

Industries/occupations:

Specific process:

Chemical:

Specific chemicals:

Mixtures:

Audience:

Topics addressed:

Summary:

\section{5}

Burnett CA, Lushniak BD, McCarthy W, Kaufman J [1998]. Occupational dermatitis causing days away from work in U.S. private industry, 1993. Am J Ind Med 34(6):568-73.

Journal article—primary

No

15

Agricultural, Cleaning/Janitorial/Maid, Construction, Manufacturing, Service-Food, Service-Medical, Transportation/Communications/UtilityGroundskeepers, Gardeners, Mechanics, Printing Press Operators, Repairmen

Provides risk data from many occupational groups.

General—overview, cleaning agents, petroleum products \& lubricants, plastics and resins, solvents

Calcium hydroxide

Provides risk data for several chemical classes

No

Professional

B Surveillance and clinical aspects

B.1 Surveillance study reporting incidences of occupational skin exposures

B.1.A Skin exposure major focus

B.2 Loss of workdays and impact on productivity

B.3 Surveillance study protocols/procedures for gathering data

The authors examined the 8,835 cases of dermal exposure in the 1993 Annual Survey of Occupational Injuries and Illnesses from the BLS. The article presents considerable surveillance data including rates of occupational dermatitis, identifies the service sector with the greatest number of cases, the sectors with the highest exposure rates, and the chemicals causing the greatest number of exposures. 
Article ID:

Citation:

Resource type:

Educational materials:

Number of references:

Industries/occupations:

Specific process:

Chemical:

Specific chemicals:

Mixtures:

Audience:

Topics addressed:

Summary:

\section{6}

Byrne MA [2000]. Suction methods for assessing contamination on surfaces. Ann Occup Hyg 44(7):523-28.

Journal article—review, meta-analysis

No

21

No

Professional

C Exposure characterization

C.4 Direct methods to measure exposure

C.4.A Surfaces

C.5 Exposure modeling

This paper reviews commonly employed sampling techniques for occupational surfaces reported in the literature, removal efficiency, and applicability to dermal exposure assessment.
Article ID:

Citation:

Resource type:

Educational materials:

Number of references:

Industries/occupations:

Specific process:

Chemical:

Specific chemicals:

Mixtures:

Audience:

Topics addressed:

\section{7}

California Department of Industrial Relations (CADIR), Division of Labor Statistics and Research [2003]. [www.dir.ca.gov/DLSR/Injuries/2003/Menu.htm]. Web site

No

general—overview
No

Professional

B Surveillance and clinical aspects

B.1 Surveillance study reporting incidences of occupational skin exposures

B.1.A Skin exposure major focus 
Summary:

Article ID:

Citation:

Resource type:

Educational materials:

Number of references:

Industries/occupations:

Specific process:

Chemical:

Specific chemicals:

Mixtures:

Audience:

Topics addressed:
B.1.B Skin exposure minor focus

B.2 Loss of workdays and impact on productivity

The California Division of Labor Statistics and Research collects, compiles, and presents statistics and research relating to the condition of labor in the state. Data presented on their site include incidence rates and numbers of nonfatal occupational illnesses by industry sector and category of illness and the numbers of nonfatal occupational illnesses by selected industries and category of illness.

\section{8}

Canadian Centre for Occupational Health and Safety (CCOHS) [2005]. [www.ccohs.ca/].

Web site

No

General-overview

Multiple occupations

General-overview, corrosives, heavy metals/inorganic compounds, latex, particulates, petroleum products \& lubricants, solvents

Multiple chemicals

No

General

A Overview

A.1 Occurrence of skin exposures in the workplace

A.2 Health hazards resulting from skin exposure to chemicals

B Exposure characterization

B.1 Job/tasks, industries/processes, or chemicals associated with skin exposures

B.2 Factors that influence exposure conditions

B.2.B Exposure controls

C Hazard identification

C.3 Protocols/checklists to identify skin hazards in the workplace

E Risk management

E.3 "Best practices"/guidelines/recommendations

E.3.B Engineering controls 
Summary:

Article ID:

\section{Citation:}

Resource type:

Educational materials:
E.3.C Work practice/administration controls

E.3.D PPE and PPE rules

E.3.E Skin management, barrier creams, moisturizers, cleansers, and rubs

The Canadian Centre for Occupational Health and Safety is a Canadian federal government agency that promotes workplace health and safety by providing resources and programs on different health and safety topics. Their Web site contains general information, articles, news releases, products, and services related to occupational safety and health. Dermal exposure-related information can be found throughout the site. Good sources include:

- OSH Answers-This searchable page contains information on different topics on occupational health and safety. Information can be searched based on hazards present; occupations and workplaces; and diseases, disorders, and injuries, to name a few. OSH Answers contains hazard and prevention-related information for a variety of chemicals and chemical classes that are known skin hazards, including allergic contact dermatitis.

- Dermatitis, Allergic Contact Web page-This Web page covers occupations at risk, recognition, treatment, and preventive measures associated with allergic contact dermatitis.

- Dermatitis, Irritant Contact Web page-This Web page covers occupations at risk, recognition, treatment, and preventive measures associated with irritant contact dermatitis.

- WHMIS (Workplace Hazardous Materials Information System) label contains health hazard information. These labels are required by law. They use classifications to group chemicals with similar properties or hazards. Class E-corrosive material is a classification for compounds that can cause burns to eyes, skin, or respiratory system.
The Center to Protect Workers' Rights (CPWR) [2006]. [www.cpwr.com/indexstart.html].

Web site

Yes 
Number of references:

Industries/occupations:

Specific process:

Chemical:

Specific chemicals:

Mixtures:

Audience:

Topics addressed:

Summary:
Construction

General—overview, corrosives, solvents

No

General

A Overview

A.1 Occurrence of skin exposures in the workplace

C Hazard identification

C.2 Tables/charts/lists of hazards for specific chemicals

E Risk management

E.1 Overview of skin exposure control options

E.3 "Best practices"/guidelines/recommendations

E.3.C Work practice/administration controls

E.3.D PPE and PPE rules

E.3.E Skin management, barrier creams, moisturizers, cleansers, and rubs

E.4 Guidelines/recommendations for postexposure skin decontamination

CPWR is a nonprofit organization created by the Building and Construction Trades Department of the AFL-CIO. They provide applied research, training, and service to the construction industry. CPWR developed and maintains eLCOSH (for more information see eLCOSH, ID 63), which provides online construction-related health and safety information in English, Spanish, and other languages. The CPWR Web site also contains updates on conferences, publications, and news events associated with construction health and safety. Dermal exposure-related resources available on this Web site include:

- Hazard Alerts: Skin Problems in Construction, Beryllium, Solvents, and Lead

- The Construction Chart Book, which contains a chapter titled "Nonfatal Skin Diseases and Disorders in Construction."

- Save Your Skin: Tips on Preventing Skin Problems (brochure)

- The Construction Solutions Database, currently under development, will organize hazards by tasks and present ways to control those hazards. 
Article ID: $\quad 60$

Citation:

Resource type:

Educational materials:

Number of references:

Industries/occupations:

Specific process:

Chemical:

Specific chemicals:

Mixtures:

Audience:

Topics addressed:
CPWR [2000]. A safety and health practitioner's guide to skin protection. [www.cdc.gov/elcosh/docs/d0400/ d000458/d000458.html].

Brochure, pamphlet

Yes

Construction

Bricklayer, Carpenter, Masons, Hod Carrier, Plasterer, Terrazzo Worker, Tile Setter

Corrosives, hand cleansers, heavy metals/inorganic compounds, plastics and resins

Portland cement, CrVI

No

Professional

A Overview

A.1 Occurrence of skin exposures in the workplace

A.2 Health hazards resulting from skin exposure to chemicals

A.4 Skin physiology and function as barriers to chemical insults

B Surveillance and clinical aspects

B.1 Surveillance study reporting incidences of occupational skin exposures

B.1.A Skin exposure major focus

B.2 Loss of workdays and impact on productivity

B.3 Surveillance study protocols/procedures for gathering data

B.4 Clinical protocols for recognition of skin exposure health effects

C Exposure characterization

C.1 Workplace factors associated with harmful skin exposures

C.2 Description of factors influencing exposure conditions

C.2.A Exposure intensity/frequency/duration

C.2.B Exposure concentration

C.2.E Uptake

C.3 Checklists/questionnaires to quantify skin exposure incidences 


$\begin{array}{ll}\text { C.4 } & \text { Direct methods to measure exposure } \\ \text { C.4.A } & \text { Surfaces } \\ \text { D } & \text { Hazard identification } \\ \text { D.1 } & \begin{array}{l}\text { Potential health effects resulting from specific } \\ \text { chemicals }\end{array} \\ \text { D.1.A } & \text { Irritant contact dermatitis } \\ \text { D.1.B } & \text { Allergic contact dermatitis/sensitization } \\ \text { D.1.D } & \text { Other health effects } \\ \text { E } & \text { Risk assessment } \\ \text { E.1 } & \text { Guidelines for risk assessment or analysis } \\ \text { E.1.A } & \text { Localized health effects } \\ \text { F } & \text { Risk management } \\ \text { F.1 } & \text { Exposure control strategies } \\ \text { F.1.A } & \text { Substitution } \\ \text { F.1.B } & \text { Engineering controls } \\ \text { F.1.C } & \text { Work practice/Administrative controls } \\ \text { F.1.D } & \text { PPE and PPE rules } \\ \text { F.1.E } & \text { Skin management, barrier creams, moisturizers, } \\ & \text { cleansers, and rubs } \\ \text { F.2 } & \text { Protocols for risk management } \\ \text { F.2.C } & \text { Evaluation to demonstrate program/intervention } \\ & \text { effectiveness }\end{array}$

Summary:

This comprehensive guide was developed by the CPWR Consortium on Preventing Contact Dermatitis. Developed for safety and health practitioners, it covers dermal hazards associated with Portland cement work. It provides a detailed description of how to recognize, evaluate, and control dermal hazards. In addition to covering Portland cement, it also provides a thorough description of skin physiology and presents a model of skin disease within the context of occupational exposures to caustic chemicals and sensitizing agents. At the end, it provides a list of recommended resources, a best practices checklist, a skin symptoms questionnaire for workers, and a worker safety pamphlet. This brochure is available for download on the eLCOSH Web site ( see Article ID 63).

This guide contains the following chapters:
Ch. 1 Recognizing Skin Problems
Ch. 2 A New Model of Skin Disease
Ch. 3 Worksite Exposures 

Ch. 4 The Role of $\mathrm{pH}$
Ch. 5 Product Modification
Ch. 6 Best Protective Practices
Ch. 7 Resources
Ch. 8 Evaluating Your Success

Article ID:

Citation:

Resource type:

Educational materials:

Number of references:

Industries/occupations:

Specific process:

Chemical:

Specific chemicals:

Mixtures:

Audience:

Topics addressed:

\section{1}

CPWR [2001]. Physician's alert for occupational contact dermatitis among construction workers. [www.cdc.gov/ elcosh/docs/d0200/d000281/d000281.pdf].

Brochure, pamphlet

Yes

General-overview, Construction

Corrosives, hand cleansers

Portland cement, CrVI, lanolin

No

Professional

A Overview

A.1 Occurrence of skin exposures in workplace

A.2 Health hazards resulting from skin exposure to chemicals

B Surveillance and clinical aspects

B.4 Clinical protocols for recognition of skin exposure health effects

F Risk management

F.1 Exposure control strategies

F.1.E Skin management, barrier creams, moisturizers, cleansers, and rubs

Summary:

Developed by the CPWR, this physician's alert pamphlet was designed as information construction workers could bring with them on visits to a physician's office for skinrelated disorders. The pamphlet contains a table of skin disorders, possible work-related causes, diagnostic aids, and intervention and treatment options.

This pamphlet can be found online at the eLCOSH Web site (http://www.cdc.gov/elcosh/index.html). 
Article ID: $\quad 62$

Citation:

Resource type:

Educational materials:

Number of references:

Industries/occupations:

Specific process:

Chemical:

Specific chemicals:

Mixtures:

Audience:

Topics addressed:

Summary:
CPWR [2005]. An employer's guide to skin protection. [www.cdc.gov/elcosh/docs/d0400/d000457/d000457.html].

Brochure, pamphlet

Yes

25

Construction

Bricklayers, Carpenters, Masons, Hod Carrier, Plasterer, Terrazzo Worker, Tile Setters

Corrosives

Portland cement, CrVI

No

General

A Overview

A.1 Occurrence of skin exposures in the workplace

A.2 Health hazards resulting from skin exposure to chemicals

B Exposure characterization

B.1 Job/tasks, industries/processes, or chemicals associated with skin exposures

B.2 Factors that influence exposure conditions

B.2.A Exposure intensity/frequency

B.2.B Exposure controls

E Risk management

E.1 Overview of skin exposure control options

E.3 "Best practices"/guidelines/recommendations

E.3.C Work practice/administration controls

E.3.D PPE and PPE rules

E.4 Guidelines/recommendations for postexposure skin decontamination

This handbook for employers in the cement product industry (concrete, mortar, plaster, grout, stucco, and terrazzo) covers issues associated with dermal exposure identification, evaluation, control, and prevention. It offers recommendations to prevent employee skin problems.
Article ID:

\section{Citation:}

\section{3}

CPWR [2005]. Electronic library of construction occupational safety and health (eLCOSH). [www.cdc.gov/ elcosh/]. 
Resource type:

Educational materials:

Number of references:

Industries/occupations:

Specific process:

Chemical:

Specific chemicals:

Mixtures:

Audience:

Topics addressed:
Summary:
Web site

Yes

Construction

Corrosives, hand cleansers, heavy metals/inorganic compounds, soaps and detergents

Portland cement, CrVI

Yes

General

A Overview

A.1 Occurrence of skin exposures in the workplace

A.2 Health hazards resulting from skin exposure to chemicals

B Exposure characterization

B.1 Job/tasks, industries/processes, or chemicals associated with skin exposures

B.2 Factors that influence exposure conditions

B.2.B Exposure controls

B.3 Protocols/checklists to characterize exposure to skin hazards

C Hazard identification

C.3 Protocols/checklists to identify skin hazards in the workplace

D Risk assessment

D.1 Protocols/checklists to identify exposure risk

E Risk management

E.3 "Best practices"/guidelines/recommendations

E.3.B Engineering controls

E.3.C Work practice/administration controls

E.3.D PPE and PPE rules

E.3.E Skin management, barrier creams, moisturizers, cleansers, and rubs

E.4 Guidelines/recommendations for postexposure skin decontamination

The eLCOSH is intended to provide accurate, user-friendly information about safety and health for construction workers from different sources. The eLCOSH was developed by the CPWR through funding from NIOSH and is maintained by CPWR. Information on the Web 
site can be located by hazard, trade, job site, and other categories. Downloadable resources on this site related to dermal exposure to chemicals include the following:

- Save your Skin: Tips on Preventing Skin Problems-a general information brochure for workers.

- Chemical Glove Selection-a document produced by the University of Delaware Cooperative Extension on glove selection in agricultural settings.

- Physician's Alert: Skin Conditions-a brochure produced by the CPWR for workers to bring to their physicians office.

- An Employer's Guide To Skin Protection-a comprehensive document for employers covering a variety of issues associated with the evaluation, control, and prevention of dermal exposure to cement products, CrVI, and worksite cleansers.

- A Safety \& Health Practitioner's Guide to Skin Protection - a comprehensive document on dermal exposure developed for the person responsible for protecting the safety and health of workers using Portland cement products. Similar to the document produced for employers, this document goes into more depth and includes a worker safety pamphlet, a best practices checklist, and a symptoms questionnaire.

Article ID: 64

Citation:

Resource type:

Educational materials:

Number of references:

Industries/occupations:

Specific process:

Chemical: Specific chemicals:

Mixtures:

Audience:

Topics addressed:

\section{CDC [2006]. NASD: National Ag Safety Database.} [www.cdc.gov/nasd/].

Web site

Yes

Agricultural

Hand cleansers, pesticides

No

General

E Risk management

E.1 Overview of skin exposure control options

E.3 "Best practices"/guidelines/recommendations 


\author{
E.3.B Engineering controls \\ E.3.C Work practice/administration controls \\ E.3.D PPE and PPE rules \\ E.3.E Skin management, barrier creams, moisturizers, \\ cleansers, and rubs \\ E.4 Guidelines/recommendations for postexposure \\ skin decontamination \\ The National Ag Safety Database (NASD), developed \\ through funding from $\mathrm{NIOSH}$, is designed to provide a \\ national information resource on agricultural safety and \\ health issues, with the purpose of reducing agricultural \\ work-related illnesses and injuries. It contains a variety of \\ agricultural technical bulletins by topic, as well as posters \\ and videos for training. They also have Spanish language \\ material. The materials on this Web site are produced \\ by other sources, mostly state and university agriculture \\ extension offices. Dermal exposure bulletins include: Skin \\ Irritants, Pesticide-Contaminated Clothing Laundering, \\ and general information in Pesticide Exposure. Under the \\ topic "mixing, loading, and application" of chemicals/ \\ pesticides, there are a number of bulletins on the selection, \\ proper use, cleaning, and handling of PPE. Also available \\ are bulletins on other control measures and training \\ videotapes on pesticide safety.
}

\title{
Summary:
}

Article ID:

\section{5}

Citation:

Resource type:

Educational materials:

Number of references:

Industries/occupations:

Specific process:

Chemical:

Specific chemicals:

Mixtures:

Audience:

Topics addressed:
Chang H [2005]. Total body burden arising from a week's repeated dermal exposure to $\mathrm{N}, \mathrm{N}$-dimethylformamide (DMF). Occup Environ Med 62(3):151-56.

Journal article—primary

No

39

Solvents

$\mathrm{N}, \mathrm{N}$-dimethylformamide (DMF)

No

Professional

C Exposure characterization

C.2 Description of factors influencing exposure conditions

C.2.A Exposure intensity/frequency/duration 
Summary:

\author{
C.2.B Exposure concentration \\ C.2.C Skin area affected \\ C.2.E Uptake \\ C.4 Direct methods to measure exposure \\ C.4.B Skin \\ C.4.C Biomonitoring \\ D Hazard identification \\ D.2 Summaries of health effects, dose-response \\ relationships \\ This paper presents the results of a study designed to \\ estimate the contribution of skin absorption to total body \\ burden of N,N-dimethylformamide (DMF). The study \\ monitored 45 industrial workers and 20 nonDMF-exposed \\ subjects for DMF exposure via respiratory and dermal \\ routes. The control group showed no detectable exposure. \\ The study concluded that dermal exposure to DMF can \\ result in significant accumulation of DMF.
}

Article ID:

66

Citation:

Resource type:

Educational materials:

Number of references:

Industries/occupations:

Specific process:

Chemical:

Specific chemicals:

Mixtures:

Audience:

Topics addressed:
OPPTS [1996]. Applications International Corporation. Occupational dermal exposure assessment: a review of methodologies and field data: final report. Washington, DC: USEPA, Economics, Exposure and Technology Division, OPPTS.EPA 600-R-96-000 9-30-1996.

Technical publication/report

No

108

\section{No}

Professional

C Exposure characterization

C.4 Direct methods to measure exposure

C.4.A Surfaces

C.4.B Skin

C.5 Exposure modeling

D Hazard identification 
D.2 Summaries of health effects, dose-response relationships

Summary:

This paper summarizes a literature review on dermal exposure assessment and sampling methods. It includes a review of monitoring data on dermal exposure and identifies other methods used for predicting dermal exposure when monitoring data is not available. It evaluates the method for predicting dermal exposure developed by the Chemical Engineering Branch (CEB) of the USEPA OPPTS. The CEB method is evaluated under various scenarios and the authors revise or identify additional values and input parameters (e.g., quantity remained on skin, skin surface area). This review also makes recommendations to improve the CEB method.

Article ID:

67

Citation:

Resource type:

Educational materials:

Number of references:

Industries/occupations:

Specific process:

Chemical:

Specific chemicals:

Mixtures:

Audience:

Topics addressed:

Summary:
Cherrie JW, Brouwer DH, Roff M, Vermeulen R, Kromhout $\mathrm{H}$ [2000]. Use of qualitative and quantitative fluorescence techniques to assess dermal exposure. Ann Occup Hyg 44(7):519-22.

Journal article-review, meta-analysis

No

16

No

Professional

C Exposure characterization

C.4 Direct methods to measure exposure

\section{C.4.B Skin}

This paper reviews the literature on both quantitative and qualitative methods of dermal exposure using fluorescent tracers to estimate chemical uptake through the skin.
Article ID:

Citation:

\section{8}

Cherry N, Meyer JD, Adisesh A, Brooke R, Owen-Smith V, Swales C, Beck MH [2000]. Surveillance of occupational skin disease: EPIDERM and OPRA. Br J Dermatol 142(6). 
Resource type:

Educational materials:

Number of references:

Industries/occupations:

Specific process:

Chemical:

Specific chemicals:

Mixtures:

Audience:

Topics addressed:

Summary:
Journal article—primary

No

19

General—overview

Presents data from several large occupational groups, such as chemical operatives, metal assemblers, machine tool operatives, glass manufacturers, and printers.

General-overview

Presents data by several chemical agents, such as rubber, soaps, wet work, petroleum, and nickel.

No

Professional

A Overview

A.1 Occurrence of skin exposures in workplace

B Surveillance and clinical aspects

B.1 Surveillance study reporting incidences of occupational skin exposures

B.1.A Skin exposure major focus

This paper presents summary surveillance data, collected in the U.K., from the Occupational Physicians Reporting Activity (OPRA) and its predecessor, EPIDERM. OPRA is a voluntary surveillance mechanism that has collected occupational skin disease data from dermatologists and occupational physicians since 1993. Incidences by gender, age, occupational group, and chemical group are presented.

Article ID:

69

Citation:

Resource type:

Educational materials:

Number of references:

Industries/occupations:

Specific process:

Chemical:

Specific chemicals:
Chew AL, Maibach HI [2003]. Occupational issues of irritant contact dermatitis. Int Arch Occup Environ Health 76(5):339-46.

Journal article—review, meta-analysis

No

62

Agricultural, Beauty/Cosmetology, Cleaning/Janitorial/ Maid, Construction, Manufacturing, Service-Food, Service-Medical

Includes table of job categories and associated irritants

Cleaning agents, corrosives, pesticides, soaps and detergents, solvents 
Mixtures:

Audience:

Topics addressed:

Summary:
No

Professional

A Overview

A.2 Health hazards resulting from skin exposure to chemicals

B Surveillance and clinical aspects

B.1 Surveillance study reporting incidences of occupational skin exposures

B.1.A Skin exposure major focus

B.2 Loss of workdays and impact on productivity

B.4 Clinical protocols for recognition of skin exposure health effects

C Exposure characterization

C.4 Direct methods to measure exposure

C.4.B Skin

D Hazard identification

D.1 Potential health effects resulting from specific chemicals

D.1.A Irritant contact dermatitis

F Risk management

F.1 Exposure control strategies

F.1.D PPE and PPE rules

F.1.E Skin management, barrier creams, moisturizers, cleansers, and rubs

This paper reviews the various types of occupational irritant contact dermatitis along with epidemiological data, risk factors, pathophysiology, diagnosis, and management of irritant contact dermatitis.

Article ID:

70

Citation:

CLI [1999]. A guide to dermal exposure reduction. Des Plaines, IL: Colormetric Laboratories, Incorporated.

Resource type:

Other-Guideline from private lab

Educational materials:

No

Number of references:

0

Industries/occupations:

Specific process:

Chemical:

Specific chemicals: 


\begin{tabular}{|c|c|c|}
\hline Mixtures: & \multicolumn{2}{|l|}{ No } \\
\hline Audience: & \multicolumn{2}{|c|}{ Professional } \\
\hline \multirow[t]{5}{*}{ Topics addressed: } & $\mathrm{F}$ & Risk management \\
\hline & F.1 & Exposure control strategies \\
\hline & F.1.D & PPE and PPE rules \\
\hline & F.2 & Protocols for risk management \\
\hline & F.2.B & $\begin{array}{l}\text { Development of approach to achieve exposure } \\
\text { reduction goal }\end{array}$ \\
\hline Summary: & \multicolumn{2}{|c|}{$\begin{array}{l}\text { This pamphlet briefly outlines the benefit and contents } \\
\text { of a dermal exposure reduction program. This pamphlet } \\
\text { is available for download on their Web site (www.clilabs. } \\
\text { com). }\end{array}$} \\
\hline Article ID: & \multicolumn{2}{|l|}{71} \\
\hline Citation: & \multicolumn{2}{|c|}{$\begin{array}{l}\text { Colormetric Laboratories, Incorporated (CLI), } 2005 . \\
\text { [www.clilabs.com/]. }\end{array}$} \\
\hline Resource type: & \multicolumn{2}{|c|}{ Web site } \\
\hline Educational materials: & \multicolumn{2}{|l|}{ No } \\
\hline Number of references: & & \\
\hline Industries/occupations: & \multicolumn{2}{|c|}{ General-overview } \\
\hline Specific process: & & \\
\hline Chemical: & \multicolumn{2}{|c|}{ General-overview, plastics and resins } \\
\hline Specific chemicals: & \multicolumn{2}{|c|}{ isocyanates } \\
\hline Mixtures: & \multicolumn{2}{|l|}{ No } \\
\hline Audience: & \multicolumn{2}{|c|}{ Professional } \\
\hline \multirow[t]{13}{*}{ Topics addressed: } & $\mathrm{C}$ & Exposure characterization \\
\hline & C. 4 & Direct methods to measure exposure \\
\hline & C.4.A & Surfaces \\
\hline & C.4.B & Skin \\
\hline & C.4.C & Biomonitoring \\
\hline & $\mathrm{E}$ & Risk assessment \\
\hline & E.1 & Guidelines for risk assessment or analysis \\
\hline & E.1.A & Localized health effects \\
\hline & E.1.B & Systemic health effects \\
\hline & $\mathrm{F}$ & Risk management \\
\hline & F.1 & Exposure control strategies \\
\hline & F.1.A & Substitution \\
\hline & F.1.B & Engineering controls \\
\hline
\end{tabular}


F.1.C Work practice/Administrative controls

F.1.D PPE and PPE rules

F.1.E Skin management, barrier creams, moisturizers, cleansers, and rubs

Summary:

Colormetric Laboratories, Inc., provides biological monitoring analytical services and direct reading detection systems for evaluating surface and skin contamination. They produce the following detection systems:

- SWYPES ${ }^{\mathrm{TM}}$ detectors to determine where and when the skin exposures occurred that contributed to biological exposures.

- Permea-Tec ${ }^{\mathrm{TM}}$ Sensors which are breakthrough indicators for chemical protective gloves and can be used to determine glove life expectancy.

- D-TAM ${ }^{\mathrm{TM}}$ Safe Solvent for removing lipophilic compounds from skin.

- D-TAM ${ }^{\mathrm{TM}}$ Skin Cleansers, which are formulated to remove water-insoluble contaminants from the skin.

Article ID:

72

Citation:

Resource type:

Educational materials:

Number of references:

Industries/occupations:

Specific process:

Chemical:

Specific chemicals:

Mixtures:

Audience:

Topics addressed:

Summary:
Crassweller I [1999]. Helping hands: Skin care for the hands. Occup Hazards 61(8):58.

Magazine article

No

0

Cleaning agents

No

General

E Risk management

E.3 "Best practices"/guidelines/recommendations

E.3.E Skin management, barrier creams, moisturizers, cleansers, and rubs

E.4 Guidelines/recommendations for postexposure skin decontamination

Industrial workers exposed to harsh chemicals or who perform frequent hand-washing are susceptible to occupational skin diseases. This paper provides an 
overview of skin care and outlines the correct method of cleaning hands.

Article ID:

Citation:

Resource type:

Educational materials:

Number of references:

Industries/occupations:

Specific process:

Chemical:

Specific chemicals:

Mixtures:

Audience:

Topics addressed:

Summary:

\section{3}

Das R, Steege A, Baron S, Beckman J, Harrison R [2001]. Pesticide-related illness among migrant farm workers in the United States. Int J Occup Environ Health 7(4):303-12. Journal article—review, meta-analysis

No

79

Agricultural

Migrant farm workers

Pesticides

Organophosphates, carbamates, inorganic compounds, pyrethroids

No

Professional

A Overview

A.2 Health hazards resulting from skin exposure to chemicals

B Surveillance and clinical aspects

B.1 Surveillance study reporting incidences of occupational skin exposures

B.1.A Skin exposure major focus

F Risk management

F.1 Exposure control strategies

F.1.A Substitution

F.1.C Work practice/Administrative controls

This paper reviews a few pesticide categories (organophosphates, carbamates, inorganic compounds, and pyrethroids) that account for over half of all acute occupational illness cases among migrant farm workers in U.S. Most cases are caused by dermal exposures. Pesticide risk assessment should be based on acute toxicity, chronic toxicity, carcinogenic potency, volume applied, and magnitude of worker poisonings. Also discussed is the hierarchy of control measures, with a focus on substitution, establishing effective protections, enforcement, and education. This paper also contains a considerable amount of surveillance data. 


\begin{tabular}{|c|c|c|}
\hline Article ID: & \multicolumn{2}{|l|}{74} \\
\hline Citation: & \multicolumn{2}{|c|}{$\begin{array}{l}\text { Day GA, Stefaniak AB, Weston A, and Tinkle SS [2006]. } \\
\text { Beryllium exposure: dermal and immunological } \\
\text { considerations. Int Arch Occup Environ Health } \\
\text { 79(2):161-64. }\end{array}$} \\
\hline Resource type: & \multicolumn{2}{|c|}{ Journal article—review, meta-analysis } \\
\hline Educational materials: & \multicolumn{2}{|l|}{ No } \\
\hline Number of references: & \multicolumn{2}{|l|}{38} \\
\hline \multicolumn{3}{|l|}{ Industries/occupations: } \\
\hline \multicolumn{3}{|l|}{ Specific process: } \\
\hline \multicolumn{3}{|l|}{ Chemical: } \\
\hline Specific chemicals: & \multicolumn{2}{|c|}{ Beryllium } \\
\hline Mixtures: & \multicolumn{2}{|l|}{ No } \\
\hline Audience: & \multicolumn{2}{|c|}{ Professional } \\
\hline \multirow[t]{7}{*}{ Topics addressed: } & $\mathrm{B}$ & Surveillance and clinical aspects \\
\hline & B.1 & $\begin{array}{l}\text { Surveillance study reporting incidences of } \\
\text { occupational skin exposures }\end{array}$ \\
\hline & B.1.A & Skin exposure major focus \\
\hline & $\mathrm{D}$ & Hazard identification \\
\hline & D.1 & $\begin{array}{l}\text { Potential health effects resulting from specific } \\
\text { chemicals }\end{array}$ \\
\hline & D.1.B & Allergic contact dermatitis/sensitization \\
\hline & D.1.C & Systemic toxicity \\
\hline Summary: & \multicolumn{2}{|c|}{$\begin{array}{l}\text { This paper assesses the state of existing knowledge } \\
\text { concerning skin exposure to beryllium. It concludes that } \\
\text { the reduction in inhalation exposure to beryllium has } \\
\text { not reduced beryllium sensitization or chronic beryllium } \\
\text { diseases (CBD), suggesting that unchecked skin exposure is } \\
\text { causing continued prevalence. }\end{array}$} \\
\hline
\end{tabular}

Article ID: $\quad 75$

Citation:

Resource type:

Educational materials:

Number of references:

Industries/occupations:

Specific process:

Chemical:
Del Rosso J [2001]. Protecting the hand-skin barrier in the workplace. Occup Health Saf 70(9):116-20.

Magazine article

No

3 
Specific chemicals:

Mixtures: $\quad$ No

Audience: General

Topics addressed: A $\quad$ Overview

A.2 Health hazards resulting from skin exposure to chemicals

E Risk management

E.3 "Best practices"/guidelines/recommendations

E.3.D PPE and PPE rules

E.3.E Skin management, barrier creams, moisturizers, cleansers, and rubs

Summary:

This article recommends steps for proper skin care, including gloves and barrier creams.

Article ID:

76

Citation:

Resource type:

Educational materials:

Number of references:

Industries/occupations:

Specific process:

Chemical:

Specific chemicals:

Mixtures:

Audience:

Topics addressed:
Diepgen TL, Coenraads PJ [1995]. What can we learn from epidemiological studies on irritant contact dermatitis? Curr Probl Dermatol 23:18-27.

Journal article—review, meta-analysis

No

18

General—overview, Beauty/Cosmetology, Construction, Manufacturing-Other, Medical Services

Electroplaters, Metalworkers, Bricklayers
No

Professional

B Surveillance and clinical aspects

B.1 Surveillance study reporting incidences of occupational skin exposures

B.1.A Skin exposure major focus

B.3 Surveillance study protocols/procedures for gathering data

C Exposure characterization

C.1 Workplace factors associated with harmful skin exposures 
Summary:

Article ID:

Citation:

Resource type:

Educational materials:

Number of references:

Industries/occupations:

Specific process:

Chemical:

Specific chemicals:

Mixtures:

Audience:

Topics addressed:
This paper presents irritant contact dermatitis surveillance data and describes how age, gender, and race affect incidence and prevalence of irritant contact dermatitis.

77

Diepgen TL [1996]. Epidemiological studies on the prevention of occupational contact dermatitis. Curr Probl Dermatol 25:1-9.

Journal article-review, meta-analysis

No

13

Beauty/Cosmetology

Provides incidence among several occupational categories.

No

Professional

B Surveillance and clinical aspects

B.1 Surveillance study reporting incidences of occupational skin exposures

B.1.A Skin exposure major focus

D Hazard identification

D.1 Potential health effects resulting from specific chemicals

D.1.B Allergic contact dermatitis/sensitization

D.2 Summaries of health effects, dose-response relationships

This paper answers the following questions about occupational contact dermatitis (OCD):

1. What is its public health importance?

2. How large are prevalence and incidence?

3. What industries are associated with higher risk?

4. What are the occupational exposures that cause it?

5. Who is at risk?

6. What is the prognosis for patients?

7. What preventative measures and interventions are effective? 
Article ID:

Citation:

Resource type:

Educational materials:

Number of references:

Industries/occupations:

Specific process:

Chemical:

Specific chemicals:

Mixtures:

Audience:

Topics addressed:

Summary:
78

Diepgen TL, Coenraads PJ [1999]. The epidemiology of occupational contact dermatitis. Int Arch Occup Environ Health 72(8):496-506.

Journal article—review, meta-analysis

No

75

General—overview

Discussion of frequency of occupational contact dermatitis (OCD) among certain occupational categories.

Dichromate

No

Professional

A Overview

A.2 Health hazards resulting from skin exposure to chemicals

B Surveillance and clinical aspects

B.1 Surveillance study reporting incidences of occupational skin exposures

B.1.A Skin exposure major focus

D Hazard identification

D.1 Potential health effects resulting from specific chemicals

D.1.A Irritant contact dermatitis

D.1.B Allergic contact dermatitis/sensitization

D.3 Characterization protocols

D.3.B Irritation potential

D.3.C Sensitization potential

F Risk management

F.1 Exposure control strategies

F.1.D PPE and PPE rules

F.1.E Skin management, barrier creams, moisturizers, cleansers, and rubs

This review article discusses the lack of epidemiologic data on occupational contact dermatitis (OCD). It also discusses case ascertainment and bias, distribution of occupational allergic and irritant contact dermatitis, the interrelationship between exogenous (allergens, irritants) and endogenous factors, the prognosis of OCD, the 
social and economic impact of OCD, and the need for intervention studies.

Citation:

Resource type:

Educational materials:

Number of references:

Industries/occupations:

Specific process:

Chemical:

Specific chemicals:

Mixtures:

Audience:

Topics addressed:
Article ID:

\section{9}

Elsner P, Wigger-Alberti W [2003]. Skin-conditioning products in occupational dermatology. Int Arch Occup Environ Health 76(5):351-54.

Journal article—review, meta-analysis

No

20

hand cleansers

No

Professional

A Overview

A.4 Skin physiology and function as barriers to chemical insults

D Hazard identification

D.1 Potential health effects resulting from specific chemicals

D.1.A Irritant contact dermatitis

F Risk management

F.1 Exposure control strategies

F.1.D PPE and PPE rules

F.1.E Skin management, barrier creams, moisturizers, cleansers, and rubs

Summary:

This review discusses the chemistry and mode of action of moisturizers in their prevention of occupational contact dermatitis.

Article ID:

80

Citation:

Resource type:

Educational materials:
Emmett EA [2002]. Occupational contact dermatitis I: Incidence and return to work pressures. Am J Contact Dermat 13(1).

Journal article-review, meta-analysis

No 
Number of references:

8

Industries/occupations:

Specific process:

Chemical:

Specific chemicals:

Mixtures:

Audience:

Topics addressed:

Summary:

Article ID:

\section{Citation:}

\section{Resource type:}

Educational materials:

Number of references:

Industries/occupations:

Specific process:

Chemical:

Specific chemicals:

Mixtures:

Audience:

51
No

Professional

B Surveillance and clinical aspects

B.1 Surveillance study reporting incidences of occupational skin exposures

B.1.A Skin exposure major focus

B.2 Loss of workdays and impact on productivity

This is the first article in a two-part series. Both articles are summarized below:

Part 1 (ID 80): Describes changes in the incidence of recorded occupational skin disease from 1972 to 1999 and suggests explanations for periodic changes. It provides trends for some specific industries. The authors argue for a more sophisticated approach to prevention and management to reduce the burden of occupational skin disease.

Part 2 (ID 81): Presents the present state of risk assessment (including components of hazard identification, dermal exposure measurement, absorption, dose-response, and risk characterization) and the prognosis for occupational contact dermatitis.

\section{1}

Emmett EA [2003]. Occupational contact dermatitis II: Risk assessment and prognosis. Am J Contact Dermat 14(1).

Journal article_-review, meta-analysis

No

General-overview

General-overview

No

Professional 
Topics addressed:

Summary:
A Overview

A.3 Investigation, intervention, and control of occupational skin exposures

A.4 Skin physiology and function as barriers to chemical insults

B Surveillance and clinical aspects

B.1 Surveillance study reporting incidences of occupational skin exposures

B.1.A Skin exposure major focus

B.4 Clinical protocols for recognition of skin exposure health effects

C Exposure characterization

C.2 Description of factors influencing exposure conditions

C.2.B Exposure concentration

C.2.C Skin area affected

C.2.E Uptake

C.4 Direct methods to measure exposure

C.4.B Skin

C.4.C Biomonitoring

D Hazard identification

D.1 Potential health effects resulting from specific chemicals

D.1.A Irritant contact dermatitis

D.1.B Allergic contact dermatitis/sensitization

D.2 Summaries of health effects, dose-response relationships

E Risk assessment

E.1 Guidelines for risk assessment or analysis

21-30 E.1.A Localized health effects

This is the second article in a 2-part series. Both articles are summarized below:

Part 1 (ID 80): Describes changes in the incidence of recorded occupational skin disease from 1972 to 1999 and suggests explanations for periodic changes. It also examines trends for some specific industries. The authors argue for a more sophisticated approach to prevention and management to reduce the burden of occupational skin disease.

Part 2 (ID 81): Presents the present state of risk assessment (including components of hazard identification, dermal 
exposure measurement, absorption, dose-response, and risk characterization) and the prognosis for occupational contact dermatitis.

Article ID:

Citation:

Resource type:

Educational materials:

Number of references:

Industries/occupations:

Specific process:

Chemical:

Specific chemicals:

Mixtures:

Audience:

Topics addressed:

\section{2}

Enviroderm Services [2005]. Dermatological

engineering. [www.enviroderm.co.uk/].

Web site

Yes

General—overview

General—overview, coolants, latex

Metalworking fluids

No

Professional

A Overview

A.1 Occurrence of skin exposures in the workplace

A.2 Health hazards resulting from skin exposure to chemicals

A.4 Skin physiology and function as barriers to chemical insults

B Surveillance and clinical aspects

B.3 Surveillance study protocols/procedures for gathering data

B.4 Clinical protocols for recognition of skin exposure health effects

C Exposure characterization

C.1 Workplace factors associated with harmful skin exposures

C.2 Description of factors influencing exposure conditions

C.2.A Exposure intensity/frequency/duration

C.2.B Exposure concentration

C.2.C Skin area affected

C.2.E Uptake

C.4 Direct methods to measure exposure

C.4.B Skin

D Hazard identification 
Summary:

D.1 Potential health effects resulting from specific chemicals

D.1.A Irritant contact dermatitis

D.1.B Allergic contact dermatitis/sensitization

D.1.C Systemic toxicity

E Risk assessment

E.1 Guidelines for risk assessment or analysis

E.1.A Localized health effects

E.1.B Systemic health effects

E.2 Example of risk assessments

F Risk management

F.1 Exposure control strategies

F.1.A Substitution

F.1.B Engineering controls

F.1.C Work practice/Administrative controls

F.1.D PPE and PPE rules

F.1.E Skin management, barrier creams, moisturizers, cleansers, and rubs

F.2 Protocols for risk management

F.2.B Development of approach to achieve exposure reduction goal

Enviroderm Services is a U.K.-based consulting firm founded by Chris Packham, a recognized expert in the field of dermal exposure, that specializes in the workplace dermal exposures prevention and control. Although consulting services are limited to the U.K., they have available through their Web site a different dermal exposure-related materials, including literature, educational and training materials, workplace posters, skin-monitoring equipment, dermal risk assessment forms, and skin health surveillance tools including forms and questionnaires. The Web site also contains a brief description of all the materials and literature available for purchase.

Literature available through the Web site includes:

- Essentials of Occupational Skin Management. A 15-chapter textbook featured as a separate resource in this indexed dermal bibliography (see ID 169).

- Risk Assessment (for dermal exposure) Forms

- Technical Bulletin No.1 Skin Management

- Technical Bulletin No.2 Occupational Skin Diseases 
- Technical Bulletin No.3 Chemical protection using gloves

- Technical Bulletin No.4 Health Surveillance and the skin

- Technical Bulletin No.5 Irritant Contact Dermatitis

- Technical Bulletin No.6 Thoughts on Latex Allergy

- Technical Bulletin No.7 Personal Hygiene

- Technical Bulletin No.8 Is it occupational?

- Technical Bulletin No.9 Emollients

- Technical Bulletin No.10 Barrier Creams

- Technical Bulletin No.11 Risk Assessment for Dermal Exposure

- Technical Bulletin No.12 Risk management

- Technical Bulletin No.13 Allergic Skin Disorders

- Technical Bulletin No.14 Metalworking fluids

- Technical Bulletin No.15 Infection control and the skin

- Technical Bulletin No.16 Investigating a skin problem

- Technical Bulletin No.17 How hazardous is that chemical?

- Technical Bulletin No.18 Skin Exposure Measurement

Article ID:

Citation:

Resource type:

Educational materials:

Number of references:

Industries/occupations:

Specific process:

Chemical:

Specific chemicals:

Mixtures:

Audience:

Topics addressed:

\section{3}

Organisation for Economic Co-operation and Development (OECD) [1999]. OECD series on testing and assessment, number 16: detailed review document on classification systems for skin irritation/corrosion in OECD member countries. Paris, France: OECD, ENV/JM/ MONO(99)6.

Journal article—review, meta-analysis

No

22

No

Professional

A Overview

A.5 Dermal regulations and skin notations 
Summary:

Article ID:

Citation:

Resource type:

Educational materials:

Number of references:

Industries/occupations:

Specific process:

Chemical:

Specific chemicals:

Audience:

Topics addressed:
This report compares the dermal irritation/corrosion hazard classification procedures used in Canada, the US, OECD, European Union, and Norway. Issues requiring resolution are discussed.

\section{4}

OECD [2004]. OECD series on testing and assessment, number 28: Guidance document for the conduct of skin absorption studies. Paris, France: OECD, ENV/JM/MONO (2004)2.

Guideline

No

59
Mixtures:

No

Professional

C Exposure characterization

C.2 Description of factors influencing exposure conditions

C.2.E Uptake

D Hazard identification

D.3 Characterization protocols

D.3.E Measurement of skin permeation rates and reservoir effects

Summary:

The OECD Guidance Document for the Conduct of Skin Absorption Studies was published by the Organization for Economic Co-operation and Development (OECD), an intergovernmental organization which representatives 30 industrialized countries in North America, Europe, and the Pacific, as well as the European Commission. It provides guidance developed by OECD at Research Triangle Park, North Carolina, in October 1997 called "Percutaneous Absorption Methods as Test Guidelines" for in vitro and in vivo studies.
Article ID:

Citation:

\section{5}

USEPA [1998]. Harmonized test guidelines. [www.epa.gov/ opptsfrs/home/guidelin.htm]. 
Resource type:

Educational materials:

Number of references:

Industries/occupations:

Specific process:

Chemical:

Specific chemicals:

Mixtures:

Audience:

Topics addressed:

Summary:
Web page

No

General—overview, Agricultural

General—overview, pesticides

No

Professional

C Exposure characterization

C.4 Direct methods to measure exposure

C.4.A Surfaces

C.4.B Skin

D Hazard identification

D.3 Characterization protocols

D.3.B Irritation potential

D.3.C Sensitization potential

D.3.D Potential to cause systemic effects

D.3.E Measurement of skin permeation rates and reservoir effects

The USEPA Office of Prevention, Pesticides and Toxic Substances (OPPTS) harmonized test guidelines were developed to minimize variations in testing procedures under the Toxic Substances Control Act (TSCA) and the FIFRA. These were developed primarily for occupational pesticides and other toxic substances.

In Series 870, The Health Effects Test Guidelines, guidelines can be found for use in the testing of pesticides and toxic substances and the development of test data that must be submitted to the USEPA for review under federal regulations. The following dermal-related test guidelines can be found in this series:

- 870.1200 Acute dermal toxicity

- 870.2500 Acute dermal irritation

- 870.2600 Skin sensitization

- 870.3200 21/28-Day dermal toxicity

- 870.3250 90-Day dermal toxicity

In Series 875, The Occupational and Residential Exposure Test Guidelines: Post Application Exposure Guidelines, the following dermal exposure test guidelines can be found: 
- 875.1100 Dermal exposure-outdoor

- 875.1200 Dermal exposure-indoor

- 875.2400 Dermal exposure

Article ID:

Citation:

Resource type:

Educational materials:

Number of references:

Industries/occupations:

Specific process:

Chemical:

Specific chemicals:

Mixtures:

Audience:

Topics addressed:

\section{6}

USEPA [2000]. Summary report for the workshop on issues associated with dermal exposure and uptake. Washington, DC: U.S. Environmental Protection Agency, FRL-7032-8.

Technical publication/report

No

22

Waste Management

No

Professional

C Exposure characterization

C.2 Description of factors influencing exposure conditions

C.2.E Uptake

C.5 Exposure modeling

Summary:
This paper is a summary of a December 1998 workshop that discussed issues concerning dermal uptake, permeability, and absorbed dose to chemicals.
Article ID:

Citation:

Resource type:

Educational materials:

Number of references:

Industries/occupations:

Specific process:

Chemical:

Specific chemicals:

\section{7}

USEPA [2005]. [www.epa.gov].

Web site

No

General—overview, Agricultural, Manufacturing autobody painting

General—overview, pesticides, petroleum products \& lubricants, solvents 
Mixtures:

Audience:

Topics addressed:
No

Professional

B Surveillance and clinical aspects

B.1 Surveillance study reporting incidences of occupational skin exposures

B.1.A Skin exposure major focus

B.2 Loss of workdays and impact on productivity

B.3 Surveillance study protocols/procedures for gathering data

C Exposure characterization

C.2 Description of factors influencing exposure conditions

C.2.A Exposure intensity/frequency/duration

C.2.E Uptake

C.4 Direct methods to measure exposure

C.4.A Surfaces

C.4.B Skin

C.4.C Biomonitoring

C.5 Exposure modeling

D Hazard identification

D.3 Characterization protocols

D.3.B Irritation potential

D.3.C Sensitization potential

D.3.D Potential to cause systemic effects

D.3.E Measurement of skin permeation rates and reservoir effects

E Risk assessment

E.1 Guidelines for risk assessment or analysis

E.1.A Localized health effects

E.1.B Systemic health effects

E.2 Example of risk assessments

F Risk management

F.1 Exposure control strategies

F.1.A Substitution

F.1.C Work practice/Administrative controls

F.1.D PPE and PPE rules

The USEPA is the federal agency tasked with protecting human health and the environment. In addition to developing and enforcing regulations, the USEPA also 
performs environmental research, sponsors voluntary partnerships and programs, advances environmental education, and publishes information associated with the environment. Although the USEPA's work deals more with environmental exposures than occupational exposures (except in the case of pesticides), some resources are designed for occupational settings and some are applicable to both. The USEPA has produced resources related to dermal exposure to chemicals that can be found on their Web site, including:

- Series 875, Occupational and Residential Exposure Test Guidelines: Post Application Exposure Guidelines, Group B. These guidelines provide background information on the application of exposure monitoring test guidelines, dermal exposure (outdoor), dermal exposure (indoor), biological monitoring, and data reporting and calculations.

- Cleaner Technologies Substitutes Assessment: A Methodology and Resource Guide. This contains guidelines on evaluating chemical substitution, though not specific to dermal exposures. Recommendations that may be applicable can be found here.

- "Choosing the Right Gloves for Painting Cars." This covers how to select chemical resistant gloves for automobile paint work.

- Dermal Exposure Assessment: Principles and Applications. This 1992 guidance document covers the principles of dermal absorption and outlines procedures on how to apply these principles to actual dermal exposure assessments involving contact with chemical vapors, air, soil and water.

- Summary Report for the Workshop on Issues Associated With Dermal Exposure and Uptake. This is a summary from a 1998 workshop that discussed technical issues associated with dermal exposure and risk assessment.

- Exposure Factors Handbook, Chapter 6, "Dermal." This 1997 document on general dermal exposure considerations is directed at environmental exposures, but may apply to some occupational settings as well.

Article ID:

Citation:

\section{8}

European Agency for Safety and Health and Work [2005]. European Agency for Safety and Health and Work. [http:// europe.osha.eu.int/OSHA]. 
Resource type:

Educational materials:

Number of references:

Industries/occupations:

Specific process:

Chemical:

Specific chemicals:

Mixtures:

Audience:

Topics addressed:

Summary:
Web site

No

General—overview

General—overview

No

General

A Overview

A.1 Occurrence of skin exposures in the workplace

A.2 Health hazards resulting from skin exposure to chemicals

A.3 Dermal regulations and skin notations

B Exposure characterization

B.1 Job/tasks, industries/processes, or chemicals associated with skin exposures

C Hazard identification

C.2 Tables/charts/lists of hazards for specific chemicals

E Risk management

E.3 "Best practices"/guidelines/recommendations

E.3.A Substitution

E.3.B Engineering controls

E.3.C Work practice/administration controls

E.3.D PPE and PPE rules

E.3.E Skin management, barrier creams, moisturizers, cleansers, and rubs

The European Agency for Safety and Health at Work collects, analyzes, and promotes occupational safetyand health-related information in Europe. The agency is a tripartite European Union organization that brings together representatives from governments, employers' and workers' organizations, as well as from the European Commission. The agency's web portal provides links to over 30 national Web sites, usually the lead OSH organization in the European Union. member states, candidate countries, and other international partners. Information on dermal exposure can be found here based on chemical, risks, industry or sector, and topics of interest. Information is also available in multiple languages. 
Useful information on dermal exposures available through the agency's Web site includes the following fact sheets:

- Issue 34, Eliminating and Substituting Dangerous Substances

- Issue 35, Communicating Information about Dangerous Substance

- Issue 40, Skin Sensitizers

Article ID:

Citation:

Resource type:

Educational materials:

Number of references:

Industries/occupations:

Specific process:

Chemical:

Specific chemicals:

Mixtures:

Audience:

Topics addressed:

\section{9}

European Center for Ecotoxicity and Toxicology of Chemicals (ECETOC) [1999]. Skin and respiratory sensitisers: Reference chemical data bank. Brussels, Belgium: ECETOC, Technical Report No. 77.

Technical publication/report

No

292

No

Professional

C Exposure characterization

C.5 Exposure modeling

D Hazard identification

D.1 Potential health effects resulting from specific chemicals

D.1.A Irritant contact dermatitis

D.1.B Allergic contact dermatitis/sensitization

This paper provides a list of skin and respiratory sensitizers which may be used for the validation of in vivo or in vitro toxicological tests. The list also identifies chemicals that will facilitate the evaluation and validation of proposed predictive test methods for skin and/or respiratory sensitization potential. It documents those chemicals that are recommended for use as positive and negative controls in the assessment of new predictive tests for skin or respiratory sensitization potential and assesses the utility and accuracy of "novel" test methods. 
Article ID:

Citation:

Resource type:

Educational materials:

Number of references:

Industries/occupations:

Specific process:

Chemical:

Specific chemicals:

Mixtures:

Audience:

Topics addressed:
90

Fehrenbacher C, Arnold F, Marquart H [2003]. Approaches for occupational dermal exposure assessment and management. In: DiNardi SR, ed. The occupational environment: its evaluation, control, and management. 2nd ed. Fairfax, VA: AIHA.

Book/monograph, chapter

No

53

2,4-dichlorophenol

No

Professional

C Exposure characterization

C.2 Description of factors influencing exposure conditions

C.2.A Exposure intensity/frequency/duration

C.2.B Exposure concentration

C.2.C Skin area affected

C.2.E Uptake

C.4 Direct methods to measure exposure

C.4.A Surfaces

C.4.B Skin

C.4.C Biomonitoring

C.5 Exposure modeling

E Risk assessment

E.1 Guidelines for risk assessment or analysis

E.1.A Localized health effects

E.1.B Systemic health effects

F Risk management

F.1 Exposure control strategies

F.1.A Substitution

F.1.B Engineering controls

F.1.C Work practice/Administrative controls

F.1.D PPE and PPE rules

F.1.E Skin management, barrier creams, moisturizers, cleansers, and rubs 
Summary:

Article ID:

Citation:

Resource type:

Educational materials:

Number of references:

Industries/occupations:

Specific process:

Chemical:

Specific chemicals:

Mixtures:

Audience:

Topics addressed:
Chapter 17, "Approaches for Occupational Dermal Exposure Assessment and Management," from the AIHA book The Occupational Environmental: Its Evaluation Control and Management (the White Book). This chapter discusses dermal exposure monitoring methods, the process of dermal absorption, methods to measure dermal uptake, a tiered approach to performing dermal exposure assessments, and the control and management of occupational dermal exposures.

\section{1}

Fenske RA [1993]. Dermal exposure assessment techniques. Ann Occup Hyg 37(6):687-706.

Journal article-review, meta-analysis

No

94

No

Professional

C Exposure characterization

C.2 Description of factors influencing exposure conditions

C.2.A Exposure intensity/frequency/duration

C.2.B Exposure concentration

C.2.C Skin area affected

C.2.E Uptake

C.4 Direct methods to measure exposure

C.4.A Surfaces

C.4.B Skin

C.4.C Biomonitoring

C.5 Exposure modeling

Summary:

This article discusses three primary pathways to exposure: immersion, deposition of aerosol or uptake of vapor through the skin, and contact with contaminated surfaces. It also discusses three primary sampling methods: surrogate skin, chemical removal, and fluorescent tracers. This article also presents a dermal exposure sampling strategy which addresses issues associated with the 
sampling method, representative sampling, and sample duration. Finally, it recommends the development of dermal occupational exposure limits (DOELs) for selected workplaces and chemical agents.

Article ID:

\section{2}

\section{Citation:}

Resource type:

Educational materials:

Number of references:

Industries/occupations:

Specific process:

Chemical:

Specific chemicals:

Mixtures:

Audience:

Topics addressed:

Summary:

Article ID:

Citation:

Resource type:

Educational materials:

Number of references:

Industries/occupations:

Specific process:

Chemical:

Specific chemicals:

Mixtures:

Audience:

Topics addressed:
Fenske RA [2000]. Dermal exposure: A decade of real progress. Ann Occup Hyg 44(7):489-91.

Other-editorial

No

17

No

Professional

A Overview

A.3 Investigation, intervention, and control of occupational skin exposures

This letter to the editor introduces an issue relevant to the journal which features recent work on workplace dermal exposure assessment. It summarizes the history of progress made in dermal exposure assessment through 2000.

\section{3}

Fitzpatrick D, Corish J, Hayes B [2004]. Modeling skin permeability in risk assessment-the future. Chemosphere 55(10):1309-14.

Journal article_-review, meta-analysis

No

31

No

Professional

C Exposure characterization 
Summary:

Article ID:

Citation:

Resource type:

Educational materials:

Number of references:

Industries/occupations:

Specific process:

Chemical:

Specific chemicals:

Mixtures:

Audience:

Topics addressed:

Summary:
C.2 Description of factors influencing exposure conditions

C.2.E Uptake

C.5 Exposure modeling

D Hazard identification

D.3 Characterization protocols

D.3.E Measurement of skin permeation rates and reservoir effects

D.3.F QSARs—development, validation, and application

This article presents recent progress in skin permeability modeling and compares two methods of assessing skin permeability: quantitative structure-activity relationships (QSARs) and mathematical modeling based on analytical or numerical solutions to partition and transport equations. It also proposes steps that can be taken for future advancements in this field.

\section{4}

Flynn MR, Koto Y, Fent K, Nylander-French LA [2006]. Modeling dermal exposure - an illustration for spray painting applications. J Occup Environ Hyg 3(9):475-80. Journal article_-primary

No

15

Construction, Automotive

Spray painting, Autobody workers

No

Professional

C Exposure characterization

C.5 Exposure modeling

This study presents a model to ascertain dermal exposure from aerosol spray paint deposition on human forearm hair.
Article ID:

Citation:

\section{5}

Forsberg K, Mansdorf SZ [2002]. Quick selection guide to CPC, 4th ed. New York: J. Wiley. 
Resource type:

Educational materials:

Number of references:

Industries/occupations:

Specific process:

Chemical:

Specific chemicals:

Mixtures:

Audience:

Topics addressed:

Summary:
Brochure, pamphlet

Yes

0
Index includes over 700 chemicals

No

General

A Overview

A.3 Dermal regulations and skin notations

C Hazard identification

C.1 Risk phrases, hazard symbols, skin designations

C.2 Tables/charts/lists of hazards for specific chemicals

E Risk management

E.3 "Best practices"/guidelines/recommendations

E.3.D PPE and PPE rules

This pocket-sized field guide for spill responders, safety engineers, industrial hygienists, chemists and chemical engineers, and other workers presents information on 700 chemicals, additional synonyms, CAS numbers, risk codes, and special notations to alert the user. It also discusses 16 PPE barrier materials used.

\section{CONTENTS}

1. Introduction

2. Selection and Use of CPC.

3. Chemical Index. Contains the chemical class numbers, chemical names and synonyms, chemical abstract service (CAS) numbers, risk codes, and special "skin" and "caution" notations.

4. Selection Recommendations. Provides color coded recommendations organized numerically by chemical class based on 11,000 permeation and 3,000 degradation test data.

5. Glossary

6. Standards for CPC

7. Manufacturers of CPC. Includes the names, addresses, and phone numbers of the suppliers and manufacturers of the CPC materials. 
Article ID:

Citation:

Resource type:

Educational materials:

Number of references:

Industries/occupations:

Specific process:

Chemical:

Specific chemicals:

Mixtures:

Audience:

Topics addressed:

Summary:
96

Furtaw EJ Jr [2001]. An overview of human exposure modeling activities at the USEPA's National Exposure Research Laboratory (NERL). Toxicol Ind Health 17:302-14.

Journal article—primary

No

18

No

Professional

C Exposure characterization

C.5 Exposure modeling

E Risk assessment

E.2 Example of risk assessments

This article reviews the following risk assessment models developed by the USEPA's NERL. NERL modeling efforts, though directed at environmental exposures, are applicable to occupational exposures as well. Modeling has focused on understanding the factors that influence exposure and has been designed for use in risk assessments and for risk management. Specific models reviewed include:

- Community Multiscale Air Quality (CMAQ) Model (Models-3/CMAQ) for pollutant concentrations in ambient (outdoor) air.

- Computational fluid dynamics (CFD) for air flow and pollutant concentrations.

- Stochastic Human Exposure and Dose Simulation (SHEDS) Model for human inhalation exposure to airborne particulates, toxics, or pesticides.

- Framework for Risk Analysis in Multimedia Environmental Systems-Multimedia, Multipathway, Multireceptor Risk Assessment (FRAMES-3MRA) for human and ecological exposure and risk assessments of hazardous waste sites.

- Exposure-Related Dose-Estimating Model (ERDEM) for physiologically based pharmacokinetic (PBPK) modeling of pesticides and VOCs. 
Article ID: $\quad 97$

Citation:

Resource type:

Educational materials:

Number of references:

Industries/occupations:

Specific process:

Chemical:

Specific chemicals:

Mixtures:

Audience:

Topics addressed:

Summary:

Article ID:

Citation:

Resource type:

Educational materials:

Number of references:
Garrod AN, Rajan-Sithamparanadarajah R [2003].

Developing COSHH essentials: Dermal exposure, personal protective equipment and first aid. Ann Occup Hyg 47(7):577-88.

Journal article—review, meta-analysis

No

20

No

Professional

A Overview

A.5 Dermal regulations and skin notations

C Exposure characterization

C.2 Description of factors influencing exposure conditions

C.2.A Exposure intensity/frequency/duration

C.2.B Exposure concentration

C.2.E Uptake

C.5 Exposure modeling

This paper discusses how to apply COSHH Essentials, originally developed in the U.K. to control inhalation exposures in the workplace and to control dermal exposures. It examines the factors affecting skin exposure, and outlines options to band chemical hazards for emergency planning according to a minimum of information, i.e., the danger symbol on a product label. It also discusses dermal hazard classifications.
98

Geer LA, Anna D, Curbow B, ener-West M, de Joode BW, Mitchell C, Buckley TJ [2007]. Survey assessment of worker dermal exposure and underlying behavioral determinants. J Occup Environ Hyg 4(11):809-20.

Journal article—primary

No

34 
Industries/occupations:

Specific process:

Chemical:

Specific chemicals:

Mixtures:

Audience:

Topics addressed:

Summary:
No

Professional

F Risk management

F.1 Exposure control strategies

F.1.C Work practice/Administrative controls

F.1.D PPE and PPE rules

This study assesses worker knowledge, attitudes, and perceptions of workplace dermal hazards using a questionnaire, compares worker and manager scores, evaluates worker dermal exposure using DREAM, and identifies potential behavioral factors underlying exposure.

Article ID:

99

Citation:

Resource type:

Educational materials:

Number of references:

Industries/occupations:

Specific process:

Chemical:

Specific chemicals:

Mixtures:

Audience:

Topics addressed:
Gerberick GF, Ryan CA, Kern PS, Dearman RJ, Kimber I, Patlewicz GY, Basketter DA [2004]. A chemical dataset for evaluation of alternative approaches to skin-sensitization testing. Contact Dermatitis 50(5):274-88.

Journal article-review, meta-analysis

No

45

\section{Summary:}

No

Professional

D Hazard identification

D.1 Potential health effects resulting from specific chemicals

D.1.B Allergic contact dermatitis/sensitization

D.3 Characterization protocols

D.3.C Sensitization potential

This article presents a list of 244 chemicals and their relative sensitization potency, as determined by the local lymph node assay (LLNA). The authors state this dataset 
can be used to evaluate and calibrate novel approaches to skin sensitization testing.

Article ID:

\section{Citation:}

Resource type:

Educational materials:

Number of references:

Industries/occupations:

Specific process:

Chemical:

Specific chemicals:

Mixtures:

Audience:

Topics addressed:

Summary:

\section{0}

Goede HA, Tijssen SC, Schipper HJ, Warren N, Oppl R, Kalberlah F, van Hemmen JJ [2003]. Classification of dermal exposure modifiers and assignment of values for a risk assessment toolkit. Ann Occup Hyg 47(8):609-18.

Journal article—review, meta-analysis

No

39

No

Professional

E Risk assessment

E.1 Guidelines for risk assessment or analysis

E.1.A Localized health effects

E.1.B Systemic health effects

This article is the 4th article of a six-part series on RISKOFDERM, a tool for conducting risk assessments. The series was published in the Annals of Occupational Hygiene in 2003. The following briefly summarizes each paper in the series:

1. ID 212-Outlines a "toolkit" for conducting dermal occupational risk assessments.

2. ID 163-Describes the assumptions in the toolkit and describes an approach to exposure assessment used by the toolkit.

3. ID 139-Describes the determinants relevant for dermal exposure models in the scope of regulatory risk assessment.

4. ID 219-Describes how default dermal exposure values can be adjusted for specific work situations.

5. ID 100-Describes the derivation of the toolkit's default task-based dermal exposure values.

6. ID 193-Describes the development of "intrinsic toxicity" (IT) scores used for hazard characterization. 
Article ID:

Citation:

Resource type:

Educational materials:

Number of references:

Industries/occupations:

Specific process:

Chemical:

Specific chemicals:

Mixtures:

Audience:

Topics addressed:
101

Graves CG, Matanoski GM, Tardiff RG [2000]. Carbonless copy paper and workplace safety: a review. Regul Toxicol Pharmacol 32(1):99-117.

Journal article—review, meta-analysis

No

122

Cleaning agents, PCBs, other: carbonless copy paper (CCP) Formaldehyde

No

Professional

A Overview

A.1 Occurrence of skin exposures in the workplace

A.2 Health hazards resulting from skin exposure to chemicals

B Surveillance and clinical aspects

B.1 Surveillance study reporting incidences of occupational skin exposures

B.1.A Skin exposure major focus

D Hazard identification

D.1 Potential health effects resulting from specific chemicals

D.1.A Irritant contact dermatitis

D.3 Characterization protocols

D.3.B Irritation potential

D.3.C Sensitization potential

This paper presents a meta-analysis of 121 papers published on CCP since 1987. CCP has been alleged to cause skin irritation, however, this weight-of-evidence analysis concludes that no irritation or sensitization from CCP should be expected under normal conditions of manufacture and use.
Article ID:

Citation:

\section{2}

University of California, San Diego [1998]. Prediction and assessment of dermal exposure. La Jolla, CA: University of California, San Diego, AD-a358 903.1998. 
Resource type:

Educational materials:

Number of references:

Industries/occupations:

Specific process:

Chemical:

Specific chemicals:

Mixtures:

Audience:

Topics addressed:

Summary:
Technical publication/report

No

13
No

Professional

C Exposure characterization

C.2 Description of factors influencing exposure conditions

C.2.E Uptake

C.5 Exposure modeling

This paper presents the basis for algorithms developed to predict the rate of absorption of chemicals following dermal exposure. These algorithms are necessary for performing risk assessments. The paper includes the results of a literature review as well as the algorithm test results.

Article ID:

103

Citation:

Resource type:

Educational materials:

Number of references:

Industries/occupations:

Specific process:

Chemical:

Specific chemicals:

Mixtures:

Audience:

Topics addressed:
Hamann CP, DePaola LG, Rodgers PA [2005].

Occupation-related allergies in dentistry. J Am Dent Assoc 136(4):500-10.

Journal article—review, meta-analysis

No

73

Dentistry

Latex, plastics and resins, rubber additives, other: adhesives, antiseptics, artificial fingernails, dental bonding agents, disinfectants, equipment sterilization solutions, skin care products, rubber gloves, radiographic and photo chemical

No

Professional

A Overview

A.1 Occurrence of skin exposures in the workplace 
Summary:

A.2 Health hazards resulting from skin exposure to chemicals

B Surveillance and clinical aspects

B.1 Surveillance study reporting incidences of occupational skin exposures

B.1.A Skin exposure major focus

D Hazard identification

D.1 Potential health effects resulting from specific chemicals

D.1.A Irritant contact dermatitis

D.1.B Allergic contact dermatitis/sensitization

F Risk management

F.1 Exposure control strategies

F.1.A Substitution

F.1.B Engineering controls

F.1.C Work practice/Administrative controls

F.1.D PPE and PPE rules

F.1.E Skin management, barrier creams, moisturizers, cleansers, and rubs

This paper presents the health effects associated with occupation-related allergies in dentistry. Natural rubber latex (NRL) protein allergy, allergic contact dermatitis, and irritant contact dermatitis are discussed. Topics include diagnosis, exposure measuring, management and prevention, and some surveillance information.

Article ID:

104

Citation:

Resource type:

Educational materials:

Number of references:

Industries/occupations:

Specific process:

Chemical:

Specific chemicals:

Mixtures:

Audience:
Hatch KL, Maibach HI [2000]. Textile dye allergic contact dermatitis prevalence. Contact Dermatitis 42(4):187-95.

Journal article-review, meta-analysis

No

20

Manufacturing

Organic dyes

Paper examines over 60 dyes for prevalence data including disperse orange 3, yellow 3, red 1, blue 124, black 1, brown 1 , and reactive green 12 .

No

Professional 
Topics addressed:

Summary:
B Surveillance and clinical aspects

B.1 Surveillance study reporting incidences of occupational skin exposures

B.1.A Skin exposure major focus

C Exposure characterization

C.4 Direct methods to measure exposure

C.4.B Skin

This paper summarizes textile dye allergic contact dermatitis prevalence studies and makes recommendations for future work.

Article ID:

105

Citation:

Resource type:

Educational materials:

Number of references:

Industries/occupations:

Specific process:

Chemical:

Specific chemicals:

Mixtures:

Audience:

Topics addressed:
HSE [2005]. Skin at work. [www.hse.gov.uk/skin/].

Web page

No

General-overview, Beauty/Cosmetology, Manufacturing-Chemical, Manufacturing-Other, Service-Food

Hairdressers, catering, printing

General-overview, food products, heavy metals/inorganic compounds, latex, nanoparticles, pesticides, plastics and resins, $\mathrm{PCBs}$, soaps and detergents, solvents

Isocyanates, epoxy resins

No

Professional

A Overview

A.1 Occurrence of skin exposures in the workplace

A.2 Health hazards resulting from skin exposure to chemicals

A.3 Investigation, intervention, and control of occupational skin exposures

A.4 Skin physiology and function as barriers to chemical insults

B Surveillance and clinical aspects

B.1 Surveillance study reporting incidences of occupational skin exposures

B.1.A Skin exposure major focus

B.2 Loss of workdays and impact on productivity 
B.4 Clinical protocols for recognition of skin exposure health effects

C Exposure characterization

C.1 Workplace factors associated with harmful skin exposures

C.2 Description of factors influencing exposure conditions

C.2.C Skin area affected

C.4 Direct methods to measure exposure

C.4.A Surfaces

C.4.B Skin

C.4.C Biomonitoring

D Hazard identification

D.1 Potential health effects resulting from specific chemicals

D.1.A Irritant contact dermatitis

D.1.B Allergic contact dermatitis/sensitization

D.2 Summaries of health effects, dose-response relationships

D.3 Characterization protocols

D.3.A Corrosivity

D.3.C Sensitization potential

D.3.E Measurement of skin permeation rates and reservoir effects

E Risk assessment

E.1 Guidelines for risk assessment or analysis

E.1.A Localized health effects

E.1.B Systemic health effects

E.2 Example of risk assessments

F Risk management

F.1 Exposure control strategies

F.1.B Engineering controls

F.1.C Work practice/Administrative controls

F.1.D PPE and PPE rules

F.1.E Skin management, barrier creams, moisturizers, cleansers, and rubs

Summary:

Britain's Health and Safety Commission (HSC) and HSE are responsible for the regulation of occupational health and safety risks arising in the U.K. Any regulatory information provided here is specific to the U.K. This Web 
site provides comprehensive health and safety information, with most dermal exposure-related information found on the topic page Skin at Work.

The HSE's Skin at Work Web page includes a variety of free leaflets, including: Preventing Dermatitis at Work, Advice for Employers and Employees; MS24-Medical Aspects of Occupational Skin Disease; Managing Health and Safety topics, Personal Protective Equipment (PPE), Risk Assessment, and a number of chemical-specific leaflets. It also contains links to chemical-specific alert notices. The Skin at Work Web page provides specific dermal exposure information for the following industries: hairdressing, catering, and printing.

The HSE Web site also has information on different dermal exposure research topics, including:

- Contact dermatitis

- Occupational dermatitis

- Skin disease surveillance data

- Development of a method to assess biologically relevant dermal exposure

- Dermal exposure resulting from liquid contamination

- In vitro dermal absorption of liquids

- Health effects of particles produced for nanotechnologies

- Pesticides in air and/or on surfaces

- Draft guidelines on route-to-route extrapolation of toxicity data when assessing health risks of chemicals

- Development of a field method for the assessment of the effectiveness of barrier creams in preventing skin irritation reactions

- An assessment of skin sensitization by the use of epoxy resin in the construction industry

Article ID:

106

\section{Citation:}

Resource type:

Educational materials:

Number of references:

Industries/occupations:

Specific process:
Hewett P [2001]. Misinterpretation and misuse of exposure limits. Appl Occup Environ Hyg 16(2):251-56. Journal article-review, meta-analysis

No

28 
Chemical:

Specific chemicals:

Mixtures:

Audience:

Topics addressed:

Summary:

Article ID:

Citation:

Resource type:

Educational materials:

Number of references:

Industries/occupations:

Specific process:

Chemical:

Specific chemicals:

Mixtures:

Audience:

Topics addressed:

Summary:
No

Professional

A Overview

A.5 Dermal regulations and skin notations

E Risk assessment

E.1 Guidelines for risk assessment or analysis

E.1.A Localized health effects

E.1.B Systemic health effects

F Risk management

F.2 Protocols for risk management

F.2.B Development of approach to achieve exposure reduction goal

This article discusses occupational exposure limits (OELs) and distinguishes between how they should be used in risk assessment and exposure risk management and how they can be misused in each.

\section{7}

Hostynek JJ [2003]. Factors determining percutaneous metal absorption. Food Chem Toxicol 41(3):327-45.

Journal article—review, meta-analysis

No

157

Heavy metals/inorganic compounds

No

Professional

C Exposure characterization

C.2 Description of factors influencing exposure conditions

C.2.E Uptake

This review article describes factors affecting the permeability of metals through the skin including dose, vehicle, volume, counter ion, chemical bond and polarity, valence, protein reactivity, solubility, age of skin, 
anatomical site, homeostatic controls, skin layers, and oxidation/reduction.

Article ID:

Citation:

Resource type:

Educational materials:

Number of references:

Industries/occupations:

Specific process:

Chemical:

Specific chemicals:

Mixtures:

Audience:

Topics addressed:

Summary:
108

USEPA [1998]. Dermal and non-dietary ingestion exposure workshop: NERL Human Exposure Research Program. Research Triangle Park, NC: USEPA, NERL, Human Exposure \& Atmospheric Sciences Division, Human Exposure Analysis Branch.

Technical publication/report

No

125

pesticides

No

Professional

C Exposure characterization

C.2 Description of factors influencing exposure conditions

C.2.A Exposure intensity/frequency/duration

C.2.B Exposure concentration

C.2.C Skin area affected

C.2.E Uptake

C.4 Direct methods to measure exposure

C.4.A Surfaces

C.4.B Skin

C.4.C Biomonitoring

C.5 Exposure modeling

This paper presents a summary of the dermal and nondietary ingestion exposure workshop sponsored by USEPA NERL on September 17, 1998. The workshop evaluated methods for measuring and assessing children's exposures to pesticides via dermal contact with contaminated surfaces and objects, and through nondietary ingestion. The workshop also evaluated methods for characterizing concentrations of pesticides on surfaces and quantifying the transfer of contaminants to the skin surface or mouth. The workshop's objectives 
included identification of exposure assessment methods, determination of best approach, and evaluation of these methods' and approach's strengths and weaknesses. Dermal assessment methods reviewed include the microactivity approach, the macroactivity approach, biomonitoring, passive dosimetry, environmental exposure and activity pattern, florescent tracer, and dermal wash/rinse/wipe.

Article ID:

Citation:

Resource type:

Educational materials:

Number of references:

Industries/occupations:

Specific process:

Chemical:

Specific chemicals:

Mixtures:

Audience:

Topics addressed:

Summary:

\section{9}

Human Exposure Research Organisations Exchange (HEROX) [2005]. [www.herox.org/].

Web site

No

General-overview

General-overview

No

Professional

E Risk assessment

E.1 Guidelines for risk assessment or analysis

E.1.A Localized health effects

E.1.B Systemic health effects

HEROX is a forum for people interested in research on human exposure to hazardous substances. It provides information related to exposure to carcinogens, dermal exposure assessment, development of analytical methods, and exposure modeling research as well as access to databases on workplace exposure. Material on this site is edited by the Department of Environmental and Occupational Medicine at the University of Aberdeen, UK.
Article ID:

Citation:

Resource type:

Educational materials:

Number of references:

\section{0}

Interagency Coordinating Committee on the Validation of Alternative Methods (ICCVAM) [2005]. [http://iccvam. niehs.nih.gov].

Web site

No 
Industries/occupations:

Specific process:

Chemical:

Specific chemicals:

Mixtures:

Audience:

Topics addressed:

Summary:
General-overview

No

Professional

D Hazard identification

D.1 Potential health effects resulting from specific chemicals

D.1.B Allergic contact dermatitis/sensitization

D.3 Characterization protocols

D.3.A Corrosivity

D.3.B Irritation potential

D.3.C Sensitization potential

ICCVAM was established by the Director of the National Institute of Environmental Health Sciences (NIEHS) to implement NIEHS directives to develop and validate new test methods and to establish criteria and processes for the validation and regulatory acceptance of toxicological testing methods. To date, the following dermal assays and associated documents were submitted to ICCVAM for review and evaluation:

- Dermal corrosivity and irritation assays: Corrositex ${ }^{\circledR}$ Assay; EpiDerm ${ }^{\mathrm{TM}}$, EpiSkin ${ }^{\mathrm{TM}}$, and Rat Skin Transcutaneous Electrical Resistance (TER) Assay

- Murine Local Lymph Node Assay (LLNA) - a test method for assessing the allergic contact dermatitis potential of chemicals/compounds
Article ID:

Citation:

Resource type:

Educational materials:

Number of references:

Industries/occupations:

Specific process:

Chemical:

Specific chemicals:

Mixtures:

\section{1}

International Brotherhood of Teamsters [2006]. [www.teamster.org/].

Web site

No

General-overview

General-overview, plastics and resins, solvents

Diisocyanates

No 
Audience:

Topics addressed:

Summary:
General

A Overview

A.1 Occurrence of skin exposures in the workplace

A.2 Health hazards resulting from skin exposure to chemicals

B Exposure characterization

B.1 Job/tasks, industries/processes, or chemicals associated with skin exposures

E Risk management

E.1 Overview of skin exposure control options

The International Brotherhood of Teamsters' Safety and Health Department has a Web page on the Teamsters' Web site that contains information on health and safety issues associated with Teamster work activities. A variety of fact sheets in the health and safety section of the Teamster Web site address dermal exposure related issues, including:

- Dermatitis

- Diisocyanates

- Solvents

- General requirements for sanitation
Article ID:

\section{Citation:}

Resource type:

Educational materials:

Number of references:

Industries/occupations:

Specific process:

Chemical:

\section{2}

International Labour Organization (ILO), [2005]. [www.ilo.org/].

Web site

Yes

General_overview, Agricultural, Beauty/Cosmetology, Cleaning/Janitorial/Maid, Construction, Forestry/Fisheries, Manufacturing-Chemical, Medical Services, Mining, Service-Food, Service-Medical, Transportation/ Communications/Utility

General-overview, abrasives, cleaning agents, coolants, corrosives, fiberglass and other fibers, food products, hand cleansers, heavy metals/inorganic compounds, latex, nanoparticles, organic dyes, particulates, pesticides, petroleum products \& lubricants, plastics and resins, PAHs, PCBs, rubber additives, soaps and detergents, solvents

Specific chemicals: 


\begin{tabular}{|c|c|c|}
\hline \multirow{3}{*}{$\begin{array}{l}\text { Mixtures: } \\
\text { Audience: } \\
\text { Topics addressed: }\end{array}$} & \multicolumn{2}{|l|}{ Yes } \\
\hline & \multicolumn{2}{|c|}{ Professional } \\
\hline & A & Overview \\
\hline & A. 1 & Occurrence of skin exposures in the workplace \\
\hline & A. 2 & $\begin{array}{l}\text { Health hazards resulting from skin exposure to } \\
\text { chemicals }\end{array}$ \\
\hline & A. 3 & $\begin{array}{l}\text { Investigation, intervention, and control of } \\
\text { occupational skin exposures }\end{array}$ \\
\hline & A. 4 & $\begin{array}{l}\text { Skin physiology and function as barriers to } \\
\text { chemical insults }\end{array}$ \\
\hline & A. 5 & Dermal regulations and skin notations \\
\hline & $\mathrm{B}$ & Surveillance and clinical aspects \\
\hline & B.1 & $\begin{array}{l}\text { Surveillance study reporting incidences of } \\
\text { occupational skin exposures }\end{array}$ \\
\hline & B.1.A & Skin exposure major focus \\
\hline & B.1.B & Skin exposure minor focus \\
\hline & B. 2 & Loss of workdays and impact on productivity \\
\hline & $\mathrm{C}$ & Exposure characterization \\
\hline & C.1 & $\begin{array}{l}\text { Workplace factors associated with harmful skin } \\
\text { exposures }\end{array}$ \\
\hline & C. 2 & $\begin{array}{l}\text { Description of factors influencing exposure } \\
\text { conditions }\end{array}$ \\
\hline & C.2.A & Exposure intensity/frequency/duration \\
\hline & C.2.B & Exposure concentration \\
\hline & C.2.C & Skin area affected \\
\hline & $\mathrm{D}$ & Hazard identification \\
\hline & D.1 & $\begin{array}{l}\text { Potential health effects resulting from specific } \\
\text { chemicals }\end{array}$ \\
\hline & D.1.A & Irritant contact dermatitis \\
\hline & D.1.B & Allergic contact dermatitis/sensitization \\
\hline & D.1.C & Systemic toxicity \\
\hline & D.1.E & Contribution to overall exposure \\
\hline & $\mathrm{F}$ & Risk management \\
\hline & F.1 & Exposure control strategies \\
\hline & F.1.A & Substitution \\
\hline & F.1.B & Engineering controls \\
\hline & F.1.C & Work practice/Administrative controls \\
\hline & F.1.D & PPE and PPE rules \\
\hline
\end{tabular}




\section{Summary:}

F.1.E Skin management, barrier creams, moisturizers, cleansers, and rubs

The ILO is a specialized agency of the United Nations that promotes internationally recognized human and labor rights. Among other things, it provides technical assistance in the field of occupational safety and health. A variety occupational safety and health resources containing information on dermal exposure are available through this Web site. Key resources available through ILO include:

- International Occupational Safety and Health Information Centre (CIS): CIS was established in 1959 with the aim of facilitating the exchange of information about occupational safety and health being published around the world, regardless of the format or the language. A variety databases and services that contain dermal exposure information can be accessed through this center (http://www.ilo. org/public/english/protection/safework/cis/products/ dbs.htm), including some of those listed below.

- ILO Encyclopedia of Occupational Health and Safety: This searchable web version of the encyclopedia includes sections on occupational skin diseases, occupational contact dermatitis, and the prevention of occupational dermatoses. The Encyclopedia can be searched by chemical, industry, and occupation or for potential dermal hazards within each of the previously described categories.

- International Chemical Safety Cards: This searchable database summarizes essential health and safety information on chemicals used by workers and employers in factories, agriculture, construction, and other work places. Information on skin exposure potential is included. This information is available in a wide variety of languages.

- International Risk Phrases Definitions: Risk phrases used by countries in the European Union, including phrases used to classify dermal exposure risks.

- International Hazard Datasheets on Occupations: the International Hazard Datasheets on Occupations are a multipurpose information resource containing information on hazards, risks, and guidelines for prevention related to specific occupations. Dermal hazards associated with listed occupations, when present, are given. 
Article ID:

Citation:

Resource type:

Educational materials:

Number of references:

Industries/occupations:

Specific process:

Chemical:

Specific chemicals:

Mixtures:

Audience:

Topics addressed:

Summary:

\section{3}

International Safety Equipment Association (ISEA)

[2005]. American national standard for hand protection selection criteria. Arlington, VA: ISEA, Report \#ANSI/ISEA 105-2005.

Guideline

No

General-overview

General-overview

No

Professional

F Risk management

F.1 Exposure control strategies

F.1.D PPE and PPE rules

This publication addresses the classification and testing of hand protection for specific performance properties related to chemical and industrial applications. Hand protection includes gloves, mittens, partial gloves, or other items covering the hand or a portion of the hand intended to provide protection against or resistance to a specific hazard. Information can be downloaded from the International Safety Equipment Association's Web site (http://www.safetyequipment.org/glovestd.htm)

Article ID:

114

\section{Citation:}

Resource type:

Educational materials:

Number of references:

Industries/occupations:

Specific process:

Chemical:

Specific chemicals:

Mixtures:
Institute of Medicine (IOM) [2004]. CEFIC workshop on methods to determine dermal permeation for human risk assessment. Riccarton, Edinburgh, UK: IOM, Research Report TM/04/07.

Technical publication/report

No

40

No 
Audience:

Topics addressed:

Summary:
Professional

A Overview

A.4 Skin physiology and function as barriers to chemical insults

C Exposure characterization

C.2 Description of factors influencing exposure conditions

C.2.E Uptake

C.5 Exposure modeling

D Hazard identification

D.3 Characterization protocols

D.3.E Measurement of skin permeation rates and reservoir effects

D.3.F QSAR—development, validation, and application

E Risk assessment

E.2 Example of risk assessments

This workshop's aim was to develop recommendations for methods to determine dermal permeation rates for use in human risk assessments. This was proposed to be done within the context of the possible future regulatory framework for chemical risk assessment (REACH). The main outcomes of the meeting were:

- A definition of a standardized protocol for an in vitro method for measuring dermal absorption of industrial chemicals after infinite and finite doses, to be used to produce data for the development of predictive relationships.

- Recommendations on the existing status and reliability of QSAR data.

- Recommendations on the role of model predictions in generating absorption data for risk assessment.

- Recommendations for a strategy for using measurements and predictions of dermal permeation to meet the requirements of REACH.

- Suggestions on the steps that will be needed to develop this strategy.
Article ID:

Citation:

\section{5}

Kalnas J, Teitelbaum DT [2000]. Dermal absorption of benzene: Implications for work practices and regulations. Int J Occup Environ Health 6(2):114-21. 
Resource type:

Educational materials:

Number of references:

Industries/occupations:

Specific process:

Chemical:

Specific chemicals:

Mixtures:

Audience:

Topics addressed:

Summary:

Article ID:

Citation:

Resource type:

Educational materials:

Number of references:

Industries/occupations:

Specific process:

Chemical:
Journal article—review, meta-analysis

No

49

solvents

benzene

No

Professional

A Overview

A.1 Occurrence of skin exposures in the workplace

A.5 Dermal regulations and skin notations

C Exposure characterization

C.2 Description of factors influencing exposure conditions

C.2.E Uptake

C.5 Exposure modeling

E Risk assessment

E.1 Guidelines for risk assessment or analysis

E.1.B Systemic health effects

E.2 Example of risk assessments

This paper provides an overview of occupational dermal exposure to benzene. Topics discussed include estimates of the amount of benzene absorbed through the skin and the increased likelihood of developing leukemia at low exposure levels, the development of permissible exposure limits for benzene, and proposed exposure limits from the NIOSH and ACGIH.
116

Kampf G, Loffler H [2003]. Dermatological aspects of a successful introduction and continuation of alcohol-based hand rubs for hygienic hand disinfection. J Hosp Infect 55(1).

Journal article—review, meta-analysis

No

61

Service-Medical

cleaning agents, hand cleansers, soaps and detergents 
Specific chemicals:

Mixtures:

Audience:

Topics addressed:

Summary:

Article ID:

Citation:

Resource type:

Educational materials:

Number of references:

Industries/occupations:

Specific process:
No

Professional

D Hazard identification

D.1 Potential health effects resulting from specific chemicals

D.1.A Irritant contact dermatitis

F Risk management

F.1 Exposure control strategies

F.1.E Skin management, barrier creams, moisturizers, cleansers, and rubs

This review of alcohol-based hand rubs in hospitals found that after years of hand washing, 30\% of healthcare workers incur occupational hand dermatitis, mostly contact dermatitis (allergic reactions were rare). Steps to prevent irritant contact dermatitis are also presented.
117

Kanerva L [2000]. Handbook of occupational dermatology. Berlin, NY: Springer.

Book/monograph, whole

No

8205

Beauty/Cosmetology, Construction, Manufacturing-Other

Aircraft Industry Workers, Air Hammer Operators, Aromatherapists, Asphalt Workers (Paving), Automobile Mechanics, Bakers, Barbers and Hairdressers, Bartenders, Bath Attendants, Batik Manufacturing Workers, Battery Makers, Beekeepers, Biotechnical Industry Workers, Boat Builders, Brake-Lining Workers, Butchers and Slaughterhouse Workers, Cabinet Makers, Candle Makers, Confectionery and Candy Makers, Carpenters Car Industry, Cement Workers, Ceramic and Pottery Workers, Cheese Makers, Chemists, Child Daycare Workers, Cigarette and Cigar Makers, Construction Workers, Cosmetologists, Dental Personnel, Detergent Workers, Divers, Electron Microscopy Workers, Electronic Workers, Electroplaters, Embalmers, Engravers, Farmers and Farm Workers, Floor Layers, Florists, Forestry Workers, Foundry Workers, Fur Farming and the Fur Industry, Furniture Manufacture, Gardeners, Glass Workers, Grinders and Brazers of Hard Metal, Hairdressers, Health Care Workers, 
Chemical:

Specific chemicals:

Mixtures:

Audience:

Topics addressed:

Summary:
Highway Construction Workers, Histology Technicians, House Workers, Insulation Workers, Jewelers, Laboratory Technicians, Leather Industry, Locksmiths, Machinists, Masseurs, Mechanics, Metal Industry, Metal Polishers, Military Personnel, Mining (Tunneling) Workers, Musicians, Office Workers, Oil-Rig Workers, OperatingRoom Staff, Painters, Lacquerers and Varnishers, Paper and Pulp Workers, Pharmaceutical and Cosmetic Industries Workers, Photographers and Other Photo-Lab Workers, Poultry Processors, Pitch Workers, Plumbers and Pipe Fitters, Printers and Lithographers, Professional Sports: Skin Disorders in Athletes, Railroad Shop Workers, Reindeer Herders, Roofers, Shoe Manufacturers and Repairers, Silk-Screen Workers, Stonemasons, Sugar Artists, Swimming Pool Workers, Tattoo Artists, Textile Workers, Veterinary Surgeons, Welders, Winemakers

Hand cleansers, heavy metals/inorganic compounds, latex, pesticides, plastics and resins, rubber additives, solvents, other: disinfectants, formaldehyde, pharmaceuticals, fragrances, enzymes, cement, textiles, leather, adhesives, electronics, paints, polymers, cutting fluids, rubber, plants, spices, and woods.

No

Professional

D Hazard identification

D.1 Potential health effects resulting from specific chemicals

D.1.A Irritant contact dermatitis

D.1.B Allergic contact dermatitis/sensitization

D.1.C Systemic toxicity

F Risk management

F.1 Exposure control strategies

F.1.C Work practice/Administrative controls

F.1.D PPE and PPE rules

F.1.E Skin management, barrier creams, moisturizers, cleansers, and rubs

This comprehensive handbook is comprised of dozens of separate articles on occupation skin exposures and is designed to provide information to healthcare professionals for dealing with patients. Diseases covered include: allergic and irritant dermatitis (AID), contact urticaria, photodermatoses, infectious diseases, skin tumors, systematic reactions due to percutaneous 
absorption, predisposed diseases, and occupational skin problems.

The handbook is divided into four sections.

Part 1-Epidemiology, Treatment, and Prognosis (57 articles).

Part 2-Substances and Products (articles on chemical substances) (36 articles)

Part 3-Job Descriptions with their Irritants and Allergens (94 articles)

Part 4-Chemistry and Concentrations of Patch test Allergens (3 articles)

Article ID:

Citation:

Resource type:

Educational materials:

Number of references:

Industries/occupations:

Specific process:

Chemical:

Specific chemicals:

Mixtures:

Audience:

Topics addressed:

\section{8}

Kimber, I [1996]. The role of the skin in the development of chemical respiratory hypersensitivity. Toxicol Lett 86(2-3):89-92.

Journal article—review, meta-analysis

No

29

\section{No}

Professional

A Overview

A.2 Health hazards resulting from skin exposure to chemicals

D Hazard identification

D.1 Potential health effects resulting from specific chemicals

D.1.B Allergic contact dermatitis/sensitization

D.1.C Systemic toxicity

D.1.D Other health effects

This paper examines the mechanisms relevant to the stimulation of respiratory sensitization following cutaneous exposure to chemical allergens and implications for the prevention of occupational asthma. 
Article ID:

Citation:

Resource type:

Educational materials:

Number of references:

Industries/occupations:

Specific process:

Chemical:

Specific chemicals:

Mixtures:

Audience:

Topics addressed:

Summary:

Article ID:

Citation:

Resource type:

Educational materials:

Number of references:

Industries/occupations:

Specific process:

Chemical:

Specific chemicals:
119

Kimber I, Pichowski JS, Betts CJ, Cumberbatch M, Basketter DA, Dearman RJ [2001]. Alternative approaches to the identification and characterization of chemical allergens. Toxicol In Vitro 15(4-5):307-12.

Journal article-review, meta-analysis

No

29

No

Professional

D Hazard identification

D.1 Potential health effects resulting from specific chemicals

D.1.B Allergic contact dermatitis/sensitization

D.3 Characterization protocols

D.3.B Irritation potential

D.3.C Sensitization potential

This paper describes some of the general requirements of in vitro test methods for skin sensitization and progress that has been made in developing suitable approaches with particular emphasis on the utility of dendritic cell culture systems.

\section{0}

Kissel J, Fenske R [2000]. Improved estimation of dermal pesticide dose to agricultural workers upon reentry. Appl Occup Environ Hyg 15(3):284-90.

Journal article_-review, meta-analysis

No

19

Agricultural 
Mixtures:

Audience:

Topics addressed:

Summary:
No

Professional

C Exposure characterization

C.2 Description of factors influencing exposure conditions

C.2.E Uptake

C.5 Exposure modeling

This article presents a method for agricultural worker dermal dose estimation which accounts for the effect of delay in post-shift washing on dose.

Article ID:

\section{1}

Citation:

Resource type:

Educational materials:

Number of references:

Industries/occupations:

Specific process:

Chemical:

Specific chemicals:

Mixtures:

Audience:

Topics addressed:

Summary:
Kissel JC, Richter KY, Fenske RA [1996]. Factors affecting soil adherence to skin in hand-press trials. Bull Environ Contam Toxicol 56(5):722-28.

Journal article—review, meta-analysis

No

12

No

Professional

C Exposure characterization

C.2 Description of factors influencing exposure conditions

C.2.C Skin area affected

This paper compares three approaches for estimating soil adherence to the skin for use in dermal risk estimates (listed below). Each approach offers information of value. Laboratory studies provide an opportunity for systematic examination of the possible effects of soil characteristics on adherence.

1. Laboratory studies using artificial loading scenarios (Que Hee, 1985; Driver, 1989; Sheppard and Evenden, 1992).

2. $\mathrm{Pb}$ exposure studies reporting $\mathrm{Pb}$ concentrations in soil and dust (Roels, 1980; Charney, 1980; Gallacher, 1984; Duggan, 1985). 
3. Direct field measurement using gravimetric methods (Lepow, 1975).

Article ID:

\section{Citation:}

Resource type:

Educational materials:

Number of references:

Industries/occupations:

Specific process:

Chemical:

Specific chemicals:

Mixtures:

Audience:

Topics addressed:

Summary:

\section{2}

Klingner TD, Boeniger [2002] MF. A critique of assumptions about selecting chemical-resistant gloves: A case for workplace evaluation of glove efficacy. Appl Occup Environ Hyg 17(5):360-67.

Journal article—review, meta-analysis

No

48

Yes

Professional

A Overview

A.5 Dermal regulations and skin notations

B Surveillance and clinical aspects

B.1 Surveillance study reporting incidences of occupational skin exposures

B.1.A Skin exposure major focus

D Hazard identification

D.1 Potential health effects resulting from specific chemicals

D.1.A Irritant contact dermatitis

D.1.B Allergic contact dermatitis/sensitization

D.1.C Systemic toxicity

F Risk management

F.1 Exposure control strategies

F.1.D PPE and PPE rules

Those selecting gloves should not rely only upon the manufacturers' laboratory-generated chemical permeation data, for this data may not reflect conditions in the actual workplace (e.g., elevated temperature, flexing, pressure, and product variation between suppliers). This article presents glove selection criteria and recommends dermal monitoring to evaluate glove performance under actual use conditions. 
Article ID:

Citation:

Resource type:

Educational materials:

Number of references:

Industries/occupations:

Specific process:

Chemical:

Specific chemicals:

Mixtures:

Audience:

Topics addressed:

\section{3}

Klotz A, Veeger M, Rocher W [2003]. Skin cleansers for occupational use: testing the skin compatibility of different formulations. Int Arch Occup Environ Health 76(5):367.

Journal article—primary

No

17

Abrasives, hand cleansers, solvents

No

Professional

A Overview

A.4 Skin physiology and function as barriers to chemical insults

F Risk management

F.1 Exposure control strategies

F.1.E Skin management, barrier creams, moisturizers, cleansers, and rubs

This article presents an overview of skin-cleansing products and their ingredients and discusses skin compatibility and cleansing effectiveness. The authors advocate a range of skin cleansers depending upon the degree of contamination. They also recommend avoiding solvents and abrasives to prevent occupational dermatitis, and stress the importance of worker education.

Article ID:

\section{4}

Citation:

Resource type:

Educational materials:

Number of references:

Industries/occupations:

Specific process:
Koch P [2001]. Occupational contact dermatitis: recognition and management. Am J Clin Dermatol 2(6):353-65.

Journal article—review, meta-analysis

No

84

Beauty/Cosmetology, Construction, ManufacturingOther, Service-Food, Service-Medical

Hairdressers, dental laboratory technicians, healthcare workers, metal workers, leather and shoe workers, bakers, caterers, confectioners, and cooks 
Chemical:

Specific chemicals:

Mixtures:

Audience:

Topics addressed:

Summary:
Latex, organic dyes, pesticides, rubber additives, solvents, other: concrete, glues, leather

Use, concentration, associated industry, and health effects are presented for dozens of chemicals.

No

Professional
A Overview
A.2 Health hazards resulting from skin exposure to chemicals
C Exposure characterization
C.2 Description of factors influencing exposure conditions
C.2.B Exposure concentration
D Hazard identification
D.1 Potential health effects resulting from specific chemicals
D.1.A Irritant contact dermatitis

This paper primarily focuses on eight broad occupational categories at risk for occupational contact dermatitis (OCD) and dozens of their associated chemical irritants and sensitizers. The categories are hairdressers, dental laboratory technicians, healthcare workers, construction industry workers, metal workers, leather and shoe workers, florists and gardeners, and food service workers (bakers, caterers, confectioners, and cooks).

125

Article ID:

Kresken J, Klotz A [2003]. Occupational skin-protection products-a review. Int Arch Occup Environ Health 76(5):355-58.

Resource type:

Journal article—review, meta-analysis

Educational materials:

No

Number of references:

34

Industries/occupations:

Specific process:

Chemical:

Hand cleansers, water

Specific chemicals:

Mixtures:

No

Audience:

Topics addressed:

\section{Professional}

D Hazard identification 
D.1 Potential health effects resulting from specific chemicals

D.1.A Irritant contact dermatitis

D.1.B Allergic contact dermatitis/sensitization

F Risk management

F.1 Exposure control strategies

F.1.E Skin management, barrier creams, moisturizers, cleansers, and rubs

Summary:

This paper evaluates the use of skin protection products, including barrier creams, in preventing occupational dermal exposures. They conclude barrier creams do not replace PPE and should only be used against low-grade irritants such as water, detergents, and cutting fluids.

Article ID:

\section{6}

Citation:

Resource type:

Educational materials:

Number of references:

Industries/occupations:

Specific process:

Chemical:

Specific chemicals:

Mixtures:

Audience:

Topics addressed:
Kromhout H, Vermeulen R [2001]. Temporal, personal and spatial variability in dermal exposure. Ann Occup Hyg 45(4):257-73.

Journal article-review, meta-analysis

No

28

Agricultural, Construction, Manufacturing

Rubber manufacturing, Asphalt paving, Coke production

Pesticides, PAHs, paint

Yes

Professional

C Exposure characterization

C.1 Workplace factors associated with harmful skin exposures

C.2 Description of factors influencing exposure conditions

C.2.A Exposure intensity/frequency/duration

C.2.C Skin area affected

C.5 Exposure modeling

E Risk assessment

E.2 Example of risk assessments

A database of dermal exposure measurements (DERMDAT) comprising data from 20 surveys was created 
from agricultural and industrial workers containing 6,400 observations. Analyses of variability showed median values of the total, within-, and between-worker geometric standard deviations to be similar to that published previously for respiratory exposure.

Article ID:

127

Citation:

Resource type:

Educational materials:

Number of references:

Industries/occupations:

Specific process:

Chemical:

Specific chemicals:

Mixtures:

Audience:

Topics addressed:

Summary:
Kutting B, Drexler H [2003]. Effectiveness of skin protection creams as a preventive measure in occupational dermatitis: a critical update according to criteria of evidence-based medicine. Int Arch Occup Environ Health 76(4):253-59.

Journal article—review, meta-analysis

No

63

No

Professional

D Hazard identification

D.1 Potential health effects resulting from specific chemicals

D.1.A Irritant contact dermatitis

F Risk management

F.1 Exposure control strategies

F.1.E Skin management, barrier creams, moisturizers, cleansers, and rubs

This paper reviews the literature to answer the questions: (1) Can a skincare regimen effectively reduce or eliminate work-related poor skin conditions? (2) Do protective creams prevent harmful substances from penetrating and adhering to the skin? (3) Is the differentiation between preexposure and postexposure products justified by reliable data? The authors also address the merit of the traditional three-step skin protection program: skin protection before work, cleaning, and skin care after work. This paper concludes that not enough data have been accumulated to prove the benefit of skin protection measures under real workplace conditions. 
Article ID:

Citation:

Resource type:

Educational materials:

Number of references:

Industries/occupations:

Specific process:

Chemical:

Specific chemicals:

Mixtures:

Audience:

Topics addressed:
128

Leggat PA, Kedjarune U [2003]. Toxicity of methyl methacrylate in dentistry. Int Dent J 53(3):126-31.

Journal article—review, meta-analysis

No

50

Service-Medical, Dentistry

Plastics and resins

Methyl methacrylate (MMA)

No

Professional

B Surveillance and clinical aspects

B.1 Surveillance study reporting incidences of occupational skin exposures

B.1.B Skin exposure minor focus

D Hazard identification

D.1 Potential health effects resulting from specific chemicals

D.1.C Systemic toxicity

F Risk management

F.1 Exposure control strategies

F.1.B Engineering controls

F.1.C Work practice/Administrative controls

F.1.D PPE and PPE rules

Summary:

This paper presents health effects associated with exposure to MMA in dentistry. It includes a discussion of control strategies to use to reduce exposure to MMA.

Article ID:

\section{9}

Citation:

Finnish Institute of Occupational Health (FIOH) [2001]. Epidemiology of skin and respiratory diseases among hairdressers. Helsinki, Finland: FIOH

Resource type:

Technical publication/report

Educational materials:

No

Number of references: $\quad 298$

Industries/occupations:

Beauty/Cosmetology

Specific process:

Hairdressers, salons 
Chemical:

Specific chemicals:

Mixtures:

Audience:

Topics addressed:

Summary:
Organic dyes, soaps and detergents

Ammonium persulfate

No

Professional

A Overview

A.1 Occurrence of skin exposures in the workplace

A.2 Health hazards resulting from skin exposure to chemicals

B Surveillance and clinical aspects

B.1 Surveillance study reporting incidences of occupational skin exposures

B.1.A Skin exposure major focus

B.3 Surveillance study protocols/procedures for gathering data

C Exposure characterization

C.1 Workplace factors associated with harmful skin exposures

C.4 Direct methods to measure exposure

C.4.B Skin

D Hazard identification

D.1 Potential health effects resulting from specific chemicals

D.1.A Irritant contact dermatitis

D.1.B Allergic contact dermatitis/sensitization

D.1.D Other health effects

F Risk management

F.1 Exposure control strategies

F.1.B Engineering controls

This paper includes the following: Section 1 presents introductory material on dermal and respiratory exposure among hairdressers (15 pages). Section 2 presents findings of a literature review (27 pages). Section 3 to 7 present methods, results, discussion, and conclusions regarding 20 Finnish salons (50 pages). Section 8 is the reference list (32 pages).

In addition to an extensive literature review, this paper presents five epidemiologic studies of skin and respiratory disorders among hairdressers. Study I focuses on the working conditions in salons and the perceived health of the hairdressers. Studies II, III, and IV focus on the prevalence, incidence, and risk of skin and respiratory symptoms and diseases among hairdressers. Study 
$\mathrm{V}$ focuses on the risks of and causes for leaving the profession. Environmental data were collected at 20 Finnish salons. Health data were collected by questionnaire, phone interviews, and medical examinations. The hairdressing salons meet Finnish indoor air criteria, but high peak concentrations of certain chemicals, including ammonium persulfate, were found to cause skin and respiratory diseases. Hairdressers incur an increased incidence of asthma and chronic bronchitis. Local exhaust ventilation was recommended.

Ammonium persulfate is used as a polymerization initiator in polymer chemistry, as an etchant and cleaner in manufacture of printed circuit boards, as a booster in hair bleaching formulations in cosmetics, and as a secondary oil recovery system by acting as a polymerization initiator and a gel breaker.

Article ID:

\section{Citation:}

Resource type:

Educational materials:

Number of references:

Industries/occupations:

Specific process:

\section{Chemical:}

Specific chemicals:

Mixtures:

Audience:

Topics addressed:
130

Leung HW, Paustenbach DJ [1994]. Techniques for estimating the percutaneous absorption of chemicals due to occupational and environmental exposure. Appl Occup Environ Hyg 9(3):187-97.

Journal article—review, meta-analysis

No

92

No

Professional

C Exposure characterization

C.2 Description of factors influencing exposure conditions

C.2.A Exposure intensity/frequency/duration

C.2.B Exposure concentration

C.2.C Skin area affected

C.2.E Uptake

C.4 Direct methods to measure exposure

C.4.A Surfaces

C.5 Exposure modeling 
Summary:

Article ID:

Citation:

Resource type:

Educational materials:

Number of references:

Industries/occupations:

Specific process:

Chemical:

Specific chemicals:

Mixtures:

Audience:

Topics addressed:
D Hazard identification

D.3 Characterization protocols

D.3.E Measurement of skin permeation rates and reservoir effects

This paper reviewed techniques for estimating the percutaneous absorption of chemicals following occupational exposure. It discusses factors influencing percutaneous absorption including number of exposures, nature of broken skin, exposure site, chemical uptake, and skin surface area. The latter was considered to be the most important factor. Discusses absorption studies, modeling, calculating exposure, and the interpretation of wipe sample data.

131

Liu Y, Bello D, Sparer JA, Stowe MH, Gore RJ, Woskie SR, Cullen MR, Redlich CA [2007]. Skin exposure to aliphatic polyisocyanates in the auto body repair and refinishing industry: A qualitative assessment. Ann Occup Hyg 51(5):429-39.

Journal article-primary

No

22

Manufacturing-Automotive auto body repair, refinishing Autobody workers

Paint

Aliphatic polyisocyanate, hexamethylene diisocyanate (HDI), isophorone diisocyanate (IPDI)

No

Professional

A Overview

A.1 Occurrence of skin exposures in the workplace

B Surveillance and clinical aspects

B.1 Surveillance study reporting incidences of occupational skin exposures

B.1.A Skin exposure major focus

C Exposure characterization

C.2 Description of factors influencing exposure conditions

C.2.B Exposure concentration

C.2.C Skin area affected 


$\begin{array}{ll}\text { C.2.E } & \text { Uptake } \\ \text { C.4 } & \text { Direct methods to measure exposure } \\ \text { C.4.A } & \text { Surfaces } \\ \text { C.4.B } & \text { Skin } \\ \text { D } & \text { Hazard identification } \\ \text { D.3 } & \text { Characterization protocols } \\ \text { D.3.E } & \text { Measurement of skin permeation rates and } \\ & \text { reservoir effects } \\ \text { D.4 } & \text { Other } \\ \text { E } & \text { Risk assessment } \\ \text { E.3 } & \text { Other } \\ \text { F } & \text { Risk management } \\ \text { F.1 } & \text { Exposure control strategies } \\ \text { F.1.D } & \text { PPE and PPE rules }\end{array}$

Summary:

This study evaluated aliphatic isocyanate skin exposure among auto body shop workers. Also evaluated was the effectiveness of gloves and other PPE.

Article ID:

132

Citation:

Resource type:

Educational materials:

Number of references:

Industries/occupations:

Specific process:

\section{Chemical:}

Specific chemicals:

Mixtures:

Audience:

Topics addressed:
Lowney YW, Ruby MV, Wester RC, Schoof RA, Holm S E, Hui XY, Barbadillo S, Maibach HI [2005]. Percutaneous absorption of arsenic from environmental media. Toxicol Ind Health 21(1-2):1-14.

Journal article-review, meta-analysis

No

30

Arsenic

No

Professional

C Exposure characterization

C.2 Description of factors influencing exposure conditions

C.2.B Exposure concentration

C.2.E Uptake 
Summary:

Article ID:

\section{Citation:}

Resource type:

Educational materials:

Number of references:

Industries/occupations:

Specific process:

Chemical:

Specific chemicals:

Mixtures:

Audience:

Topics addressed:

Summary:
This paper addresses what is known about percutaneous absorption of arsenic based on studies of rhesus monkeys and offers study design considerations including particle size, application rates, means of ensuring skin contact, and appropriate statistical evaluation of the data. The authors conclude that there are likely to be many site- or samplespecific factors that control the absorption of arsenic, and matrix-specific analyses may be required to understand the degree of percutaneous absorption.
133

Lushniak BD [1995]. The epidemiology of occupational contact dermatitis. Dermatol Clin 13(3):671-80.

Journal article—review, meta-analysis

No

66

Agricultural, Construction, Forestry/Fisheries, Manufacturing-Chemical, Manufacturing-Other, Mining, Service—Food, Service-Medical, ServiceTransportation/Communications/Utility, Trade, finance/ insurance/realty, meat products, leather, motorvehicles
No

Professional

B Surveillance and clinical aspects

B.1 Surveillance study reporting incidences of occupational skin exposures

B.1.A Skin exposure major focus

B.2 Loss of workdays and impact on productivity

B.3 Surveillance study protocols/procedures for gathering data

D Hazard identification

D.1 Potential health effects resulting from specific chemicals

D.1.A Irritant contact dermatitis

This article reviews occupational contact dermatitis epidemiologic data sources for important information on prevalence, diagnosis, public health importance, risk factors, etiologic agents, prognosis, and preventive 
measures. It also provides incidences for different occupational groups.

Article ID:

Citation:

Resource type:

Educational materials:

Number of references:

Industries/occupations:

Specific process:

Chemical:

Specific chemicals:

Mixtures:

Audience:

Topics addressed:

\section{4}

Lushniak BD [2003]. The importance of occupational skin diseases in the United States. Int Arch Occup Environ Health 76(5):325-30.

Journal article—review, meta-analysis

No

22

General-overview

Provides data by major occupational categories
No

Professional

A Overview

A.1 Occurrence of skin exposures in the workplace

B Surveillance and clinical aspects

B.1 Surveillance study reporting incidences of occupational skin exposures

B.1.A Skin exposure major focus

B.2 Loss of workdays and impact on productivity

This epidemiological study presents occupational skin disease and disorder surveillance data for the U.S. and three states $(\mathrm{OH}, \mathrm{OR}$, and WA). It describes trends, data by occupation, lost time, and other data.

Article ID:

\section{5}

Citation:

Resource type:

Educational materials:

Number of references:

Industries/occupations:

Specific process:
Lushniak BD [2004]. Occupational contact dermatitis. Dermatol Ther 17(3):272-77.

Journal article-review, meta-analysis

No

32

General-overview, Agricultural, Construction, Forestry/ Fisheries, Manufacturing_Chemical, Mining, ServiceMedical, Transportation/Communications/Utility 


\section{Chemical:}

Specific chemicals:

Mixtures: $\quad$ No

Audience:

Professional

Topics addressed:

A Overview

A.1 Occurrence of skin exposures in the workplace

A.2 Health hazards resulting from skin exposure to chemicals

A.3 Investigation, intervention, and control of occupational skin exposures

B Surveillance and clinical aspects

B.1 Surveillance study reporting incidences of occupational skin exposures

B.1.A Skin exposure major focus

B.2 Loss of workdays and impact on productivity

C Exposure characterization

C.1 Workplace factors associated with harmful skin exposures

D Hazard identification

D.1 Potential health effects resulting from specific chemicals

D.1.A Irritant contact dermatitis

D.1.B Allergic contact dermatitis/sensitization

D.1.D Other health effects

F Risk management

F.1 Exposure control strategies

F.1.A Substitution

F.1.D PPE and PPE rules

Summary:

This paper presents an overview of issues involved in the study of occupational contact dermatitis, including importance, incidence, economic impact, at-risk occupations, diagnosis, and prevention.

Article ID: 136

Citation:

Mansdorf SZ, Henry N III [2003]. Personal protective clothing. In: DiNardi SR, ed. The occupational environment: Its evaluation, control, and management, 2nd ed. Fairfax, VA: American Industrial Hygiene Association.

Resource type:

Book/monograph, chapter 
Educational materials:

Number of references:

Industries/occupations:

Specific process:

Chemical:

Specific chemicals:

Mixtures:

Audience:

Topics addressed:

Summary:
No

42

No

Professional

F Risk management

F.1 Exposure control strategies

F.1.D PPE and PPE rules

Chapter 35, "Personal Protective Clothing," from the AIHA book, The Occupational Environmental: Its Evaluation Control and Management (the White Book), discusses PPE for chemical hazards as well as thermal, mechanical, radiological, and biological hazards. It discusses performance characteristics, ergonomics, cost, maintenance, and training for different types of personal protective equipment used to control dermal hazards.

\section{7}

Marks JG [2002]. Contact and occupational dermatology, 3rd ed. St. Louis: Mosby.

Resource type:

Book/monograph, whole

Educational materials:

No

Number of references:

Industries/occupations:

General—overview, Agricultural, Beauty/Cosmetology, Cleaning/Janitorial/Maid, Construction, ManufacturingOther, Medical Services

Specific process:

Electronics workers, Dental workers, Florists, Food service workers, Machinists, Office workers, Photographers, Printers, Textile

Chemical:

Food products

Specific chemicals:

Preservatives

Mixtures:

No

Audience:

Topics addressed:
Professional

B Surveillance and clinical aspects

B.4 Clinical protocols for recognition of skin exposure health effects

C Exposure characterization 
Summary:

Article ID:

Citation:

Resource type:

Educational materials:

Number of references:

Industries/occupations:

Specific process:
C.4 Direct methods to measure exposure

C.4.B Skin

D Hazard identification

D.1 Potential health effects resulting from specific chemicals

D.1.A Irritant contact dermatitis

D.1.B Allergic contact dermatitis/sensitization

D.3 Characterization protocols

D.3.C Sensitization potential

This book covers the diagnosis and management of suspected contact and occupational dermatitis. The early chapters of this book focus on the nonoccupational aspects of dermatology but include discussions of dermatitis recognition, monitoring, and treatment, as well as health effects from preservatives, vehicles, cosmetics, fragrances, and hair and nail care. The focus is on treatment rather than prevention.

Chapters 12 through 17 cover occupational dermatology: These chapters are:

Ch. 12 Etiology of Occupational Skin Disease Workers

Ch. 13 Evaluation of the Worker in the Office and at the Work Site

Ch. 14 Management of Occupational Dermatitis

Ch. 15 Occupations Commonly Associated With Contact Dermatitis

Ch. 16 Contact Urticaria

Ch. 17 Contact Dermatitis in Children

\section{8}

Marquart H, Maidment S, McClaflin JL, Fehrenbacher MC. Harmonization of future needs for dermal exposure assessment and modeling: A workshop report. Appl Occup Environ Hyg 16(2):218-27.

Journal article_-review, meta-analysis

No

42 


\section{Chemical:}

Specific chemicals:

Mixtures:

No

Audience:

Professional

Topics addressed:

A Overview

A.5 Dermal regulations and skin notations

C Exposure characterization

C.2 Description of factors influencing exposure conditions

C.2.E Uptake

C.4 Direct methods to measure exposure

C.4.A Surfaces

C.4.B Skin

Summary:

\section{C.5 Exposure modeling}

This article is a summary of the 1999 International Symposium on Occupational Exposure Databases and Their Application for the Next Millennium held in London. The workshop was organized in an effort to harmonize future needs in this area. It discusses what is known about methods to measure the amount of contaminant on the skin and surfaces, the amount of contaminant absorbed through the skin, and merits of these approaches. It also discusses what is needed in the field, including raising awareness among occupational health practitioners and creating simple tools for smalland medium-sized businesses to use in risk assessment and management activities.

Article ID: 139

Citation:

Resource type:

Educational materials:

Number of references:

Industries/occupations:

Specific process:

Chemical:

Specific chemicals:

Mixtures:
Marquart J, Brouwer DH, Gijsbers JH, Links IH, Warren $\mathrm{N}$, van Hemmen JJ [2003]. Determinants of dermal exposure relevant for exposure modeling in regulatory risk assessment. Ann Occup Hyg 47(8):599-607.

Journal article—review, meta-analysis

No

71

No 
Audience:

Topics addressed:

Summary:

Article ID:

Citation:

Resource type:

Educational materials:

Number of references:

Industries/occupations:

Specific process:
Professional

C Exposure characterization

C.1 Workplace factors associated with harmful skin exposures

C.2 Description of factors influencing exposure conditions

C.2.A Exposure intensity/frequency/duration

C.5 Exposure modeling

E Risk assessment

E.1 Guidelines for risk assessment or analysis

E.1.A Localized health effects

E.1.B Systemic health effects

This article is the 3rd article of a six-part series on RISKOFDERM, a tool for conducting risk assessments. The series was published in the Annals of Occupational Hygiene in 2003. The following briefly summarizes each paper in the series:

1. ID 212-Outlines a "toolkit" for conducting dermal occupational risk assessment.

2. ID 163-Describes the assumptions in the toolkit and describes an approach to exposure assessment used by the toolkit.

3. ID 139-Describes the determinants relevant for dermal exposure models in the scope of regulatory risk assessment.

4. ID 219-Describes how default dermal exposure values can be adjusted for specific work situations.

5. ID 100-Describes the derivation of the toolkit's default task-based dermal exposure values.

6. ID 193-Describes the development of "intrinsic toxicity" (IT) scores used for hazard characterization.
Mathur AK, Khanna SK [2002]. Dermal toxicity due to industrial chemicals. Skin Pharmacol Appl Skin Physiol 15(3):147-53.

Journal article—review, meta-analysis

No

21 
Chemical:

Specific chemicals:

Mixtures:

Audience:

Topics addressed:
Summary:

Article ID:

Citation:

Resource type:

Educational materials:

Number of references:

Industries/occupations:

Specific process:

Chemical:

Specific chemicals:

Mixtures:

Audience:
Cleaning agents, coolants, heavy metals/inorganic compounds, latex, organic dyes, plastics and resins, rubber additives, soaps and detergents, solvents, other: florescent whitening agents, dyes, adhesives, perfume, preservatives

Dozens of specific chemicals are addressed

No

Professional

A Overview

A.4 Skin physiology and functions as a barrier to chemical insults

D Hazard identification

D.1 Potential health effects resulting from specific chemicals

D.1.A Irritant contact dermatitis

D.1.B Allergic contact dermatitis/sensitization

D.1.C Systemic toxicity

D.1.D Other health effects

F Risk management

F.1 Exposure control strategies

F.1.E Skin management, barrier creams, moisturizers, cleansers, and rubs

The paper discusses potential health effects from exposure to metals, florescent whiting agents, dyes, adhesives and resins, preservatives and disinfectants, plastics and rubbers, perfume, soaps and detergents, and cutting oils and solvents.

\section{1}

McArthur B [1992]. Dermal measurement and wipe sampling methods: A review. Appl Occup Environ Hyg 7(9):599-606.

Journal article—review, meta-analysis

No

81

No

Professional 
Topics addressed:

Summary: $\begin{array}{ll}\text { C } & \text { Exposure characterization } \\ \text { C.4 } & \text { Direct methods to measure exposure } \\ \text { C.4.A } & \text { Surfaces } \\ \text { C.4.B } & \text { Skin } \\ \text { C.4.C } & \text { Biomonitoring }\end{array}$

This article discusses various methods for directly measuring dermal exposures to hazardous materials on the skin or clothing and on work surfaces, deposited by patches, skin swabs, rinses, and radioactive or fluorescent tracers. This article also discusses biological monitoring (measuring biomarkers for blood, urine, or exhaled air).
Article ID:

Citation:

\section{Resource type:}

Educational materials:

Number of references:

Industries/occupations:

Specific process:

Chemical:

Specific chemicals:

Mixtures:

Audience:

Topics addressed:

\section{2}

McClean MD, Rinehart RD, Sapkota A, Cavallari JM, Herrick RF [2007]. Dermal exposure and urinary 1-hydroxypyrene among asphalt roofing workers. J Occup Environ Hyg 4(1). (2007): 118-26

Journal article_-primary

No

23

Construction

Asphalt roofing workers

Petroleum products \& lubricants, other: polycyclic aromatic compounds (PACs), coal tar pitch

Pyrene, benzoapyrene (BAP)

No

Professional

A Overview

A.1 Occurrence of skin exposures in the workplace

C Exposure characterization

C.1 Workplace factors associated with harmful skin exposures

C.2 Description of factors influencing exposure conditions

C.2.A Exposure intensity/frequency/duration

C.2.B Exposure concentration

C.2.E Uptake

C.4 Direct methods to measure exposure

C.4.B Skin 
D Hazard identification

D.3 Characterization protocols

D.3.E Measurement of skin permeation rates and reservoir effects

Summary:

This study ascertained determinants of dermal exposure to polycyclic aromatic compounds (PACs) among asphalt roofing workers using dermal patches and urine samples to evaluate the effect of dermal exposure on total absorbed dose. Specific tasks related to roofing included tearing off old roofs, putting down new roofs, and operating the kettle. Results were presented. Dermal exposure was a significant determinant of total absorbed dose.

Article ID:

143

Citation:

Resource type:

Educational materials:

Number of references:

Industries/occupations:

Specific process:

Chemical:

Specific chemicals:

Mixtures:

Audience:

Topics addressed:

Summary:
McDougal JN, Robinson PJ [2002]. Assessment of dermal absorption and penetration of components of a fuel mixture. Sci Total Environ 288(1-2):23-30.

Journal article-review, meta-analysis

No

20

Petroleum products \& lubricants

JP-8 jet fuel, undecane, dodecane, decane, tridecane, tetradecane, methyl naphthalenes, trimethyl benzene, nonane, pentadecane, dimethyl naphthalene, dimethyl benzene (xylene), naphthalene, ethyl benzene, methyl benzene (toluene)

Yes

Professional

C Exposure characterization

C.2 Description of factors influencing exposure conditions

C.2.E Uptake

E Risk assessment

E.2 Example of risk assessments

This article discusses methods for assessing the risks from dermal exposures to complex mixtures, specifically JP-8 jet fuel—a volatile mixture which varies radically in composition depending on the phase of the mixture (vapor, liquid, or aerosol). This article assesses absorption (into the skin) and penetration (through the skin) of 
components in the mixture and discusses why absorption and penetration can differ. Permeability coefficients for 12 components in JP-8 jet fuel were calculated. The authors suggest that absorption and penetration methodologies similar to those used for JP-8 jet fuel could be used to estimate systemic toxicity of other mixtures.

Article ID:

144

Citation:

Resource type:

Educational materials:

Number of references:

Industries/occupations:

Specific process:

Chemical:

Specific chemicals:

Mixtures:

Audience:

Topics addressed:

Summary:
McDougal JN, Boeniger MF [2002]. Methods for assessing risks of dermal exposures in the workplace. Crit Rev Toxicol 32(4):291-327.

Journal article—review, meta-analysis

No

70

No

Professional

A Overview

A.3 Investigation, intervention, and control of occupational skin exposures

A.4 Skin physiology and function as barriers to chemical insults

C Exposure characterization

C.2 Description of factors influencing exposure conditions

C.2.E Uptake

C.5 Exposure modeling

This paper provides a comprehensive and comparative analysis of methods used to estimate both the amount of a chemical contacting the skin (external dose) and the amount that reaches internal organs (internal dose). This paper addresses each step in the process, describes the assumptions involved, assesses the model's strengths and weaknesses, and provides recommendations for further research. The paper discusses the following:

- Internal dose assessment

— Flux and permeability theory 
- Calculations based on empirical measurements and fraction absorbed

- Calculations based on steady-state flux

- Calculations adjusted for square root of time

- Calculations based on biologically based models

- Comparisons with short-term skin penetration data

- Route-to-route extrapolations

- Extrapolation factor approach

- Biologically based models

- Dermal exposure levels

- Skin notation

- Banding approach to dermal exposure risks

- Dermal occupational exposure levels

- Skin absorption time

- Risk characterizations

Article ID:

\section{Citation:}

Resource type:

Educational materials:

Number of references:

Industries/occupations:

Specific process:

Chemical:

Specific chemicals:

Mixtures:

Audience:

Topics addressed:

Summary:

\section{5}

McDougal JN, Council EA III, Powers BS [2007]. Systemic toxicity from skin exposures (or what happens when you do not decontaminate). J Chem Health Saf 14(4):23-31.

Journal article—review, meta-analysis

No

92

Corrosives, heavy metals/inorganic compounds, pesticides, solvents, other: pharmaceuticals

No

General

A Overview

E Risk management

E.4 Guidelines/recommendations for postexposure skin decontamination

This resource provides a general overview of the use and misuse of decontamination after dermal exposure. The author points out that some decontamination procedures can make penetration of a chemical through 
the skin worse. This review evaluates the necessity for decontamination of various chemical classes in the workplace.

Article ID:

Citation:

Resource type:

Educational materials:

Number of references:

Industries/occupations:

Specific process:

Chemical:

Specific chemicals:

Mixtures:

Audience:

Topics addressed:

Summary:
146

Meding B [2000]. Differences between the sexes with regard to work-related skin disease. Contact Dermatitis 43(2):65-71.

Journal article—review, meta-analysis

No

41

Beauty/Cosmetology, Cleaning/Janitorial/Maid, ServiceFood, Service-Medical

Shows high-risk occupations by major group in 1990

Heavy metals/inorganic compounds

Nickel

No

Professional

B Surveillance and clinical aspects

B.1 Surveillance study reporting incidences of occupational skin exposures

B.1.A Skin exposure major focus

D Hazard identification

D.1 Potential health effects resulting from specific chemicals

D.1.A Irritant contact dermatitis

D.1.B Allergic contact dermatitis/sensitization

F Risk management

F.1 Exposure control strategies

F.1.C Work practice/Administrative controls

F.1.D PPE and PPE rules

This paper reviews gender differences in work-related skin disease. Women report skin disease more often than men. They are more often affected than men, and they work in female-dominated occupations (e.g., hairdressing, catering, cleaning, and health-care work) which are more likely to involve wet work. For these occupations, work-related skin disease is common and usually presents as hand eczema, typically, irritant contact dermatitis. Nickel allergy is the most common contact allergy. Control strategy discussion includes a focus on reducing wet exposure. 
Article ID: $\quad 147$

Citation:

Resource type:

Educational materials:

Number of references:

Industries/occupations:

Specific process:

Chemical:

latex

Specific chemicals:

Mixtures:

No

Audience:

Topics addressed:

Summary:

Article ID:

\section{8}

Citation:

Moss GP, Dearden JC, Patel H, Cronin MT [2002].

Quantitative structure-permeability relationships (QSPRs) for percutaneous absorption. Toxicol In Vitro 16(3):299-317.

Resource type:

Educational materials:

Journal article-review, meta-analysis

Number of references:

No

Industries/occupations:

Specific process:

Chemical:

Specific chemicals:

Mixtures:

No

Audience:
Mellstrom GA, Wrangsjo K, Wahlberg JE, Fryklund B

1996]. The value and limitations of protective gloves in medical health service: part III. Dermatol Nurs $8(5): 345-55$.

ournal article_review, meta-analysis

No

Service-Medical

General

A Overview

A.2 Health hazards resulting from skin exposure to chemicals

E Risk management

E.3 "Best practices"/guidelines/recommendations

E.3.D PPE and PPE rules

This paper presents the benefits and problems associated with glove protection from dermal exposures. Describes problems of permeability and side effects (latex allergy).
Professional 
Topics addressed:

Summary:
C Exposure characterization
C.2 Description of factors influencing exposure conditions
C.2.E Uptake
D Hazard identification
D.3 Characterization protocols
D.3.E Measurement of skin permeation rates and reservoir effects
D.3.F QSARs—development, validation, and application

This article reviews the use and validity of the current state-of-the-art in quantitative structure property relationships (QSPRs) and, more specifically, quantitative structure activity relationship (QSARs) used in modeling the absorption of chemicals through the skin.
Article ID:

Citation:

Resource type:

Educational materials:

Number of references:

Industries/occupations:

Specific process:

Chemical:

Specific chemicals:

Mixtures:

Audience:

Topics addressed:
149

Nash JL [2000]. Skin care: starting from scratch. Occupational Hazards 62(4):53-55.

Magazine article

Yes

0

Other

No

General

A Overview

A.1 Occurrence of skin exposures in the workplace

E Risk management

E.1 Overview of skin exposure control options

E.3 "Best practices"/guidelines/recommendations

E.3.A Substitution

E.3.B Engineering controls

E.3.C Work practice/administration controls

E.3.D PPE and PPE rules

E.3.E Skin management, barrier creams, moisturizers, cleansers, and rubs 
Summary:

Article ID:

Citation:

Resource type:

Educational materials:

Number of references:

Industries/occupations:

Specific process:

Chemical:

Specific chemicals:

Mixtures:

Audience:

Topics addressed:

Summary:
This paper provides an overview of occupational skin disease, its underreporting, and prevention.

150

NIOSH [2005]. Recommendations for CPC: A companion to the NIOSH pocket guide to chemical hazards.

[www.cdc.gov/niosh/ncpc/ncpcl.html].

Web page

No

General-overview

Abrasives, cleaning agents, coolants, corrosives, fiberglass and other fibers, heavy metals/inorganic compounds, organic dyes, particulates, pesticides, petroleum products \& lubricants, plastics and resins, solvents

Includes all chemicals in the NIOSH Pocket Guide to Chemical Hazards (ID 152)

No

General

E Risk management

E.3 "Best practices"/guidelines/recommendations

E.3.D PPE and PPE rules

This Web page provides CPC recommendations for all chemicals listed in the NIOSH Pocket Guide to Chemical Hazards, June 1997 Edition (NIOSH Publication No. 97-140). These recommendations are based on another published work, Quick Selection Guide to Chemical Protective Clothing, Third Edition, by Krister Forsberg and S.Z. Mansdorf (1997).

The Pocket Guide provides general recommendations in table format for skin protection according to the following designations:

- Prevent skin contact, meaning that there is a dermal hazard potential.

- Frostbite, meaning there is the potential for freezing of the skin from direct contact with the liquified gas through rapid evaporation.

- N.R. means that no recommendation can be made either because the chemical is not a demonstrated dermal hazard or inadequate information is available. 
Article ID:

Citation:

Resource type:

Educational materials:

Number of references:

Industries/occupations:

Specific process:

Chemical:

Specific chemicals:

Mixtures:

Audience:

Topics addressed:

\section{Summary:}

151

NIOSH [2005]. International Chemical Safety Cards

(ICSC): U.S. national version. [www.cdc.gov/niosh/ipcs/ nicstart.html].

Web page

No

General-overview

Abrasives, cleaning agents, coolants, corrosives, heavy metals/inorganic compounds, organic dyes, particulates, pesticides, petroleum products \& lubricants, plastics and resins, PCBs, solvents, other: comprehensive list of chemicals used in occupational settings

There are currently cards for over 1500 chemicals.

No

General

C Hazard identification

C.1 Risk phrases, hazard symbols, skin designations

C.2 Tables/charts/lists of hazards for specific chemicals

E Risk management

E.1 Overview of skin exposure control options

E.3 "Best practices"/guidelines/recommendations

E.3.C Work practice/administration controls

E.3.D PPE and PPE rules

E.4 Guidelines/recommendations for postexposure skin decontamination

ICSC Project is an undertaking of the International Programme on Chemical Safety (IPCS). The IPCS is a joint activity of three cooperating international organizations: the United Nations Environment Programme (UNEP), the ILO, and the WHO. Each ICSC summarizes essential health and safety information on chemicals for their use by workers and employers in factories, agriculture, construction, and other work places. They consist of a series of standard phrases, mainly summarizing health and safety information collected, verified and peer reviewed by internationally recognized experts, taking into account advice from manufacturers and poison control centers. 
The U.S. national version of the ICSCs cited here has been modified by the National Institute for Occupational Safety and Health (NIOSH) to include the following:

- OSHA permissible exposure limits (PELs).

- NIOSH recommended exposure limits (RELs).

- Immediately dangerous to life and health values (IDLHs).

- Links to the Appendices in the NIOSH Pocket Guide to Chemical Hazards.

Each card briefly lists the routes of exposure, potential acute skin hazards, and symptoms for each specific chemical, as well as general prevention and first-aid measures.

Article ID:

\section{Citation:}

Resource type:

Educational materials:

Number of references:

Industries/occupations:

Specific process:

Chemical:

Specific chemicals:

Mixtures:

Audience:

Topics addressed:

\section{2}

NIOSH [2005]. NIOSH pocket guide to chemical hazards. Cincinnati, OH: U.S. DHHS, PHS, CDC, NIOSH, DHHS (NIOSH) Publication No. 97-140.

Technical publication/report

No

0

Abrasives, cleaning agents, coolants, corrosives, fiberglass and other fibers, food products, hand cleansers, heavy metals/inorganic compounds, latex, nanoparticles, organic dyes, particulates, pesticides, petroleum products \& lubricants, plastics and resins, PAHs, PCBs, rubber additives, soaps and detergents, solvents

398 chemicals included

No

General

C Hazard identification

C.2 Tables/charts/lists of hazards for specific chemicals

E Risk management

E.3 "Best practices"/guidelines/recommendations

E.3.D PPE and PPE rules

E.4 Guidelines/recommendations for postexposure skin decontamination 
Summary:

Article ID:

Citation:

Resource type:

Educational materials:

Number of references:

Industries/occupations:

Specific process:

Chemical:

Specific chemicals:

Mixtures:

Audience:

Topics addressed:
The NIOSH Pocket Guide to Chemical Hazards is a source of general industrial hygiene information on several hundred chemicals/classes for workers, employers, and occupational health professionals. It provides exposure limits, exposure routes, respirator recommendations, PPE suggestions, and first aid for many of the 398 chemicals reviewed. It presents key information and data in abbreviated or tabular form for chemicals or substance groupings (e.g. cyanides, fluorides, manganese compounds) that are found in the work environment. This portable reference book helps in responding to workplace emergencies and preventing exposures to workers. It is designed to help users recognize and control occupational chemical hazards. It does not present data analyses.

It is available online, on a CD, or as a hard copy, spiral bound document. It contains chemical-specific information on the skin designation (denoted as [skin]), which indicates the potential for dermal absorption. Skin exposure should be prevented as necessary through the use of good work practices and gloves, coveralls, goggles, and other appropriate equipment.
153

NIOSH [2005]. [www.cdc.gov/niosh/homepage.html].

Web site

Yes

General—overview, Agricultural, Cleaning/Janitorial/Maid, Service-Medical

General-overview, cleaning agents, coolants, corrosives, fiberglass and other fibers, heavy metals/inorganic compounds, latex, nanoparticles, organic dyes, particulates, pesticides, petroleum products \& lubricants, plastics and resins, PAHs, PCBs, rubber additives, soaps and detergents, solvents

No Professional

A Overview

A.1 Occurrence of skin exposures in the workplace

A.2 Health hazards resulting from skin exposure to chemicals 
A.3 Investigation, intervention, and control of occupational skin exposures

A.5 Dermal regulations and skin notations

B Surveillance and clinical aspects

B.1 Surveillance study reporting incidences of occupational skin exposures

B.1.A Skin exposure major focus

B.2 Loss of workdays and impact on productivity

C Exposure characterization

C.1 Workplace factors associated with harmful skin exposures

C.2 Description of factors influencing exposure conditions

C.2.A Exposure intensity/frequency/duration

C.2.B Exposure concentration

C.2.C Skin area affected

C.4 Direct methods to measure exposure

C.4.A Surfaces

D Hazard identification

D.1 Potential health effects resulting from specific chemicals

D.1.A Irritant contact dermatitis

D.1.B Allergic contact dermatitis/sensitization

D.1.C Systemic toxicity

F Risk management

F.1 Exposure control strategies

F.1.A Substitution

F.1.B Engineering controls

F.1.C Work practice/Administrative controls

F.1.D PPE and PPE rules

F.1.E Skin management, barrier creams, moisturizers, cleansers, and rubs

Summary:

$\mathrm{NIOSH}$ is the federal agency responsible for conducting research and making recommendations for the prevention of work-related injury and illness. NIOSH's Web site has a variety of Web pages with information on dermal exposure to chemicals. Many of these resources can be accessed through the NIOSH safety and health topic page, "Skin Exposures and Effects." This Web page contains links to many of the NIOSH resources on dermal exposure, including the NORA Dermal Exposure Research Program 
(DERP), as well as updates on ongoing research and conferences. Information on dermal exposure can also be accessed by chemical or by industry and occupation and then examined for dermal exposure-related information.

Additional NIOSH resources on dermal exposure available from this Web site include:

- The Registry of Toxic Effects of Chemical Substances (RTECS) is a toxicological database of chemical data extracted from the open scientific literature. For each chemical, six types of toxicity data are included in the file: (1) primary irritation, (2) mutagenic effects, (3) reproductive effects, (4) tumorigenic effects, (5) acute toxicity, and (6) other multiple dose toxicity. Where available, it includes skin and eye irritation data. A subscription from one of RTECS' database vendors, listed on the Web page, is necessary to access the database.

- NIOSHTIC 2 is a searchable bibliographic database of occupational safety and health publications, documents, grant reports, and journal articles supported in whole or in part by NIOSH.

- The CPC Database can be searched by chemical, and for each chemical the user can find information on whether skin contact should be avoided and a list of recommended protective clothing barriers.

- International Chemical Safety Cards (ISCS) is a searchable database of basic health and safety information on (ultimately) 2000 chemicals and can be searched by chemical for potential dermal hazards.

- The NIOSH Pocket Guide to Chemical Hazards is a searchable source of general industrial hygiene information on several hundred chemicals/classes for workers, employers, and occupational health professionals, including information on routes of exposure, target organs, symptoms, and first-aid procedures.

- The Skin Permeation Calculator can be used to calculate the skin permeation coefficient $(\mathrm{Kp})$, a measure of the conductance of skin to a particular chemical from a particular vehicle.

- The National Occupational Research Agenda (NORA) Allergic and Irritant Dermatitis (AID) Team. NORA, which is a framework to guide occupational safety and health research into the next decade, created the AID Team to promote research in this area. 
- Proceedings of the International Conference on Occupational and Environmental Exposures of Skin to Chemicals: Science \& Policy, September 2002.

- Worker Health Chart Book 2004: Chapter 2, "Fatal and Nonfatal Injuries, and Selected Illnesses and Conditions, Skin Diseases and Disorders," presents national surveillance data on skin diseases and disorders.

- Occupational Dermatoses: A Program for Physicians is a slide show that presents an overview of occupational dermatitis, including both surveillance data and photographs of different types of dermatitis.

- A NIOSH Alert on Preventing Allergic Reactions to Natural Rubber Latex in the Workplace is a comprehensive document that provides information on the recognition, evaluation, and control of exposure to natural latex products. It includes a list of a number of products found in the workplace that may contain latex.

- Control of Exposure to Perchloroethylene in Commercial Dry Cleaning is a guide which includes a description of methods that can be used for exposure control.

Article ID:

Citation:

Resource type:

Educational materials:

Number of references:

Industries/occupations:

Specific process:

Chemical:
National Library of Medicine (NLM) [2005]. Toxicology Data Network (TOXNET) — databases on toxicology, hazardous chemicals, environmental health, and toxic releases. [http://toxnet.nlm.nih.gov/].

Web site

No

Agricultural, Beauty/Cosmetology, Cleaning/Janitorial/ Maid, Construction, Forestry/Fisheries, ManufacturingChemical, Manufacturing-Other, Medical Services, Mining, Service—Food, Service-Medical, Service—Other, Transportation/Communications/Utility

Haz-Map is searchable by job name and job task

Abrasives, cleaning agents, coolants, corrosives, fiberglass and other fibers, heavy metals/inorganic compounds, latex, nanoparticles, organic dyes, particulates, pesticides, petroleum products \& lubricants, plastics and resins, PAHs, PCBs, rubber additives, solvents

Specific chemicals: 
Mixtures:

Audience:

Topics addressed:

Summary:
No

Professional

A Overview

A.1 Occurrence of skin exposures in the workplace

A.2 Health hazards resulting from skin exposure to chemicals

A.3 Investigation, intervention, and control of occupational skin exposures

A.5 Dermal regulations and skin notations

B Surveillance and clinical aspects

B.4 Clinical protocols for recognition of skin exposure health effects

C Exposure characterization

C.1 Workplace factors associated with harmful skin exposures

D Hazard identification

D.1 Potential health effects resulting from specific chemicals

D.1.A Irritant contact dermatitis

D.1.B Allergic contact dermatitis/sensitization

D.1.C Systemic toxicity

D.1.D Other health effects

D.1.E Contribution to overall exposure

D.2 Summaries of health effects, dose-response relationships

F Risk management

F.1 Exposure control strategies

F.1.C Work practice/Administrative controls

F.1.D PPE and PPE rules

TOXNET is a cluster of databases covering toxicology, hazardous chemicals, environmental health and related areas. It is managed by the Toxicology and Environmental Health Information Program (TEHIP) in the Division of Specialized Information Services (SIS) of the NLM. TOXNET provides free access to a variety toxicology databases, including those described below:

- Haz-Map: an occupational toxicology database designed primarily for health and safety professionals, but also for consumers seeking information about the health effects of exposure to approximately 1,000 chemicals and biological agents at work. Haz-Map links jobs and hazardous tasks with occupational 
diseases and their symptoms (see ID 155 for more information). (http://hazmap.nlm.nih.gov/ hazmapadv.html)

- HSDB: a comprehensive, peer-reviewed resource for toxicology information on over 4,900 potentially hazardous chemicals. HSDB also provides information on emergency handling procedures, industrial hygiene, environmental fate, human exposure, detection methods, and regulatory requirements. (http://toxnet.nlm.nih.gov/cgi-bin/sis/ htmlgen?HSDB)

- TOXLINE: a bibliographic database providing comprehensive coverage of the biochemical, pharmacological, physiological, and toxicological effects of drugs and other chemicals from 1965 to the present.

- Integrated Risk Information System (IRIS): a database from the USEPA that contains health risk information on over 500 chemicals. IRIS risk assessment data have been scientifically reviewed by scientists and represents consensus.

- ChemIDplus: a database providing access to a variety of databases used for the identification of chemical substances cited in NLM databases. ChemIDplus is searchable by chemical name, synonym, CAS registry number, molecular formula, classification code, locator code, and structure. Links to available databases are provided. ChemIDplus contains over 379,000 chemical records, of which over 257,000 include chemical structures.

- Wireless Information System for Emergency Responders (WISER): a system designed to assist first responders in hazardous material incidents. It is available as a web-based, windows-based, or PDA application. It provides a wide range of information on hazardous substances, including substance identification support, physical characteristics, human health information, and containment and suppression advice.

Article ID:

Citation:

Resource type:

Educational materials:
155

NLM [2005]. Hazardous Substances Data Bank (HSDB) [http://toxnet.nlm.nih.gov/cgi-bin/sis/htmlgen? HSDB].

Web page

No 


\section{Number of references:}

Industries/occupations:

Specific process:

Chemical:

Specific chemicals:

Mixtures:

Audience:

Topics addressed:

Summary:
Agricultural, Beauty/Cosmetology, Cleaning/ Janitorial/Maid, Construction, Forestry/Fisheries, Manufacturing-Chemical, Manufacturing-Medical Services, Mining, Service-Food, Service-Medical, Service— Transportation/Communications/Utility

Abrasives, cleaning agents, coolants, corrosives, fiberglass and other fibers, heavy metals/inorganic compounds, latex, nanoparticles, organic dyes, particulates, pesticides, petroleum products \& lubricants, plastics and resins, PAHs, PCBs, rubber additives, solvents

No

Professional

A Overview

A.5 Dermal regulations and skin notations

B Surveillance and clinical aspects

B.1 Surveillance study reporting incidences of occupational skin exposures

B.1.A Skin exposure major focus

B.1.B Skin exposure minor focus

C Exposure characterization

C.1 Workplace factors associated with harmful skin exposures

D Hazard identification

D.1 Potential health effects resulting from specific chemicals

D.1.A Irritant contact dermatitis

D.1.B Allergic contact dermatitis/sensitization

D.1.C Systemic toxicity

D.1.D Other health effects

F Risk management

F.1 Exposure control strategies

F.1.C Work practice/Administrative controls

F.1.D PPE and PPE rules

The HSDB is a toxicology data file on the NLM TOXNET. It focuses on the toxicology of potentially hazardous chemicals. It includes information on human exposure, industrial hygiene, emergency handling procedures, environmental fate, regulatory requirements, and related 
areas. All data are referenced and derived from a core set of books, government documents, technical reports and selected primary journal literature. HSDB is peer reviewed by the Scientific Review Panel (SRP), a committee of experts in the major subject areas within HSDB's topic areas. HSDB is organized into individual chemical records and contains over 4,900 records. The following broad groupings of information are provided, if available, for each chemical:

- Human Health Effects

- Emergency Medical Treatment

- Animal Toxicity Studies

- Metabolism/Pharmacokinetics

- Pharmacology

- Environmental Fate/Exposure

- Chemical/Physical Properties

- Chemical Safety \& Handling

- Occupational Exposure Standards

- Manufacturing/Use Information

- Laboratory Methods

- Special References

- Synonyms and Identifiers

- Administrative Information

Article ID:

\section{Citation:}

\section{Resource type:}

Educational materials:

Number of references:

Industries/occupations:

Specific process:

Chemical:

Specific chemicals:

Mixtures:

Audience:

Topics addressed:
156

National Ready Mixed Concrete Association (NRMCA) [2006]. [www.nrmca.org].

Web site

Yes

Construction

Corrosives

Portland cement

No

General

A Overview

A.1 Occurrence of skin exposures in the workplace

E Risk management 
Summary:

Article ID:

Citation:

Resource type:

Educational materials:

Number of references:

Industries/occupations:

Specific process:

Chemical:

Specific chemicals:

Mixtures:

Audience:

Topics addressed:
E.1 Overview of skin exposure control options

E.3 "Best practices"/guidelines/recommendations

E.3.C Work practice/administration controls

E.3.D PPE and PPE rules

E.3.E Skin management, barrier creams, moisturizers, cleansers, and rubs

The NRMCA is an industrial organization for the readymix concrete industry. Through their Web site's E-Store, there are several resources available on dermal exposure hazards and controls associated with ready-mix concrete. These include:

- Cement Burn Awareness Kit (training material)

- Cement Burn Warning (Mini Poster)

- Safe Drum Cleaning (video)

157

Ness SA [1994]. Surface and dermal monitoring for toxic exposures. New York: Wiley \& Sons.

Book/monograph, whole

No

1303

General-overview

General-overview, heavy metals/inorganic compounds, organic dyes, pesticides, PCBs, other: TCDD

Sampling methods listed for dozens of specific chemicals

No

Professional

A Overview

A.2 Health hazards resulting from skin exposure to chemicals

A.3 Investigation, intervention, and control of occupational skin exposures

C Exposure characterization

C.2 Description of factors influencing exposure conditions

C.2.A Exposure intensity/frequency/duration

C.2.B Exposure concentration

C.2.C Skin area affected 
Summary:

\begin{tabular}{|c|c|}
\hline C.2.E & Uptake \\
\hline C. 3 & $\begin{array}{l}\text { Checklists/questionnaires to quantify skin } \\
\text { exposure incidences }\end{array}$ \\
\hline C. 4 & Direct methods to measure exposure \\
\hline C.4.A & Surfaces \\
\hline C.4.B & Skin \\
\hline $\mathrm{F}$ & Risk management \\
\hline F.1 & Exposure control strategies \\
\hline F.1.D & PPE and PPE rules \\
\hline
\end{tabular}

This definitive book was one of the first comprehensive guides to surface and dermal sampling methods. This book is divided into four parts, with accompanying chapters in each part, as described below:

Part I-Chemical Hazards

Ch. 1 Identifying Chemicals as Hazards

Ch. 2 Factors Affecting Chemical Permeation

Ch. 3 Chemical Protective Clothing

Part II—Developing Strategies for Sampling

Ch. 4 Assessment of Workplace Exposures

Ch. 5 Assessment of Community Exposures

Part III-Surface Monitoring

Ch. 6 Introduction to Surface Monitoring

Ch. 7 Surface Sampling for Chemicals

Ch. 8 Surface Sampling for Microorganisms

Ch. 9 Surface Sampling for Low Level Radiation

Ch. 10 Decontamination

Part IV—Dermal Sampling Techniques

Ch. 11 Introduction to Dermal Monitoring

Ch. 12 Skin Sampling Methods, Part 1, Wiping Swabbing and Washing

Ch. 13 Skin Sampling Methods, Part 2, Direct Reading

Ch. 14 Pad Dosimetry Methods

Ch. 15 Clothing for Dosimetry and Protection

The book also includes appendices on methods and studies of amines, metals, polychlorinated biphenyls, tetrachlorodibenzodioxins, and pesticides.

Article ID:

158

Citation:

Ness SA [2000]. Surface and dermal monitoring. In:

Meyers RA, ed. Encyclopedia of analytical chemistry. New

York: Wiley, pp. 4824-43. 
Resource type:

Educational materials:

Number of references:

Industries/occupations:

Specific process:

Chemical:

Specific chemicals:

Mixtures:

Audience:

Topics addressed:
Book/monograph, chapter

No

61

General—overview

Fiberglass and other fibers, heavy metals/inorganic compounds, pesticides, PCBs

No

Professional

C Exposure characterization

C.2 Description of factors influencing exposure conditions

C.2.C Skin area affected

C.2.E Uptake

C.3 Checklists/questionnaires to quantify skin exposure incidences

C.4 Direct methods to measure exposure

C.4.A Surfaces

C.4.B Skin

C.5 Exposure modeling

This chapter provides an overview of current methods used to perform surface and dermal monitoring for chemicals. It describes methods for measuring surface contamination, as well as discusses surface sampling media and sampling strategies. It also covers dermal monitoring methods which directly assess chemical contamination on a worker's skin or clothing. In addition, it contains a table listing guidelines and standards for surface sampling results for 20 different chemicals or chemical groups.

Article ID: 159

Citation:

Resource type:

Educational materials:

Number of references:

Industries/occupations:

Specific process:
Nielsen J B, Grandjean P [2004]. Criteria for skin notation in different countries. Am J Ind Med 45(3):275-80.

Journal article—review, meta-analysis

No

19 
Chemical:

Specific chemicals:

Mixtures:

Audience:

Topics addressed:

Summary:
Solvents

Ethylamine, Cyanamide, Methacrylic acid, Sodium azide, Acroleine, Xylene, N-hexane, Toluene, Perchloroethylene, Benzene, 1,3,-butadiene, Ethylbenzene,

No

Professional

A Overview

A.5 Dermal regulations and skin notations

This paper compared skin notations on lists of exposure limits for industrial chemicals in six countries - the U.S., Netherlands, Denmark, Poland, Slovakia, and Germany-and found that one-third of industrial chemicals listed had a skin notation. Differences in criteria for assigning skin notations did not explain discrepancies between countries that otherwise had comparable occupational exposure limits (OELs).

Article ID:

160

Citation:

Resource type:

Educational materials:

Number of references:

Industries/occupations:

Specific process:

Chemical:

Specific chemicals:

Mixtures:

Audience:

Topics addressed:

Summary:
Nygren O [2002]. New approaches for assessment of occupational exposure to metals using on-site measurements. J Environ Monit 4(5):623-27. Journal article-review, meta-analysis

No

10

Heavy metals/inorganic compounds

No

Professional

C Exposure characterization

C.4 Direct methods to measure exposure

C.4.A Surfaces

C.4.B Skin

This article compares the accuracy of XRF florescent tracers to the traditional use of membrane filters followed by laboratory analysis. Tests were performed using dust, cobalt, nickel, and molydemum. 
Citation:

Resource type:

Educational materials:

Number of references:

Industries/occupations:

Specific process:

Chemical:

Specific chemicals:

Mixtures:

Audience:

Topics addressed:

Summary:

Article ID:

Citation:

Resource type:

Educational materials:

Number of references:

Industries/occupations:

Specific process:

Chemical:

Specific chemicals:
OSHA [2005]. Sampling for surface contamination. Washington, DC: U.S. DOL, OSHA, TED 1-0.15A.

Guideline

No

7

Plastics and resins, other: Isocyanates

No

Professional

A Overview

A.5 Dermal regulations and skin notations

C Exposure characterization

C.4 Direct methods to measure exposure

C.4.A Surfaces

C.4.B Skin

Section II, Chapter 2 of the OSHA Technical Manual describes surface sampling protocols for OSHA inspectors but is applicable to a wider audience. Substances with skin notations are listed in an appendix.

162

OSHA [2005]. [www.osha.gov].

Web site

No

General—overview, Agricultural, Cleaning/Janitorial/

Maid, Construction, Manufacturing-Chemical,

Manufacturing_-Other, Medical Services, Mining,

Service-Medical

General-overview, coolants, corrosives, heavy metals/ inorganic compounds, pesticides, petroleum products \& lubricants, plastics and resins, solvents

Acrylonitrile, benzene, 1, 3-butadiene, dry cleaning chemicals, CrVI, formaldehyde, isocyanates, methylene chloride, acrylonitrile, benzene, 1, 3-butadiene, dry cleaning chemicals, CrVI, formaldehyde, isocyanates, methylene chloride, among others. 
Mixtures:

Audience:

Topics addressed:
Summary:
No

Professional

A Overview

A.1 Occurrence of skin exposures in the workplace

A.2 Health hazards resulting from skin exposure to chemicals

A.5 Dermal regulations and skin notations

C Exposure characterization

C.1 Workplace factors associated with harmful skin exposures

C.2 Description of factors influencing exposure conditions

C.2.A Exposure intensity/frequency/duration

C.2.B Exposure concentration

C.2.C Skin area affected

C.4 Direct methods to measure exposure

C.4.A Surfaces

C.4.B Skin

C.4.C Biomonitoring

E Risk assessment

E.1 Guidelines for risk assessment or analysis

E.1.A Localized health effects

E.1.B Systemic health effects

F Risk management

F.1 Exposure control strategies

F.1.A Substitution

F.1.B Engineering controls

F.1.C Work practice/Administrative controls

F.1.D PPE and PPE rules

F.1.E Skin management, barrier creams, moisturizers, cleansers, and rubs

OSHA is the federal agency under the Department of Labor that publishes and enforces safety and health regulations for most businesses and industries in the United States. In recent years, OSHA's focus has been on enforcement as well as outreach, education, compliance assistance, and partnerships and cooperative programs. The OSHA Web site provides information and links to information on dermal exposure, including: 
- Health and Safety Topics: This Web page includes a link to OSHA's dermal exposure Web page as well as Web pages for specific chemicals with dermal exposure potential, such as acrylonitrile; benzene; 1, 3- butadiene; dry cleaning chemicals; CrVI; formaldehyde; isocyanates; methylene chloride; metalworking fluids; and solvents. There are also links to information on surface contamination associated with chemicals that have skin designations, and an up-to-date list of the OSHA standards that address dermal exposure. Additional topics include how to recognize hazardous dermal exposures, how to evaluate dermal exposures, and how to control dermal exposures including personal protective equipment.

- OSHA Technical Manual: This manual is used by OSHA compliance officers as a reference for technical information on occupational safety and health issues. It includes a number of chapters with information relevant to dermal exposure, including dermal exposure hazards specific to chemicals or processes, methods for sampling for surface contamination, chemical protective clothing guidelines, and a list of substances listed with skin notations or designations by ACGIH TLVs and/or OSHA PELs.

- Evaluation Guidelines for Surface Sampling Methods: A document developed to provide chemists with a uniform means for evaluating surface sampling methods with regards to sampling media, sampling techniques, and sample preparation for analysis.

- "Chemical Sampling Information": This Web page provides data on a large number of chemicals that may be encountered in industrial hygiene investigations. It is meant as a basic reference for OSHA personnel. For select chemicals it contains OSHA wipe sampling methods.

- OSHA standards: Dermal exposures are addressed in specific standards for general industry, shipyard employment, marine terminals, the construction industry and for the identification, classification, and regulation of carcinogens, in addition to being covered in Section 5(a)(1) of the OSH Act (the General Duty Clause) which require an employer to "furnish to each of his employees employment and a place of employment that is free from recognized hazards that are causing or are likely to cause death or serious physical harm to his employees." Below is a highlight of OSHA standards and directives (instructions for compliance officers). This is only a 
partial list of references to skin exposure in OSHA standards, guidelines, and chemical sampling methods. For more and up-to-date information, see this Web site.

General Industry (29 CFR 1910)

1910 Subpart H, Hazardous materials

1910.120, Hazardous waste operations and emergency response

1910 Subpart I, Personal protective equipment

1910 Subpart Z, Toxic and hazardous substances

1910.1028, Benzene

1910.1044, 1,2-dibromo-3-chloropropane

1910.1045, Acrylonitrile

1910.1048, Formaldehyde

1910.1050, Methylenedianiline.

1910.1051, 1.3-Butadiene

1910.1052, Methylene chloride

1910.1200, Hazard communication

Shipyard Employment (29 CFR 1915)

1915 Subpart I, Personal protective equipment

1915 Subpart I Appendix A, Non-mandatory guidelines for hazard assessment, personal protective equipment (PPE) selection, and PPE training program

Marine Terminals (29 CFR 1917)

1917 Subpart B, Marine terminal operations

1917.28 Hazard communication

Construction (29 CFR 1926)

1926 Subpart D, Occupational health and environmental controls

1926.60, Methylenedianiline

1926.65, Hazardous waste operations and emergency response

Identification, Classification, and Regulation of Carcinogens (29 CFR 1990.103, Definitions)

Directives:

Enforcement Procedure for Occupational Exposure to Formaldehyde. Compliance directives (CPL) 02-02-052 [CPL 2-2.52], (1990, November 20). Benzidine-Based Dyes: Direct Black 38, Direct Brown 95 and Direct Blue 6 Dyes. CPL 02-02-027 [CPL 2-2.27], (1980, February 22). 
Citation:

\section{Resource type:}

Educational materials:

Number of references:

Industries/occupations:

Specific process:

Chemical:

Specific chemicals:

Mixtures:

Audience:

Topics addressed:

Summary:
Oppl R, Kalberlah F, Evans PG, van Hemmen JJ. A toolkit for dermal risk assessment and management: An overview. Ann Occup Hyg 47(8):629-40.

Journal article—review, meta-analysis

No

15
No

Professional

C Exposure characterization

C.5 Exposure modeling

E Risk assessment

E.1 Guidelines for risk assessment or analysis

E.1.A Localized health effects

E.1.B Systemic health effects

F Risk management

F.2 Protocols for risk management

F.2.A Development of exposure reduction goal (qualitative or quantitative)

This article is the 2nd article of a six-part series on RISKOFDERM, a tool for conducting risk assessments. The series was published in the Annals of Occupational Hygiene in 2003 . The following briefly summarizes each paper in the series:

1. ID 212-Outlines a "toolkit" for conducting dermal occupational risk assessment.

2. ID 163-Describes the assumptions in the toolkit and describes an approach to exposure assessment used by the toolkit.

3. ID 139-Describes the determinants relevant for dermal exposure models in the scope of regulatory risk assessment.

4. ID 219-Describes how default dermal exposure values can be adjusted for specific work situations.

5. ID 100-Describes the derivation of the toolkit's default task-based dermal exposure values.

6. ID 193-Describes the development of "intrinsic toxicity" (IT) scores used for hazard characterization. 


\begin{tabular}{|c|c|c|}
\hline Article ID: & \multicolumn{2}{|l|}{164} \\
\hline Citation: & \multicolumn{2}{|c|}{$\begin{array}{l}\text { Oregon Department of Human Services (ORDHS) [2005]. } \\
\text { Oregon Worker Illness and Injury Prevention Program } \\
\text { (OWIIPP). [http://oregon.gov/DHS/ph/owiipp/index.shtml]. }\end{array}$} \\
\hline Resource type: & \multicolumn{2}{|c|}{ Web site } \\
\hline Educational materials: & \multicolumn{2}{|l|}{ No } \\
\hline \multicolumn{3}{|l|}{ Number of references: } \\
\hline Industries/occupations: & \multicolumn{2}{|c|}{ General-overview } \\
\hline \multicolumn{3}{|l|}{ Specific process: } \\
\hline Chemical: & \multicolumn{2}{|c|}{ General-overview, latex, rubber additives } \\
\hline \multicolumn{3}{|l|}{ Specific chemicals: } \\
\hline Mixtures: & \multicolumn{2}{|l|}{ No } \\
\hline Audience: & \multicolumn{2}{|c|}{ Professional } \\
\hline \multirow[t]{9}{*}{ Topics addressed: } & $\mathrm{B}$ & Surveillance and clinical aspects \\
\hline & B. 1 & $\begin{array}{l}\text { Surveillance study reporting incidences of } \\
\text { occupational skin exposures }\end{array}$ \\
\hline & B.1.A & Skin exposure major focus \\
\hline & B. 2 & Loss of workdays and impact on productivity \\
\hline & B.3 & $\begin{array}{l}\text { Surveillance study protocols/procedures for } \\
\text { gathering data }\end{array}$ \\
\hline & $\mathrm{F}$ & Risk management \\
\hline & F.1 & Exposure control strategies \\
\hline & F.1.A & Substitution \\
\hline & F.1.C & Work practice/Administrative controls \\
\hline Summary: & \multicolumn{2}{|c|}{$\begin{array}{l}\text { The OWIIPP is working to identify and prevent targeted } \\
\text { occupational illnesses and injuries in Oregonians. } \\
\text { Workplace dermatitis and latex allergies are two of their } \\
\text { targeted occupational illnesses. }\end{array}$} \\
\hline
\end{tabular}

Article ID: $\quad 165$

\begin{tabular}{|c|c|}
\hline Citation: & $\begin{array}{l}\text { OR-OSHA [2006]. [www.cbs.state.or.us/external/osha/ } \\
\text { index.html]. }\end{array}$ \\
\hline Resource type: & Web site \\
\hline Educational materials: & Yes \\
\hline \multicolumn{2}{|l|}{ Number of references: } \\
\hline Industries/occupations: & General-overview, Agricultural \\
\hline \multicolumn{2}{|l|}{ Specific process: } \\
\hline Chemical: & General—overview, latex, pesticides, PCBs \\
\hline
\end{tabular}

Specific chemicals: 
Mixtures:

Audience:

Topics addressed:

Summary:

Article ID:

Citation:
No

General

A Overview

A.2 Health hazards resulting from skin exposure to chemicals

A.3 Dermal regulations and skin notations

B Exposure characterization

B.1 Job/tasks, industries/processes, or chemicals associated with skin exposures

B.2 Factors that influence exposure conditions

B.2.A Exposure intensity/frequency

B.2.B Exposure controls

E Risk management

E.1 Overview of skin exposure control options

E.3 "Best practices"/guidelines/recommendations

E.3.C Work practice/administration controls

E.3.D PPE and PPE rules

E.3.E Skin management, barrier creams, moisturizers, cleansers, and rubs

OR-OSHA, a division of the Oregon Department of Consumer and Business Services, enforces Oregon state workplace safety and health rules. Their Web site has a variety of resources, including pamphlets and brochures, on working safely with agricultural chemicals. Although these do not exclusively address skin exposure, they do address skin exposures in terms of overall routes of exposure to agricultural chemicals. Examples include:

- Safe practices when working around hazardous agricultural chemicals (brochure).

- Clothes washing for pesticide handlers (magnet).

- IH for the non-IH (workbook).

- Audio-visual library with several videos on skin exposure.

- Training materials for basic health and safety, including identifying and controlling hazards and personal protective equipment. 


\section{Resource type:}

Educational materials:

Number of references:

Industries/occupations:

Specific process:

Chemical:

Specific chemicals:

Mixtures:

Audience:

Topics addressed:

Summary: of chemicals. [www.oecd.org/findDocument/0,2350, en_2649_34377_1_1_1_1_1,00.html].

Web page

No

General—overview

No

Professional

D Hazard identification

D.3 Characterization protocols

D.3.A Corrosivity

D.3.B Irritation potential

D.3.C Sensitization potential

D.3.D Potential to cause systemic effects

D.3.E Measurement of skin permeation rates and reservoir effects

The Guidelines for the Testing of Chemicals are a basic set of tools that are designed for use in regulatory safety testing, subsequent chemical product notification, and chemical registration. These are only guidelines. The existing guidelines are periodically updated and new guidelines are also added regularly. The dermal related guidelines listed below can be downloaded from the OECD site.

- OECD Guidelines for the Testing of Chemicals, Sections 1-5; Health Effects, Section 4

- 402 Acute Dermal Toxicity (updated guideline, adopted February 24, 1987)

- 404 Acute Dermal Irritation/Corrosion (updated guideline, adopted April 24, 2002)

- 406 Skin Sensitisation (updated guideline, adopted July 17, 1992)

- 410 Repeated Dose Dermal Toxicity: 21/28-day Study (original guideline, adopted May 12, 1981)

- 411 Subchronic Dermal Toxicity: 90-day Study (original guideline, adopted May 12, 1981)

- 427 Skin Absorption: In Vivo Method (original guideline, adopted April 13, 2004) 
- 428 Skin Absorption: In Vitro Method (original guideline, adopted April 13, 2004)

- 429 Skin Sensitization: Local Lymph Node Assay (updated guideline, adopted April 24, 2002)

- 430 In Vitro Skin Corrosion: Transcutaneous Electrical Resistance Test (TER) (original guideline, adopted April 13, 2004)

- 431 In Vitro Skin Corrosion: Human Skin Model Test (original guideline, adopted April 13, 2004)

- 434 Acute Dermal Toxicity-Fixed Dose Procedure, Draft New Guideline (May 2004) (deadline for public comments passed: July 16,2004$)$

Chemical Testing Guidelines

- No. 9: Guidance Document for the Conduct of Studies of Occupational Exposure to Pesticides During Agricultural Application

- No. 13: Detailed Review Document on Classification Systems for Sensitizing Substances in OECD Member Countries

- No. 16: Detailed Review Document on Classification Systems for Skin Irritation/Corrosion in OECD Member Countries

Article ID:

\section{Citation:}

Resource type:

Educational materials:

Number of references:

Industries/occupations:

Specific process:

Chemical:

Specific chemicals:

Mixtures:

Audience:

Topics addressed:
167

OECD [2006]. OECD's database on chemical risk assessment models. [http://webdomino1.oecd.org/comnet/ env/models.nsf].

Web page

No

General—overview

General—overview

No

Professional

C Exposure characterization

C.5 Exposure modeling

D Hazard identification 
D.3 Characterization protocols

D.3.F QSARs—-development, validation, and application

Summary:

This searchable database includes information on models (computerized or capable of being computerized) that are used by OECD member governments and industry to predict health or environmental effects, exposure potential, and possible risks. The database can be searched by route of exposure and will list all dermal models. These are generic exposure models and not specific to occupational settings. The methods described here have not been evaluated or validated by OECD, and no endorsement of the methods by OECD should be inferred by the inclusion of certain methods in this Web page.

Article ID:

\section{8}

Citation:

Resource type:

Educational materials:

Number of references:

Industries/occupations:

Specific process:

Chemical:

Specific chemicals:

Mixtures:

Audience:

Topics addressed:
Packham CL [1996]. Risk assessment and exposure control in an occupational setting. Curr Probl Dermatol 25:133-44. Journal article—primary

No

0
No

Professional

E Risk assessment

E.1 Guidelines for risk assessment or analysis

E.1.A Localized health effects

E.1.B Systemic health effects

E.2 Example of risk assessments

F Risk management

F.1 Exposure control strategies

F.1.B Engineering controls

F.1.C Work practice/Administrative controls

F.1.D PPE and PPE rules

F.2 Protocols for risk management

F.2.B Development of approach to achieve exposure reduction goal 
Summary:

This paper presents a process by which managers can identify dermal risks of greatest concern, a necessary step prior to invoking risk management strategies.

Article ID:

169

Citation:

Resource type:

Educational materials:

Number of references:

Industries/occupations:

Specific process:

Chemical:

Specific chemicals:

Mixtures:

Audience:

Topics addressed:
Packham CL [1999]. Essentials of occupational skin management. Southport, UK: The Limited Edition Press.

Book/monograph, whole

No

95

General-overview, Agricultural, Beauty/Cosmetology, Cleaning/Janitorial/Maid, Construction, ManufacturingChemical, Manufacturing-Other, Service-Food, Service-Medical, Transportation/Communications/ Utility, Other: aerospace, dentistry, pharmaceuticals, printing, textiles,

General-overview, plastics and resins, rubber additives

No

General

A Overview

A.1 Occurrence of skin exposures in the workplace

A.2 Health hazards resulting from skin exposure to chemicals

A.3 Dermal regulations and skin notations

B Exposure characterization

B.1 Job/tasks, industries/processes, or chemicals associated with skin exposures

B.2 Factors that influence exposure conditions

B.2.A Exposure intensity/frequency

B.2.B Exposure controls

C Hazard identification

C.3 Protocols/checklists to identify skin hazards in the workplace

D Risk assessment

D.1 Protocols/checklists to identify exposure risk

E Risk management

E.1 Overview of skin exposure control options 


\section{Summary:}

E.2 Protocols/checklists to monitor potential exposures

E.3 "Best practices"/guidelines/recommendations

E.3.A Substitution

E.3.B Engineering controls

E.3.C Work practice/administration controls

E.3.D PPE and PPE rules

E.3.E Skin management, barrier creams, moisturizers, cleansers, and rubs

E.4 Guidelines/recommendations for postexposure skin decontamination

This comprehensive book combines elements of dermatology, occupational hygiene, and engineering and provides practical examples and solutions. It is clearly written and appears to be useful to both professionals and other users. Although the book is more focused on practical application rather than presenting scientific argument, it addresses many complex subjects, such as uptake, in a straightforward manner. Chapter headings are not self-evident, but the book contains an extensive index. Chapters are:

1. Dermatological Engineering

2. Legislation and the Skin at Work

3. The Skin as a Barrier

4. Occupational Skin Disease

5. Occupations and Occupational Skin Disease

6. Risk Assessment for Nonrespiratory Hazards

7. Exposure Control-Engineering

8. Exposure Control Through Protective Equipment

9. Selection and Use of Gloves

10. Barrier Creams-Myth or Magic Answer?

11. Skin Care

12. Cross Infection and the Skin

13. Creating an Effective Skin Management System

14. Investigating a Problem at Work

15. Technology and Skin Management

The author also has a Web site [www.enviroderm.co.uk] where this book and other resources reviewed in this indexed dermal bibliography can be purchased. 
Article ID:

Citation:

Resource type:

Educational materials:

Number of references:

Industries/occupations:

Specific process:

Chemical:

Specific chemicals:

Mixtures:

Audience:

Topics addressed:

Summary:

Article ID:

Citation:

Resource type:

Educational materials:

Number of references:

Industries/occupations:

Specific process:

Chemical:

Specific chemicals:

Mixtures:

Audience:

Topics addressed:
170

Patlewicz G, Rodford R, Walker JD. Quantitative structureactivity relationships for predicting skin and eye irritation. Environ Toxicol Chem 22:1862-69.

Journal article-review, meta-analysis

No

46

No

Professional

D Hazard identification

D.3 Characterization protocols

D.3.F QSARs-development, validation, and application

This paper reviewed quantitative structure-activity relationships (QSARs) for predicting skin and eye irritation from existing experimental data.

171

Paustenbach D, Leung HW, Rothrock JA [1999]. Health risk assessment. In: Adams RM, ed. Occupational skin disease. Philadelphia: Saunders, 291-323.

Book/monograph, chapter

No

210

Yes

Professional

C Exposure characterization

C.2 Description of factors influencing exposure conditions

C.2.B Exposure concentration 


\begin{tabular}{|c|c|c|}
\hline & C.2.C & Skin area affected \\
\hline & C.2.E & Uptake \\
\hline & C. 4 & Direct methods to measure exposure \\
\hline & C.4.A & Surfaces \\
\hline & C. 5 & Exposure modeling \\
\hline & $\mathrm{D}$ & Hazard identification \\
\hline & D.2 & $\begin{array}{l}\text { Summaries of health effects, dose-response } \\
\text { relationships }\end{array}$ \\
\hline & D.3 & Characterization protocols \\
\hline & D.3.E & $\begin{array}{l}\text { Measurement of skin permeation rates and } \\
\text { reservoir effects }\end{array}$ \\
\hline & $\mathrm{E}$ & Risk assessment \\
\hline & E.1 & Guidelines for risk assessment or analysis \\
\hline & E.1.A & Localized health effects \\
\hline & E.1.B & Systemic health effects \\
\hline & E. 2 & Example of risk assessments \\
\hline Summary: & $\begin{array}{l}\text { This } \mathrm{c} \\
\text { contri } \\
\text { of occ } \\
\text { four } \mathrm{p} \\
\text { dose-r } \\
\text { risk ch } \\
\text { expost } \\
\text { measu }\end{array}$ & $\begin{array}{l}\text { aprehensive reference by over } 40 \text { clinician- } \\
\text { tors discusses diagnosis, treatment, and prevention } \\
\text { ational skin disease. This chapter addresses the } \\
\text { ses of risk assessment: hazard identification, } \\
\text { ponse assessment, exposure assessment, and } \\
\text { acterization, as well as uptake, fate of chemicals, } \\
\text { pathways, models and modeling, exposure } \\
\text { g, sensitization, and risk reduction. }\end{array}$ \\
\hline Article ID: & 172 & \\
\hline Citation: & $\begin{array}{l}\text { Phillip } \\
\text { exposL } \\
\text { Appl }\end{array}$ & $\begin{array}{l}\text { AM, Garrod AN [2001]. Assessment of dermal } \\
\text { - empirical models and indicative distributions. } \\
\text { cup Environ Hyg 16(2):323-28. }\end{array}$ \\
\hline Resource type: & Journa & article-review, meta-analysis \\
\hline Educational materials: & No & \\
\hline Number of references: & 12 & \\
\hline Industries/occupations: & & \\
\hline Specific process: & & \\
\hline Chemical: & & \\
\hline Specific chemicals: & & \\
\hline Mixtures: & No & \\
\hline Audience: & Profes & nal \\
\hline Topics addressed: & $\mathrm{C}$ & Exposure characterization \\
\hline
\end{tabular}


Summary:

$$
\begin{array}{ll}
\text { C.5 } & \text { Exposure modeling } \\
\text { E } & \text { Risk assessment } \\
\text { E.2 } & \text { Example of risk assessments }
\end{array}
$$

This article by the U.K.'s HSE proposes an exposure assessment mechanism, the "indicative distribution approach," to use when little or no direct dermal exposure data are available. It allows one to conduct a risk assessment using a simple 12-box matrix based upon two variables: profile (narrow, medium, or wide geometric standard deviation) and deposition level (1-4 mg/minute).

Article ID:

\section{Citation:}

Resource type:

Educational materials:

Number of references:

Industries/occupations:

Specific process:

Chemical:

Specific chemicals:

Mixtures:

Audience:

Topics addressed:

Summary:

\section{3}

Poet TS [2000]. Assessing dermal absorption. Toxicol Sci 58(1):1-2.

Journal article-review, meta-analysis

No

7

No

Professional

C Exposure characterization

C.2 Description of factors influencing exposure conditions

C.2.E Uptake

This short article summarizes environmental and nonenvironmental factors that contribute to dermal absorption.

\section{Article ID:}

Citation:

\section{Resource type:}

Educational materials:

Number of references:

Industries/occupations:

Specific process:

\section{4}

Portland Cement Association (PCA) [2006]. [www.cement. org/].

Web site

Yes

Construction 


\begin{tabular}{|c|c|c|}
\hline Chemical: & \multicolumn{2}{|c|}{ Corrosives } \\
\hline Specific chemicals: & \multicolumn{2}{|c|}{ Portland cement } \\
\hline Mixtures: & \multicolumn{2}{|l|}{ No } \\
\hline Audience: & \multicolumn{2}{|c|}{ General } \\
\hline \multirow[t]{13}{*}{ Topics addressed: } & A & Overview \\
\hline & A. 1 & Occurrence of skin exposures in the workplace \\
\hline & A. 2 & $\begin{array}{l}\text { Health hazards resulting from skin exposure to } \\
\text { chemicals }\end{array}$ \\
\hline & $\mathrm{B}$ & Exposure characterization \\
\hline & B. 2 & Factors that influence exposure conditions \\
\hline & B.2.A & Exposure intensity/frequency \\
\hline & B.2.B & Exposure controls \\
\hline & $\mathrm{E}$ & Risk management \\
\hline & E. 1 & Overview of skin exposure control options \\
\hline & E.3 & "Best practices"/guidelines/recommendations \\
\hline & E.3.C & Work practice/administration controls \\
\hline & E.3.D & PPE and PPE rules \\
\hline & E.3.E & $\begin{array}{l}\text { Skin management, barrier creams, moisturizers, } \\
\text { cleansers, and rubs }\end{array}$ \\
\hline Summary: & \multicolumn{2}{|c|}{$\begin{array}{l}\text { The Portland Cement Association represents cement } \\
\text { companies in the United States and Canada. It provides, } \\
\text { among other things, research, education, and public affairs } \\
\text { programs. Resources on dermal exposure that are available } \\
\text { through their Web site include a Web page on working } \\
\text { safely with concrete, as well as the following publications: }\end{array}$} \\
\hline & \multicolumn{2}{|c|}{$\begin{array}{l}\text { - Skin Safety with Cement and Concrete (video and } \\
\text { DVD) }\end{array}$} \\
\hline & \multicolumn{2}{|c|}{$\begin{array}{l}\text { Working Safely with Concrete (brochure, } \\
\text { downloadable from their Web site) }\end{array}$} \\
\hline
\end{tabular}

Article ID: $\quad 175$

Citation:

Resource type:

Educational materials:

Number of references:

Industries/occupations:

Specific process:

Chemical:
Rietschel RL [2004]. Clues to an accurate diagnosis of contact dermatitis. Dermatol Ther 17(3):224-30.

Journal article—review, meta-analysis

No

23 
Specific chemicals:

\begin{tabular}{|c|c|c|}
\hline Mixtures: & \multicolumn{2}{|l|}{ No } \\
\hline Audience: & \multicolumn{2}{|c|}{ Professional } \\
\hline \multirow[t]{11}{*}{ Topics addressed: } & $\mathrm{B}$ & Surveillance and clinical aspects \\
\hline & B.1 & $\begin{array}{l}\text { Surveillance study reporting incidences of } \\
\text { occupational skin exposures }\end{array}$ \\
\hline & B.1.A & Skin exposure major focus \\
\hline & $\mathrm{C}$ & Exposure characterization \\
\hline & C. 4 & Direct methods to measure exposure \\
\hline & C.4.B & Skin \\
\hline & $\mathrm{D}$ & Hazard identification \\
\hline & D.1 & $\begin{array}{l}\text { Potential health effects resulting from specific } \\
\text { chemicals }\end{array}$ \\
\hline & D.1.A & Irritant contact dermatitis \\
\hline & D.1.B & Allergic contact dermatitis/sensitization \\
\hline & D. 2 & $\begin{array}{l}\text { Summaries of health effects, dose-response } \\
\text { relationships }\end{array}$ \\
\hline Summary: & \multicolumn{2}{|c|}{$\begin{array}{l}\text { This paper discusses historical, morphologic, and } \\
\text { diagnostic steps one can take to accurately diagnosis } \\
\text { contact dermatitis, both allergic and irritant. A } \\
\text { comprehensive assessment of the patient's environment is } \\
\text { needed to obtain a correct diagnosis. }\end{array}$} \\
\hline
\end{tabular}

\begin{tabular}{ll} 
Article ID: & $\mathbf{1 7 6}$ \\
\hline Citation: & $\begin{array}{l}\text { Riviere JE [2002]. Percutaneous absorption of chemical } \\
\text { mixtures relevant to the Gulf War. Raleigh, NC: North } \\
\text { Carolina State University at Raleigh, ADA409100/XAB, -163. } \\
\text { Technical publication/report }\end{array}$ \\
$\begin{array}{l}\text { Resource type: } \\
\text { Educational materials: }\end{array}$ & No \\
Number of references: & 38 \\
Industries/occupations: & U.S. Military \\
Specific process: & \\
Chemical: & Pesticides \\
Specific chemicals: & N,N-Diethyl-m-toluamide (DEET), permethrin, \\
& pyridostigmine bromide, iisopropylfluorphosphate (DFP), \\
low-level sulfur mustard (RD), DFP, JP-8 jet fuel
\end{tabular}




\section{Summary:}

Article ID:

Citation:

Educational materials:

Number of references:

Industries/occupations:

Specific process:

Chemical:

Specific chemicals:

Mixtures:

Audience:

Topics addressed:
C.2 Description of factors influencing exposure conditions

C.2.E Uptake

D Hazard identification

D.3 Characterization protocols

D.3.D Potential to cause systemic effects

D.3.E Measurement of skin permeation rates and reservoir effects

This report quantifies the dermal absorption and cutaneous toxicity of chemical mixtures associated with Gulf War Illness. The research focuses on how (14)C-permethrin, pyridostigmine bromide, diisopropylfluorphosphate (DFP), low-level sulfur mustard (RD), DFP, and JP-8 jet fuel affects exposure to N,N-Diethyl-m-toluamide (DEET). These data demonstrate an effect of systemic drugs on dermal absorption and underscore the complexity of risk assessments of complex chemical mixtures.

\section{7}

Riviere JE [2004]. Quantitating absorption of complex chemical mixtures. Raleigh NC: North Carolina State University at Raleigh, College of Veterinary Medicine, PB2005-101509/XAB, -32. Resource type: Journal articlereview, meta-analysis

No

73

corrosives, pesticides

atrazine, chlorpyrifos, methylparathion, nonylphenol, pentachlorophenal, phenol, p-nitrophenyl, fenthion, propazine, simazine, triazine

Yes

Professional

C Exposure characterization

C.2 Description of factors influencing exposure conditions

C.2.E Uptake

D Hazard identification

D.3 Characterization protocols 
Summary:

Article ID:

Citation:

Resource type:

Educational materials:

Number of references:

Industries/occupations:

Specific process:

Chemical:

Specific chemicals:

Mixtures:

Audience:

Topics addressed:

Summary:
D.3.E Measurement of skin permeation rates and reservoir effects

E Risk assessment

E.2 Example of risk assessments

Although exposure to complex mixtures of chemicals is typical, estimating exposure for risk assessment is difficult because available databases are based on single-chemical exposure. This paper presents the results of tests on chemical mixture interactions that affect percutaneous absorption to define the physical chemical characteristics of the mixture, and includes a discussion of several dermal models including QSPR and IPPSF.

178

Riviere JE, Baynes RE, Smith C [2000]. Quantitating the percutaneous absorption of mechanistically defined chemical mixtures final report 15 Nov 1997-14 Nov 2000. Raleigh NC: North Carolina State University at Raleigh, Cutaneous Pharmacology and Toxicology Center, ADA386659/XAB, -109.

Technical publication/report

No

7

Transportation/Communications/Utility, Other: Jet aircraft

Petroleum products \& lubricants

Jet fuels, Jet A, JP- 8 , JP- $8+100$, jet fuel hydrocarbons, naphthalene, dodecane, hexadecane, jet fuel performance additives, DIEGME, 8Q21, Stadis 450

Yes

Professional

C Exposure characterization

C.2 Description of factors influencing exposure conditions

C.2.E Uptake

D Hazard identification

D.3 Characterization protocols

D.3.E Measurement of skin permeation rates and reservoir effects

This report discusses the percutaneous absorption and cutaneous toxicity of jet fuels (Jet A, JP-8, JP- $8+100$ ), jet fuel hydrocarbons (naphthalene, dodecane, hexadecane), 
and performance additives (DIEGME, 8Q21, Stadis 450). The report cites seven journal articles (authored or coauthored by Jim E. Riviere) that are included in full.

The articles are:

Riviere JE, Monteiro-Riviere NA, Brooks JD, Budsaba K, Smith CE [1999]. Dermal absorption and distribution of topically dosed jet fuels Jet A, JP-8, and JP-8(100). Toxicol Appl Pharmacol 160:60-75.

Allen DG, Riviere JE, Monteiro-Riviere NA [2000]. Induction of early biomarkers of inflammation produced by keratinocytes exposed to jet fuels Jet-A, JP- 8 and JP-8(100). J Biochem Molecular Toxicol 14:231-237.

Budsaba K, Smith CE, Riviere JE [2000]. Compass plots: a combination of star plot and analysis of means (ANOM) to visualize significant interactions in complex toxicology studies. Toxicol Methods 10:313-332.

Riviere JE, Brooks JD, and Qiao GL [2000]. Methods for assessing the percutaneous absorption of volatile chemicals in isolated perfused skin: studies with chloropentafluorobenzene (CPFB) and dichlorobenzene (DCB). Toxicol Methods 10:265-281.

Baynes RE, Martin T, Craigmill AL, Riviere JE [1999]. Strategies for estimating provisional acceptable residues (PAR) for extra label drug use in livestock. Regulatory Toxicol Pharmacol 29:287-299.

Allen DG, Riviere JE, Monteiro-Riviere NA [2001]. Cytokine induction as a measure of cutaneous toxicity in primary and immortalized porcine keratinocytes exposed to jet fuels and their relation to normal human keratinocytes. Toxicol Lett 119:209-217.

Rhyne BN, Pirone JR, Monteiro-Riviere NA [2002]. The use of enzyme histochemistry in detecting cutaneous toxicity of three topically applied jet fuel mixtures. Toxicol Mechanisms and Methods 12:17-34.

Article ID: Citation:

\section{9}

Riviere JE, Monteiro-Riviere NA, Baynes RE, Xia X, Smith C [2004]. Quantitating the percutaneous absorption of mechanistically defined chemical mixtures final report 15 Dec 2000-14 Dec 2003. Raleigh NC: North Carolina State University at Raleigh, Cutaneous Pharmacology and Toxicology Center, ADA422081/XAB, -33. 
Resource type:

Educational materials:

Number of references:

Industries/occupations:

Specific process:

Chemical:

Specific chemicals:

Mixtures:

Audience:

Topics addressed:

Summary:

Technical publication/report

No

11

Transportation/Communications/Utility, Other: Jet aircraft

Petroleum products \& lubricants

Jet fuel, Jet A, JP-8, JP-8(100)

No

Professional

C Exposure characterization

C.1 Workplace factors associated with harmful skin exposures

C.2 Description of factors influencing exposure conditions

C.2.E Uptake

D Hazard identification

D.1 Potential health effects resulting from specific chemicals

D.1.C Systemic toxicity

D.2 Summaries of health effects, dose-response relationships

This report assesses the dermal absorption and skin toxicity of topically applied jet fuels and their additives using pigs, in vitro porcine skin and inert membrane models, as well as human keratinocyte cell cultures.
Article ID:

Citation:

Resource type:

Educational materials:

Number of references:

Industries/occupations:

Specific process:

Chemical:

Specific chemicals:

Mixtures:

\section{0}

Rodford R, Patlewicz G, Walker JD, Payne MP [2003]. Quantitative structure-activity relationships for predicting skin and respiratory sensitization. Environ Toxicol Chem 22(8):1855-61.

Journal article—review, meta-analysis

No

28

Solubility data provided for 15 chemicals

No 
Audience:

Topics addressed:

Summary:

Article ID:

Citation:

Resource type:

Educational materials:

Number of references:

Industries/occupations:

Specific process:

Chemical:

Specific chemicals:

Mixtures:

Audience:

Topics addressed:
Professional

D Hazard identification

D.1 Potential health effects resulting from specific chemicals

D.1.B Allergic contact dermatitis/sensitization

D.3 Characterization protocols

D.3.F QSARs—development, validation, and application

This paper reviewed quantitative structure-activity relationships (QSARs) for predicting skin and respiratory sensitization from existing experimental data.

\section{1}

Romano-Woodward D [2000]. Safe use of glutaraldehyde. Nurs Stand 14(32):47-51.

Magazine article

No

0

cleaning agents

glutaraldehyde

No

General

A Overview

A.2 Health hazards resulting from skin exposure to chemicals

E Risk management

E.2 Protocols/checklists to monitor potential exposures

E.3 "Best practices"/guidelines/recommendations

E.3.A Substitution

E.3.B Engineering controls

E.3.D PPE and PPE rules

E.4 Guidelines/recommendations for postexposure skin decontamination

This article explains what precautions should be used for handling glutaraldehyde, a chemical used in many healthcare settings to sterilize instruments. 
Article ID:

\section{Citation:}

Resource type:

Educational materials:

Number of references:

Industries/occupations:

Specific process:

Chemical:

Specific chemicals:

Mixtures:

Audience:

Topics addressed:

Summary:
182

Ross JH, Dong MH, Krieger RI [2000]. Conservatism in pesticide exposure assessment. Regul Toxicol Pharmacol 31(1):53-58.

Journal article_-review, meta-analysis

No

39

No

Professional

C Exposure characterization

C.2 Description of factors influencing exposure conditions

C.2.A Exposure intensity/frequency/duration

C.2.E Uptake

E Risk assessment

E.1 Guidelines for risk assessment or analysis

E.1.A Localized health effects

E.1.B Systemic health effects

This article discusses three exposure assessment factors that, could result in a significant overestimate of exposures to pesticides. The factors are (1) dermal absorption from animal studies, (2) daily dose extrapolated from partial day monitoring, and (3) nonbolus dosages from dermal or inhalation exposure. The authors recommend the generation of more appropriate data to minimize exposure overestimation, specifically human dermal absorption data, as well as conducting full-day exposure monitoring studies and, if feasible, generating dermal rather than oral toxicology data in those cases where the dermal route predominates.
Article ID:

\section{Citation:}

Resource type:

Educational materials:

Number of references:
183

Rowse DH, Emmett EA [2004]. Solvents and the skin. Clin Occup Environ Med 4(4):657-730, vi.

Journal article_-review, meta-analysis

No

331 


\section{Industries/occupations: \\ Specific process: \\ Chemical:}

Specific chemicals:

Mixtures:

Audience:

Topics addressed:
PAHs, solvents

Table 1 provides applications, volatility, potential routes of entry, toxicity rating, skin lesions type, and health effects for 80 specific chemicals.

Table 2 provides regulatory/guideline limits for 80 specific chemicals from the International Agency for Research on Cancer (IARC), ACGIH TWA, ACGIH short term exposure limit (STEL), ACGIH TLV, NIOSH, OSHA, and the Montreal protocol.

Describes health effects, permeability, and other information on 40 specific alcohols, aldehydes, aliphatic and alicyclic hydrocarbons, amides, amines, aromatic hydrocarbons, chlorinated hydrocarbons, esters, ethers, glycol ethers, ketones, phenols, and terpenes.

No

Professional

A Overview

A.2 Health hazards resulting from skin exposure to chemicals

A.4 Skin physiology and function as barriers to chemical insults

A.5 Dermal regulations and skin notations

C Exposure characterization

C.2 Description of factors influencing exposure conditions

C.2.E Uptake

D Hazard identification

D.1 Potential health effects resulting from specific chemicals

D.1.A Irritant contact dermatitis

D.1.B Allergic contact dermatitis/sensitization

D.1.C Systemic toxicity

D.1.D Other health effects

D.2 Summaries of health effects, dose-response relationships

D.3 Characterization protocols

D.3.E Measurement of skin permeation rates and reservoir effects

This paper examines skin structure, permeability, and chemical uptake (injuries caused by solvents). Included are 
reviews of solvent dermal health effects and the potential for systemic toxicity from dermal absorption.

Article ID:

Citation:

Resource type:

Educational materials:

Number of references:

Industries/occupations:

Specific process:

Chemical:

Specific chemicals:

Mixtures:

Audience:

Topics addressed:

Summary:

\section{4}

Roy A, Weisel CP, Lioy PJ, Georgopoulos PG [1996]. A

distributed parameter physiologically-based pharmacokinetic model for dermal and inhalation exposure to volatile organic compounds. Risk Anal 16(2):147-60.

Journal article—review, meta-analysis

No

30

VOCs

No

Professional

C Exposure characterization

C.2 Description of factors influencing exposure conditions

C.2.A Exposure intensity/frequency/duration

C.2.B Exposure concentration

C.2.C Skin area affected

C.2.E Uptake

C.5 Exposure modeling

This paper compares the way three models, developed to estimate dermal dose from exposures to toxic chemicals, estimate chloroform dose. Two are physiologically based pharmacokinetic models (PBPKs). The other is a more recently developed generalized "distributed parameter" physiologically based pharmacokinetic model (DP-PBPK), which has been developed for short-term exposures. The three models were evaluated by comparing simulated postexposure exhaled breath concentration profiles with measured concentrations following environmental chloroform exposures. All three models yielded estimates close to that of measured exhaled breath concentrations. Differences are described in detail.
Article ID:

Citation:

\section{5}

Sahmel J, Boeninger M. Dermal exposure assessments. In: Bullock W, et al., ed. A strategy for assessing and managing 
Resource type:

Educational materials:

Number of references:

Industries/occupations:

Specific process:

Chemical:

Specific chemicals:

Mixtures:

Audience:

Topics addressed: occupational exposures, 3rd ed. Fairfax, VA: AIHA Press, 137-61.

Book/monograph, chapter

No

103
No

Professional

A Overview

A.4 Skin physiology and functions as a barrier to chemical insults

C Exposure characterization

C.2 Description of factors influencing exposure conditions

C.2.A Exposure intensity/frequency/duration

C.2.B Exposure concentration

C.2.C Skin area affected

C.2.E Uptake

C.4 Direct methods to measure exposure

C.4.A Surfaces

C.4.B Skin

C.4.C Biomonitoring

C.5 Exposure modeling

E Risk assessment

E.1 Guidelines for risk assessment or analysis

E.1.A Localized health effects

E.1.B Systemic health effects

E.2 Example of risk assessments

Topics include

Barriers to conducting dermal exposure assessments Evaluating dermal exposures for local and systemic toxicity

Conducting dermal exposure assessments

Characterizing dermal exposures

Reviewing pertinent literature

Toxicology and absorption data

Dermal OELs 
SEG determination for dermal exposures

Dermal exposure assessment factors

Dermal contact area

Dermal concentration

Dermal contract frequency

Dermal retention time

Dermal penetration potential

Affects of skin health on dermal penetration

Judging of dermal exposure profiles

Uncertain dermal exposures

Dermal modeling approaches

Dermal modeling approaches

Evaluation of quantitative or semiquantitative data

Example: Using skin monitoring data for lead in metal

cutting

References

Article ID:

\section{Citation:}

Resource type:

Educational materials:

Number of references:

Industries/occupations:

Specific process:

Chemical:

Specific chemicals:

Mixtures:

Audience:

Topics addressed:

Summary:

\section{6}

Sarkis K [2000]. Protecting hands against chemical exposures. Occupational Hazards 62(8):53-56.

Magazine article

Yes

0
No

General

E Risk management

E.3 "Best practices"/guidelines/recommendations

E.3.D PPE and PPE rules

The paper discusses how gloves prevent skin exposures, as well as how to select gloves based upon the material being handled, the hazard involved, the task being performed, and comfort. It also discusses the pros and cons of latex, nitrile, neoprene, polyvinylchloride (PVC), polyvinylalcohol (PVA), butyl, and viton gloves.

Article ID:

\section{7}

Sartorelli P, Andersen HR, Angerer J, Corish J, Drexler H, Goen T, Griffin P, Hotchkiss SA, Larese F, Montomoli L, 


\section{Resource type:}

Educational materials:

Number of references:

Industries/occupations:

Specific process:

Chemical:

Specific chemicals:

Mixtures:

Audience:

Topics addressed:
Perkins J, Schmelz M, van de Sandt J, Williams F [2000]. Percutaneous penetration studies for risk assessment. Environ Toxicol Pharmacol 8(2):133-52.

Journal article—review, meta-analysis

No

77

Soaps and detergents, solvents

Dimethylsulfoxide (DMSO)

No

Professional

A Overview

A.3 Investigation, intervention, and control of occupational skin exposures

C Exposure characterization

C.2 Description of factors influencing exposure conditions

C.2.E Uptake

D Hazard identification

D.3 Characterization protocols

D.3.E Measurement of skin permeation rates and reservoir effects

E Risk assessment

E.1 Guidelines for risk assessment or analysis

E.1.A Localized health effects

This paper by the Percutaneous Penetration Subgroup of the European Community's Dermal Exposure Network presents issues related to percutaneous penetration (uptake) rates for important chemicals, factors affecting those update rates, and gaps in knowledge in the field of percutaneous penetration. Sections include

1. Introduction

2. The use of percutaneous penetration data in risk assessment

3. Factor influencing the choice of cell characteristics for percutaneous penetration in vitro studies

4. Factors influencing the choice of the donor phase for percutaneous penetration in vitro studies 
5. Factors influencing the choice of skin and membrane for percutaneous penetration in vitro studies

6. Factors influencing the choice of receptor fluids for percutaneous penetration in vitro studies

7. The presentation of in vitro percutaneous penetration results

8. Existing guidelines on percutaneous penetration in vitro studies

9. Prediction of plasma levels from penetration data

10. The influence of cutaneous metabolism on skin absorption

11. Criteria for the selection of reference compounds for in vitro percutaneous penetration

12. Correlation between in vitro and in vivo experiments

13. The use of microdialysis for the determination of dermal penetration of hazardous substances in vivo

Article ID:

\section{Citation:}

Resource type:

Educational materials:

Number of references:

Industries/occupations:

Specific process:

Chemical:

Specific chemicals:

Mixtures:

Audience:

Topics addressed:

Summary:

\section{8}

Sartorelli P [2002]. Dermal exposure assessment in occupational medicine. Occup Med (Lond) 52(3):151-56.

Journal article-review, meta-analysis

No

16

\section{No}

Professional

A Overview

A.1 Occurrence of skin exposures in the workplace

C Exposure characterization

C.2 Description of factors influencing exposure conditions

C.2.E Uptake

This paper provides a discussion of various methods for assessing dermal exposure, including threshold limit 
values, QSARs, and occupational exposure limit skin notations.

Article ID: Citation:

Resource type:

Educational materials:

Number of references:

Industries/occupations:

Specific process:

Chemical:

Specific chemicals:

Mixtures:

Audience:

Topics addressed:

Summary:
189

Schlede E, Aberer W, Fuchs T, Gerner I, Lessmann H, Maurer T, Rossbacher R, Stropp G, Wagner E, Kayser D [2003]. Chemical substances and contact allergy-244 substances ranked according to allergenic potency. Toxicology 193(3):219.

Journal article-review, meta-analysis

No

15
No

Professional

D Hazard identification

D.1 Potential health effects resulting from specific chemicals

D.1.B Allergic contact dermatitis/sensitization

D.3 Characterization protocols

D.3.C Sensitization potential

In 2001, 30 experts, including university dermatologists, industry representatives, and regulators, concluded a 15 -year project to rank 244 chemicals by contact allergenic potency based on clinical and experimental data on humans and animals. The chemicals were assigned to one of three categories. Category A (98 substances) includes potent contact allergens with significant allergenic properties. Category B (77 substances) includes substances with a solid-based indication of a contact allergenic potential and substances with the capacity of crossreactions. Category C (69 substances) includes substances with insignificant or questionable allergenic effects.
Article ID:

Citation:

\section{0}

Schneider T, Vermeulen R, Brouwer DH, Cherrie JW, Kromhout H, Fogh CL [1999]. Conceptual model for 
Resource type:

Educational materials:

Number of references:

Industries/occupations:

Specific process:

Chemical:

Specific chemicals:

Mixtures:

Audience:

Topics addressed:

Summary: assessment of dermal exposure. Occup Environ Med 56(11):765-73.

Journal article—review, meta-analysis

No

47
No

Professional

C Exposure characterization

C.2 Description of factors influencing exposure conditions

C.2.A Exposure intensity/frequency/duration

C.2.B Exposure concentration

C.2.C Skin area affected

C.2.E Uptake

C.4 Direct methods to measure exposure

C.4.B Skin

C.5 Exposure modeling

This paper presents a multicompartment model for dermal exposure assessment. The model describes the transport of a contaminant mass from the source of the hazardous substance to the surface of the skin. The model also offers a standardized method of measurement using consistent terminology. The merits of existing models are also discussed.
Article ID:

Citation:

Resource type:

Educational materials:

Number of references:

Industries/occupations:

Specific process:

Chemical:
191

Schneider T, Cherrie JW, Vermeulen R, Kromhout H [2000]. Dermal exposure assessment. Ann Occup Hyg 44(7):493-99.

Journal article—review, meta-analysis

No

35 
Specific chemicals:

Mixtures:

Audience:

Topics addressed:

Summary:
No

Professional

C Exposure characterization

C.4 Direct methods to measure exposure

C.4.A Surfaces

C.4.B Skin

C.4.C Biomonitoring

C.5 Exposure modeling

The authors propose a theoretical strategy to assess dermal exposure based on a conceptual model for airborne contaminants. Many different skin and surface measurements are evaluated.
Article ID:

Citation:

Resource type:

Educational materials:

Number of references:

Industries/occupations:

Specific process:

Chemical:

Specific chemicals:

Mixtures:

Audience:

Topics addressed:

\section{2}

Schnuch A, Lessmann H, Schulz KH, Becker D, Diepgen TL, Drexler H, Erdmann S, Fartasch M, Greim H, KrickeHelling P, Merget R, Merk H, Nowak D, Rothe A, Stropp G, Uter W, Wallenstein G [2002]. When should a substance be designated as sensitizing for the skin ('Sh') or for the airways ('Sa')? Hum Exp Toxicol 21(8):439-44.

Journal article—review, meta-analysis

No

10
No

Professional

A Overview

A.5 Dermal regulations and skin notations

D Hazard identification

D.1 Potential health effects resulting from specific chemicals

D.1.B Allergic contact dermatitis/sensitization

The article reviews the criteria for determining when a substance should be deemed an airway sensitizer ("Sa") or skin sensitizer ("Sh") according to the list of maximum allowable concentration (MAK) and biological tolerance 
(BAT) values published annually by the Commission of the Deutsche Forschungsgemeinschaft for the Investigation of Health Hazards of Chemical Compounds in the Work Area (MAK Commission). The authors conclude that MAK and BAT values make the classification of substances more rational, consistent, comprehensible, and transparent, but their application may not be necessary or possible in some cases.

Article ID:

\section{Citation:}

Resource type:

Educational materials:

Number of references:

Industries/occupations:

Specific process:

Chemical:

Specific chemicals:

Mixtures:

Audience:

Topics addressed:

Summary:

\section{3}

Schuhmacher-Wolz U, Kalberlah F, Oppl R, van

Hemmen JJ [2003]. A toolkit for dermal risk assessment: Toxicological approach for hazard characterization. Ann Occup Hyg 47(8):641-52.

Journal article—review, meta-analysis

No

34

\section{No}

Professional

E Risk assessment

E.1 Guidelines for risk assessment or analysis

E.1.A Localized health effects

E.1.B Systemic health effects

This article is the 6th article of a 6-part series on RISKOFDERM, a tool for conducting risk assessments. The series was published in the Annals of Occupational Hygiene in 2003. The following briefly summarizes each paper in the series:

1. ID 212-Outlines a "toolkit" for conducting dermal occupational risk assessment.

2. ID 163-Describes the assumptions in the toolkit and describes an approach to exposure assessment used by the toolkit.

3. ID 139-Describes the determinants relevant for dermal exposure models in the scope of regulatory risk assessment.

4. ID 219-Describes how default dermal exposure values can be adjusted for specific work situations. 
5. ID 100-Describes the derivation of the toolkit's default task-based dermal exposure values.

6. ID 193-Describes the development of "intrinsic toxicity" (IT) scores used for hazard characterization.

Article ID:

194

Citation:

Resource type:

Educational materials:

Number of references:

Industries/occupations:

Specific process:

Chemical:

Specific chemicals:

Mixtures:

Audience:

Topics addressed:
Semple S [2004]. Dermal exposure to chemicals in the workplace: Just how important is skin absorption? Occup Environ Med 61(4):376-82.

Journal article-review, meta-analysis

No

31

General-overview

General-overview, solvents

No

Professional

A Overview

A.1 Occurrence of skin exposures in the workplace

A.2 Health hazards resulting from skin exposure to chemicals

A.4 Skin physiology and functions as a barrier to chemical insults

C Exposure characterization

C.2 Description of factors influencing exposure conditions

C.2.A Exposure intensity/frequency/duration

C.2.B Exposure concentration

C.2.C Skin area affected

C.2.E Uptake

C.4 Direct methods to measure exposure

C.4.B Skin

C.4.C Biomonitoring

C.5 Exposure modeling

This paper discusses the importance of occupational dermal exposure, factors that influence exposure and absorption, and methods for measuring and assessing dermal exposure. 
Article ID:

Citation:

Resource type:

Educational materials:

Number of references:

Industries/occupations:

Specific process:

Chemical:

Specific chemicals:

Mixtures:

Audience:

Topics addressed:

Summary:
195

Semple S, Brouwer DH, Dick F, Cherrie JW [2001]. A

dermal model for spray painters, Part II: Estimating

the deposition and uptake of solvents. Ann Occup Hyg

45(1):25-33.

Journal article-primary

No

26

Construction

Spray painting

No

Professional

C Exposure characterization

C.1 Workplace factors associated with harmful skin exposures

C.2 Description of factors influencing exposure conditions

C.2.A Exposure intensity/frequency/duration

C.2.B Exposure concentration

C.2.C Skin area affected

C.2.E Uptake

Part 2 of 2. This paper presents a model based upon "a process-based, structured approach" that both estimates occupational dermal exposure and uptake of solvents, using airless spray painters as an example. Estimates are based upon spray technique, object shape, workers' individual work practices, and droplet formation and deposition. Predicted exposure showed reasonable correlation with the actual measured exposure and the authors conclude that a structured, process-based approach has the potential to produce reliable estimates of dermal exposure. The authors also call for additional field studies.

Part 2 identifies the determinants of exposure, calculates the flux of solvent through the stratum corneum, and estimates total dermal uptake using a range of exposure scenarios.
Article ID:

Citation:
196

Shum KW, Meyer JD, Chen Y, Cherry N, Gawkrodger

DJ [2003]. Occupational contact dermatitis to nickel: 
Resource type:

Educational materials:

Number of references:

Industries/occupations:

Specific process:

\section{Chemical:}

Specific chemicals:

Mixtures:

Audience:

Topics addressed:

Summary: experience of the British dermatologists (EPIDERM) and occupational physicians (OPRA) surveillance schemes. Occup Environ Med 60(12):954-57.

Journal article—primary

No

31

Beauty/Cosmetology, Service-Medical

The study focused on the following occupational categories:

Hairdressers, Bar staff, Chefs/cooks, Retail cash and checkout operators, Catering assistants, Counter clerks/ cashiers, Cleaners, Nurses, Metal workers, Sales assistants, Secretaries

Heavy metals/inorganic compounds

Nickel

No

Professional

B Surveillance and clinical aspects

B.1 Surveillance study reporting incidences of occupational skin exposures

\section{B.1.A Skin exposure major focus}

This study used occupational surveillance reporting databases (EPIDERM and OPRA) to determine to what extent nickel caused occupational contact dermatitis in the U.K. The study concluded that up to $12 \%$ of total estimated cases of occupational contact dermatitis were due in part to nickel exposure.

197

Article ID:

Smith Pease CK [2003]. From xenobiotic chemistry and metabolism to better prediction and risk assessment of skin allergy. Toxicology 192(1):1-22.

Resource type:

Journal article—review, meta-analysis

Educational materials:

No

Number of references:

63

Industries/occupations:

Specific process:

Chemical:

Specific chemicals:

Mixtures: 
Audience:

Topics addressed:

Summary:
Professional

A Overview

A.4 Skin physiology and function as barriers to chemical insults

C Exposure characterization

C.2 Description of factors influencing exposure conditions

C.2.E Uptake

D Hazard identification

D.1 Potential health effects resulting from specific chemicals

D.1.B Allergic contact dermatitis/sensitization

D.2 Summaries of health effects, dose-response relationships

D.3 Characterization protocols

D.3.C Sensitization potential

D.3.F QSARs—development, validation, and application

This review explores general chemical and metabolic processes involved in the process of skin sensitization to chemicals. It also discusses recent work using xenobiotics to explore sensitization mechanisms.
Article ID:

Citation:

Resource type:

Educational materials:

Number of references:

Industries/occupations:

Specific process:

Chemical:

Specific chemicals:

Mixtures:

Audience:

Topics addressed:

\section{8}

Soutar A, Semple S, Aitken RJ, Robertson A [2000]. Use of patches and whole body sampling for the assessment of dermal exposure. Ann Occup Hyg 44(7):511-18.

Journal article—review, meta-analysis

No

37

No

Professional

C Exposure characterization

C.4 Direct methods to measure exposure

C.4.B Skin 
C.5 Exposure modeling

Summary:
This paper details the principles underlying patch and whole body sampling and their advantages and disadvantages. This paper takes a recent conceptual model for dermal exposure and discusses the role that the various techniques may play in the application of this model.

199

Article ID:

Susitaival P, Flyvholm MA, Meding B, Kanerva L, Lindberg M, Svensson A, Olafsson JH [2003]. Nordic Occupational Skin Questionnaire (NOSQ-2002): a new tool for surveying occupational skin diseases and exposure. Contact Dermatitis 49(2):70-76

Resource type: Journal article-review, meta-analysis

Educational materials: No

Number of references: 46

Industries/occupations:

Specific process:

Chemical:

Specific chemicals:

Mixtures:

No

Audience:

Topics addressed:

Summary:

Article ID:

\section{0}

Citation:

Syracuse Research Corporation (SRC) [2006]. SRC

business areas: Environmental science. [www.syrres.com/ esc/default1.htm]. Date accessed: April 14, 2008.

Resource type:

Data file

Educational materials:

No

Number of references:

Industries/occupations:

General-overview

Specific process: 
Chemical:

Specific chemicals:

Mixtures:

Audience:

Topics addressed:

Summary:
Abrasives, cleaning agents, coolants, corrosives, fiberglass and other fibers, heavy metals/inorganic compounds, organic dyes, particulates, pesticides, petroleum products \& lubricants, plastics and resins, PAHs, PCBs, solvents

No

Professional

C Exposure characterization

C.2 Description of factors influencing exposure conditions

C.2.E Uptake

C.4 Direct methods to measure exposure

C.4.B Skin

D Hazard identification

D.1 Potential health effects resulting from specific chemicals

D.1.A Irritant contact dermatitis

D.1.B Allergic contact dermatitis/sensitization

D.3 Characterization protocols

D.3.D Potential to cause systemic effects

D.3.E Measurement of skin permeation rates and reservoir effects

SRC is a not-for-profit, independent, research and development organization. SRC's environmental science area has expertise in analyzing information on occupational and environmental hazards. SRC has made available a number of different software programs that relate to skin exposures to chemicals including:

- DermWin, which estimates the dermal permeability coefficient (Kp) used to estimate the potential for a chemical to be absorbed through the skin.

- KowWin, which estimates the log octanol-water partition coefficient, $\log$ P, of chemicals using an atom/fragment contribution method developed at SRC.

- WsKow, which estimates an octanol/water partition coefficient using the algorithms in SRC's LogKow Program and estimates a chemical's water solubility from this value.

SRC also developed the Toxic Substances Control Act Test Submission Database (TSCATS), which is used for the collection, maintenance, and dissemination of information 
on unpublished technical reports submitted by industry to the USEPA under TSCA.

Article ID:

Citation:

Resource type:

Educational materials:

Number of references:

Industries/occupations:

Specific process:

Chemical:

Specific chemicals:

Mixtures:

Audience:

Topics addressed:

Summary:

\section{1}

ten Berge W [2004]. Home page of Wil ten Berge: Wil ten Berge model for dermal absorption. [http://home.planet. $\mathrm{nl} / \sim$ wtberge/].

Web site

No

No

Professional

C Exposure characterization

C.5 Exposure modeling

D Hazard identification

D.3 Characterization protocols

D.3.F QSARs-development, validation, and application

This home page of Wil ten Berge contains a downloadable version of the SKINDERM Program, which can to used for the estimation of the skin permeation coefficients of aqueous and vapor chemicals using physico-chemical properties of the chemical and the octanol/water partition coefficient. This program is based on a QSAR database developed by A. Wilschut, W.F. ten Berge, P.J. Robinson, and T.E. McKone in 1995. The program currently contains data for over 60 chemicals.

\section{2}

Citation:

Thrall KD, Poet TS, Corley RA, Tanojo H, Edwards JA, Weitz KK, Hui X, Maibach HI, Wester RC [2000]. A real-time in-vivo method for studying the percutaneous absorption of volatile chemicals. Int J Occup Environ Health 6(2):96.

Resource type:

Journal article_-primary

Educational materials:
No 
Number of references:

Industries/occupations:

Specific process:

Chemical:

Specific chemicals:

Mixtures:

Audience:

Topics addressed:

Summary:

Article ID:

Citation:

Resource type:

Educational materials:

Number of references:
24

Solvents

Methyl chloroform, TCE, benzene

No

Professional

C Exposure characterization

C.2 Description of factors influencing exposure conditions

C.2.E Uptake

C.4 Direct methods to measure exposure

C.4.B Skin

C.5 Exposure modeling

D Hazard identification

D.2 Summaries of health effects, dose-response relationships

D.3 Characterization protocols

D.3.E Measurement of skin permeation rates and reservoir effects

This paper presents estimates of percutaneous absorption of volatile chemicals. Dermal uptake of solvents under nonsteady-state conditions was determined using realtime breath analysis in rats, monkeys, and humans.

Physiologically based pharmacokinetic (PBPK) models were used to estimate dermal permeability. The effects of the exposure matrix, occlusion versus nonocclusion, and species differences were compared for methyl chloroform, TCE, and benzene. The method was found to be sufficiently sensitive for animal and human dermal studies at low exposure concentrations over small body surface areas for short periods and using nonsteady-state exposure conditions.
Toeppen-Sprigg B [1999]. Management of dermatitis in the rubber manufacturing industry. Occup Med 14(4):797-818.

Journal article—review, meta-analysis

No

51 
Industries/occupations:

Specific process:

Chemical:

Specific chemicals:

Mixtures:

Audience:

Topics addressed:
Summary:

Article ID:

Citation:

Resource type:

Educational materials:

Number of references:
Manufacturing—Rubber manufacturing

Latex, rubber additives

No

Professional

A Overview

A.1 Occurrence of skin exposures in the workplace

A.2 Health hazards resulting from skin exposure to chemicals

A.3 Investigation, intervention, and control of occupational skin exposures

B Surveillance and clinical aspects

B.1 Surveillance study reporting incidences of occupational skin exposures

B.1.A Skin exposure major focus

F Risk management

F.1 Exposure control strategies

F.1.A Substitution

F.1.D PPE and PPE rules

F.1.E Skin management, barrier creams, moisturizers, cleansers, and rubs

F.2 Protocols for risk management

F.2.B Development of approach to achieve exposure reduction goal

This article examines dermatitis in the rubber industry. Contributing agents include both natural rubber and the various additives used in its manufacture. The paper reviews prevention and control measures such as substitution, PPE, barriers creams, and monitoring. It also includes a discussion of the diagnosis and treatment of dermatitis.
Tupker RA [2003]. Prediction of irritancy in the human skin irritancy model and occupational setting. Contact Dermatitis 49(2):61-69.

Journal article—review, meta-analysis

No

64 
Industries/occupations:

Specific process:

Chemical:

Specific chemicals:

Mixtures:

No

Audience:

Professional

Topics addressed:

A Overview

A.4 Skin physiology and function as barriers to chemical insults

C Exposure characterization

C.2 Description of factors influencing exposure conditions

C.2.A Exposure intensity/frequency/duration

C.2.B Exposure concentration

C.4 Direct methods to measure exposure

C.4.B Skin

Summary:

This review presents findings in the field of skin irritancy testing and discusses factors that determine irritancy testing outcome, including extrinsic and intrinsic factors such as prior exposure and genetics. This review also discusses the results from prospective cohort studies as they relate to factors influencing the development of occupational dermatitis.

Article ID: 205

Citation:

Resource type:

Educational materials:

Number of references:

Industries/occupations:

Specific process:

Chemical:

Specific chemicals:

Mixtures:

Audience:

Topics addressed:
Unison [2005]. Health and safety zone. [www.unison.org. uk/safety/index.asp].

Web page

No

General—overview

General-overview

No

General

A Overview

A.1 Occurrence of skin exposures in the workplace 
A.2 Health hazards resulting from skin exposure to chemicals

B Exposure characterization

B.1 Job/tasks, industries/processes, or chemicals associated with skin exposures

E Risk management

E.1 Overview of skin exposure control options

Summary:

UNISON is Great Britain's biggest trade union, with members working in the essential utilities and for private contractors providing public services. Their Web site includes a health and safety zone, which contains information on different health and safety topics. Included is an information sheet on dermatitis, which provides background information on causes of dermatitis in the workplace and steps that can be taken to prevent dermatitis. The information provided on legal requirements is specific to Great Britain.

Article ID:

Citation:

Resource type:

Educational materials:

Number of references:

Industries/occupations:

Specific process:

Chemical:

Specific chemicals:

Mixtures:

Audience:

Topics addressed:

\section{6}

Oregon State University [2006]. University of CaliforniaDavis, Oregon State University, Michigan State University, Cornell University, and University of Idaho. The EXTension TOXicology NETwork (EXTOXNT). [http://extoxnet.orst.edu/ghindex.html].

Web site

No

Agricultural

Pesticides

No

Professional

A Overview

A.2 Health hazards resulting from skin exposure to chemicals

A.5 Dermal regulations and skin notations

D Hazard identification

D.1 Potential health effects resulting from specific chemicals

D.1.A Irritant contact dermatitis 
Summary:

Article ID:

Citation:

Resource type:

Educational materials:

Number of references:

Industries/occupations:

Specific process:

Chemical:

Specific chemicals:

Mixtures:

Audience:

Topics addressed:
D.1.B Allergic contact dermatitis/sensitization

D.1.C Systemic toxicity

D.2 Summaries of health effects, dose-response relationships

EXTOXNET is a cooperative effort of University of California-Davis, Oregon State University, Michigan State University, Cornell University, and the University of Idaho. Their Web site contains resources about exposure to toxicants in our environment. The Pesticide Information Profiles contain basic toxicology information about pesticides, including, where relevant, information on skin exposure and toxicological effects.

\section{7}

U.S. Army Center for Health Promotion and Preventive Medicine (USACHPPM) [2006] [http://chppm-www. apgea.army.mil/].

Web site

Yes

Military

Pesticides, petroleum products \& lubricants, solvents

JP-8 jet fuel, paints

No

General

A Overview

A.1 Occurrence of skin exposures in the workplace

B Exposure characterization

B.1 Job/tasks, industries/processes, or chemicals associated with skin exposures

B.3 Protocols/checklists to characterize exposure to skin hazards

E Risk management

E.3 "Best practices"/guidelines/recommendations

E.3.C Work practice/administration controls

E.3.D PPE and PPE rules

E.3.E Skin management, barrier creams, moisturizers, cleansers, and rubs 
Summary:

Article ID:

Citation:

Resource type:

Educational materials:

Number of references:

Industries/occupations:

Specific process:

Chemical:

Specific chemicals:

Mixtures:

Audience:

Topics addressed:

Summary:
E.4 Guidelines/recommendations for postexposure skin decontamination

The U.S. Army Center for Health Promotion and Preventive Medicine's Web site has a variety of resources available about identifying, assessing and controlling environmental, occupational, and disease threats in support of the national military. Their Web site has Post Deployment Exposure Fact Sheets that address exposure, including dermal, to chemicals, such as paints, solvents, pesticides, and jet fuel. The fact sheets include information on personal protective equipment and control measures, signs and symptoms of acute and chronic exposure, reversibility of acute and chronic health effects, treatment options, and long term surveillance requirements.

\section{8}

United States Department of Transportation (USDOT)

[2004]. Emergency response guidebook. [http://hazmat. dot.gov/pubs/erg/gydebook.htm].

Web page

No

General-overview

General-overview, abrasives, cleaning agents, coolants, corrosives, fiberglass and other fibers, heavy metals/ inorganic compounds, organic dyes, particulates, pesticides, petroleum products \& lubricants, plastics and resins, PAHs, PCBs, rubber additives, solvents

Covers hundreds of different chemicals

No

Professional

F Risk management

F.1 Exposure control strategies

F.1.A Substitution

F.1.B Engineering controls

F.1.C Work practice/Administrative controls

F.1.D PPE and PPE rules

The ERG (2004) was developed jointly by the USDOT, Transport Canada, and the Secretariat of Communications and Transportation of Mexico (SCT) for use by firefighters, police, and other emergency services personnel who may be the first to arrive at the scene of a transportation 
incident involving a hazardous material. The ERG was designed as a guide to aid first responders in (1) quickly identifying the specific or generic classification of the material(s) involved in the incident and (2) protecting themselves and the general public during this initial response phase of the incident. The ERG is updated every three to four years.

Each chemical or material listed in the guide book is assigned a corresponding response guide number. The guides are then used to direct first responders on how to safely respond to hazardous material incidents. Information provided in the guides includes general health hazards including any associated dermal hazards, recommended personal protective equipment, and proper emergency response procedures. The guide can be searched by material name or identification number. The guidebook is available online as a searchable database as well as in hard copy form.

Article ID:

209

Citation:

Resource type:

Educational materials:

Number of references:

Industries/occupations:

Specific process:

Chemical:

Specific chemicals:

Mixtures:

Audience:

Topics addressed:

Summary:
USEPA [2005]. USEPA, OPPT: Exposure assessment tools and models. [www.epa.gov/opptintr/exposure/index.htm].

Web page

No

General—overview

General-overview

No

Professional

C Exposure characterization

C.5 Exposure modeling

E Risk assessment

E.1 Guidelines for risk assessment or analysis

E.1.A Localized health effects

E.1.B Systemic health effects

E.2 Example of risk assessments

The USEPA OPPT has developed several exposure assessment methods, databases, and predictive models to help in evaluating, among other things, how workers may be exposed to chemicals. This Web page provides a table of the USEPA 
exposure assessment tools and models, whether they include a dermal component, whether they address workplace exposures, and links to where they can be downloaded. Methods listed include exposure assessment screening tools.

Article ID:

\section{Citation:}

Resource type:

Educational materials:

Number of references:

Industries/occupations:

Specific process:

Chemical:

Specific chemicals:

Mixtures:

Audience:

Topics addressed:
210

van de Sandt J, Johannes JM, Dellarco M, van Hemmen

J. From dermal exposure to internal dose. J Exposure Sci Environ Epidemiol 17:S38-S47.

Journal article—review, meta-analysis

No
No

Professional

A Overview

A.4 Skin physiology and functions as a barrier to chemical insults

C Exposure characterization

C.4 Direct methods to measure exposure

C.4.A Surfaces

C.4.B Skin

C.4.C Biomonitoring

C.5 Exposure modeling

D Hazard identification

D.3 Characterization protocols

D.3.E Measurement of skin permeation rates and reservoir effects

D.3.F QSARs—development, validation, and application

E Risk assessment

E.2 Example of risk assessments

This review article discusses risk assessment, methodologies to measure dermal exposure, bioavailability data, exposure conditions, and nontesting methods for skin absorption, such as QSAR. 
Article ID:

\section{Citation:}

Resource type:

Educational materials:

Number of references:

Industries/occupations:

Specific process:

Chemical:

Specific chemicals:

Mixtures:

Audience:

Topics addressed:

Summary: van Hemmen JJ, Brouwer DH. Assessment of dermal exposure to chemicals. Sci Total Environ 168(2):131-41.

Journal article—review, meta-analysis

No

51

No

Professional

C Exposure characterization

C.2 Description of factors influencing exposure conditions

C.2.E Uptake

C.4 Direct methods to measure exposure

C.4.A Surfaces

C.4.B Skin

C.4.C Biomonitoring

This paper compares qualitative, semiqualitative, and quantitative methods for assessing dermal exposure to chemicals. These methods include job (activity) exposure profiles, surrogate skin techniques, removal techniques, tracer techniques, biological monitoring, and surface sampling techniques. The paper compares the methods by validation tests, key input factors sampling area, and collection.

Article ID:

\section{2}

Citation:

van Hemmen J, Auffarth J, Evans PG, Rajan-

Sithamparanadarajah B, Marquart H, Oppl R [2003].

RISKOFDERM: risk assessment of occupational dermal exposure to chemicals. An introduction to a series of papers on the development of a toolkit. Ann Occup Hyg 47(8):595-98.

Resource type:

Journal article—review, meta-analysis

Educational materials:

No

Number of references:

9

Industries/occupations: 
Specific process:

Chemical:

Specific chemicals:

Mixtures:

Audience:

Topics addressed:

Summary:
No

Professional

E Risk assessment

E.1 Guidelines for risk assessment or analysis

E.1.A Localized health effects

E.1.B Systemic health effects

This article is the 1st article of a 6-part series on RISKOFDERM, a tool for conducting risk assessments. The series was published in the Annals of Occupational Hygiene in 2003. The following briefly summarizes each paper in the series:

1. ID 212-Outlines a "toolkit" for conducting dermal occupational risk assessment.

2. ID 163-Describes the assumptions in the toolkit and describes an approach to exposure assessment used by the toolkit.

3. ID 139-Describes the determinants relevant for dermal exposure models in the scope of regulatory risk assessment.

4. ID 219-Describes how default dermal exposure values can be adjusted for specific work situations.

5. ID 100-Describes the derivation of the toolkit's default task-based dermal exposure values.

6. ID 193-Describes the development of "intrinsic toxicity" (IT) scores used for hazard characterization.
Article ID:

Citation:

Resource type:

Educational materials:

Number of references:

Industries/occupations:

Specific process:

Chemical:

Specific chemicals:
213

van Rooij JGM, Jongeneelen FJ [2007]. Review of skin permeation hazard of bitumen fumes. J Occup Environ Hyg 4(S1):237-244.

Journal article—review, meta-analysis

No

38

Construction

Asphalt roofing, paving

Petroleum products \& lubricants, PAHs

Bitumen fumes 
Mixtures:

Audience:

Topics addressed:

Summary:
No

Professional

A Overview

A.1 Occurrence of skin exposures in the workplace

A.2 Health hazards resulting from skin exposure to chemicals

A.3 Investigation, intervention, and control of occupational skin exposures

A.4 Skin physiology and functions as a barrier to chemical insults

C Exposure characterization

C.2 Description of factors influencing exposure conditions

C.2.E Uptake

D Hazard identification

D.1 Potential health effects resulting from specific chemicals

D.1.D Other health effects

This study presents a summary of the literature regarding the skin permeation hazard of bitumen fumes among workers.

\section{4}

\section{Citation:}

van-Wendel-de-Joode B, Brouwer DH, Vermeulen R, van

Hemmen JJ, Heederik D, Kromhout H [2003]. DREAM: A method for semi-quantitative dermal exposure assessment. Ann Occup Hyg 47(1):71-87.

\section{Resource type:}

Educational materials: Journal article—primary

Number of references:

$$
\text { No }
$$

42

Industries/occupations:

Manufacturing-Other

\section{Specific process:}

Chemical:

Specific chemicals:

Mixtures:

Audience:

Topics addressed:

Summary:
No

Professional

C Exposure characterization

C.5 Exposure modeling

This paper describes the DeRmal Exposure AssessMent (DREAM) Model for accessing and evaluating occupational 
dermal exposure to chemical and biological agents. DREAM provides an initial assessment of dermal exposure levels to liquids and solids, a framework for measuring strategies, and a basis for implementing control strategies. Two examples from the car construction industry are discussed in detail.

Article ID:

Citation:

Resource type:

Educational materials:

Number of references:

Industries/occupations:

Specific process:

Chemical:

Specific chemicals:

Mixtures:

Audience:

Topics addressed:

\section{5}

van-Wendel-de-Joode B, van Hemmen JJ, Meijster T, Major V, London L, Kromhout H [2005]. Reliability of a semi-quantitative method for dermal exposure assessment (DREAM). J Expo Anal Environ Epidemiol 15(1):111-20.

Journal article—primary

No

21

Agricultural, Manufacturing-Chemical, Transportation/ Communications/Utility

Provides dermal risk calculations for 35 industrial and agricultural tasks.

No

Professional

C Exposure characterization

C.4 Direct methods to measure exposure

C.4.A Surfaces

C.4.B Skin

C.5 Exposure modeling

The reliability of DREAM, a semiquantitative dermal exposure assessment method, was assessed by using 29 observers (mainly occupational hygienists) who were asked to fill in DREAM while performing side-by-side observations for different tasks comprising dermal exposures to liquids, solids, and vapors. The authors concluded that DREAM is useful for estimating dermal exposure both for epidemiological research and for occupational hygiene practice.
Article ID:

Citation:

\section{6}

Vermeulen R, Stewart P, Kromhout H [2002]. Dermal exposure assessment in occupational epidemiologic research. Scand J Work Environ Health 28(6):371-85. 
Resource type:

Educational materials:

Number of references:

Industries/occupations:

Specific process:

Chemical:

Specific chemicals:

Mixtures:

Audience:

Topics addressed:

Summary:
Journal article—review, meta-analysis

No

110
No

Professional

C Exposure characterization

C.2 Description of factors influencing exposure conditions

C.2.A Exposure intensity/frequency/duration

C.2.B Exposure concentration

C.2.C Skin area affected

C.2.E Uptake

C.5 Exposure modeling

This paper presents the results of a literature survey conducted to identify dermal exposure assessment methods. Variables discussed include intensity, frequency, and duration of exposure; the exposed surface area; and personal, temporal, and spatial variability in dermal exposure and uptake. Methods include qualitative, quantitative, and semiquantitative techniques. The paper focuses on dermal exposure assessment in relation to systemic effects, but local effects are also considered.
Article ID:

Citation:

Resource type:

Educational materials:

Number of references:

Industries/occupations:

Specific process:

Chemical:

Specific chemicals:

Mixtures:
217

Walker JD, Rodford R, Patlewicz G [2003]. Quantitative structure-activity relationships for predicting percutaneous absorption rates. Environ Toxicol Chem 22(8):1870-84.

Journal article—review, meta-analysis

No

50

No 
Audience:

Topics addressed:

Summary:
Professional

D Hazard identification

D.3 Characterization protocols

D.3.F QSAR—development, validation, and application

This article reviews quantitative structure-activity relationships (QSAR) for predicting percutaneous absorption rates from existing experimental data. It also provide estimates on the number of workers exposed to 25 specific chemicals and permeability coefficients $(\mathrm{Kp})$ for 83 chemicals.
Article ID:

\section{Citation:}

\section{Resource type:}

Educational materials:

Number of references:

Industries/occupations:

Specific process:

Chemical:

Specific chemicals:

Mixtures:

Audience:

Topics addressed:
218

Wang RGM, Maibach H, Knaak B, eds. [1993]. Health risk assessment: Dermal and inhalation exposure and absorption of toxicants. Boca Raton, FL: CRC Press.

Book/monograph, whole

No

1718

General—overview

General—overview, solvents

No

Professional

A Overview

A.4 Skin physiology and function as barriers to chemical insults

C Exposure characterization

C.2 Description of factors influencing exposure conditions

C.2.E Uptake

C.5 Exposure modeling

D Hazard identification

D.1 Potential health effects resulting from specific chemicals

D.1.D Other health effects

D.2 Summaries of health effects, dose-response relationships 
Summary:

Physiologically Based Pharmacokinetic Modeling to Predict Tissue Dose and Cholinesterase Inhibition in Workers Exposed to Organophosphorus and Carbamate Pesticides

Ch. 2 The Application of Pharmacokinetic Models to Predict Target Dose

Ch. 3 Cytochrome P450-Dependent Metabolism of Drugs and Carcinogens in Skin

Ch. 4 Percutaneous Absorption

Ch. 5 In Vitro Skin Metabolism

Ch. 6 Animal Models for Percutaneous Absorption

Ch. 7 A Comparative Study of the Kinetics and Bioavailability of Pure and Soil-Absorbed Benzene, Toluene, and m-Xylene After Dermal Exposure

Ch. 8 Prediction of Human Percutaneous Absorption with Physicochemical Data

Ch. 9 Dermal Absorption of TCDD: Effect of Age

Ch. 10 Percutaneous Absorption of Chemicals From Water During Swimming and Bathing

Ch. 11 Percutaneous Absorption of Contaminants From Soil

Ch. 12 General Overview of Toxicological Responses and Routes of Chemical Exposure

Ch. 13 Acute Toxicity Testing by the Dermal Route

Ch. 14 Subchronic Dermal Exposure Studies With Industrial Chemicals

Ch. 15 The Dose Response of Percutaneous Absorption 
Ch. 16 Reproductive and Developmental Toxicity Studies by Cutaneous Administration

Ch. 17 Dermal Carcinogenicity Studies of PetroleumDerived Materials

Ch. 18 Comparison of Results from Carcinogenicity Tests of Two Halogenated Compounds by Oral, Dermal and Inhalation Routes

Ch. 19 The Objectives and Goals of Dermal Carcinogenicity Testing of Petroleum Liquids

Ch. 20 Chemical Carcinogenesis in Skin: Causation, Mechanism, and Role of Oncogenes

Ch. 21 Incorporating Biological Information into the Assessment of Cancer Risk to Humans Under Various Exposure Conditions and Issues Related to High Background Tumor Incidence Rates

Ch. 22 Phototoxicity of Topical and Systemic Agents

Ch. 23 Techniques for Assessing the Health Risks of Dermal Contact with Chemicals in the Environment

Ch. 24 Interspecies Extrapolation of Toxicological Data

Ch. 25 Human Skin Xenografts to Athymic Rodents as a System to Study Toxins Delivered to or Through the Skin

Ch. 26 The Isolated Perfused Porcine Skin Flap

Ch. 27 Perspectives on Assessment of Risk from Dermal Exposure to Polycyclic Aromatic Hydrocarbons

Ch. 28 The Paradox of Herbicide 2,4,-D Epidemiology

Ch. 29 A Review of Epidemiologic Studies with Regard to Routes of Exposure to Toxicant

Article ID:

Citation:

Resource type:

Educational materials:

Number of references:
219

Warren N, Goede HA, Tijssen SC, Oppl R, Schipper HJ, van Hemmen JJ [2003]. Deriving default dermal exposure values for use in a risk assessment toolkit for small and medium-sized enterprises. Ann Occup Hyg 47(8):619-27. Journal article—review, meta-analysis

No

30 
Industries/occupations:

Specific process:

Chemical:

Specific chemicals:

Mixtures:

Audience:

Topics addressed:

Summary:

Article ID:

Citation:

Resource type:

Educational materials:

Number of references:
General—overview

General—overview

No

Professional

C Exposure characterization

C.5 Exposure modeling

E Risk assessment

E.1 Guidelines for risk assessment or analysis

E.1.A Localized health effects

E.1.B Systemic health effects

This article is the 5th article of a 6-part series on RISKOFDERM, a tool for conducting risk assessments. The series was published in the Annals of Occupational Hygiene in 2003. The following briefly summarizes each paper in the series:

1. ID 212-Outlines a "toolkit" for conducting dermal occupational risk assessment.

2. ID 163-Describes the assumptions in the toolkit and describes an approach to exposure assessment used by the toolkit.

3. ID 139-Describes the determinants relevant for dermal exposure models in the scope of regulatory risk assessment.

4. ID 219-Describes how default dermal exposure values can be adjusted for specific work situations.

5. ID 100-Describes the derivation of the toolkit's default task-based dermal exposure values.

6. ID 193-Describes the development of "intrinsic toxicity" (IT) scores used for hazard characterization.
Washington Department of Labor and Industry (WADLI). [2005]. Dermatitis: Safety and health assessment and research for prevention (SHARP). [www.lni.wa.gov/Safety/ Research/OccHealth/Derm/default.asp\#Resources].

Web page

Yes 
Industries/occupations:

Specific process:

Chemical:

Specific chemicals:

Mixtures:

Audience:

Topics addressed:

\section{Summary:}

General—overview, Agricultural,

Manufacturing — Service—Medical

General—overview, fiberglass and other fibers, latex, petroleum products \& lubricants, plastics and resins, solvents

No

Professional

A Overview

A.1 Occurrence of skin exposures in the workplace

A.3 Investigation, intervention, and control of occupational skin exposures

A.4 Skin physiology and function as barriers to chemical insults

B Surveillance and clinical aspects

B.1 Surveillance study reporting incidences of occupational skin exposures

B.1.A Skin exposure major focus

B.1.B Skin exposure minor focus

B.2 Loss of workdays and impact on productivity

B.3 Surveillance study protocols/procedures for gathering data

C Exposure characterization

C.1 Workplace factors associated with harmful skin exposures

F Risk management

F.1 Exposure control strategies

F.1.A Substitution

F.1.B Engineering controls

F.1.C Work practice/Administrative controls

F.1.D PPE and PPE rules

F.1.E Skin management, barrier creams, moisturizers, cleansers, and rubs

The Safety and Health Assessment and Research for Prevention (SHARP) Program at the Washington State Department of Labor and Industries conducts workrelated dermatitis research and surveillance. Under the Washington Sentinel Event Notification System for Occupational Risks (SENSOR) Dermatitis Program, SHARP has conducted surveillance on and prevention of work-related dermatitis. This Web site on skin disorders 
describes the research projects, educational materials and surveys produced by this project, as well as summaries of data collected. Examples of documents available on this Web site include:

- A guide to preventing dermatitis while working with advanced composite materials

- Metal Working Fluids: Prevention of skin problems when working with metal working fluids

- Clothing dermatitis and clothing-related skin conditions

- Skin health in agriculture

- Hand dermatitis in healthcare workers

- Prevention of hand dermatitis in the healthcare setting

- Latex sensitivity in Washington State acute care hospitals: A needs assessment and survey of awareness of the issues

- Latex sensitivity in Washington State acute care hospitals

Article ID:

221

\section{Citation:}

Resource type:

Educational materials:

Number of references:

Industries/occupations:

Specific process:

Chemical:

Specific chemicals:

Mixtures:

Audience:

Topics addressed:
Weber LW [2003]. Development of occupational skin disease. In: DiNardi SR, ed. The occupational environment: its evaluation, control, and management, 2nd ed. Fairfax, VA: AIHA.

Book/monograph, chapter

No

54

No

Professional

A Overview

A.4 Skin physiology and functions as a barrier to chemical insults

B Surveillance and clinical aspects

B.4 Clinical protocols for recognition of skin exposure health effects 


\author{
C Exposure characterization \\ C.2 Description of factors influencing exposure \\ conditions \\ C.2.A Exposure intensity/frequency/duration \\ C.2.B Exposure concentration \\ C.2.C Skin area affected \\ C.2.E Uptake \\ D Hazard identification \\ D.1 Potential health effects resulting from specific \\ chemicals \\ D.1.A Irritant contact dermatitis \\ D.1.B Allergic contact dermatitis/sensitization \\ Chapter 18, "Development of Occupational Skin Disease," \\ from the AIHA book, The Occupational Environmental: \\ Its Evaluation Control and Management (the White Book), \\ gives a brief description of skin physiology and conditions \\ that effect dermal exposure. It also discusses, for the \\ industrial hygienist, medical evaluations of the skin for \\ occupational skin disease.
}

Summary:

Article ID:

222

Citation:

Wester RC, Maibach HI [2000]. Understanding percutaneous absorption for occupational health and safety. Int J Occup Environ Health 6(2):86-92.

Resource type:

Journal article—review, meta-analysis

Educational materials:

No

Number of references:

16

Industries/occupations:

Specific process:

Chemical:

Specific chemicals:

General—overview, heavy metals/inorganic compounds, pesticides, PAHs, PCBs

DDT

Benzopyrene

Chlordane

Pentachlorophenol

PCBs

2,4 D

Arsenic

Cadmium

Mercury

Mixtures: 


\begin{tabular}{|c|c|c|}
\hline Audience: & \multicolumn{2}{|c|}{ Professional } \\
\hline \multirow[t]{4}{*}{ Topics addressed: } & $\mathrm{C}$ & Exposure characterization \\
\hline & C.2 & $\begin{array}{l}\text { Description of factors influencing exposure } \\
\text { conditions }\end{array}$ \\
\hline & C.2.E & Uptake \\
\hline & C.5 & Exposure modeling \\
\hline Summary: & \multicolumn{2}{|c|}{$\begin{array}{l}\text { This paper describes percutaneous absorption, factors } \\
\text { affecting absorption, and exposure monitoring methods. } \\
\text { It also provides percutaneous absorption rates for several } \\
\text { chemicals. }\end{array}$} \\
\hline Article ID: & \multicolumn{2}{|l|}{223} \\
\hline Citation: & \multicolumn{2}{|c|}{$\begin{array}{l}\text { Wigger-Alberti W, Elsner P [2003]. Occupational contact } \\
\text { dermatitis in the textile industry. Curr Probl Dermatol 31: } \\
114-22 \text {. }\end{array}$} \\
\hline Resource type: & \multicolumn{2}{|c|}{ Journal article-review, meta-analysis } \\
\hline Educational materials: & \multicolumn{2}{|l|}{ No } \\
\hline Number of references: & \multicolumn{2}{|l|}{68} \\
\hline Industries/occupations: & \multicolumn{2}{|c|}{ Manufacturing-Textile } \\
\hline Specific process: & \multicolumn{2}{|c|}{$\begin{array}{l}\text { Dyeing } \\
\text { Finishing }\end{array}$} \\
\hline Chemical: & \multicolumn{2}{|c|}{ Organic dyes, plastics and resins } \\
\hline Specific chemicals: & \multicolumn{2}{|c|}{ Formaldehyde } \\
\hline Mixtures: & \multicolumn{2}{|l|}{ No } \\
\hline Audience: & \multicolumn{2}{|c|}{ Professional } \\
\hline \multirow[t]{7}{*}{ Topics addressed: } & $\mathrm{B}$ & Surveillance and clinical aspects \\
\hline & B.1 & $\begin{array}{l}\text { Surveillance study reporting incidences of } \\
\text { occupational skin exposures }\end{array}$ \\
\hline & B.1.A & Skin exposure major focus \\
\hline & $\mathrm{D}$ & Hazard identification \\
\hline & D.1 & $\begin{array}{l}\text { Potential health effects resulting from specific } \\
\text { chemicals }\end{array}$ \\
\hline & D.1.A & Irritant contact dermatitis \\
\hline & D.1.B & Allergic contact dermatitis/sensitization \\
\hline Summary: & \multicolumn{2}{|c|}{$\begin{array}{l}\text { This paper discusses irritant and allergic contact } \\
\text { dermatitis in the textile industry_-primarily from resins, } \\
\text { formaldehyde, and dyes_as well as tasks with exposure } \\
\text { potential. }\end{array}$} \\
\hline
\end{tabular}


Article ID:

Citation:

Resource type:

Educational materials:

Number of references:

Industries/occupations:

Specific process:

Chemical:

Specific chemicals:

Mixtures:

Audience:

Topics addressed:

\section{4}

Winder C, Carmody M [2002]. The dermal toxicity of cement. Toxicol Ind Health 18(7):321-31.

Journal article—review, meta-analysis

No

102

Construction

Heavy metals/inorganic compounds, other: cement alkalines Chromium [III], chromium [VI] Lime (Anhydrous Calcium Hydroxide)

No

Professional

B Surveillance and clinical aspects

B.1 Surveillance study reporting incidences of occupational skin exposures

B.1.B Skin exposure minor focus

B.2 Loss of workdays and impact on productivity

D Hazard identification

D.1 Potential health effects resulting from specific chemicals

D.1.A Irritant contact dermatitis

D.1.B Allergic contact dermatitis/sensitization

D.1.D Other health effects

F Risk management

F.1 Exposure control strategies

F.1.A Substitution

F.1.C Work practice/Administrative controls

Contact dermatitis is one of the most frequently reported health problems among construction workers. Cement's alkaline ingredients (such as lime) produce irritant contact dermatitis. Ingredients, such as chromium, produce allergic contact dermatitis. This paper lists steps to reduce exposures which have been proven to reduce allergic (but not irritant) dermatitis.
Article ID:

Citation:

\section{5}

World Health Organization (WHO) [2005]. International

Programme on Chemical Safety (IPCS). [www.who.int/ ipcs/en/]. 
Resource type:

Educational materials:

Number of references:

Industries/occupations:

Specific process:

Chemical:

Specific chemicals:

Mixtures:

Audience:

Topics addressed:

Summary:
Web site

No

General—overview

General-overview, cleaning agents, coolants, corrosives, heavy metals/inorganic compounds, pesticides, petroleum products \& lubricants, plastics and resins, PAHs, PCBs, rubber additives, solvents

Variety of chemicals included in chemical-specific hazard assessments.

No

Professional

B Surveillance and clinical aspects

B.4 Clinical protocols for recognition of skin exposure health effects

D Hazard identification

D.1 Potential health effects resulting from specific chemicals

D.1.A Irritant contact dermatitis

D.1.B Allergic contact dermatitis/sensitization

D.1.C Systemic toxicity

D.1.D Other health effects

D.1.E Contribution to overall exposure

D.2 Summaries of health effects, dose-response relationships

D.3 Characterization protocols

D.3.E Measurement of skin permeation rates and reservoir effects

The IPCS is a cooperative venture between WHO, UNEP, and ILO. The two main roles of the IPCS are to establish the scientific basis for the safe use of chemicals and to strengthen national capabilities and capacities for chemical safety. A variety of resources containing information on dermal exposures and exposures to chemicals in general can be found at this Web site. Information of interest includes:

- Concise International Chemical Assessment Documents (CICADs): reviews on the effects of over 60 chemicals on human health and the environment. Over a hundred chemicals are included. The CICADs characterize the hazard and dose-response 
of exposure to chemicals and provide examples of exposure estimation and risk characterizations. Skin exposure information can be found under the occupational exposure section.

- IPCS INCHEM: access to peer-reviewed information on chemicals commonly used throughout the world and that occur as contaminants in the environment and food. IPCS INCHEM consolidates information from a number of different intergovernmental organizations.

- IPCS INTOX: a tool for poison centers and related units that provide information on preventing, evaluating, diagnosing, treating, and reporting on chemical emergencies.

- A glossary of exposure assessment-related terms: contains definitions for terms used in exposure assessment literature.

Article ID:

\section{6}

\section{Citation:}

Resource type:

Educational materials:

Number of references:

Industries/occupations:

Specific process:

Chemical:

Specific chemicals:

Mixtures:

Audience:

Topics addressed:
WHO [2005]. The IPCS: Environmental health criteria document on dermal absorption [Draft]. [www.who.int/ ipcs/methods/dermal_absorption/en/].

Technical publication/report

No

General-overview

General-overview

No

Professional

A Overview

A.4 Skin physiology and function as barriers to chemical insults

C Exposure characterization

C.2 Description of factors influencing exposure conditions

C.2.A Exposure intensity/frequency/duration

C.2.B Exposure concentration

C.2.C Skin area affected

C.2.E Uptake 
Summary:
D Hazard identification
D.3 Characterization protocols
D.3.E Measurement of skin permeation rates and reservoir effects
D.3.F QSARs—-development, validation, and application

This document provides an overview of percutaneous absorption of chemicals and the use of toxicokinetics data as part of the process of chemical risk assessment. It is not meant to be comprehensive, but rather to cover current topics of interest in the field.

Article ID:

227

Citation:

Resource type:

Educational materials:

Number of references:

Industries/occupations:

Specific process:

Chemical:

Specific chemicals:

Mixtures:

Audience:

Topics addressed:

Summary:
Wu CF, Chiu HH [2007]. Rapid method for determining dermal exposures to pesticides by use of tape stripping and FTIR spectroscopy: A pilot study. J Occup Environ Hyg 4(12):952-58.

Journal article_primary

No

29

pesticides

chloropyrifos

No

Professional

C Exposure characterization

C.4 Direct methods to measure exposure

C.4.B Skin

C.6 Other

This study ascertained the feasibility of using Fourier transform infrared spectroscopy (FTIR) to analyze tape-stripped samples to provide near real-time dermal exposure estimates. The feasibility of the stripping-FTIR approach was demonstrated.
Article ID:

Citation:

\section{8}

Zeliger HI [2003]. Toxic effects of chemical mixtures. Arch Environ Health 58(1):23-29. 
Resource type:

Educational materials:

Number of references:

Industries/occupations:

Specific process:

Chemical:

Specific chemicals:

Mixtures:

Audience:

Topics addressed:

Summary:

Article ID:

Citation:

Resource type:

Educational materials:

Number of references:

Industries/occupations:
Journal article—review, meta-analysis

No

47
Yes

Professional

C Exposure characterization

C.2 Description of factors influencing exposure conditions

C.2.E Uptake

D Hazard identification

D.3 Characterization protocols

D.3.E Measurement of skin permeation rates and reservoir effects

Exposures to chemical mixtures have reportedly produced unexpected effects. Examination of new case studies, as well as those previously reported, shows that when the human body is exposed to mixtures of chemicals that include lipophilic and hydrophilic species, the lipophiles facilitate the absorption of the hydrophiles at enhanced levels and produce effects that are not expected from individual chemicals. These effects include enhanced acute and chronic responses, low-level concentration response, and unexpected target organ attack. Octanol:water partition coefficients are predictive of relative lipophilicity and hydrophilicity. The findings have implications for safe drinking water standards, air quality standards, safe industrial and environmental exposure levels, product formulation, product labeling, and protocols for toxicity testing of chemical products.
Zhai H, Maibach HI [2004]. Dermatotoxicology, 6th ed. Boca Raton, FL: CRC Press.

Book/monograph, whole

No

3518 


\section{Specific process:}

Chemical:

Specific chemicals:

Mixtures: No

Audience:

Topics addressed:

Professional

A Overview

A.4 Skin physiology and function as barriers to chemical insults

C Exposure characterization

C.2 Description of factors influencing exposure conditions

C.2.E Uptake

C.5 Exposure modeling

D Hazard identification

D.1 Potential health effects resulting from specific chemicals

D.1.A Irritant contact dermatitis

D.1.B Allergic contact dermatitis/sensitization

D.1.C Systemic toxicity

D.1.D Other health effects

E Risk assessment

E.1 Guidelines for risk assessment or analysis

E.1.A Localized health effects

E.1.B Systemic health effects

F Risk management

F.1 Exposure control strategies

F.1.E Skin management, barrier creams, moisturizers, cleansers, and rubs

Summary:

Dermatotoxicology, 6th edition is a comprehensive reference book that includes information on the mechanisms of action of toxic substances on the skin, practical information on the various methods to evaluating dermal toxicity, and the latest developments in skin toxicology. The sixth edition contains 56 chapters, including a number of chapters covering factors influencing absorption and hazard characterization protocols, such as

1. Skin Permeability

3. Percutaneous Absorption of Complex Chemical Mixtures

4. Anatomical Factors Affecting Barrier Function 
8. Sensitive Skin

11. Irritant Dermatitis (Irritation)

12. Allergic Contact Dermatitis

13. Irritant Contact Dermatitis Versus Allergic Contact Dermatitis

14. Molecular Basis of Allergic Contact Dermatitis

15. Systemic Contact Dermatitis

16. Permeability of Human Skin to Metals and Paths for Their Diffusion

27. Barrier Creams

29. Tape Stripping Method and Stratum Corneum

36. Animal, Human, and In Vitro Test Methods for Predicting Skin Irritation

38. Test Methods for Allergic Contact Dermatitis in Animals

39. Test Methods for Allergic Contact Dermatitis in Humans

52. Evaluating Efficacy of Barrier Creams: In Vitro and In Vivo Models

53. Light-Induced Dermal Toxicity: Effects on the Cellular and Molecular Level 



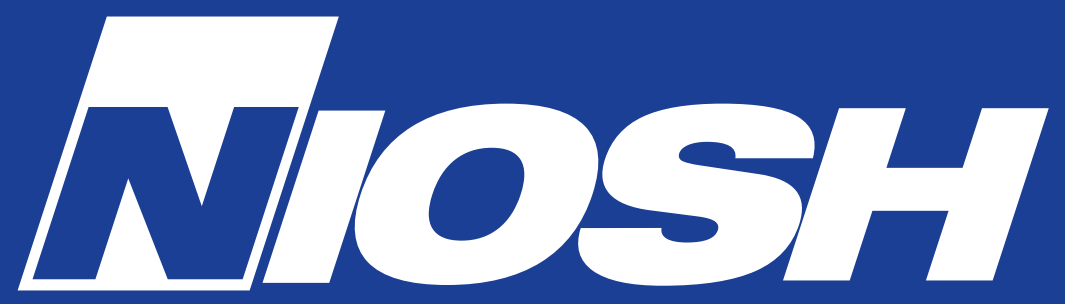

Delivering on the Nation's promise: Safety and health at work for all people through research and prevention

To receive NIOSH documents or more information about occupational safety and health topics, contact NIOSH at

1-800-CDC-INFO (1-800-232-4636)

TY: 1-888-232-6348

E-mail: cdcinfo@cdc.gov

or visit the NIOSH Web site at www.cdc.gov/niosh

For a monthly update on news at NIOSH, subscribe to NIOSH eNews by visiting www.cdc.gov/niosh/eNews.

DHHS (NIOSH) Publication No. 2009-153 